\title{
Noncommutative connections on bimodules and Drinfeld twist deformation
}

\author{
Paolo Aschieri And Alexander Schenkel
}

\begin{abstract}
Given a Hopf algebra $H$, we study modules and bimodules over an algebra $A$ that carry an $H$-action, as well as their morphisms and connections. Bimodules naturally arise when considering noncommutative analogues of tensor bundles. For quasitriangular Hopf algebras and bimodules with an extra quasi-commutativity property we induce connections on the tensor product over $A$ of two bimodules from connections on the individual bimodules. This construction applies to arbitrary connections, i.e. not necessarily $H$ equivariant ones, and further extends to the tensor algebra generated by a bimodule and its dual. Examples of these noncommutative structures arise in deformation quantization via Drinfeld twists of the commutative differential geometry of a smooth manifold, where the Hopf algebra $H$ is the universal enveloping algebra of vector fields (or a finitely generated Hopf subalgebra).

We extend the Drinfeld twist deformation theory of modules and algebras to morphisms and connections that are not necessarily $H$-equivariant. The theory canonically lifts to the tensor product structure.
\end{abstract}

1 Introduction

2 Preliminaries and notation on modules, algebras and Hopf algebras

3 Hopf algebra twists and deformations

3.1 Twist deformation preliminaries

3.2 The deformation isomorphism $D_{\mathcal{F}}$ 
4.1 Deformation of endomorphisms

4.2 Deformation of homomorphisms

4.3 Categorical formulation

5 Tensor product structure and its deformation

5.1 Triangular and quasitriangular Hopf algebras

5.2 Tensor product of $\mathbb{K}$-linear maps

5.3 Deformation

5.4 Quasi-commutative algebras and bimodules (tensor product over $A$ )

5.5 Deformation

5.6 From right to left $A$-linear homomorphisms

6 Connections

6.1 Connections on right and left modules

6.2 Deformation of connections

6.3 Connections on tensor product modules (sum of connections)

6.4 Deformation of the sum of connections

6.5 From right to left connections

6.6 Connections induced on dual modules

6.7 Connections on tensor products of modules and dual modules 


\section{Introduction}

Powerful methods for studying deformations of an algebra $A$ are available if this algebra carries a representation of a group (or Hopf algebra $H$ ). In this case one can first consider a deformation of the group in a quantum group (or of the Hopf algebra $H$ in a deformed Hopf algebra), and then use the group (or Hopf algebra) action in order to induce a deformation of the algebra $A$.

Noncommutative manifolds, i.e. noncommutative deformations of the algebra of functions on a manifold, are frequently constructed along this line, correspondingly quantum groups and Hopf algebras play a fundamental role in this field. Deformation via Drinfeld twists is an example [12, 14, 19]. Here a twist element $\mathcal{F} \in H \otimes H$ of the Hopf algebra $H$ induces a new Hopf algebra $H^{\mathcal{F}}$ and a deformed algebra $A_{\star}$. If the algebra $A$ is commutative and $H$ is co-commutative (like the universal enveloping algebra of a Lie algebra), then the commutation relations in the twist deformed algebra $A_{\star}$ are determined by the triangular $\mathcal{R}$-matrix $\mathcal{R}=\mathcal{F}_{21}^{-1} \mathcal{F}$, and $A_{\star}$ is an example of a quasicommutative algebra. The algebra $A_{\star}$ is typically noncommutative, i.e. it is a quantization of $A$.

This research area benefits from the interplay of different approaches. Let us consider for example quantum groups: They are studied as noncommutative algebras of "functions on a noncommutative group" [17], but also as quasitriangular Hopf algebras [13] together with their associated categories of representations $[14,26]$. They also originated and provided new methods in deformation quantization (see [32] for an introduction).

Noncommutative differential geometry. Hopf algebras also play a central role in the study of the differential geometry of noncommutative manifolds (the differential calculus on quantum groups [35] is a leading example).

The algebraic structures underlying noncommutative differential geometry are quite rich. If $A$ is the noncommutative analogue of the algebra of 
functions on a manifold $M$, modules over $A$ are then the noncommutative analogues of (modules of sections of) vector bundles over $M$. An analogue of the fibre-wise tensor product of vector bundles is achieved restricting to the subclass of $A$-bimodules (compatible left and right $A$-modules) and considering their tensor product over $A$. A notable example of an $A$-bimodule is that of one-forms. When the algebra $A$ carries a representation of a Hopf algebra $H$, we study ${ }_{A}{ }_{A} \mathscr{M}$-modules, which are left $H$-modules and also left $A$-modules in a compatible way. These are the noncommutative analogues of vector bundles with a lift of the $H$-action from functions on the base manifold to sections. $A$-bimodules compatible with the $H$-action, i.e. ${ }^{H}{ }_{A} \mathscr{M}_{A}$-modules, form a tensor algebra over $A$.

Noncommutative differential geometry is the study of maps between modules and bimodules, like the exterior derivative, connections and their curvatures. In particular, $A$-linear maps (left or right) are relevant because, as in the commutative case, the curvature of a connection is an $A$-linear map and also the difference between two connections is an $A$-linear map. This latter property is the affine space structure of connections.

When all modules carry an $H$-action it would seem natural to consider also $H$-equivariant maps. On the contrary, a main theme in this work is the study of the general structure of non $H$-equivariant homomorphisms, connections and curvatures. This case, for example, arises when one considers the Levi-Civita connection of a Riemannian manifold and studies deformations of the manifold that are not isometric (i.e. when the Hopf algebra $H$ is not related to the Lie algebra of Killing vector fields). More in general if the connection is a dynamical field, like in gauge and gravity theories, it is not equivariant under the $H$-action. When homomorphisms are not $H$-equivariant then they are $H$-covariant, i.e. they transform under the $H$ adjoint action. This is the canonical lift to linear and (left or right) $A$-linear maps of the $H$-action on the ${ }^{H}{ }_{A} \mathscr{M}_{A}$-modules. Thus, linear and $A$-linear maps between ${ }^{H} \mathscr{M}_{A}$-modules form also an $H$-module and their deformation can be studied via deformation of the Hopf algebra $H$.

We study the Drinfeld twist deformation theory of modules and algebras in this noncommutative differential geometric context. We develop in particular a theory of connections and of their twist deformations.

Connections in noncommutative geometry have been introduced in the mid eighties [9] and then investigated further since the mid nineties [15, $16,23,25]$. On right (or left) $A$-modules there is a well-established notion of connection, however, these connections present issues when considered on $A$-bimodules. In fact, two $A$-bimodules can be tensored into another $A$ bimodule but there is no corresponding operation on connections such that 
connections on the individual $A$-bimodules induce a connection on the tensor product $A$-bimodule. One way out is to restrict to a subclass of connections that have extra properties $[15,16,23,25]$, in particular their curvature turns out to be both left and right $A$-linear. An alternative route we advocate is to restrict to a subclass of $A$-bimodules with extra properties, so that the usual connections on right (or left) $A$-modules induce connections on tensor product modules.

Our results on the theory of connections on ${ }^{H}{ }_{A} \mathscr{M}_{A}$-modules and their twist deformation can be organized according to the extra properties we demand: i) We study the deformation of connections on right $A$-modules and the dual theory on left $A$-modules. Connections form an affine space and deformation is an affine space isomorphism. It does not preserve flatness: flat connections are deformed in non flat ones and vice versa. ii) The study of $H$-covariant (not necessarily $H$-equivariant) homomorphisms on tensor products of ${ }_{A} \mathscr{M}_{A}$-modules requires a quasitriangular Hopf algebra $H$. We first study a tensor product of linear maps compatible with the $H$ action and then show that there is a canonical way to twist deform this tensor product. iii) If furthermore the algebra $A$ and the $A$-bimodules are quasi-commutative, i.e., if they are compatible with the braiding structure of the quasitriangular Hopf algebra $H$, the tensor product of linear maps induces a tensor product over $A$ of right $A$-linear maps and we develop a theory of connections on tensor product modules. Arbitrary connections on the individual $A$-bimodules induce a connection on the tensor product $A$-bimodule. There is also a canonical extension of a connection on an $A$ bimodule to the tensor algebra generated by the $A$-bimodule and its dual. In the special case of $H$-equivariant connections we recover the usual notion of bimodule connections [15, 16, 23, 25]. An early account of our results appeared in the $\mathrm{PhD}$ thesis [29] and the proceedings articles $[1,30]$.

In the present work we have been led by the example of deformation quantization of commutative manifolds. In this case $A=C^{\infty}(M)[[h]]$ (the algebra of formal power series in $h$ with coefficients in $C^{\infty}(M)$ ) and we canonically have the Lie algebra of derivations of $A$ and the associated Hopf algebra $H=U \Xi[[h]]$, where $U \Xi$ is the universal enveloping algebra of vector fields on $M$. Vector fields and one-forms are canonically ${ }_{A}{ }_{A} \mathscr{M}_{A}$-modules, the $H$-action on these modules being via the Lie derivative. The twist deformation of these modules and of the Lie derivative and inner derivative homomorphisms has been studied in $[2,6]$ in order to formulate a noncommutative gravity theory, see [5] for a pedagogical introduction. It is deforming arbitrary connections on the tensor algebra of vector bundles over commutative manifolds that we are led to the general theory of connections on 
quasi-commutative bimodules presented in this paper. The deformation of commutative differential geometry in the more general framework of cochain twists, leading also to a nonassociative geometry, but considering only $H$ equivariant connections and homomorphisms, has been studied in [7].

In the wide class of examples obtained via twist deformation of commutative differential geometries the Hopf algebra $H$ is always triangular. A caveat is here in order: It can be that there are no nontrivial examples of truly quasitriangular Hopf algebras acting on quasi-commutative algebras and bimodules. If this is the case, then the theory of connections we present is only suitable for triangular Hopf algebras and the proofs of the theorems in this paper have the advantage of singling out the specific passages where triangularity (in the form of quasi-commutativity) is needed.

Categorical aspects of twist deformation. The categorical aspects underlying Drinfeld twist theory emerge also in the study of homomorphisms and connections. It is useful to formulate some of our findings in this language.

Let us consider the category rep $\mathrm{eq}_{\mathrm{eq}}^{H}$ of representations of the Hopf algebra $H$. The objects in rep $_{\text {eqv }}^{H}$ are $H$-modules, the morphisms are $H$-equivariant maps between $H$-modules and their composition is the usual composition $\circ$. The tensor product of representations structures rep ${ }_{\mathrm{eqv}}^{H}$ as a monoidal category, $\left(\right.$ rep eqv $\left._{\text {eq }}^{H}, \otimes\right)$. Given a twist $\mathcal{F}$ of the Hopf algebra $H$, one can deform $H$ into the Hopf algebra $H^{\mathcal{F}}$ and consider the corresponding monoidal category $\left(\right.$ rep $\left._{\text {eqv }}^{H^{\mathcal{F}}}, \otimes_{\star}\right)$ of representations of $H^{\mathcal{F}}$. The objects in rep eqv $H_{\text {eq }}^{H^{\mathcal{F}}}$ are $H^{\mathcal{F}}$ modules, the morphisms are $H^{\mathcal{F}}$-equivariant maps between $H^{\mathcal{F}}$-modules and their composition is the usual composition o. It follows from Drinfeld's work [14] that the two categories $\left(\operatorname{rep}_{\text {eqv }}^{H}, \otimes\right)$ and $\left(\operatorname{rep}_{\text {eqv }}^{H^{\mathcal{F}}}, \otimes_{\star}\right)$ are equivalent as monoidal categories. Following [14], Giaquinto and Zhang [19] studied the twist deformation of the category $\left({ }^{H}{ }_{A} \mathscr{M},{ }_{A} H o m{ }^{\text {eqv }}, \circ\right)$. In this category the objects are ${ }^{H}{ }_{A} \mathscr{M}$-modules (i.e. modules that are both left $H$-modules and left $A$-modules in a compatible way), the morphisms are $H$-equivariant and left $A$-linear maps and their composition is the usual composition o. They proved that the categories $\left({ }^{H}{ }_{A} \mathscr{M},{ }_{A} H o m^{\text {eqv }}, \circ\right)$ and $\left({ }^{H^{\mathcal{F}}}{ }_{A_{\star}} \mathscr{M}, A_{\star} H o m^{\text {eqv }}, \circ\right)$ are equivalent. 
Led by the structures required in noncommutative differential geometry we investigate the category $\left({ }^{H}{ }_{A} \mathscr{M},{ }_{A} H o m, \circ\right)$ where now morphisms are not $H$-equivariant but just left $A$-linear maps. In this case the twist does not only deform the Hopf algebra $H$, the algebra $A$ and the modules, but also the morphisms. We show that this twist deformation gives an equivalence of categories. However, this equivalence is not between the deformed category $\left({ }^{H^{\mathcal{F}}}{ }_{A_{\star}} \mathscr{M},{ }_{A_{\star}} H o m, 0\right)$ (with morphisms left $A_{\star}$-linear maps) and the initial category $\left({ }_{A}{ }_{A} \mathscr{M},{ }_{A}\right.$ Hom, o $)$, but between $\left({ }^{H^{\mathcal{F}}}{ }_{A_{\star}} \mathscr{M},{ }_{A_{\star}}\right.$ Hom, $\left.\mathrm{\circ}\right)$ and the category $\left({ }^{H}{ }_{A} \mathscr{M},{ }_{A} H o m, \circ_{\star}\right)$ obtained from $\left({ }_{A}{ }_{A} \mathscr{M},{ }_{A} H o m, \circ\right)$ by deforming just the composition law of morphisms. If we restrict to $H$-equivariant morphisms we recover the results of [19]. A similar equivalence is found when we consider the category with objects ${ }^{H} \mathscr{M}_{A}$-modules (or ${ }^{H}{ }_{A} \mathscr{M}_{A}$-modules) and morphisms right $A$-linear maps.

For quasitriangular Hopf algebras $H$ we also study the category rep ${ }^{H}$. The objects in rep ${ }^{H}$ are $H$-modules, the morphisms linear maps (not necessarily $H$-equivariant) between $H$-modules and their composition is the usual composition $\circ$. This category is an "almost monoidal" category because the tensor product on morphisms that we consider (i.e. the one compatible with the lift of the $H$-action from the tensor product of modules to the tensor product of morphisms) spoils the bifunctor properties of the tensor product (it is a bifunctor up to braiding). We show that twist deformation is however compatible with this tensor structure. Also the category with objects given by quasi-commutative ${ }_{A}{ }_{A} \mathscr{M}_{A}$-modules and morphisms given by right $A$-linear maps (and usual composition $\circ$ ) is an "almost monoidal" category. Here too we show that twist deformation is compatible with this tensor structure.

Outline. We clarify the structure of the paper by outlining its content. In Section 2 we settle our notation and we recall elementary Hopf algebra notions. In Section 3 we first introduce Hopf algebra twists and review how they induce deformations of algebras that are also $H$-modules (i.e. $H$-module algebras). Then we study algebras that transform under an $H$-adjoint action, like the algebra of linear maps of an $H$-module. The twist deformation of such an algebra leads to a deformed algebra that is isomorphic to the original one and we begin with the detailed study of this isomorphism, that we denote by $D_{\mathcal{F}}$.

Section 4 is devoted to the deformation of endomorphisms and homomorphisms between modules that carry both an $H$ and an $A$-module structure, i.e. ${ }^{H} \mathscr{M}_{A}$-modules (like e.g. the module of one-forms on a manifold). As already said, we do not restrict ourselves to $H$-equivariant maps between 
these modules. We first just consider linear maps (this is propaedeutical and will be needed for the study of connections), then we consider $A$-linear ones. Due to the isomorphism $D_{\mathcal{F}}$ between the deformed algebra of endomorphisms of a module and the algebra of endomorphisms of the deformed module, there is a canonical way to deform right $A$-linear endomorphisms

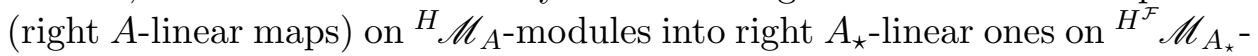
modules. Similarly, also the deformation of homomorphisms between two modules is canonical. In categorical language we have constructed a deformation functor that maps ${ }^{H} \mathscr{M}_{A^{-}}$modules to ${ }^{H^{\mathcal{F}}} \mathscr{M}_{A_{\star}}$-modules, and that has a nontrivial action, given by $D_{\mathcal{F}}$, on the corresponding right $A$-module homomorphisms. This functor implies that the category of ${ }^{H} \mathscr{M}_{A}$-modules with right $A$-linear maps as morphisms and with a twist deformed composition law is equivalent to that of $H^{\mathcal{F}} \mathscr{M}_{A_{\star}}$-modules with right $A_{\star}$-linear maps as morphisms and with the usual composition law.

In this section we also consider the deformation of ${ }^{H}{ }_{A} \mathscr{M}$-modules and of left $A$-linear homomorphisms (left $A$-linear maps). The dual $V^{\prime}$ of a right $A$ module $V$ is a left $A$-module, and we show that dualizing a deformed module is equivalent to deforming the dual module. This result will be needed later in order to study connections on dual modules and their deformation.

In Section 5 we study tensor products of modules and of homomorphisms, as well as their deformation. A tensor product of linear maps is a linear map on the tensor product of the original modules. We require $H$-covariance of this construction, i.e. that it transforms according to the $H$-adjoint action, like the original linear maps. This is achieved if the Hopf algebra is quasitriangular, in fact it is lifting the braiding of modules to linear maps (via an adjoint action) that the tensor product of linear maps is obtained. Twist deformation of homomorphisms is compatible with this tensor product, hence again there is a canonical way to deform tensor products of homomorphisms. Otherwise stated, the deformation functor is compatible with the tensor product structure. We would actually have an equivalence of monoidal categories if the tensor product between linear maps would structure the category of $H$-modules as a monoidal category. This is, however, only almost the case, since the tensor product is a bifunctor up to a braiding.

In the second part of this section we finally consider left $H$-module $A$ bimodules, i.e. ${ }^{H} \mathscr{M}_{A}$-modules. We focus on the subclass of quasi-commutative ones, i.e. of those $A$-bimodules compatible with the braiding structure of the quasitriangular Hopf algebra $H$. Correspondingly right $A$-linear homomorphisms (right $A$-linear maps) inherit a braided left $A$-linearity property. The tensor product structure we have studied before induces a tensor product structure over $A$. In particular, the tensor product of two right $A$-linear 
maps is again a right $A$-linear map on the tensor product module (over $A$ ). The deformation is also induced canonically on tensor products of quasicommutative $A$-bimodules and of their right $A$-linear homomorphisms. Otherwise stated, the deformation functor is compatible with the tensor product structure over $A$.

We conclude the section by recalling that the universal $\mathcal{R}$-matrix of a quasitriangular Hopf algebra $H$ is an example of twist of $H$. If we use it in order to twist deform quasi-commutative $A$-bimodules and their right $A$ linear homomorphisms, we obtain an isomorphism between left and right $A$-linear homomorphisms.

In Section 6 we consider a differential calculus over the algebra $A$ (a differential graded algebra over $A$ ) and connections on right $A$-modules. These are in particular linear maps between modules, and carry an affine space structure with respect to right $A$-module homomorphisms. We then deform the differential calculus and the connections. Using the results of the previous sections we obtain an affine space isomorphism between connections on right $A$-modules and deformed connections on right $A_{\star}$-modules. When the Hopf algebra is quasitriangular, and we restrict ourselves to quasi-commutative $A$ bimodules, arbitrary connections on $A$-bimodules can be summed to give a connection on the tensor product module (over $A$ ) of the initial $A$-bimodules. This operation is again compatible with twist deformation, in fact we show that the sum of deformed connections equals the deformation of the sum of connections.

Finally we study connections on dual modules. A connection on a right $A$-module induces a connection on the dual left $A$-module (and there is an affine space isomorphism between the affine spaces of connections on a right $A$-module and of connections on the dual left $A$-module). Again deformation and dualization are compatible. The dual construction can be applied to connections with right Leibniz rule on quasi-commutative $A$-bimodules to obtain connections with left Leibniz rule on the dual $A$-bimodules. These can be mapped into right Leibniz rule connections by extending the left to right isomorphism of homomorphisms that we have constructed with the $\mathcal{R}$-matrix in the end of Section 5 . In this way, from a connection on a quasicommutative $A$-bimodule we induce a connection on the tensor algebra (over $A$ ) generated by this $A$-bimodule and its dual.

In Section 7 we deform the curvature of a connection and notice that it is different from the curvature of the deformed connection. In particular, flat connections are not deformed into flat connections. Similarly we calculate the curvature of the sum of connections and find that it differs in general from the sum of the curvatures of the original connections. 


\section{Preliminaries and notation on modules, algebras and Hopf algebras}

In this section we fix the notation and recall some basic facts about Hopf algebras and their modules. In deformation quantization the field of complex numbers $\mathbb{C}$ is replaced by the ring $\mathbb{C}[[h]]$ of formal power series (in an indeterminate, say $h$ ) with coefficients in $\mathbb{C}$. In order to cover also this example we shall consider modules and algebras over a commutative ring $\mathbb{K}$ with unit element $1 \in \mathbb{K}$.

A $\mathbb{K}$-module is an abelian group $V$ together with a map $\mathbb{K} \times V \rightarrow V$, $(\lambda, v) \mapsto \lambda v$, such that for all $\lambda, \tilde{\lambda} \in \mathbb{K}$ and $v, \tilde{v} \in V$,

$$
(\tilde{\lambda} \lambda) v=\tilde{\lambda}(\lambda v), \lambda(v+\tilde{v})=\lambda v+\lambda \tilde{v},(\lambda+\tilde{\lambda}) v=\lambda v+\tilde{\lambda} v, 1 v=v .
$$

A $\mathbb{K}$-module homomorphism (or $\mathbb{K}$-linear map) between two $\mathbb{K}$-modules $V$ and $W$ is a homomorphism $P: V \rightarrow W$ of abelian groups that satisfies, for all $v \in V$ and $\lambda \in \mathbb{K}, P(\lambda v)=\lambda P(v)$. The $\mathbb{K}$-module of all $\mathbb{K}$-linear maps between $V$ and $W$ is denoted $\operatorname{Hom}_{\mathbb{K}}(V, W)$.

An algebra is a $\mathbb{K}$-module $A$ together with a $\mathbb{K}$-linear map $\mu: A \otimes A \rightarrow$ $A$ (product), where $\otimes$ is the tensor product over $\mathbb{K}$. We denote by $a \otimes b$ the image of $(a, b)$ under the canonical $\mathbb{K}$-bilinear map $A \times A \rightarrow A \otimes A$ and write for the product $\mu(a \otimes b)=a b$. The algebra $A$ is called associative if, for all $a, b, c \in A,(a b) c=a(b c)$. It is called unital if there exists a unit element $1 \in A$ satisfying $1 a=a 1=a$, for all $a \in A$. An algebra homomorphism between two algebras $A$ and $B$ is a $\mathbb{K}$-linear map $\varphi: A \rightarrow B$, such that for all $a, \tilde{a} \in A, \varphi(a \tilde{a})=\varphi(a) \varphi(\tilde{a})$. If $A$ and $B$ are unital, then $\varphi$ is also required to preserve the unit, i.e. $\varphi(1)=1$. In the following, algebras will always be associative and unital if not otherwise stated.

Definition 2.1. A Hopf algebra is an algebra $H$ together with two algebra homomorphisms $\Delta: H \rightarrow H \otimes H$ (coproduct), $\varepsilon: H \rightarrow \mathbb{K}$ (counit) and a $\mathbb{K}$-linear map $S: H \rightarrow H$ (antipode) satisfying, for all $\xi \in H$,

$$
\begin{aligned}
(\Delta \otimes \mathrm{id}) \Delta(\xi) & =(\mathrm{id} \otimes \Delta) \Delta(\xi), \quad \text { (coassociativity) } \\
(\varepsilon \otimes \mathrm{id}) \Delta(\xi) & =(\mathrm{id} \otimes \varepsilon) \Delta(\xi)=\xi \\
\mu((S \otimes \mathrm{id}) \Delta(\xi)) & =\mu((\mathrm{id} \otimes S) \Delta(\xi))=\varepsilon(\xi) 1 .
\end{aligned}
$$

The product in the algebra $H \otimes H$ is defined by, for all $\xi, \zeta, \tilde{\xi}, \tilde{\zeta} \in H$,

$$
(\xi \otimes \zeta)(\tilde{\xi} \otimes \tilde{\zeta})=(\xi \tilde{\xi}) \otimes(\zeta \tilde{\zeta})
$$


It is useful to introduce a compact notation (Sweedler's notation) for the coproduct, for all $\xi \in H, \Delta(\xi)=\xi_{1} \otimes \xi_{2}$ (sum understood). The Hopf algebra properties (2.2) in this notation read

$$
\begin{aligned}
\left(\xi_{1}\right)_{1} \otimes\left(\xi_{1}\right)_{2} \otimes \xi_{2} & =\xi_{1} \otimes\left(\xi_{2}\right)_{1} \otimes\left(\xi_{2}\right)_{2}=: \xi_{1} \otimes \xi_{2} \otimes \xi_{3} \\
\varepsilon\left(\xi_{1}\right) \xi_{2} & =\xi_{1} \varepsilon\left(\xi_{2}\right)=\xi \\
S\left(\xi_{1}\right) \xi_{2} & =\xi_{1} S\left(\xi_{2}\right)=\varepsilon(\xi) 1 .
\end{aligned}
$$

Likewise we denote the three times iterated application of the coproduct on $\xi$ by $\xi_{1} \otimes \xi_{2} \otimes \xi_{3} \otimes \xi_{4}$. It can be shown that the antipode of a Hopf algebra is unique and satisfies $S(\xi \zeta)=S(\zeta) S(\xi)$ (antimultiplicative property), $S(1)=$ $1, S\left(\xi_{1}\right) \otimes S\left(\xi_{2}\right)=S(\xi)_{2} \otimes S(\xi)_{1}$ and $\varepsilon(S(\xi))=\varepsilon(\xi)$, for all $\xi, \zeta \in H$.

Definition 2.2. Let $A$ be an algebra. A left $A$-module (or ${ }_{A} \mathscr{M}$-module) is a $\mathbb{K}$-module $V$ together with a $\mathbb{K}$-linear map $\cdot: A \otimes V \rightarrow V$ satisfying, for all $a, b \in A$ and $v \in V$,

$$
(a b) \cdot v=a \cdot(b \cdot v), 1 \cdot v=v .
$$

The map $\cdot A \otimes V \rightarrow V$ is called an action of $A$ on $V$ or a representation of $A$ on $V$.

A $\mathbb{K}$-linear map $P: V \rightarrow W$ between two ${ }_{A} \mathscr{M}$-modules $V$ and $W$ is an ${ }_{A} \mathscr{M}$-module homomorphism (or left $A$-linear map) if, for all $a \in A$ and $v \in V, P(a \cdot v)=a \cdot P(v)$. We denote the $\mathbb{K}$-module of all left $A$-linear maps between $V$ and $W$ by ${ }_{A} \operatorname{Hom}(V, W)$.

Similarly a right $A$-module (or $\mathscr{M}_{A}$-module) is a $\mathbb{K}$-module $V$ together with a $\mathbb{K}$-linear map $\cdot: V \otimes A \rightarrow V$ satisfying, for all $a, b \in A$ and $v \in V$,

$$
v \cdot(a b)=(v \cdot a) \cdot b, v \cdot 1=v .
$$

A $\mathbb{K}$-linear map $P: V \rightarrow W$ between two $\mathscr{M}_{A}$-modules $V$ and $W$ is an $\mathscr{M}_{A}$-module homomorphism (or right $A$-linear map) if, for all $a \in A$ and $v \in V, P(v \cdot a)=P(v) \cdot a$. We denote the $\mathbb{K}$-module of all right $A$-linear maps between $V$ and $W$ by $\operatorname{Hom}_{A}(V, W)$.

A left and a right module structure on $V$ are compatible if left and right actions commute. 
Definition 2.3. Let $A$ and $B$ be algebras. An $(A, B)$-bimodule (or $A_{\mathscr{M}^{-}}$ module) is a left $A$-module and a right $B$-module $V$ satisfying the compatibility condition, for all $a \in A, b \in B$ and $v \in V$,

$$
(a \cdot v) \cdot b=a \cdot(v \cdot b)
$$

In case of $B=A$ we call $V$ an $A$-bimodule (or ${ }_{A} \mathscr{M}_{A}$-module).

A $\mathbb{K}$-linear map $P: V \rightarrow W$ between two $A_{A} \mathscr{M}_{B}$-modules $V$ and $W$ is an $A \mathscr{M}_{B}$-module homomorphism if it is left $A$-linear and right $B$-linear.

The algebra $A$ can itself be a module over another algebra $H$. If $H$ is further a Hopf algebra we have the notion of an $H$-module algebra, expressing covariance of $A$ under $H$.

Definition 2.4. Let $H$ be a Hopf algebra. A left $H$-module algebra (or $H \mathscr{A}$-algebra) is an algebra $A$ which is also a left $H$-module (we denote the $H$-action by $\triangleright)$, such that for all $\xi \in H$ and $a, b \in A$,

$$
\xi \triangleright(a b)=\left(\xi_{1} \triangleright a\right)\left(\xi_{2} \triangleright b\right), \xi \triangleright 1=\varepsilon(\xi) 1
$$

An ${ }^{H} \mathscr{A}$-algebra homomorphism between two ${ }^{H} \mathscr{A}$-algebras $A$ and $B$ is an algebra homomorphism $\varphi: A \rightarrow B$ that intertwines between the left action of $H$ on $A$ and the left action of $H$ on $B$, for all $\xi \in H, a \in A, \varphi(\xi \triangleright$ $a)=\xi \triangleright \varphi(a)$. This property is also called $H$-equivariance of the algebra homomorphism $\varphi$.

We can now consider ${ }_{A} \mathscr{M}_{B}$-modules $V$, where $A, B$ are ${ }^{H} \mathscr{A}$-algebras and $V$ is also a left $H$-module. Compatibility between the Hopf algebra structure of $H$ and the $(A, B)$-bimodule structure of $V$ leads to the following covariance requirement.

Definition 2.5. Let $H$ be a Hopf algebra and $A, B$ be ${ }^{H} \mathscr{A}$-algebras. A left $H$-module $(A, B)$-bimodule (or ${ }_{A} \mathscr{M}_{B}$-module) is an ${ }_{A} \mathscr{M}_{B}$-module $V$ which is also a left $H$-module, such that for all $\xi \in H, a \in A, b \in B$ and $v \in V$,

$$
\begin{gathered}
\xi \triangleright(a \cdot v)=\left(\xi_{1} \triangleright a\right) \cdot\left(\xi_{2} \triangleright v\right), \\
\xi \triangleright(v \cdot b)=\left(\xi_{1} \triangleright v\right) \cdot\left(\xi_{2} \triangleright b\right) .
\end{gathered}
$$

In case of $B=A$ we say that $V$ is a left $H$-module $A$-bimodule (or ${ }_{A} \mathscr{M}_{A}$-module). 
An algebra $E$ is a left $H$-module $(A, B)$-bimodule algebra (or ${ }^{H} \mathscr{A}_{B^{-}}$ algebra), if $E$ as a module is an ${ }^{H}{ }_{A} \mathscr{M}_{B}$-module and if $E$ is also an ${ }^{H} \mathscr{A}$ algebra.

${ }^{H}{ }_{A} \mathscr{M}$-modules and ${ }^{H} \mathscr{M}_{B}$-modules are defined similarly to ${ }^{H}{ }_{A} \mathscr{M}_{B}$-modules, where (2.9) is restricted to (2.9a) or (2.9b), respectively. For a coherent notation we shall call left $H$-modules (where $H$ is a Hopf algebra) also ${ }^{H} \mathscr{M}$ modules.

We can consider different classes of maps between ${ }^{H}{ }_{A} \mathscr{M}_{B}$-modules. The first option is to consider maps that are compatible with all module structures, i.e. ${ }^{H}{ }_{A} \mathscr{M}_{B}$-module homomorphisms. Let $V, W$ be ${ }^{H}{ }_{A} \mathscr{M}_{B}$-modules, then a map $P: V \rightarrow W$ is an ${ }^{H}{ }_{A} \mathscr{M}_{B}$-module homomorphisms if it is an $H$ equivariant map (or ${ }^{H} \mathscr{M}$-module homomorphism), for all $\xi \in H$ and $v \in V$, $P(\xi \triangleright v)=\xi \triangleright P(v)$, and if it is also a left $A$-linear map and a right $B$-linear map. A second option, as motivated in the introduction, is to consider maps that are only compatible with the left $A$-module structure or the right $B$ module structure, i.e. left $A$-linear maps and right $B$-linear maps. A third option is to consider just $\mathbb{K}$-linear maps.

Example 2.6. Consider the universal enveloping algebra $U \Xi$ associated with the Lie algebra of vector fields $\Xi$ on a smooth manifold $M$. This is the tensor algebra (over $\mathbb{R}$ ) generated by the elements of $\Xi$ and the unit element 1 modulo the left and right ideal generated by the elements $u v-v u-[u, v]$, for all $u, v \in \Xi$. $U \Xi$ has a natural Hopf algebra structure; on the generators $u \in \Xi$ and the unit element 1 we define

$$
\begin{aligned}
\Delta(u) & =u \otimes 1+1 \otimes u, & \Delta(1) & =1 \otimes 1, \\
\varepsilon(u) & =0, & \varepsilon(1) & =1, \\
S(u) & =-u, & S(1) & =1,
\end{aligned}
$$

and extend $\Delta$ and $\varepsilon$ as algebra homomorphisms and $S$ as an antialgebra homomorphism to all $U \Xi$.

Let $V$ be the vector space of one-forms $\Omega$ (vector fields $\Xi$ ) on $M$, and $A=$ $C^{\infty}(M)$ be the algebra of smooth functions on $M . \Omega(\Xi)$ is a $C^{\infty}(M) \mathscr{M}_{C^{\infty}(M)^{-}}$ module (the right module structure equals the left module structure because $C^{\infty}(M)$ is a commutative algebra).

$C^{\infty}(M)$ is a $U \Xi \mathscr{A}$-algebra; the first of property (2.8) for $\xi$ a vector field is just the Leibniz rule. Employing the Lie derivative, we also have that $\Omega$ $(\Xi)$ is a ${ }^{U \Xi_{C}^{\infty}(M)} \mathscr{M}_{C(M)}$-module. 
Another example is obtained by considering smooth complex valued functions on $M$, and similarly vector fields and one-forms over the field $\mathbb{C}$ rather than $\mathbb{R}$.

\section{Hopf algebra twists and deformations}

We first review some well-known results on deformations of a Hopf algebra and its modules by Drinfeld twists. We then introduce a deformation isomorphism which will be of utmost importance in the study of deformations of module homomorphisms and connections.

\subsection{Twist deformation preliminaries}

Definition 3.1. Let $H$ be a Hopf algebra. A twist $\mathcal{F}$ is an element $\mathcal{F} \in$ $H \otimes H$ that is invertible and that satisfies

$$
\begin{aligned}
\mathcal{F}_{12}(\Delta \otimes \mathrm{id}) \mathcal{F} & =\mathcal{F}_{23}(\mathrm{id} \otimes \Delta) \mathcal{F}, & & (2 \text {-cocycle property }) \\
(\varepsilon \otimes \mathrm{id}) \mathcal{F} & =1=(\mathrm{id} \otimes \varepsilon) \mathcal{F}, & & \text { (normalization property })
\end{aligned}
$$

where $\mathcal{F}_{12}=\mathcal{F} \otimes 1$ and $\mathcal{F}_{23}=1 \otimes \mathcal{F}$.

We shall frequently use the notation (sum over $\alpha$ understood)

$$
\mathcal{F}=\mathrm{f}^{\alpha} \otimes \mathrm{f}_{\alpha}, \mathcal{F}^{-1}=\overline{\mathrm{f}}^{\alpha} \otimes \overline{\mathrm{f}}_{\alpha},
$$

where $\mathrm{f}^{\alpha}, \mathrm{f}_{\alpha}, \overline{\mathrm{f}}^{\alpha}, \overline{\mathrm{f}}_{\alpha}$ are elements in $H$.

In order to get familiar with this notation we rewrite (3.1a), (3.1b) and the inverse of (3.1a),

$$
\left((\Delta \otimes \mathrm{id}) \mathcal{F}^{-1}\right) \mathcal{F}_{12}^{-1}=\left((\mathrm{id} \otimes \Delta) \mathcal{F}^{-1}\right) \mathcal{F}_{23}^{-1},
$$

using the notation (3.2). Explicitly,

$$
\begin{aligned}
\mathrm{f}^{\beta} \mathrm{f}_{1}^{\alpha} \otimes \mathrm{f}_{\beta} \mathrm{f}_{2}^{\alpha} \otimes \mathrm{f}_{\alpha} & =\mathrm{f}^{\alpha} \otimes \mathrm{f}^{\beta} \mathrm{f}_{\alpha_{1}} \otimes \mathrm{f}_{\beta} \mathrm{f}_{\alpha_{2}}, \\
\varepsilon\left(\mathrm{f}^{\alpha}\right) \mathrm{f}_{\alpha} & =1=\mathrm{f}^{\alpha} \varepsilon\left(\mathrm{f}_{\alpha}\right), \\
\overline{\mathrm{f}}_{1}^{\alpha} \overline{\mathrm{f}}^{\beta} \otimes \overline{\mathrm{f}}_{2}^{\alpha} \overline{\mathrm{f}}_{\beta} \otimes \overline{\mathrm{f}}_{\alpha} & =\overline{\mathrm{f}}^{\alpha} \otimes \overline{\mathrm{f}}_{\alpha_{1}} \overline{\mathrm{f}}^{\beta} \otimes \overline{\mathrm{f}}_{\alpha_{2}} \overline{\mathrm{f}}_{\beta} .
\end{aligned}
$$

We next recall how a twist $\mathcal{F}$ induces a deformation of the Hopf algebra $H$ into a Hopf algebra $H^{\mathcal{F}}$, and of all its ${ }^{H} \mathscr{M}$-modules into $H^{\mathcal{F}} \mathscr{M}$-modules. In particular $H_{\mathscr{A}}$-algebras are deformed into $H^{\mathcal{F}} \mathscr{A}$-algebras, and commutative 
ones are typically deformed into noncommutative ones. In this respect $\mathcal{F}$ induces a quantization.

Theorem 3.2. The twist $\mathcal{F}$ of the Hopf algebra $H$ determines a new Hopf algebra $H^{\mathcal{F}}$, given by

$$
\left(H, \mu, \Delta^{\mathcal{F}}, S^{\mathcal{F}}, \varepsilon\right)
$$

As algebras $H^{\mathcal{F}}=H$ and they also have the same counit $\varepsilon^{\mathcal{F}}=\varepsilon$. The coproduct is, for all $\xi \in H$,

$$
\Delta^{\mathcal{F}}(\xi)=\mathcal{F} \Delta(\xi) \mathcal{F}^{-1}
$$

The antipode is, for all $\xi \in H$,

$$
S^{\mathcal{F}}(\xi)=\chi S(\xi) \chi^{-1}
$$

where

$$
\chi:=\mathrm{f}^{\alpha} S\left(\mathrm{f}_{\alpha}\right) \quad, \quad \chi^{-1}=S\left(\overline{\mathrm{f}}^{\alpha}\right) \overline{\mathrm{f}}_{\alpha} .
$$

A proof of this theorem can be found in textbooks on Hopf algebras, see e.g. [24], Theorem 2.3.4.

Remark 3.3. It is easy to show that the Hopf algebra $H^{\mathcal{F}}$ admits the twist $\mathcal{F}^{-1}$, indeed

$$
\mathcal{F}_{12}^{-1}\left(\Delta^{\mathcal{F}} \otimes \mathrm{id}\right) \mathcal{F}^{-1}=\mathcal{F}_{23}^{-1}\left(\mathrm{id} \otimes \Delta^{\mathcal{F}}\right) \mathcal{F}^{-1}
$$

is equivalent to (3.3). From (3.6), (3.7) and (3.8) we see that the Hopf algebra $\left(H^{\mathcal{F}}\right)^{\mathcal{F}^{-1}}$ is canonically isomorphic to $H$. We say that we twist back $H^{\mathcal{F}}$ to $H$ via the twist $\mathcal{F}^{-1}$.

Theorem 3.4. Given a Hopf algebra $H$, a twist $\mathcal{F} \in H \otimes H$ and an ${ }^{H} \mathscr{A}$ algebra $A$ (not necessarily associative or with unit), then there exists an $H^{\mathcal{F}} \mathscr{A}$-algebra $A_{\star}$. The algebra $A_{\star}$ has the same $\mathbb{K}$-module structure as $A$ and the action of $H^{\mathcal{F}}$ on $A_{\star}$ is that of $H$ on $A$. The product in $A_{\star}$ is defined by, for all $a, b \in A$,

$$
a \star b:=\mu \circ \mathcal{F}^{-1} \triangleright(a \otimes b)=\left(\overline{\mathrm{f}}^{\alpha} \triangleright a\right)\left(\overline{\mathrm{f}}_{\alpha} \triangleright b\right) .
$$

If $A$ has a unit element then $A_{\star}$ has the same unit element. If $A$ is associative then $A_{\star}$ is an associative algebra as well. 
Proof. We have to prove that the product in $A_{\star}$ is compatible with the Hopf algebra structure on $H^{\mathcal{F}}$, for all $a, b \in A$ and $\xi \in H$,

$$
\begin{aligned}
\xi \triangleright(a \star b) & =\xi \triangleright\left(\mu \circ \mathcal{F}^{-1} \triangleright(a \otimes b)\right) \\
& =\mu \circ \Delta(\xi) \triangleright \circ \mathcal{F}^{-1} \triangleright(a \otimes b) \\
& =\mu \circ\left(\Delta(\xi) \mathcal{F}^{-1}\right) \triangleright(a \otimes b) \\
& =\mu \circ \mathcal{F}^{-1} \triangleright \circ \Delta^{\mathcal{F}}(\xi) \triangleright(a \otimes b) \\
& =\left(\xi_{1_{\mathcal{F}}} \triangleright a\right) \star\left(\xi_{2_{\mathcal{F}}} \triangleright b\right),
\end{aligned}
$$

where we used the notation $\Delta^{\mathcal{F}}(\xi)=\xi_{1_{\mathcal{F}}} \otimes \xi_{2_{\mathcal{F}}}$.

If $A$ has a unit element 1 , then $1 \star a=a \star 1=a$ follows from the normalization property $(3.1 \mathrm{~b})$ of the twist $\mathcal{F}$. If $A$ is an associative algebra we also have to prove associativity of the new product, for all $a, b, c \in A$,

$$
\begin{aligned}
(a \star b) \star c & =\overline{\mathrm{f}}^{\alpha} \triangleright\left(\left(\overline{\mathrm{f}}^{\beta} \triangleright a\right)\left(\overline{\mathrm{f}}_{\beta} \triangleright b\right)\right)\left(\overline{\mathrm{f}}_{\alpha} \triangleright c\right) \\
& =\left(\overline{\mathrm{f}}^{\alpha} \triangleright a\right) \overline{\mathrm{f}}_{\alpha} \triangleright\left(\left(\overline{\mathrm{f}}^{\beta} \triangleright b\right)\left(\overline{\mathrm{f}}_{\beta} \triangleright c\right)\right)=a \star(b \star c),
\end{aligned}
$$

where we used the twist cocycle property (3.1a) in the notation adopted in $(3.4 \mathrm{c})$.

Theorem 3.5. In the hypotheses of Theorem 3.4, given another ${ }^{H} \mathscr{A}$-algebra $B$ and an ${ }^{H} \mathscr{M}_{B}$-module $V$, then there exists an ${ }^{H^{\mathcal{F}}}{ }_{A_{\star}} \mathscr{M}_{B_{\star}}$-module $V_{\star}$. The module $V_{\star}$ has the same $\mathbb{K}$-module structure as $V$ and the left action of $H^{\mathcal{F}}$ on $V_{\star}$ is that of $H$ on $V$. The $A_{\star}$ and $B_{\star}$ action on $V_{\star}$ are respectively defined by, for all $a \in A, b \in B$ and $v \in V$,

$$
\begin{aligned}
& a \star v=\cdot \circ \mathcal{F}^{-1} \triangleright(a \otimes v)=\left(\overline{\mathrm{f}}^{\alpha} \triangleright a\right) \cdot\left(\overline{\mathrm{f}}_{\alpha} \triangleright v\right), \\
& v \star b=\cdot \circ \mathcal{F}^{-1} \triangleright(v \otimes b)=\left(\overline{\mathrm{f}}^{\alpha} \triangleright v\right) \cdot\left(\overline{\mathrm{f}}_{\alpha} \triangleright b\right) .
\end{aligned}
$$

If $V=E$ is further an ${ }^{H}{ }_{A} \mathscr{A}_{B}$-algebra, then $E_{\star}$ is an ${ }^{H^{\mathcal{F}}}{ }_{A_{\star}} \mathscr{A}_{B_{\star}}$-algebra, where the product in the algebra $E_{\star}$ is given in Theorem 3.4.

Proof. We give a sketch of the proof. Left $A_{\star}$-module property:

$$
\begin{aligned}
(a \star b) \star v & =\overline{\mathrm{f}}^{\alpha} \triangleright\left(\left(\overline{\mathrm{f}}^{\beta} \triangleright a\right)\left(\overline{\mathrm{f}}_{\beta} \triangleright b\right)\right) \cdot\left(\overline{\mathrm{f}}_{\alpha} \triangleright v\right) \\
& =\left(\overline{\mathrm{f}}^{\alpha} \triangleright a\right) \cdot \overline{\mathrm{f}}_{\alpha} \triangleright\left(\left(\overline{\mathrm{f}}^{\beta} \triangleright b\right) \cdot\left(\overline{\mathrm{f}}_{\beta} \triangleright v\right)\right) \\
& =a \star(b \star v) .
\end{aligned}
$$


The right $B_{\star}$-module and $\left(A_{\star}, B_{\star}\right)$-bimodule properties are similarly proven. Compatibility between the left $H^{\mathcal{F}}$ and the left $A_{\star}$-action:

$$
\begin{aligned}
\xi \triangleright(a \star v) & =\cdot \circ\left(\Delta(\xi) \mathcal{F}^{-1}\right) \triangleright(a \otimes v)=\cdot \circ \mathcal{F}^{-1} \triangleright \circ \Delta^{\mathcal{F}}(\xi) \triangleright(a \otimes v) \\
& =\left(\xi_{1_{\mathcal{F}}} \triangleright a\right) \star\left(\xi_{2_{\mathcal{F}}} \triangleright v\right) .
\end{aligned}
$$

Compatibility between the left $H^{\mathcal{F}}$ and the right $B_{\star}$-action is similarly shown. In case $V=E$ is an ${ }^{H}{ }_{A} \mathscr{A}_{B}$-algebra, then $E_{\star}$ is an ${ }^{H^{\mathcal{F}}} A_{\star} \mathscr{A}_{B_{\star}}$-algebra because of Theorem 3.4.

As in Theorem 3.5 we can deform ${ }^{H}{ }_{A} \mathscr{M}$-modules and ${ }^{H} \mathscr{M}_{B}$-modules into $H^{\mathcal{F}}{ }_{A_{\star}} \mathscr{M}$-modules and $H^{\mathcal{F}} \mathscr{M}_{B_{\star}}$-modules by restricting (3.13) to (3.13a) or (3.13b), respectively. We can also (trivially) deform ${ }^{H} \mathscr{M}$-modules into ${ }^{H^{\mathcal{F}}} \mathscr{M}$ modules.

Example 3.6. Consider $U \mathfrak{g}[[h]]$, the universal enveloping algebra over the ring $\mathbb{C}[[h]]$ of a Lie algebra $\mathfrak{g}$. Twists $\mathcal{F} \in U \mathfrak{g} \otimes U \mathfrak{g}[[h]]$ are (up to equivalence) in one to one correspondence with skew-symmetric elements $r \in \mathfrak{g} \otimes \mathfrak{g}$ satisfying the classical Yang-Baxter equation [12].

Let $\mathcal{F}$ be a twist of (a Hopf subalgebra $U \mathfrak{g}[[h]]$ of) $U \Xi[[h]]$, the universal enveloping algebra of vector fields on a smooth manifold $M$. Then the $U \Xi[[h]] \mathscr{A}$-algebra $A=C^{\infty}(M)[[h]]$ of smooth function over $M$ with values in $\mathbb{C}[[h]]$ and the ${ }^{U \Xi[h]]} \mathscr{M}_{A}$-modules of one-forms $\Omega$ and of vector fields $\Xi$ can be deformed into the noncommutative ones $A_{\star}, \Omega_{\star}$ and $\Xi_{\star}$. Also the $U \Xi[[h]] \mathscr{A}$-algebras of tensor fields $(T, \otimes)$, of exterior forms $\left(\Omega^{\bullet}, \wedge\right)$ and the Lie algebra of vector fields $(\Xi,[]$,$) can be deformed into the noncommutative$ algebras $\left(T, \otimes_{\star}\right),\left(\Omega^{\bullet}, \wedge_{\star}\right)$ and the quantum Lie algebra $\left(\Xi,[,]_{\star}\right)$. These deformations lead to a noncommutative gravity theory [6].

Remark 3.7. The examples presented are in the context of formal deformation quantization. However, if one considers sufficiently regular actions of a group $G$, rather than actions of its Lie algebra $\mathfrak{g}$, then abelian Drinfeld twists (i.e. Drinfeld twists associated to an abelian Lie algebra $\mathfrak{g}$ ) induce $\star-$ products that can be implemented non-formally and produce deformations $A_{\star}$ of $C^{*}$-algebras [28]. This construction has further been generalized to the case of nonabelian Drinfeld twists associated to solvable Lie algebras $\mathfrak{g}$ (with extra structure) [8].

Disregarding the topological aspects, the (non-formal) noncommutative algebras of polynomial functions on multiparametric quantum groups associated with (abelian) Drinfeld twists were introduced in [27]. Their differential 
geometry and that of their homogeneous spaces was studied in [4] (and references therein).

Non-formal noncommutative geometries à la Connes related to (abelian) Drinfeld twists are, besides the noncommutative torus, the noncommutative spheres [11] and further noncommutative manifolds (so-called isospectral deformations) considered in [10, 11]. This was clarified in $[3,10,31,33]$.

\subsection{The deformation isomorphism $D_{\mathcal{F}}$}

Given an algebra $\mathbb{A}$ and an algebra homomorphism $\rho: H \rightarrow \mathbb{A}$ we can consider the adjoint action on the algebra $\mathbb{A}$, for all $\xi \in H$ and $P \in \mathbb{A}, \xi$ $P:=\rho\left(\xi_{1}\right) P \rho\left(S\left(\xi_{2}\right)\right)$. We denote adjoint actions by the symbol $>$ in order to stress that these actions are induced from "fundamental" actions (in this case the left and right actions $\rho(\xi) P$ and $P \rho(\xi)$ of the algebra $H$ on $\mathbb{A})$. In this subsection we show that twist deformation of the algebra $\mathbb{A}$ according to Theorem 3.4 and using the $H$-adjoint action $\triangleright$, gives an algebra $\mathbb{A}_{\star}$ that is isomorphic to $\mathbb{A}$. This feature has been observed for the special case of the Hopf algebra $H$ in [20] and it has been further exploited in [6] (see also [5] Section 8.2.3.1). It has been considered in the more general case of $H$-adjoint actions induced by an algebra homomorphism $\rho: H \rightarrow \mathbb{A}$ in [18] and [21].

In Theorem 3.8 and Theorem 3.9 we consider the algebra isomorphism $D_{\mathcal{F}}: \mathbb{A}_{\star} \rightarrow \mathbb{A}$ under slightly more general assumptions than the existence of an algebra homomorphism $\rho: H \rightarrow \mathbb{A}$. In Theorem 3.10 we clarify that the algebra isomorphism $D_{\mathcal{F}}: \mathbb{A}_{\star} \rightarrow \mathbb{A}$ preserves the ${ }^{H^{\mathcal{F}}} \mathscr{A}$-algebra structures of $\mathbb{A}_{\star}$ and of $\mathbb{A}$. We also show that $D_{\mathcal{F}}$ has a categorical interpretation.

Theorem 3.8. Let $\mathbb{A}$ be an ${ }^{H} \mathscr{A}$-algebra (not necessarily associative or with unit; the $H$-action is denoted by $\gg$ ) and also a right module with respect to the algebra $(H, \mu)$ (the right action of $(H, \mu)$ on $\mathbb{A}$ is simply denoted by juxtaposition), with the compatibility conditions, for all $P, Q \in \mathbb{A}$ and $\xi, \zeta \in H$,

$$
\begin{aligned}
(P Q) \xi & =P(Q \xi), \\
(P \xi) Q & =P\left(\xi_{1} \triangleright Q\right) \xi_{2}, \\
\xi \triangleright(P \zeta) & =\left(\xi_{1} \triangleright P\right)\left(\xi_{2} \triangleright \zeta\right),
\end{aligned}
$$

where $\xi \longrightarrow \zeta=\xi_{1} \zeta S\left(\xi_{2}\right)$ is the adjoint action of $H$ on $H$. In this case $\mathbb{A}$ and $\mathbb{A}_{\star}$ are isomorphic as algebras. 
Proof. As we shall explain in the text below, this theorem is equivalent to Theorem 3.9. Therefore the proof follows from the proof of Theorem 3.9.

Notice that $\mathbb{A}$ in the hypotheses above is a left module with respect to the algebra $(H, \mu)$ by defining, for all $\xi \in H$ and $P \in \mathbb{A}$,

$$
\xi P:=\left(\xi_{1} \triangleright P\right) \xi_{2} .
$$

Condition (3.16c) implies that $\mathbb{A}$ is an $(H, \mu)$-bimodule

$$
\begin{aligned}
\xi(P \zeta) & =\left(\xi_{1} \triangleright(P \zeta)\right) \xi_{2}=\left(\left(\xi_{1} \triangleright P\right)\left(\xi_{2} \triangleright \zeta\right)\right) \xi_{3} \\
& =\left(\xi_{1} \triangleright P\right) \xi_{2} \zeta S\left(\xi_{3}\right) \xi_{4}=(\xi P) \zeta .
\end{aligned}
$$

The Hopf algebra action $\longrightarrow$ on $\mathbb{A}$ is just the adjoint action with respect to this bimodule structure:

$$
\xi \triangleright P=\xi_{1} P S\left(\xi_{2}\right) .
$$

Condition (3.16b) then simply reads

$$
(P \xi) Q=P(\xi Q)
$$

and together with (3.16a) and the ${ }^{H} \mathscr{A}$-algebra property $\xi \triangleright(P Q)=\left(\xi_{1}\right.$ $P)\left(\xi_{2} \triangleright Q\right)$ we obtain

$$
\xi(P Q)=(\xi P) Q
$$

In case $\mathbb{A}$ is unital with $1 \in \mathbb{A}$ we also find, for all $\xi \in H$,

$$
\xi 1=\left(\xi_{1} \triangleright 1\right) \xi_{2}=1 \varepsilon\left(\xi_{1}\right) \xi_{2}=1 \xi
$$

Vice versa, if $\mathbb{A}$ is an algebra and an $(H, \mu)$-bimodule satisfying (3.16a), (3.20) and (3.21) (as well as (3.22) if $\mathbb{A}$ is unital), then $\xi \triangleright P:=\xi_{1} P S\left(\xi_{2}\right)$ defines an ${ }^{H} \mathscr{A}$-algebra structure on $\mathbb{A}$ that satisfies (3.16b) and (3.16c).

Hence, Theorem 3.8 equivalently reads

Theorem 3.9. Consider a Hopf algebra $H$ and an $(H, \mu)$-bimodule $\mathbb{A}$ that is also an algebra (not necessarily associative or with unit). If, for all $\xi \in H$ and $P, Q \in \mathbb{A}$, the "generalized associativity" conditions 


$$
(P Q) \xi=P(Q \xi),(P \xi) Q=P(\xi Q), \xi(P Q)=(\xi P) Q,
$$

and in case $\mathbb{A}$ is unital, with $1 \in \mathbb{A}$, also the condition

$$
\xi 1=1 \xi
$$

hold true, then the adjoint action (3.19) structures $\mathbb{A}$ as an ${ }^{H} \mathscr{A}$-algebra. Given a twist $\mathcal{F}$ of the Hopf algebra $H$, the twist deformed algebra $\mathbb{A}_{\star}$ is isomorphic (as an algebra) to $\mathbb{A}$ via the map

$$
D_{\mathcal{F}}: \mathbb{A}_{\star} \rightarrow \mathbb{A}, P \mapsto D_{\mathcal{F}}(P):=\left(\overline{\mathrm{f}}^{\alpha} \triangleright P\right) \overline{\mathrm{f}}_{\alpha}=\overline{\mathrm{f}}_{1}^{\alpha} P S\left(\overline{\mathrm{f}}_{2}^{\alpha}\right) \overline{\mathrm{f}}_{\alpha} .
$$

Proof. $D_{\mathcal{F}}$ is obviously a $\mathbb{K}$-linear map. It is also an algebra homomorphism, for all $P, Q \in \mathbb{A}$,

$$
\begin{aligned}
& D_{\mathcal{F}}(P \star Q)=D_{\mathcal{F}}\left(\left(\overline{\mathrm{f}}^{\beta} \triangleright P\right)\left(\overline{\mathrm{f}}_{\beta} \triangleright Q\right)\right) \\
& =\left(\overline{\mathrm{f}}^{\alpha} \rightarrow\left(\left(\overline{\mathrm{f}}^{\beta} \rightarrow P\right)\left(\overline{\mathrm{f}}_{\beta} \rightarrow Q\right)\right)\right) \overline{\mathrm{f}}_{\alpha} \\
& =\left(\overline{\mathrm{f}}_{1}^{\alpha} \overline{\mathrm{f}}^{\beta}-P\right)\left(\overline{\mathrm{f}}_{2}^{\alpha} \overline{\mathrm{f}}_{\beta} \triangleright Q\right) \overline{\mathrm{f}}_{\alpha} \\
& =\left(\overline{\mathrm{f}}^{\alpha} \rightarrow P\right)\left(\overline{\mathrm{f}}_{\alpha_{1}} \overline{\mathrm{f}}^{\beta} \rightarrow Q\right) \overline{\mathrm{f}}_{\alpha_{2}} \overline{\mathrm{f}}_{\beta} \\
& =\left(\overline{\mathrm{f}}^{\alpha} \triangleright P\right) \overline{\mathrm{f}}_{\alpha}\left(\overline{\mathrm{f}}^{\beta} \triangleright Q\right) \overline{\mathrm{f}}_{\beta} \\
& =D_{\mathcal{F}}(P) D_{\mathcal{F}}(Q) \text {, }
\end{aligned}
$$

where in the fourth line we used (3.4c) and in the fifth line we used that

$$
\begin{aligned}
\left(\overline{\mathrm{f}}_{\alpha_{1}} \overline{\mathrm{f}}^{\beta} \triangleright Q\right) \overline{\mathrm{f}}_{\alpha_{2}} & =\overline{\mathrm{f}}_{\alpha_{1}}\left(\overline{\mathrm{f}}^{\beta} \triangleright Q\right) S\left(\overline{\mathrm{f}}_{\alpha_{2}}\right) \overline{\mathrm{f}}_{\alpha_{3}} \\
& =\overline{\mathrm{f}}_{\alpha_{1}}\left(\overline{\mathrm{f}}^{\beta} \triangleright Q\right) \varepsilon\left(\overline{\mathrm{f}}_{\alpha_{2}}\right)=\overline{\mathrm{f}}_{\alpha}\left(\overline{\mathrm{f}}^{\beta} \triangleright Q\right) .
\end{aligned}
$$

In order to show that $D_{\mathcal{F}}$ is invertible we simplify (3.25) using (3.4) as follows

$$
\begin{aligned}
D_{\mathcal{F}}(P) & =\overline{\mathrm{f}}_{1}^{\alpha} P S\left(\overline{\mathrm{f}}_{2}^{\alpha}\right) \overline{\mathrm{f}}_{\alpha}=\overline{\mathrm{f}}^{\alpha} \mathrm{f}^{\gamma} P S\left(\overline{\mathrm{f}}_{\alpha_{1}} \overline{\mathrm{f}}^{\beta} \mathrm{f}_{\gamma}\right) \overline{\mathrm{f}}_{\alpha_{2}} \overline{\mathrm{f}}_{\beta} \\
& =\overline{\mathrm{f}}^{\alpha} \mathrm{f}^{\gamma} P S\left(\mathrm{f}_{\gamma}\right) S\left(\overline{\mathrm{f}}^{\beta}\right) \varepsilon\left(\overline{\mathrm{f}}_{\alpha}\right) \overline{\mathrm{f}}_{\beta}=\mathrm{f}^{\gamma} P S\left(\mathrm{f}_{\gamma}\right) \chi^{-1},
\end{aligned}
$$

where $\chi^{-1}=S\left(\overline{\mathrm{f}}^{\alpha}\right) \overline{\mathrm{f}}_{\alpha}$. Therefore, $D_{\mathcal{F}}$ is invertible and we have, for all $P$ $\in \mathbb{A}$,

$$
D_{\mathcal{F}}{ }^{-1}(P)=\overline{\mathrm{f}}^{\alpha} P \chi S\left(\overline{\mathrm{f}}_{\alpha}\right)
$$

where $\chi=\mathrm{f}^{\beta} S\left(\mathrm{f}_{\beta}\right)$. 
Finally, if $\mathbb{A}$ is unital, $D_{\mathcal{F}}$ maps the unit of $\mathbb{A}_{\star}$ to the unit of $\mathbb{A}$ because of the normalization property of the twist $(\varepsilon \otimes \mathrm{id}) \mathcal{F}^{-1}=1$.

We introduce the triple notation $(\mathbb{A}, \mu, \nabla)$ in order to explicitly write the product and the $H$-action of an ${ }^{H} \mathscr{A}$-algebra $\mathbb{A}$. Then the ${ }^{H^{\mathcal{F}}} \mathscr{A}$-algebra $\mathbb{A}_{\star}$ is described by the triple $\left(\mathbb{A}, \mu_{\star}, \nabla\right)$.

In the hypotheses of Theorem 3.9, the Hopf algebra property (2.4c) immediately implies that the algebra $\mathbb{A}$ has an $H^{\mathcal{F}} \mathscr{A}$-algebra structure given by the $H^{\mathcal{F}}$-adjoint action, for all $\xi \in H^{\mathcal{F}}$ and $P \in \mathbb{A}$,

$$
\xi \triangleright_{\mathcal{F}} P:=\xi_{1_{\mathcal{F}}} P S^{\mathcal{F}}\left(\xi_{2_{\mathcal{F}}}\right) \text {. }
$$

We denote this $H^{\mathcal{F}} \mathscr{A}$-algebra by $\left(\mathbb{A}, \mu, \boldsymbol{F}_{\mathcal{F}}\right)$. We have the following

Theorem 3.10. The algebra isomorphism $D_{\mathcal{F}}: \mathbb{A}_{\star} \rightarrow \mathbb{A}$ of Theorem 3.9 is also an isomorphism between the ${ }^{H^{\mathcal{F}}} \mathscr{A}$-algebras $\left(\mathbb{A}, \mu_{\star}, \boldsymbol{}\right)$ and $\left(\mathbb{A}, \mu, \boldsymbol{F}_{\mathcal{F}}\right)$, i.e. $D_{\mathcal{F}}$ intertwines between the $H^{\mathcal{F}}$-actions $\$ and $\boldsymbol{\mathcal { F }}_{\mathcal{F}}$, for all $\xi \in H^{\mathcal{F}}$ and $P \in \mathbb{A}$,

$$
D_{\mathcal{F}}(\xi \triangleright P)=\xi \triangleright_{\mathcal{F}} D_{\mathcal{F}}(P) .
$$

Proof. Using (3.28) we obtain, for all $\xi \in H^{\mathcal{F}}$ and $P \in \mathbb{A}_{\star}$,

$$
\begin{aligned}
D_{\mathcal{F}}(\xi \triangleright P) & =\mathrm{f}^{\beta}(\xi \triangleright P) S\left(\mathrm{f}_{\beta}\right) \chi^{-1}=\mathrm{f}^{\beta} \xi_{1} P S\left(\xi_{2}\right) S\left(\mathrm{f}_{\beta}\right) \chi^{-1} \\
& =\mathrm{f}^{\beta} \xi_{1} \overline{\mathrm{f}}^{\gamma} \mathrm{f}^{\delta} P S\left(\mathrm{f}_{\beta} \xi_{2} \overline{\mathrm{f}}_{\gamma} \mathrm{f}_{\delta}\right) \chi^{-1} \\
& =\xi_{1_{\mathcal{F}}} \mathrm{f}^{\delta} P S\left(\mathrm{f}_{\delta}\right) \chi^{-1} \chi S\left(\xi_{2_{\mathcal{F}}}\right) \chi^{-1} \\
& =\xi_{1_{\mathcal{F}}} D_{\mathcal{F}}(P) S^{\mathcal{F}}\left(\xi_{2_{\mathcal{F}}}\right) \\
& =\xi \triangleright D_{\mathcal{F}} D_{\mathcal{F}}(P)
\end{aligned}
$$

where in the third equality we inserted $1 \otimes 1=\mathcal{F}^{-1} \mathcal{F}$.

Remark 3.11. We have discussed in Remark 3.3 that $H^{\mathcal{F}}$ admits the twist $\mathcal{F}^{-1}$ leading to $\left(H^{\mathcal{F}}\right)^{\mathcal{F}^{-1}}=H$. The associated deformation isomorphism $D_{\mathcal{F}^{-1}}$ is exactly $D_{\mathcal{F}^{-1}}$ given in (3.29). This can be shown by using (3.28) and a short calculation, for all $P \in \mathbb{A}$,

$$
\begin{aligned}
D_{\mathcal{F}^{-1}}(P) & =\left(\mathrm{f}^{\beta} \boldsymbol{\mathcal { F }}_{\mathcal{F}} P\right) \mathrm{f}_{\beta}=\overline{\mathrm{f}}^{\beta} P S^{\mathcal{F}}\left(\overline{\mathrm{f}}_{\beta}\right) \chi_{\mathcal{F}}^{-1} \\
& =\overline{\mathrm{f}}^{\beta} P \chi S\left(\overline{\mathrm{f}}_{\beta}\right) \chi^{-1} \chi=D_{\mathcal{F}}{ }^{-1}(P),
\end{aligned}
$$

where we also have used that $\chi_{\mathcal{F}}^{-1}=S^{\mathcal{F}}\left(\mathrm{f}^{\beta}\right) \mathrm{f}_{\beta}=\chi$. 
Example 3.12. Given an ${ }^{H} \mathscr{A}$-algebra $A$ (not necessarily associative or with unit) we consider the crossed product (or smash product) algebra $A \rtimes H$. By definition the underlying $\mathbb{K}$-module structure is $A \otimes H$ and the product is given by $(a \otimes \xi)(b \otimes \zeta)=a\left(\xi_{1} \triangleright b\right) \otimes \xi_{2} \zeta$, that we simply rewrite as

$$
a \xi b \zeta=a\left(\xi_{1} \triangleright b\right) \xi_{2} \zeta
$$

The algebra $A \rtimes H$ is an ${ }^{H} \mathscr{A}$-algebra with the action $\xi \triangleright(a \zeta):=\left(\xi_{1} \triangleright a\right)\left(\xi_{2}\right.$ - $\zeta)$. The right $(H, \mu)$-module structure is given by $(a \xi) \zeta=a(\xi \zeta)$ and the compatibility conditions (3.16) hold true. Hence the hypotheses of Theorem 3.8 are satisfied.

Corollary 3.13. Deformation of an ${ }^{H} \mathscr{A}$-algebra $A$ is the restriction to $A$ of the deformed algebra $(A \rtimes H)_{\star}$ that is isomorphic to $A \rtimes H$ because of Theorem 3.8.

If $M$ is a smooth manifold, $A=C^{\infty}(M)$ and $H=U \Xi$ is the universal enveloping algebra of vector fields on $M$, then $A \rtimes H$ is the algebra of differential operators. Up to considering the extension of these algebras to the ring $\mathbb{C}[[h]]$, we have that the map $D_{\mathcal{F}}: A \rightarrow D_{\mathcal{F}}(A) \subset A \rtimes U \Xi$ realizes a quantization of $A$ in terms of differential operators $[2,34]$.

Example 3.14. Given an algebra $\mathbb{A}$ that admits an algebra homomorphism $\rho: H \rightarrow \mathbb{A}$, then the hypotheses of Theorem 3.9 immediately hold. Just define the $(H, \mu)$-bimodule structure of $\mathbb{A}$ by, for all $\xi \in H, P \in \mathbb{A}$, $\xi P:=\rho(\xi) P$ and $P \xi:=P \rho(\xi)$. A particular case is when $\mathbb{A}=H$ and we consider the identity homomorphism. Then we recover the (Hopf algebra) isomorphism $D: H_{\star} \rightarrow H^{\mathcal{F}}$ discussed in [6].

Example 3.15. Given a Hopf algebra $H$ and an ${ }^{H} \mathscr{M}$-module $V$ we consider the algebra $\operatorname{End}_{\mathbb{K}}(V)$ of $\mathbb{K}$-linear maps ( $\mathbb{K}$-module homomorphisms) from $V$ to $V$. Since $H$ is a Hopf algebra the left action of $H$ on $V$ lifts to a left action of $H$ on $\operatorname{End}_{\mathbb{K}}(V)$, defined by, for all $P \in \operatorname{End}_{\mathbb{K}}(V)$ and $\xi \in H$,

$$
\xi \triangleright P:=\xi_{1} \triangleright \circ P \circ S\left(\xi_{2}\right) \triangleright
$$

where $\circ$ denotes the usual composition of morphisms and $\xi \triangleright \in \operatorname{End}_{\mathbb{K}}(V)$ is the endomorphism $v \mapsto \xi \triangleright v$. The algebra $\operatorname{End}_{\mathbb{K}}(V)$ is thus an ${ }^{H} \mathscr{A}$-algebra, and we denote it also by the triple $\left(\operatorname{End}_{\mathbb{K}}(V), \circ, \downarrow\right)$ in order to explicitly refer to the product and the $H$-action. The algebra homomorphism $H \rightarrow \operatorname{End}_{\mathbb{K}}(V), \xi \mapsto \xi \triangleright$ implies (see Example 3.14) the isomorphism $D_{\mathcal{F}}$ : 
$\operatorname{End}_{\mathbb{K}}(V)_{\star} \rightarrow \operatorname{End}_{\mathbb{K}}(V)$. The composition law in $\operatorname{End}_{\mathbb{K}}(V)_{\star}$ is given by the $\star$-composition $P \circ_{\star} Q:=\left(\overline{\mathrm{f}}^{\alpha} \triangleright P\right) \circ\left(\overline{\mathrm{f}}_{\alpha} \triangleright Q\right)$, for all $P, Q \in \operatorname{End}_{\mathbb{K}}(V)_{\star}$. Because of Theorem 3.10 we further obtain that $D_{\mathcal{F}}$ is an ${ }^{H^{\mathcal{F}}} \mathscr{A}$-algebra isomorphism between $\left(\operatorname{End}_{\mathbb{K}}(V), \circ_{\star}, \boldsymbol{\nabla}\right)$ and $\left(\operatorname{End}_{\mathbb{K}}(V), \circ, \boldsymbol{\mathcal { F }}_{\mathcal{F}}\right)$.

Notice that since as algebras $H^{\mathcal{F}}=H$, the deformed ${ }^{H^{\mathcal{F}}} \mathscr{\mathscr { M }}$-module $V_{\star}$ of Theorem 3.5 (with trivial algebras $A=B=\mathbb{K}$ ) is equal to the ${ }^{H} \mathscr{M}$-module $V$ and henceforth we can identify the ${ }^{H^{\mathcal{F}}} \mathscr{A}$-algebras $\left(\operatorname{End}_{\mathbb{K}}(V), \circ, \boldsymbol{}_{\mathcal{F}}\right)$ and $\left(\operatorname{End}_{\mathbb{K}}\left(V_{\star}\right), \circ, \boldsymbol{\nabla}_{\mathcal{F}}\right)$. Thus, $D_{\mathcal{F}}$ is also an isomorphism between the $H^{\mathcal{F}} \mathscr{A}$ algebras $\left(\operatorname{End}_{\mathbb{K}}(V), \circ_{\star}, \triangleright\right)$ and $\left(\operatorname{End}_{\mathbb{K}}\left(V_{\star}\right), \circ, \boldsymbol{\mathcal { F }}_{\mathcal{F}}\right)$.

Categorical point of view. We provide a generalization of Example 3.15. Instead of studying a fixed ${ }^{H} \mathscr{M}$-module $V$, let us consider the category rep $^{H}$ of representations of $H$. An object in rep ${ }^{H}$ is an ${ }^{H} \mathscr{M}$-module $V$ and a morphism between two objects $V, W$ in $\operatorname{rep}^{H}$ is a $\mathbb{K}$-linear map $P: V \rightarrow W$, i.e. $P \in \operatorname{Hom}_{\mathbb{K}}(V, W)$. Notice that we do not assume the map $P$ to be $H$ equivariant. The composition of morphisms is the usual composition $\circ$. Given a twist $\mathcal{F} \in H \otimes H$ of the Hopf algebra $H$ we can consider the category rep $_{\star}^{H}$. The objects and morphisms in rep $_{\star}{ }_{\star}$ are the same as the objects and morphisms in rep $^{H}$, but the composition is given by the $\star$-composition ${ }_{\star}$, where the $H$-action on morphisms is given by the $H$-adjoint action (cf. (3.35)), canonically obtained lifting the source and target $H$-actions.

Theorem 3.16. Let $H$ be a Hopf algebra with twist $\mathcal{F} \in H \otimes H$. Then there is a functor from $\mathrm{rep}^{H}{ }$ to rep $^{H}$ that is the identity on objects, and associates to any morphism $P: V \rightarrow W$ the morphism

$$
\begin{aligned}
D_{\mathcal{F}}(P): V & \longrightarrow W, \\
v & \longmapsto\left(\overline{\mathrm{f}}^{\alpha} \triangleright P\right)\left(\overline{\mathrm{f}}_{\alpha} \triangleright v\right)=\overline{\mathrm{f}}_{1}^{\alpha} \triangleright\left(P\left(S\left(\overline{\mathrm{f}}_{2}^{\alpha}\right) \overline{\mathrm{f}}_{\alpha} \triangleright v\right)\right) .
\end{aligned}
$$

Furthermore, the categories rep $^{H} \star$ and rep $^{H}$ are equivalent.

Proof. $D_{\mathcal{F}}(P)=\left(\overline{\mathrm{f}}^{\alpha} \triangleright P\right) \circ \overline{\mathrm{f}}_{\alpha} \triangleright$ is obviously a $\mathbb{K}$-linear map. We also have that $D_{\mathcal{F}}\left(\mathrm{id}_{V}\right)=\left(\overline{\mathrm{f}}^{\alpha} \nabla \mathrm{id}_{V}\right) \circ \overline{\mathrm{f}}_{\alpha} \triangleright=\mathrm{id}_{V}$, because of the normalization condition $(3.1 \mathrm{~b})$ of the twist. In order to show that $D_{\mathcal{F}}$ preserves composition of morphisms, i.e. that for any two composable morphisms $P$ and $Q$ in $\operatorname{rep}^{H}$ * we have $D_{\mathcal{F}}\left(P \circ_{\star} Q\right)=D_{\mathcal{F}}(P) \circ D_{\mathcal{F}}(Q)$, we repeat the same passages as in the proof of Theorem 3.9.

This functor has a left and right inverse given by the functor that is the identity on objects and that to any morphism $P: V \rightarrow W$ in rep $^{H}$ associates the morphism $D_{\mathcal{F}^{-1}}(P)=D_{\mathcal{F}}^{-1}(P): V \rightarrow W$ in rep ${ }^{H} \star$ (cf. Remark 3.11). Hence the two categories are equivalent. 
In the end of Example 3.15 we have identified the ${ }^{H^{\mathcal{F}}} \mathscr{A}$-algebras $\left(\operatorname{End}_{\mathbb{K}}(V)\right.$, $\left.\circ, \triangleright_{\mathcal{F}}\right)$ and $\left(\operatorname{End}_{\mathbb{K}}\left(V_{\star}\right), \circ, \boldsymbol{F}_{\mathcal{F}}\right)$. Here we can similarly identify the category rep $^{H}$ with the category rep ${ }^{H^{\mathcal{F}}}$ of ${ }^{H^{\mathcal{F}}} \mathscr{M}$-modules with $\mathbb{K}$-linear maps as morphisms. Indeed any ${ }^{H} \mathscr{M}$-module $V$ can be seen as an ${ }^{H^{\mathcal{F}}} \mathscr{M}$-module (denoted $\left.V_{\star}\right)$. It follows that $D_{\mathcal{F}}$ provides an equivalence between the categories rep ${ }^{H} \star$ and $\operatorname{rep}^{H^{\mathcal{F}}}$. In particular, any morphism $P: V \rightarrow W$ in rep ${ }^{H} \star$ is mapped to a morphism $D_{\mathcal{F}}(P): V_{\star} \rightarrow W_{\star}$ in $\operatorname{rep}^{H^{\mathcal{F}}}$.

\section{Module homomorphisms}

Let $A, B$ be two ${ }^{H} \mathscr{A}$-algebras and $V, W$ be two ${ }^{H}{ }_{A} \mathscr{M}_{B}$-modules. In this section we study the properties of $\mathbb{K}$-linear maps $\operatorname{Hom}_{\mathbb{K}}(V, W)$ and right $B$-linear maps $\operatorname{Hom}_{B}(V, W)$, and their deformations. The $H$-action on the modules $V$ and $W$ lifts to an $H$-adjoint action on these maps; in general this adjoint action is nontrivial because the maps are not $H$-equivariant. To any map $P: V \rightarrow W$ we associate a deformed map $D_{\mathcal{F}}(P): V_{\star} \rightarrow W_{\star}$, where the deformed ${ }_{A_{\star}}^{H^{\mathcal{F}}} \mathscr{M}_{B_{\star}}$-modules $V_{\star}$ and $W_{\star}$ are obtained according to Theorem 3.5. We show that this correspondence is a bijection between $\mathbb{K}$-linear maps (i.e. between $\operatorname{Hom}_{\mathbb{K}}(V, W)$ and $\operatorname{Hom}_{\mathbb{K}}\left(V_{\star}, W_{\star}\right)$ ), and also between right $B$-linear and right $B_{\star}$-linear maps (i.e. between $\operatorname{Hom}_{B}(V, W)$ and $\operatorname{Hom}_{B_{\star}}\left(V_{\star}\right.$, $\left.\left.W_{\star}\right)\right)$.

We further clarify the algebraic structures preserved by $D_{\mathcal{F}}$. In particular we extend the result obtained in Example 3.15 where $D_{\mathcal{F}}$ was shown to be an isomorphism between the ${ }^{H^{\mathcal{F}}} \mathscr{A}$-algebras $\left(\operatorname{End}_{\mathbb{K}}(V), \circ_{\star}, \boldsymbol{\bullet}\right)$ and $\left(\operatorname{End}_{\mathbb{K}}\left(V_{\star}\right)\right.$, $\left.\circ, \nabla_{\mathcal{F}}\right)$.

Finally, for later use, we consider a mirror construction that deforms left $A$-linear maps of ${ }^{H}{ }_{A} \mathscr{M}_{B}$-modules into left $A_{\star}$-linear maps of ${ }^{H^{\mathcal{F}}}{ }_{A_{\star}} \mathscr{M}_{B_{\star}}$ modules. We conclude with a categorical description of the results obtained.

\subsection{Deformation of endomorphisms}

In this subsection we study the algebras $\operatorname{End}_{\mathbb{K}}(V)$ and $\operatorname{End}_{B}(V)$ of endomorphisms of an ${ }^{H}{ }_{A} \mathscr{M}_{B}$-module $V$, where $A$ and $B$ are ${ }^{H} \mathscr{A}$-algebras. In particular we focus on the canonical $A$-bimodule structure of $\operatorname{End}_{\mathbb{K}}(V)$ and $\operatorname{End}_{B}(V)$ induced by the ${ }^{H} \mathscr{A}$-algebra homomorphism $l: A \rightarrow \operatorname{End}_{\mathbb{K}}(V)$ (see Proposition 4.1). This structure will also be relevant in the next subsection, where we discuss homomorphisms between different ${ }^{H}{ }_{A} \mathscr{M}_{B}$-modules $V, W$.

The behavior of the endomorphism algebras $\operatorname{End}_{\mathbb{K}}(V)$ and $\operatorname{End}_{B}(V)$ under twist deformation is studied. There are two equivalent deformations 
of $\operatorname{End}_{B}(V)$ : The $\operatorname{End}_{B}(V)_{\star}$ deformation is obtained by considering endomorphisms as elements of the algebra $\operatorname{End}_{B}(V)$. The $\operatorname{End}_{B_{\star}}\left(V_{\star}\right)$ deformation is obtained by considering them as right $B$-linear maps on $V$ and deforming the module $V$. The equivalence of the two deformations is provided by the map $D_{\mathcal{F}}$ from right $B$-linear to right $B_{\star}$-linear endomorphisms.

Proposition 4.1. Let $A$ be an ${ }^{H} \mathscr{A}$-algebra and $V$ be an ${ }^{{ }^{H}}{ }_{A} \mathscr{M}$-module. Then the algebra $\operatorname{End}_{\mathbb{K}}(V)$ of $\mathbb{K}$-linear maps from $V$ to $V$ is an ${ }^{H}{ }_{A} \mathscr{A}_{A}$-algebra with the $H$-adjoint action, for all $\xi \in H$ and $P \in \operatorname{End}_{\mathbb{K}}(V)$,

$$
\xi \triangleright P:=\xi_{1} \triangleright \circ P \circ S\left(\xi_{2}\right) \triangleright
$$

and the A-bimodule structure given by, for all $a \in A$ and $P \in \operatorname{End}_{\mathbb{K}}(V)$,

$$
\begin{aligned}
& a \cdot P:=l_{a} \circ P, \\
& P \cdot a:=P \circ l_{a},
\end{aligned}
$$

where, for all $v \in V, l_{a}(v):=a \cdot v$.

If $B$ is another ${ }^{H} \mathscr{A}$-algebra and $V$ is also an ${ }^{H}{ }_{A} \mathscr{M}_{B}$-module, then the subalgebra $\operatorname{End}_{B}(V) \subset \operatorname{End}_{\mathbb{K}}(V)$ of right $B$-linear endomorphisms of $V$, (i.e. $P \in \operatorname{End}_{B}(V)$ if, for all $v \in V$ and $\left.b \in B, P(v \cdot b)=P(v) \cdot b\right)$ is an ${ }_{A} \mathscr{A}_{A^{-}}$ subalgebra with $H$ and $A$ actions given in (4.1) and (4.2), respectively.

Proof. $\operatorname{End}_{\mathbb{K}}(V)$ is a left $A$-module, for all $a, \tilde{a} \in A$ and $P \in \operatorname{End}_{\mathbb{K}}(V)$,

$$
a \cdot(\tilde{a} \cdot P)=l_{a} \circ l_{\tilde{a}} \circ P=l_{a \tilde{a}} \circ P=(a \tilde{a}) \cdot P .
$$

Similarly, we have that $\operatorname{End}_{\mathbb{K}}(V)$ is a right $A$-module. It is a bimodule because $\left(l_{a} \circ P\right) \circ l_{\tilde{a}}=l_{a} \circ\left(P \circ l_{\tilde{a}}\right)$.

It is straightforward to check that $\operatorname{End}_{\mathbb{K}}(V)$ is an ${ }^{H} \mathscr{A}$-algebra, for all $\xi, \zeta \in H$ and $P, Q \in \operatorname{End}_{\mathbb{K}}(V)$,

$$
\xi \triangleright(\zeta>P)=(\xi \zeta)>P, \quad \xi \triangleright \mathrm{id}_{V}=\varepsilon(\xi) \mathrm{id}_{V}
$$

and

$$
\xi \triangleright(P \circ Q)=\left(\xi_{1} \triangleright P\right) \circ\left(\xi_{2} \triangleright Q\right)
$$

We now prove that the algebra homomorphism $l: A \rightarrow \operatorname{End}_{\mathbb{K}}(V)$ given by $a \mapsto l_{a}$ is also an ${ }^{H} \mathscr{A}$-algebra homomorphism, i.e. for all $a \in A$ and $\xi \in H$, 
$l_{\xi \triangleright a}=\xi \triangleright l_{a}$. Indeed, for all $v \in V$,

$$
\begin{aligned}
\left(\xi \triangleright l_{a}\right)(v) & =\xi_{1} \triangleright\left(l_{a}\left(S\left(\xi_{2}\right) \triangleright v\right)\right)=\xi_{1} \triangleright\left(a \cdot\left(S\left(\xi_{2}\right) \triangleright v\right)\right) \\
& =\xi_{1} \triangleright a \cdot\left(\xi_{2} S\left(\xi_{3}\right) \triangleright v\right)=(\xi \triangleright a) \cdot v=l_{\xi \triangleright a}(v) .
\end{aligned}
$$

Compatibility between the $H$-module structure and the $A$-bimodule structure, i.e. for all $\xi \in H, a \in A$ and $P \in \operatorname{End}_{\mathbb{K}}(V), \xi \triangleright(a \cdot P)=\left(\xi_{1} \triangleright a\right) \cdot\left(\xi_{2}\right.$ $P)$ and $\xi \triangleright(P \cdot a)=\left(\xi_{1} \triangleright P\right) \cdot\left(\xi_{2} \triangleright a\right)$, follows from (4.4b) and (4.5).

Finally let $V$ be an ${ }^{H}{ }_{A} \mathscr{M}_{B}$-module, then $V$ is in particular an $(A, B)$ bimodule (i.e. for all $a \in A, b \in B$ and $v \in V$, we have $a \cdot(v \cdot b)=(a \cdot v) \cdot b$ ), hence $l_{a} \in \operatorname{End}_{B}(V)$. Therefore, $a \cdot P \in \operatorname{End}_{B}(V)$ and $P \cdot a \in \operatorname{End}_{B}(V)$ if $P \in \operatorname{End}_{B}(V)$. Furthermore, for all $\xi \in H$ and $P \in \operatorname{End}_{B}(V)$ we have $\xi$ $P \in \operatorname{End}_{B}(V)$, indeed, for all $b \in B$ and $v \in V$,

$$
\begin{aligned}
(\xi \triangleright P)(v \cdot b) & =\xi_{1} \triangleright\left(P\left(\left(S\left(\xi_{3}\right) \triangleright v\right) \cdot\left(S\left(\xi_{2}\right) \triangleright b\right)\right)\right) \\
& =\left(\xi_{1} \triangleright\left(P\left(S\left(\xi_{4}\right) \triangleright v\right)\right)\right) \cdot\left(\xi_{2} \triangleright\left(S\left(\xi_{3}\right) \triangleright b\right)\right) \\
& =(\xi \triangleright P)(v) \cdot b .
\end{aligned}
$$

Let $H$ be a Hopf algebra with twist $\mathcal{F} \in H \otimes H$. Let also $A, B$ be two ${ }^{H} \mathscr{A}$-algebras and $V$ an ${ }^{H} \mathscr{M}_{B}$-module, so that $\operatorname{End}_{\mathbb{K}}(V)$ and $\operatorname{End}_{B}(V)$ are $H_{A} \mathscr{A}_{A}$-algebras. In order to explicitly write the product, module structure and $H$-action of these ${ }^{H} \mathscr{A}_{A}$-algebras we use the quadruples $\left(\operatorname{End}_{\mathbb{K}}(V), \circ, \cdot\right.$, -) and $\left(\operatorname{End}_{B}(V), \circ, \cdot, \mathbf{}\right)$.

We have two deformations of the endomorphisms $\operatorname{End}_{\mathbb{K}}(V), \operatorname{End}_{B}(V)$ : The first option, $\operatorname{End}_{\mathbb{K}}(V)_{\star}$ and $\operatorname{End}_{B}(V)_{\star}$, is to consider the ${ }^{H^{\mathcal{F}}}{ }_{A_{\star}} \mathscr{A}_{A_{\star}}$ algebras obtained by applying Theorem 3.5 to $\operatorname{End}_{\mathbb{K}}(V)$ and $\operatorname{End}_{B}(V)$. It is characterized by a deformed composition law and a deformed module structure, for all $P, Q \in \operatorname{End}_{\mathbb{K}}(V)_{\star}$ and $a \in A_{\star}$,

$$
P \circ_{\star} Q:=\left(\overline{\mathrm{f}}^{\alpha} \triangleright P\right) \circ\left(\overline{\mathrm{f}}_{\alpha} \triangleright Q\right), a \star P:=l_{a} \circ_{\star} P, P \star a:=P \circ_{\star} l_{a} .
$$

In quadruple notation, these ${ }^{H^{\mathcal{F}}} A_{\star} \mathscr{A}_{A_{\star}}$-algebras are denoted by $\left(\operatorname{End}_{\mathbb{K}}(V)\right.$, $\left.\circ_{\star}, \star, \triangleright\right)$ and $\left(\operatorname{End}_{B}(V), \circ_{\star}, \star, \downarrow\right)$. The second option is to consider the $\mathbb{K}$ linear or right $B_{\star}$-linear endomorphisms of the deformed ${ }^{H^{\mathcal{F}}}{ }_{A_{\star}} \mathscr{M}_{B_{\star}}$-module $V_{\star}$. From Proposition 4.1 we know that $\operatorname{End}_{\mathbb{K}}\left(V_{\star}\right)$ and $\operatorname{End}_{B_{\star}}\left(V_{\star}\right)$ are $H_{A_{\star}}^{H^{\mathcal{F}}} \mathscr{A}_{A_{\star}}$-algebras, where the product is the usual composition. The $A_{\star}-$ bimodule structure is induced by the ${ }^{H^{\mathcal{F}}} \mathscr{A}$-algebra homomorphism $l^{\star}: A_{\star} \rightarrow$ 
$\operatorname{End}_{\mathbb{K}}\left(V_{\star}\right)$ given by, for all $a \in A_{\star}$ and all $v \in V_{\star}, l_{a}^{\star}(v):=a \star v$. It explicitly reads, for all $a \in A_{\star}$ and $P_{\star} \in \operatorname{End}_{\mathbb{K}}\left(V_{\star}\right)$,

$$
a \cdot P_{\star}:=l_{a}^{\star} \circ P_{\star}, P_{\star} \cdot a:=P_{\star} \circ l_{a}^{\star} .
$$

The $H^{\mathcal{F}}$-action is the $H^{\mathcal{F}}$-adjoint action given by, for all $\xi \in H^{\mathcal{F}}$ and $P_{\star} \in$ $\operatorname{End}_{\mathbb{K}}\left(V_{\star}\right)$,

$$
\xi \triangleright_{\mathcal{F}} P_{\star}:=\xi_{1_{\mathcal{F}}} \triangleright \circ P_{\star} \circ S^{\mathcal{F}}\left(\xi_{2_{\mathcal{F}}}\right) \triangleright .
$$

In quadruple notation, these ${ }^{H^{\mathcal{F}}} A_{\star} \mathscr{A}_{A_{\star}}$-algebras are denoted by $\left(\operatorname{End}_{\mathbb{K}}\left(V_{\star}\right), \circ\right.$, $\left.\cdot, \boldsymbol{F}_{\mathcal{F}}\right)$ and $\left(\operatorname{End}_{B_{\star}}\left(V_{\star}\right), \circ, \cdot, \boldsymbol{F}_{\mathcal{F}}\right)$.

Theorem 4.2. Let $H$ be a Hopf algebra with twist $\mathcal{F} \in H \otimes H$, and let $A, B$ be two ${ }^{H} \mathscr{A}$-algebras and $V$ be an ${ }^{H}{ }_{A} \mathscr{M}_{B}$-module. The map

$$
\begin{aligned}
D_{\mathcal{F}}: \operatorname{End}_{\mathbb{K}}(V)_{\star} & \longrightarrow \operatorname{End}_{\mathbb{K}}\left(V_{\star}\right), \\
P & \longmapsto D_{\mathcal{F}}(P):=\left(\overline{\mathrm{f}}^{\alpha} \triangleright P\right) \circ \overline{\mathrm{f}}_{\alpha} \triangleright
\end{aligned}
$$

is an ${ }^{H^{\mathcal{F}}}{ }_{A_{\star}} \mathscr{A}_{A_{\star}}$-algebra isomorphism between $\left(\operatorname{End}_{\mathbb{K}}(V), \circ_{\star}, \star, \triangleright\right)$ and

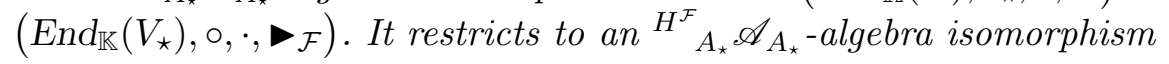

$$
D_{\mathcal{F}}: \operatorname{End}_{B}(V)_{\star} \longrightarrow \operatorname{End}_{B_{\star}}\left(V_{\star}\right)
$$

between $\left(\operatorname{End}_{B}(V), \circ_{\star}, \star, \triangleright\right)$ and $\left(\operatorname{End}_{B_{\star}}\left(V_{\star}\right), \circ, \cdot, \boldsymbol{\mathcal { F }}_{\mathcal{F}}\right)$.

Proof. The $H^{\mathcal{F}} \mathscr{A}$-algebra isomorphism (4.10) is given by the isomorphism $D_{\mathcal{F}}: \operatorname{End}_{\mathbb{K}}(V)_{\star} \rightarrow \operatorname{End}_{\mathbb{K}}(V)$ discussed in Example 3.15 .

The map $D_{\mathcal{F}}$ is an ${ }^{H^{\mathcal{F}}}{ }_{A_{\star}} \mathscr{A}_{A_{\star}}$-algebra isomorphism because (cf. Theorem 3.9, and recall the bimodule structures (4.7) and (4.8)) $D_{\mathcal{F}}(a \star P)=$ $D_{\mathcal{F}}\left(l_{a} \circ_{\star} P\right)=D_{\mathcal{F}}\left(l_{a}\right) \circ D_{\mathcal{F}}(P), D_{\mathcal{F}}(P \star a)=D_{\mathcal{F}}(P) \circ D_{\mathcal{F}}\left(l_{a}\right)$ and $D_{\mathcal{F}}\left(l_{a}\right)$ $=l_{a}^{\star}$. The last property follows from a short calculation

$$
D_{\mathcal{F}}\left(l_{a}\right)(v)=\left(\overline{\mathrm{f}}^{\alpha} \triangleright l_{a}\right)\left(\overline{\mathrm{f}}_{\alpha} \triangleright v\right)=l_{\overline{\mathrm{f}}^{\alpha} \triangleright a}\left(\overline{\mathrm{f}}_{\alpha} \triangleright v\right)=a \star v=l_{a}^{\star}(v) .
$$

In order to prove that $D_{\mathcal{F}}$ restricts to an isomorphism between the $H_{A_{\star}}^{\mathcal{F}} \mathscr{A}_{A_{\star}}$-subalgebras $\operatorname{End}_{B}(V)_{\star}$ and $\operatorname{End}_{B_{\star}}\left(V_{\star}\right)$ we show that for all $P \in$ $\operatorname{End}_{B}(V)$ we have $D_{\mathcal{F}}(P) \in \operatorname{End}_{B_{\star}}\left(V_{\star}\right)$, and that for all $P_{\star} \in \operatorname{End}_{B_{\star}}\left(V_{\star}\right)$ we have $D_{\mathcal{F}}^{-1}\left(P_{\star}\right) \in \operatorname{End}_{B}(V)$. Because of Remark 3.11 it is sufficient to prove the first statement, since the second follows from twisting back with $\mathcal{F}^{-1}$. 
The proof is short, for all $P \in \operatorname{End}_{B}(V), v \in V$ and $b \in B$,

$$
\begin{aligned}
D_{\mathcal{F}}(P)(v \star b) & =\left(\overline{\mathrm{f}}^{\alpha} \triangleright P\right)\left(\overline{\mathrm{f}}_{\alpha 1} \overline{\mathrm{f}}^{\beta} \triangleright v \cdot \overline{\mathrm{f}}_{\alpha_{2}} \overline{\mathrm{f}}_{\beta} \triangleright b\right) \\
& =\left(\overline{\mathrm{f}}_{1}^{\alpha} \overline{\mathrm{f}}^{\beta} \triangleright P\right)\left(\overline{\mathrm{f}}_{2}^{\alpha} \overline{\mathrm{f}}_{\beta} \triangleright v\right) \cdot\left(\overline{\mathrm{f}}_{\alpha} \triangleright b\right) \\
& =\overline{\mathrm{f}}^{\alpha} \triangleright\left(D_{\mathcal{F}}(P)(v)\right) \cdot\left(\overline{\mathrm{f}}_{\alpha} \triangleright b\right)=D_{\mathcal{F}}(P)(v) \star b
\end{aligned}
$$

where in the second line we used the twist cocycle property (3.4c) and the fact that $\xi \triangleright P \in \operatorname{End}_{B}(V)$, for all $\xi \in H$ and $P \in \operatorname{End}_{B}(V)$.

We call $D_{\mathcal{F}}(P): V_{\star} \rightarrow V_{\star}$ the deformation of the endomorphism $P: V \rightarrow V$ because $P$ is a map between undeformed modules and can be seen as an element of the undeformed ${ }^{H}{ }_{A} \mathscr{A}_{A}$-algebra $\operatorname{End}_{\mathbb{K}}(V)$. From this viewpoint $D_{\mathcal{F}}: \operatorname{End}_{\mathbb{K}}(V) \rightarrow \operatorname{End}_{\mathbb{K}}\left(V_{\star}\right)$ is a bijection from (the ${ }^{H}{ }_{A} \mathscr{A}_{A}$-algebra) $\operatorname{End}_{\mathbb{K}}(V)$ to (the ${ }^{H^{\mathcal{F}}} A_{\star} \mathscr{A}_{A_{\star}}$-algebra) $\operatorname{End}_{\mathbb{K}}\left(V_{\star}\right)$. Actually, since $\operatorname{End}_{\mathbb{K}}(V)$ and $\operatorname{End}_{\mathbb{K}}\left(V_{\star}\right)$ are $\mathbb{K}$-modules, it is an isomorphism of $\mathbb{K}$-modules.

Left $\boldsymbol{A}$-linear endomorphisms. We have so far studied the deformations of the algebra $\operatorname{End}_{B}(V)$ of right $B$-linear endomorphisms, but we could as well have studied the deformations of the algebra ${ }_{A} E n d(V)$ of left $A$-linear endomorphisms of the ${ }^{H}{ }_{A} \mathscr{M}_{B}$-module $V$. These deformations are obtained by a "mirror" construction. A key point is that there is an isomorphism ${ }_{A} \operatorname{End}(V) \simeq \operatorname{End}_{A^{o p}}\left(V^{o p}\right)$ between left $A$-linear endomorphisms of a module $V$ and right $A^{o p}$-linear endomorphisms of the opposite module $V^{o p}$, where, as we detail below, $A^{o p}$ has the opposite product of $A$, and $V^{o p}$ the opposite module structure of $V$. Then, twist deformation of left $A$-linear endomorphisms in ${ }_{A} \operatorname{End}(V)$ can be studied via twist deformation of right $A^{o p}$-linear endomorphisms in $\operatorname{End}_{A^{o p}}\left(V^{o p}\right)$.

We recall that given a Hopf algebra $H=(H, \mu, \Delta, S, \varepsilon)$ (where with slight abuse of notation we also denoted by $H$ the $\mathbb{K}$-module structure underlying the Hopf algebra $H$ ) we have the Hopf algebras $H^{c o p}$ and $H_{o p}$ if the antipode $S$ is invertible. Explicitly, $H^{c o p}=\left(H, \mu, \Delta^{c o p}, S^{-1}, \varepsilon\right)$ is the Hopf algebra with the co-opposite coproduct $\Delta^{c o p}$ defined by, for all $\xi \in H$, $\Delta^{c o p}(\xi):=\xi_{1^{\text {cop }}} \otimes \xi_{2^{\text {cop }}}:=\xi_{2} \otimes \xi_{1}$, where $\Delta(\xi)=\xi_{1} \otimes \xi_{2} . H_{o p}=\left(H, \mu^{o p}, \Delta\right.$, $\left.S^{-1}, \varepsilon\right)$ is the Hopf algebra with the opposite product $\mu^{o p}$ defined by, for all $\xi, \zeta \in H, \mu^{o p}(\xi \otimes \zeta)=\zeta \xi$. Even if the antipode $S$ is not invertible we have the Hopf algebra $H_{o p}^{c o p}=\left(H, \mu^{o p}, \Delta^{c o p}, S, \varepsilon\right)$. For a simpler mirror construction we are going to assume invertibility of the antipode in what follows. Notice that, in particular, quasitriangular Hopf algebras have an invertible antipode.

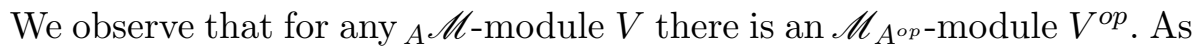
$\mathbb{K}$-modules $V=V^{o p}$, the algebra $A^{o p}$ is the algebra with opposite product 
and its right action on $V^{o p}$ is given by $v{ }^{o p} a:=a \cdot v$. Similarly, for any $\mathscr{M}_{A}$-module $V$ there is an $A^{o p} \mathscr{M}$-module $V^{o p}$, and $\left(V^{o p}\right)^{o p}=V$.

Moreover, if we have an ${ }^{H} \mathscr{A}$-algebra $A$ then the opposite algebra $A^{o p}$ is an $H^{\text {cop }} \mathscr{A}$-algebra, where the Hopf algebra action is unchanged, i.e. $A^{o p}$ is the $H^{\text {cop }} \mathscr{A}$-algebra $\left(A, \mu^{o p}, \triangleright\right)$. This easily leads to the following

Lemma 4.3. Let $A, B$ be two ${ }^{H} \mathscr{A}$-algebras and $V$ be an ${ }_{A}{ }_{A} \mathscr{M}_{B}$-module. Then $A^{o p}, B^{o p}$ are ${ }^{H^{\text {cop }}} \mathscr{A}$-algebras and $V^{o p}$ is an ${ }^{H^{\text {cop }}}{ }_{B^{o p}} \mathscr{M}_{A^{o p} \text {-module, where }}$ the $H^{\text {cop }}$-actions on $A^{o p}, B^{o p}, V^{o p}$ are the same actions as the $H$-actions on $A, B, V$. Similarly if $E$ is an ${ }_{A} \mathscr{A}_{B}$-algebra, then $E^{o p}$ is an ${ }^{H^{c o p}} B^{\text {op }} \mathscr{A}_{A^{o p}-}$ algebra.

Proof. We here show as an illustrative example that the algebra $A^{o p}$ is an $H^{\text {cop }} \mathscr{A}$-algebra. For all $\xi \in H$ and $a, \tilde{a} \in A$,

$$
\begin{aligned}
\xi \triangleright\left(\mu^{o p}(a \otimes \tilde{a})\right) & =\xi \triangleright(\tilde{a} a)=\left(\xi_{1} \triangleright \tilde{a}\right)\left(\xi_{2} \triangleright a\right)=\mu^{o p}\left(\left(\xi_{2} \triangleright a\right) \otimes\left(\xi_{1} \triangleright \tilde{a}\right)\right) \\
& =\mu^{o p}\left(\left(\xi_{1^{c o p}} \triangleright a\right) \otimes\left(\xi_{2^{c o p}} \triangleright \tilde{a}\right)\right) .
\end{aligned}
$$

The remaining statements are similarly proven.

We apply these observations to the algebra of endomorphisms of the module $V$ and obtain

Proposition 4.4. Let $A, B$ be two ${ }^{H} \mathscr{A}$-algebras and $V$ an ${ }^{H}{ }_{A} \mathscr{M}_{B}$-module. Then $\left({ }_{A} \operatorname{End}(V)\right)^{o p}$ is an ${ }_{B} \mathscr{A}_{B}$-algebra, i.e. more explicitly, $\left({ }_{A} \operatorname{End}(V), o^{o p}\right.$, $\left.{ }^{. o p},{ }^{c o p}\right)$ is an ${ }_{B} \mathscr{A}_{B}$-algebra, where the left $H$-action, called ${ }^{\text {cop }}$ adjoint action, is given by, for all $\xi \in H$ and $P \in{ }_{A} \operatorname{End}(V)$,

$$
\xi{ }^{c o p} P:=\xi_{2} \triangleright \circ P \circ S^{-1}\left(\xi_{1}\right) \triangleright .
$$

The B-bimodule structure is given by, for all $b \in B$ and $P \in{ }_{A} \operatorname{End}(V)$,

$$
b^{o p} P=P \circ r_{b}, P{ }^{o p} b=r_{b} \circ P,
$$

where for all $v \in V, r_{b}(v)=v \cdot b$.

Proof. The hypothesis implies that $A^{o p}$ and $B^{o p}$ are ${ }^{H^{\text {cop }}} \mathscr{A}$-algebras and

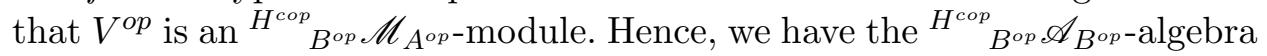
$\left(\operatorname{End}_{A^{o p}}\left(V^{o p}\right), \circ, \cdot, \triangleright^{c o p}\right)$, where the $H^{c o p}$-adjoint action is $\xi{ }^{c o p} P=\xi_{1^{c o p}} \triangleright$ 
$\circ P \circ S^{c o p}\left(\xi_{2^{\text {cop }}}\right) \triangleright$ and the $B^{o p}$-actions are

$$
P \cdot b=P \circ l_{b}^{B^{o p}}=P \circ r_{b}, b \cdot P=l_{b}^{B^{o p}} \circ P=r_{b} \circ P,
$$

where by definition $l^{B^{o p}}: B^{o p} \rightarrow \operatorname{End}_{A^{o p}}\left(V^{o p}\right)$, with $l_{b}^{B^{o p}}(v)=b \cdot{ }^{o p} v=v \cdot b$. Because of Lemma 4.3 we equivalently have the opposite ${ }_{B} \mathscr{A}_{B}$-algebra $\left(\operatorname{End}_{A^{o p}}\left(V^{o p}\right), \circ^{o p},{ }^{o p},{ }^{c o p}\right)$. The thesis holds because there is a canonical $\mathbb{K}$-module isomorphism

$$
\operatorname{End}_{A^{o p}}\left(V^{o p}\right) \simeq{ }_{A} \operatorname{End}(V)
$$

given by the identity map. Indeed, if $P \in \operatorname{End}_{A^{o p}}\left(V^{o p}\right)$ then, for all $a \in A$ and $v \in V$,

$$
P(a \cdot v)=P\left(v \cdot{ }^{o p} a\right)=P(v) \cdot{ }^{o p} a=a \cdot P(v)
$$

hence $P \in{ }_{A} \operatorname{End}(V)$, and vice versa. Thus, $\left({ }_{A} \operatorname{End}(V), \circ^{o p},{ }^{o p},{ }^{c o p}\right)$ is an ${ }_{B} \mathscr{A}_{B}$-algebra.

The appearance in Proposition 4.4 of the opposite composition product $P{ }^{\circ}$ op $Q=Q \circ P$ is naturally explained letting the left $A$-linear endomorphism $P$ act from the right to the left, $(v) \overleftarrow{P}:=P(v)$. Then we have $((v) \overleftarrow{P}) \overleftarrow{Q}=$ $Q(P(v))=(Q \circ P)(v)=(P \circ o p Q)(v)=(v) \overleftarrow{P \circ o p} Q$.

We now use the isomorphism ${ }_{A} \operatorname{End}(V) \simeq \operatorname{End}_{A^{o p}}\left(V^{o p}\right)$ between left $A$ linear and right $A^{o p}$-linear endomorphisms in order to construct a deformation map $P \mapsto D_{\mathcal{F}}^{c o p}(P)$ from left $A$-linear to left $A_{\star}$-linear endomorphisms. The map $D_{\mathcal{F}}^{c o p}$ is induced from a deformation map of right $A^{o p}$-linear endomorphism that is constructed following Theorem 4.2.

Let $H$ be a Hopf algebra with twist $\mathcal{F} \in H \otimes H$. Let also $A, B$ be two ${ }^{H} \mathscr{A}$-algebras and $V$ an ${ }^{H}{ }_{A} \mathscr{M}_{B}$-module, so that $\left({ }_{A} \operatorname{End}(V)\right)^{o p}$ is an ${ }_{B} \mathscr{A}_{B^{-}}$ algebra, it reads $\left({ }_{A} \operatorname{End}(V), \circ^{o p},{ }^{o p},{ }^{c o p}\right)$. Then the canonical constructions of Theorem 3.4 and of Theorem 3.5 lead to the deformed ${ }^{H^{\mathcal{F}}} \mathscr{A}$-algebras $A_{\star}, B_{\star}$, the deformed ${ }^{H^{\mathcal{F}}}{ }_{A_{\star}} \mathscr{M}_{B_{\star}}$-module $V_{\star}$ and the deformed ${ }^{H^{\mathcal{F}}}{ }_{B_{\star}} \mathscr{A}_{B_{\star}-}$ algebra $\left({ }_{A} \operatorname{End}(V)\right)_{\star}^{o p}$. This latter one explicitly reads $\left({ }_{A} \operatorname{End}(V),\left(\circ^{o p}\right)_{\star}\right.$, $\left.\left({ }^{o p}\right)_{\star},{ }^{c o p}\right)$, where $\left(\circ^{o p}\right)_{\star}$ and $(. o p)_{\star}$ are the $\star$-products constructed from ${ }^{o p}$ and the twist $\mathcal{F}$ (respectively ${ }^{o p}$ and the twist $\mathcal{F}$ ). For example, for $P, Q \in{ }_{A} \operatorname{End}(V), P\left(\circ^{o p}\right)_{\star} Q=\left(\overline{\mathrm{f}}^{\alpha}{ }^{c o p} P\right) \circ^{o p}\left(\overline{\mathrm{f}}_{\alpha}{ }^{c o p} Q\right)$.

Another deformation of $\left({ }_{A} \operatorname{End}(V)\right)^{o p}$ is achieved by applying the construction of Proposition 4.4 to the deformed $H^{\mathcal{F}} \mathscr{A}$-algebras $A_{\star}, B_{\star}$ and the deformed ${ }^{H^{\mathcal{F}}}{ }_{\star} \mathscr{M}_{B_{\star}}$-module $V_{\star}$. We thus obtain the ${ }_{B_{\star}}^{H^{\mathcal{F}}} \mathscr{A}_{B_{\star}}$-algebra 
$\left(A_{\star} \operatorname{End}\left(V_{\star}\right)\right)^{o p}$, or more explicitly $\left(A_{\star} \operatorname{End}\left(V_{\star}\right), \circ^{o p},{ }^{o p}, \mathcal{F}^{c o p}\right)$. Notice in particular that the $B_{\star}$-bimodule structure is given by, for all $b \in B_{\star}$ and $P_{\star} \in$ $A_{\star} \operatorname{End}\left(V_{\star}\right)$,

$$
b \cdot{ }^{o p} P_{\star}=P_{\star} \circ r_{b}^{\star}, P_{\star} \cdot{ }^{o p} b=r_{b}^{\star} \circ P_{\star},
$$

where, for all $v \in V_{\star}, r_{b}^{\star}(v)=v \star b$.

Theorem 4.5. Let $H$ be a Hopf algebra with twist $\mathcal{F} \in H \otimes H$, and let $A, B$ be two ${ }^{H} \mathscr{A}$-algebras and $V$ be an ${ }^{H}{ }_{A} \mathscr{M}_{B}$-module. The map

$$
\begin{aligned}
D_{\mathcal{F}}^{c o p}:\left({ }_{A} \operatorname{End}(V)\right)_{\star}^{o p} & \longrightarrow\left(A_{\star} \operatorname{End}\left(V_{\star}\right)\right)^{o p} \\
P & \longmapsto D_{\mathcal{F}}^{c o p}(P):=\left(\overline{\mathrm{f}}_{\alpha} \triangleright^{c o p} P\right) \circ \overline{\mathrm{f}}^{\alpha} \triangleright
\end{aligned}
$$

is an ${ }^{H^{\mathcal{F}}}{ }_{B_{\star}} \mathscr{A}_{B_{\star}}$-algebra isomorphism between the two deformations of left $A$ linear endomorphisms $\left({ }_{A} \operatorname{End}(V),\left({ }^{o p}\right)_{\star},\left(.{ }^{o p}\right)_{\star},{ }^{c o p}\right)$ and $\left(A_{\star} \operatorname{End}\left(V_{\star}\right), \circ^{o p}\right.$, $\left.{ }^{o p}, \boldsymbol{F}^{c o p}\right)$.

Proof. From Lemma 4.3 we have the ${ }^{H^{c o p}} \mathscr{A}$-algebras $A^{o p}, B^{o p}$ and the $H^{\text {cop }}{ }^{\text {op }} \mathscr{M}_{A^{o p}-\text { module }} V^{o p}$. Now notice that if $\mathcal{F}$ is a twist of $H$, then $\mathcal{F}^{\text {cop }}:=$ $\mathcal{F}_{21}$ is a twist of $H^{c o p}$. From Proposition 4.1 and the Theorems 3.4 and 3.5 we then have that

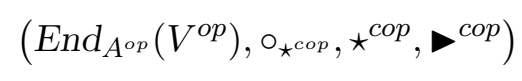

and

$$
\left(\operatorname{End}_{\left(A^{o p}\right)_{\star}{ }^{c o p}}\left(\left(V^{o p}\right)_{\star c o p}\right), \circ, \cdot,{ }^{c o p} \mathcal{F}^{c o p}\right)
$$

are ${ }^{\left(H^{c o p}\right)^{\mathcal{F}^{c o p}}\left(B^{o p}\right)_{\star}{ }_{\star c o p}} \mathscr{A}\left(B^{o p}\right)_{\star}{ }_{\star}$-ap -algebras. We denoted by $\star^{\text {cop }}$ the $\star$-product

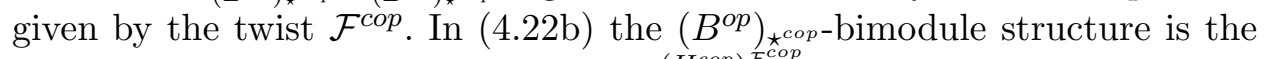
canonical one obtained from the $\left(H^{\text {cop }}\right)^{\mathcal{F}^{c o p}} \mathscr{A}$-algebra homomorphism $l^{\left(B^{o p}\right)_{\star c o p}}:\left(B^{o p}\right)_{\star_{c o p}} \rightarrow \operatorname{End}_{\left(A^{o p}\right)_{\star} c o p}\left(\left(V^{o p}\right)_{\star^{c o p}}\right)$. We use the short notation $\left(\operatorname{End}_{A^{o p}}\left(V^{o p}\right)\right)_{\star^{c o p}}$ for $(4.22 \mathrm{a})$ and $\operatorname{End}_{\left(A^{o p}\right)_{\star} c o p}\left(\left(V^{o p}\right)_{\star^{c o p}}\right)$ for $(4.22 \mathrm{~b})$. Now Theorem 4.2 implies the ${ }^{\left(H^{c o p}\right)^{\mathcal{F}^{c o p}}\left(B^{o p}\right)_{\star} \text { cop }} \mathscr{A}_{\left(B^{o p}\right)_{\star} \text { cop }}$-algebra isomorphism

$$
\begin{aligned}
& D_{\mathcal{F} c o p}:\left(\operatorname{End}_{A^{o p}}\left(V^{o p}\right)\right)_{\star^{c o p}} \longrightarrow \operatorname{End}_{\left(A^{o p}\right)_{\star} c o p}\left(\left(V^{o p}\right)_{\star_{\star}^{c o p}}\right) \text {, } \\
& P \longmapsto\left(\overline{\mathrm{f}}_{\alpha}{ }^{c o p} P\right) \circ \overline{\mathrm{f}}^{\alpha} \triangleright \text {. }
\end{aligned}
$$

Observe that

$$
\left(A^{o p}\right)_{\star c o p}=\left(A_{\star}\right)^{o p}, \quad\left(B^{o p}\right)_{\star c o p}=\left(B_{\star}\right)^{o p}, \quad\left(V^{o p}\right)_{\star} c o p=\left(V_{\star}\right)^{o p},
$$


as well as

$$
\left(H^{c o p}\right)^{\mathcal{F}^{c o p}}=\left(H^{\mathcal{F}}\right)^{c o p}, \quad \triangleright^{c o p}{ }_{\mathcal{F} c o p}=\triangleright_{\mathcal{F}}^{c o p}
$$

If we now consider an ${ }_{A}^{A} \mathscr{A}_{B}$-algebra $E$, so that $E^{o p}$ is an ${ }^{\text {cop }} B^{\text {op }} \mathscr{A}_{A^{\text {op }}}$ algebra, then the $\left(H^{c o p}\right)^{\mathcal{F}^{c o p}}\left(B^{o p}\right)_{\star}{ }_{\star}^{c o p} \mathscr{A}_{\left(A^{o p}\right)_{\star} \text { cop }}$-algebra $\left(E^{o p}\right)_{\star}$ cop is equal to

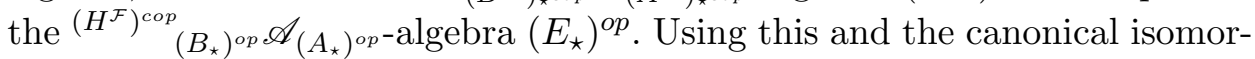
phism $\operatorname{End}_{A^{o p}}\left(V^{o p}\right) \simeq{ }_{A} \operatorname{End}(V)$ we find

$$
\left(\operatorname{End}_{A^{o p}}\left(V^{o p}\right)\right)_{\star^{c o p}} \simeq\left({ }_{A} \operatorname{End}(V)\right)_{\star^{c o p}}=\left(\left({ }_{A} \operatorname{End}(V)\right)^{o p}\right)_{\star}^{o p} .
$$

We also have that

$$
\operatorname{End}_{\left(A^{o p}\right)_{\star} c o p}\left(\left(V^{o p}\right)_{\star}^{c o p}\right)=\operatorname{End}_{\left(A_{\star}\right)^{o p}}\left(\left(V_{\star}\right)^{o p}\right) \simeq A_{\star} \operatorname{End}\left(V_{\star}\right)
$$

The bimodule structures in the first equality in $(4.25 \mathrm{~b})$ are identified via the

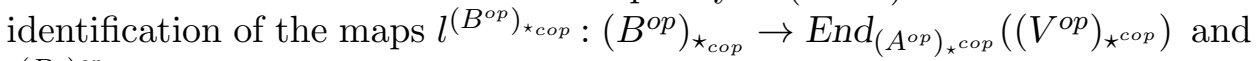

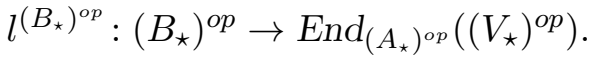

The proof of the theorem follows by noting that the isomorphism defined in (4.23) canonically induces on the opposites of the modules in (4.25) the ${ }_{B_{\star}}^{H_{\star}} \mathscr{A}_{B_{\star}}$-algebra isomorphism $D_{\mathcal{F}}^{c o p}$ defined in (4.21).

We call $D_{\mathcal{F}}^{c o p}(P): V_{\star} \rightarrow V_{\star}$ the left deformation of the endomorphism $P$ : $V \rightarrow V$.

\subsection{Deformation of homomorphisms}

Let $H$ be a Hopf algebra, $A, B$ be two ${ }^{H} \mathscr{A}$-algebras and $V$ be an ${ }^{H}{ }_{A} \mathscr{M}$ module. Then, as seen in the previous subsection, $\operatorname{End}_{\mathbb{K}}(V)$ is an ${ }^{H} \mathscr{A}$-algebra, where the $H$-action is given by the $H$-adjoint action $\downarrow$. The algebra structure

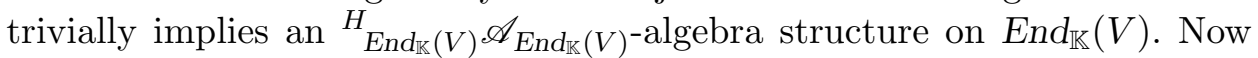
because of the ${ }^{H} \mathscr{A}$-algebra homomorphism $l: A \rightarrow \operatorname{End}_{\mathbb{K}}(V)$ (cf. proof of Proposition 4.1) we have that $\operatorname{End}_{\mathbb{K}}(V)$ is an ${ }^{H_{A}} \mathscr{A}_{A}$-algebra.

We here consider the $\mathbb{K}$-module $\operatorname{Hom}_{\mathbb{K}}(V, W)$ of $\mathbb{K}$-linear maps from the ${ }^{H}{ }_{A} \mathscr{M}$-module $V$ to the ${ }^{H}{ }_{A} \mathscr{M}$-module $W$. In this case we immediately

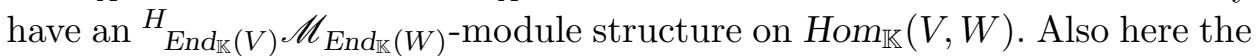
$H$-action is given by the $H$-adjoint action $\boldsymbol{~}$. Because of the ${ }^{H} \mathscr{A}$-algebra homomorphisms $l: A \rightarrow \operatorname{End}_{\mathbb{K}}(V)$ and $l: A \rightarrow \operatorname{End}_{\mathbb{K}}(W)$ we similarly obtain an ${ }^{H}{ }_{A} \mathscr{M}_{A}$-module structure on $\operatorname{Hom}_{\mathbb{K}}(V, W)$, explicitly $\left(\operatorname{Hom}_{\mathbb{K}}(V, W), \cdot, \boldsymbol{}\right)$. 
Furthermore, similarly to Proposition 4.1, we have that if $V, W$ are ${ }_{A}{ }_{A} \mathscr{M}_{B^{-}}$ modules, then the $\mathbb{K}$-submodule $\operatorname{Hom}_{B}(V, W)$ of right $B$-linear homomorphisms forms an ${ }_{A}{ }_{A} \mathscr{M}_{A}$-submodule of $\operatorname{Hom}_{\mathbb{K}}(V, W)$. The ${ }^{{ }_{A}} \mathscr{M}_{A}$-module structure of $\operatorname{Hom}_{B}(V, W)$ in the case $B=A$ will be a key ingredient in order to study tensor products over $A$ of homomorphisms, see Section 5.4.

Remark 4.6. We later also encounter the situation where $V$ is only an ${ }^{H} \mathscr{M}_{B}$-module while $W$ is an ${ }^{H}{ }_{A} \mathscr{M}_{B}$-module. In this case we similarly have

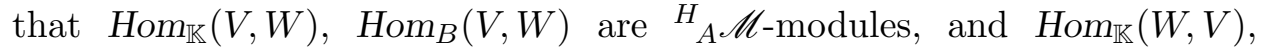
$\operatorname{Hom}_{B}(W, V)$ are ${ }^{H} \mathscr{M}_{A}$-modules.

The results of the previous subsection concerning the deformation of endomorphisms generalize to the case of homomorphisms. We present only the main theorems and omit the proofs that are easily obtained following the corresponding ones for endomorphisms.

Theorem 4.7. Let $H$ be a Hopf algebra with twist $\mathcal{F} \in H \otimes H$, and let $A, B$ be two ${ }^{H} \mathscr{A}$-algebras and $V, W$ be two ${ }_{A}{ }_{A} \mathscr{M}_{B}$-modules. The map

$$
\begin{aligned}
D_{\mathcal{F}}: \operatorname{Hom}_{\mathbb{K}}(V, W)_{\star} & \longrightarrow \operatorname{Hom}_{\mathbb{K}}\left(V_{\star}, W_{\star}\right), \\
P & \longmapsto D_{\mathcal{F}}(P):=\left(\overline{\mathrm{f}}^{\alpha} \triangleright P\right) \circ \overline{\mathrm{f}}_{\alpha} \triangleright
\end{aligned}
$$

is an ${ }^{H^{\mathcal{F}}}{ }_{A_{\star}} \mathscr{M}_{A_{\star}}$-module isomorphism between $\left(\operatorname{Hom}_{\mathbb{K}}(V, W), \star, \triangleright\right)$ and

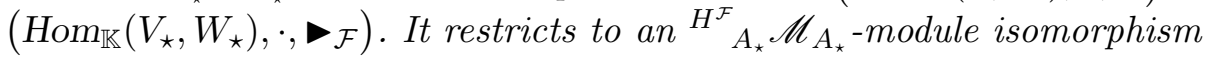

$$
D_{\mathcal{F}}: \operatorname{Hom}_{B}(V, W)_{\star} \longrightarrow \operatorname{Hom}_{B_{\star}}\left(V_{\star}, W_{\star}\right)
$$

between $\left(\operatorname{Hom}_{B}(V, W), \star, \triangleright\right)$ and $\left(\operatorname{Hom}_{B_{\star}}\left(V_{\star}, W_{\star}\right), \cdot, \boldsymbol{F}_{\mathcal{F}}\right)$.

We call $D_{\mathcal{F}}(P): V_{\star} \rightarrow W_{\star}$ the deformation of the homomorphism $P$ : $V \rightarrow W$.

Left $\boldsymbol{A}$-linear homomorphisms. The set $\left({ }_{A} \operatorname{Hom}(V, W)\right)^{o p}$ of left $A$ linear homomorphisms, between the ${ }^{H}{ }_{A} \mathscr{M}_{B}$-modules $V$ and $W$, carries an ${ }_{B} \mathscr{M}_{B}$-module structure, explicitly $\left({ }_{A} \operatorname{Hom}(V, W),{ }^{o p},{ }^{c o p}\right)$, where, for all $b \in B, \xi \in H$ and $P \in{ }_{A} \operatorname{Hom}(V, W), \xi{ }^{c o p} P:=\xi_{2} \triangleright \circ P \circ S^{-1}\left(\xi_{1}\right) \triangleright, b . o p$ $P=P \circ r_{b}$ and $P .{ }^{\circ o p} b=r_{b} \circ P$.

Up to isomorphism there is just one deformed module of left $A$-linear homomorphisms. 
Theorem 4.8. Let $H$ be a Hopf algebra with twist $\mathcal{F} \in H \otimes H$, and let $A, B$ be two ${ }^{H} \mathscr{A}$-algebras and $V, W$ be two ${ }^{H}{ }_{A} \mathscr{M}_{B}$-modules. The map

$$
\begin{aligned}
D_{\mathcal{F}}^{c o p}:\left({ }_{A} \operatorname{Hom}(V, W)\right)^{o p} & \longrightarrow\left(A_{\star} \operatorname{Hom}\left(V_{\star}, W_{\star}\right)\right)^{o p} \\
P & \longmapsto D_{\mathcal{F}}^{c o p}(P):=\left(\overline{\mathrm{f}}_{\alpha} \triangleright^{c o p} P\right) \circ \overline{\mathrm{f}}^{\alpha} \triangleright
\end{aligned}
$$

is an ${ }^{H^{\mathcal{F}}}{ }_{B_{\star}} \mathscr{M}_{B_{\star}}$-module isomorphism between the two deformations of left $A$ linear homomorphisms $\left({ }_{A} \operatorname{Hom}(V, W),\left({ }^{o p}\right)_{\star},{ }^{c o p}\right)$ and $\left(A_{\star} \operatorname{Hom}\left(V_{\star}, W_{\star}\right),{ }^{o p}\right.$, $\left.\boldsymbol{\mathcal { F }}^{\mathrm{cop}}\right)$.

We call $D_{\mathcal{F}}^{c o p}(P): V_{\star} \rightarrow W_{\star}$ the left deformation of the homomorphism $P: V \rightarrow W$.

Example 4.9. Let $A$ be an ${ }^{H} \mathscr{A}$-algebra and $V$ be an ${ }^{H}{ }_{A} \mathscr{M}_{A}$-module. The dual module of $V$ is defined by $V^{\prime}:=\operatorname{Hom}_{A}(V, A)$. Since $A$ can be regarded as an ${ }^{H}{ }_{A} \mathscr{M}_{A}$-module we have that $V^{\prime}$ is also an ${ }^{H} \mathscr{M}_{A}$-module. Let $\mathcal{F} \in$ $H \otimes H$ be a twist of $H$ and consider the deformed $H^{\mathcal{F}} \mathscr{A}$-algebra $A_{\star}$ and the deformed ${ }^{H^{\mathcal{F}}}{ }_{A_{\star}} \mathscr{M}_{A_{\star}}$-module $V_{\star}$. We have two possible deformations of the dual module: $V^{\prime}{ }_{\star}=\operatorname{Hom}_{A}(V, A)_{\star}$ and $V_{\star}{ }^{\prime}=\operatorname{Hom}_{A_{\star}}\left(V_{\star}, A_{\star}\right)$. They both are ${ }^{H^{\mathcal{F}}}{ }_{A_{\star}} \mathscr{M}_{A_{\star}}$-modules. Due to Theorem 4.7 there is an ${ }^{H^{\mathcal{F}}}{ }_{A_{\star}} \mathscr{M}_{A_{\star}}$-module isomorphism $V^{\prime}{ }_{\star} \simeq V_{\star}{ }^{\prime}$. In words, dualizing the deformed module is (up to the canonical isomorphism $D_{\mathcal{F}}$ ) equivalent to deforming the dual one. Similar statements hold true for the left $A$-linear dual ${ }^{\prime} V:=\left({ }_{A} \operatorname{Hom}(V, A)\right)^{o p}$ and its deformations according to Theorem 4.8.

\subsection{Categorical formulation}

Developing the results of Theorem 3.16 we provide a categorical formulation of the investigations of the present section. We first define the category $\left({ }_{A}{ }_{A} \mathscr{M}_{B}, H_{0} m_{B}, \circ\right)$. An object in $\left({ }_{A} \mathscr{M}_{B}, H o m_{B}, \circ\right)$ is an ${ }_{A} \mathscr{M}_{B}$-module $V$ and a morphism between two objects $V, W$ in $\left({ }_{A} \mathscr{M}_{B}, H_{B} m_{B}, \circ\right)$ is a right $B$-linear map $P: V \rightarrow W$, i.e. $P \in \operatorname{Hom}_{B}(V, W)$. The composition of morphisms is the usual composition $\circ$. Given a twist $\mathcal{F} \in H \otimes H$ of the Hopf algebra $H$ we then define two other categories, $\left({ }_{A}^{H} \mathscr{M}_{B}, H_{0} m_{B}, \circ_{\star}\right)$

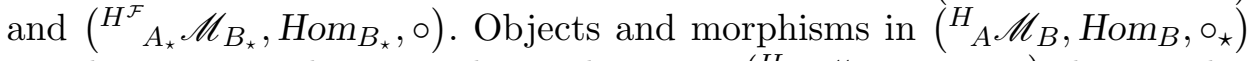
are the same as objects and morphisms in $\left({ }_{A} \mathscr{M}_{B}, H_{0} m_{B}, \circ\right)$, but in this category the composition of morphisms is given by the $\star$-composition $\circ_{\star}$. An

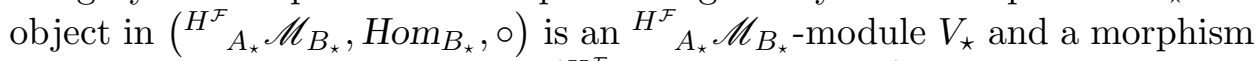
between two objects $V_{\star}, W_{\star}$ in $\left({ }^{H^{\mathcal{F}}}{ }_{A_{\star}} \mathscr{M}_{B_{\star}}, H_{0^{*}} m_{B_{\star}}, \circ\right)$ is a right $B_{\star}$-linear 
$\operatorname{map} P_{\star}: V_{\star} \rightarrow W_{\star}$, i.e. $P_{\star} \in H_{o m} B_{\star}\left(V_{\star}, W_{\star}\right)$. The composition of morphisms is the usual composition $\circ$.

Theorem 4.10. Let $H$ be a Hopf algebra with twist $\mathcal{F} \in H \otimes H$. Then there is a functor from $\left({ }^{H} \mathscr{M}_{B}, H_{o m}, \circ_{\star}\right)$ to $\left({ }_{A_{\star}}^{H^{\mathcal{F}}} \mathscr{M}_{B_{\star}}, H_{0 m} B_{\star}, \circ\right)$. It maps any ${ }^{H}{ }_{A} \mathscr{M}_{B}$-module $V$ to the twist deformed ${ }^{H^{\mathcal{F}}}{ }_{A_{\star}} \mathscr{M}_{B_{\star}}$-module $V_{\star}$ (cf. Theorem 3.5), and any morphism $P \in \operatorname{Hom}_{B}(V, W)$ to the morphism $D_{\mathcal{F}}(P) \in$ Hom $_{B_{\star}}\left(V_{\star}, W_{\star}\right)$ (cf. Theorem 4.7).

Furthermore, the categories $\left({ }^{H}{ }_{A} \mathscr{M}_{B}, H_{o m}, \circ_{\star}\right)$ and $\left({ }^{H^{\mathcal{F}}}{ }_{A_{\star}} \mathscr{M}_{B_{\star}}, H_{o m}{ }_{B_{\star}}\right.$, ○) are equivalent.

Proof. The proof of this theorem follows similar steps as that of Theorem 3.16 .

Left $\boldsymbol{A}$-linear morphisms. For completeness we state without proof the corresponding theorem for left $A$-linear maps.

We first define the category $\left({ }^{H}{ }_{A} \mathscr{M}_{B},{ }_{A} H o m, o^{o p}\right)$. An object in $\left({ }^{H} \mathscr{M}_{B}\right.$, ${ }_{A} H o m, \circ^{o p}$ ) is an ${ }_{A} \mathscr{M}_{B}$-module $V$ and a morphism between two objects $V, W$ in $\left({ }_{A} \mathscr{M}_{B},{ }_{A} H o m,{ }^{o p}\right)$ is a left $A$-linear map $P: V \rightarrow W$, i.e. $P \in$ ${ }_{A} \operatorname{Hom}(V, W)$. The composition of morphisms $\circ^{o p}$ is described after Proposition 4.4 .

Given a twist $\mathcal{F} \in H \otimes H$ of the Hopf algebra $H$ we then define two other categories, $\left({ }_{A} \mathscr{M}_{B},{ }_{A} H o m,\left(\circ^{o p}\right)_{\star}\right)$ and $\left({ }^{H^{\mathcal{F}}}{ }_{A_{\star}} \mathscr{M}_{B_{\star}}, A_{\star} H o m, o^{o p}\right)$. The objects and morphisms in $\left({ }^{H} \mathscr{M}_{B},{ }_{A} H o m,\left({ }^{o p}\right)_{\star}\right)$ are the same as objects and morphisms in $\left({ }_{A} \mathscr{M}_{B},{ }_{A} H o m, o^{o p}\right)$, but in this category the composition of morphisms is given by $\left(\circ^{o p}\right)_{\star}$, i.e. for two composable morphisms $P\left(\circ^{o p}\right)_{\star} Q=\left(\overline{\mathrm{f}}^{\alpha}{ }^{c o p} P\right) \circ^{o p}\left(\overline{\mathrm{f}}_{\alpha}{ }^{c o p} Q\right)$. An object in $\left({ }^{H^{\mathcal{F}}} A_{\star} \mathscr{M}_{B_{\star}}, A_{\star} H o m\right.$, $\left.\circ^{o p}\right)$ is an ${ }_{A_{\star}}^{H^{\mathcal{F}}} \mathscr{M}_{B_{\star}}$-module $V_{\star}$ and a morphism between two objects $V_{\star}, W_{\star}$ in $\left({ }^{H^{\mathcal{F}}} A_{\star} \mathscr{M}_{B_{\star}, A_{\star}}\right.$ Hom, $\left.\circ^{o p}\right)$ is a left $A_{\star}$-linear map $P_{\star}: V_{\star} \rightarrow W_{\star}$, i.e. $P_{\star} \in$ $A_{\star} \operatorname{Hom}\left(V_{\star}, W_{\star}\right)$. The composition of morphisms is $\circ^{o p}$.

Theorem 4.11. Let $H$ be a Hopf algebra with twist $\mathcal{F} \in H \otimes H$. Then there is functor from $\left({ }^{H} \mathscr{M}_{B},{ }_{A} H o m,\left(\circ^{o p}\right)_{\star}\right)$ to $\left({ }^{H^{\mathcal{F}}}{ }_{A_{\star}} \mathscr{M}_{B_{\star}}, A_{\star} H o m, o^{o p}\right)$. It maps any ${ }^{{ }_{A}} \mathscr{M}_{B}$-module $V$ to the twist deformed ${ }^{{ }^{H^{\mathcal{F}}}}{ }_{A_{\star}} \mathscr{M}_{B_{\star}}$-module $V_{\star}$ (cf. Theorem 3.5) and any morphism $P \in{ }_{A} \operatorname{Hom}(V, W)$ to the morphism $D_{\mathcal{F}}^{c o p}(P) \in A_{\star} \operatorname{Hom}\left(V_{\star}, W_{\star}\right)$ (cf. Theorem 4.8).

Furthermore, the categories $\left({ }^{H} \mathscr{M}_{B},{ }_{A} \mathrm{Hom},\left(\mathrm{O}^{o p}\right)_{\star}\right)$ and $\left({ }^{H^{\mathcal{F}}}{ }_{A_{\star}} \mathscr{M}_{B_{\star}}\right.$, $A_{\star}$ Hom, $\circ^{o p}$ ) are equivalent.

Remark 4.12. This is a generalization of the equivalence of categories obtained by Giaquinto and Zhang [19]. We can recover their equivalence 
by choosing the algebra $B=\mathbb{K}$ to be trivial and restricting the class of morphisms to $H$-equivariant (respectively $H^{\mathcal{F}}$-equivariant) and left $A$-linear (respectively left $A_{\star}$-linear) maps. Then the $\left(\circ^{o p}\right)_{\star}$ composition equals the ${ }^{o p}$ composition and the equivalence is between the categories $\left({ }^{H}{ }_{A} \mathscr{M},{ }_{A} H o m\right.$, $\left.\circ^{o p}\right)$ and $\left({ }^{H^{\mathcal{F}}}{ }_{\star} \mathscr{M}, A_{\star} H o m, \circ^{o p}\right)$. Notice also that in this case the functor acts trivially on morphisms. We remark that the more general equivalence of Theorem 4.11 is not between the original category $\left({ }^{H} \mathscr{M}_{B},{ }_{A} H o m, \circ^{o p}\right)$ and $\left({ }^{H^{\mathcal{F}}}{ }_{A_{\star}} \mathscr{M}_{B_{\star}}, A_{\star} H o m, \circ^{o p}\right)$, but between the original category with deformed composition law and the latter one.

\section{Tensor product structure and its deformation}

Let $H$ be a Hopf algebra. The tensor product $V \otimes W$ of two ${ }^{H} \mathscr{M}$-modules $V, W$ is again an ${ }^{H} \mathscr{M}$-module. The left $H$-action is defined using the coproduct, for all $\xi \in H, v \in V$ and $w \in W$,

$$
\xi \triangleright(v \otimes w):=\left(\xi_{1} \triangleright v\right) \otimes\left(\xi_{2} \triangleright w\right)
$$

and extended by $\mathbb{K}$-linearity to all $V \otimes W$.

We begin this section studying the lift of two $\mathbb{K}$-linear maps $P: V \rightarrow \widetilde{V}$, $Q: W \rightarrow \widetilde{W}$ to a tensor product map $V \otimes W \rightarrow \widetilde{V} \otimes \widetilde{W}$. The issue is that the construction has to be compatible with the $H$-action even if the maps we consider are in general only $H$-covariant (not $H$-equivariant). This requires extra structure on the Hopf algebra $H$. Indeed, in order to introduce a tensor product of $\mathbb{K}$-linear maps between ${ }^{H} \mathscr{M}$-modules that is compatible with the Hopf algebra action we require a braiding isomorphism on tensor products of ${ }^{H} \mathscr{M}$-modules. We therefore consider quasitriangular Hopf algebras. Next, we study the deformation of the tensor product of $\mathbb{K}$-linear maps and show that the deformation procedure is canonical.

In a later subsection, we shall focus on the restricted class of quasicommutative ${ }^{H} \mathscr{A}$-algebras and ${ }^{H} \mathscr{M}_{A}$-modules. The tensor product structure previously studied induces a tensor product structure over $A$. In particular the tensor product of right $A$-linear maps between quasi-commutative $H_{A} \mathscr{M}_{A}$-modules is compatible with the Hopf algebra action and is again a right $A$-linear map. Also the deformation of this tensor product is studied and shown to be canonical.

Finally, we consider the deformation map $D_{\mathcal{R}}$, that corresponds to the twist $\mathcal{F}=\mathcal{R}$, where $\mathcal{R}$ is the universal $\mathcal{R}$-matrix of the quasitriangular Hopf algebra $H$. The map $D_{\mathcal{R}}$ provides an isomorphism between right and left $A$-linear maps on strong quasi-commutative ${ }^{H}{ }_{A} \mathscr{M}_{A}$-modules. 


\subsection{Triangular and quasitriangular Hopf algebras}

A cocommutative Hopf algebra is a Hopf algebra where the coproduct is cocommutative, i.e., for all $\xi \in H, \Delta^{c o p}(\xi)=\Delta(\xi)$ or, using Sweedler's notation, $\xi_{2} \otimes \xi_{1}=\xi_{1} \otimes \xi_{2}$.

Definition 5.1. A quasi-cocommutative Hopf algebra $(H, \mathcal{R})$ is a Hopf algebra $H$ and an invertible element $\mathcal{R} \in H \otimes H$ (called universal $\mathcal{R}$-matrix) such that, for all $\xi \in H$,

$$
\Delta^{c o p}(\xi)=\mathcal{R} \Delta(\xi) \mathcal{R}^{-1} .
$$

The Hopf algebra is quasitriangular if moreover

$$
(\Delta \otimes \mathrm{id}) \mathcal{R}=\mathcal{R}_{13} \mathcal{R}_{23}, \quad(\mathrm{id} \otimes \Delta) \mathcal{R}=\mathcal{R}_{13} \mathcal{R}_{12} .
$$

The quasitriangular Hopf algebra $(H, \mathcal{R})$ is triangular if

$$
\mathcal{R}_{21}=\mathcal{R}^{-1},
$$

where $\mathcal{R}_{21}=\sigma(\mathcal{R}) \in H \otimes H$, with $\sigma$ the transposition map $\sigma(\xi \otimes \zeta)=\zeta \otimes \xi$, for all $\xi, \zeta \in H$.

For later use we write the property (5.2) and the inverse of properties (5.3) using Sweedler's notation and the notations $\mathcal{R}=R^{\alpha} \otimes R_{\alpha}, \mathcal{R}^{-1}=$ $\bar{R}^{\alpha} \otimes \bar{R}_{\alpha}$ (sum over $\alpha$ understood). For all $\xi \in H$,

$$
\begin{aligned}
\xi_{2} \otimes \xi_{1} & =R^{\alpha} \xi_{1} \bar{R}^{\beta} \otimes R_{\alpha} \xi_{2} \bar{R}_{\beta}, \\
\bar{R}_{1}^{\alpha} \otimes \bar{R}_{2}^{\alpha} \otimes \bar{R}_{\alpha} & =\bar{R}^{\alpha} \otimes \bar{R}^{\beta} \otimes \bar{R}_{\beta} \bar{R}_{\alpha}, \\
\bar{R}^{\alpha} \otimes \bar{R}_{\alpha_{1}} \otimes \bar{R}_{\alpha_{2}} & =\bar{R}^{\alpha} \bar{R}^{\beta} \otimes \bar{R}_{\alpha} \otimes \bar{R}_{\beta} .
\end{aligned}
$$

The triangular property reads $R_{\alpha} \otimes R^{\alpha}=\bar{R}^{\alpha} \otimes \bar{R}_{\alpha}$.

From (5.2) (with $\xi=R^{\alpha}$ ) and (5.3) it follows that quasitriangular $\mathcal{R}$ matrices satisfy the Yang-Baxter equation

$$
\mathcal{R}_{12} \mathcal{R}_{13} \mathcal{R}_{23}=\mathcal{R}_{23} \mathcal{R}_{13} \mathcal{R}_{12} .
$$

Further standard properties of quasitriangular $\mathcal{R}$-matrices are (see e.g. [24], Lemma 2.1.2)

$$
\begin{array}{cl}
(\varepsilon \otimes \mathrm{id}) \mathcal{R}=1, & (\text { id } \otimes \varepsilon) \mathcal{R}=1, \\
(S \otimes \mathrm{id}) \mathcal{R}=\mathcal{R}^{-1}, & (\text { id } \otimes S) \mathcal{R}^{-1}=\mathcal{R} .
\end{array}
$$


Notice that the properties (5.3), (5.6) and (5.7a) imply that $\mathcal{R}$ is a twist element of the Hopf algebra $H$. From property (5.2) it then follows that $H^{\mathcal{R}}=H^{c o p}$. The Hopf algebra $H^{\text {cop }}$ is quasitriangular with $\mathcal{R}$-matrix $\mathcal{R}^{\text {cop }}$ $=\mathcal{R}_{21}$.

Given two ${ }^{H} \mathscr{M}$-modules $V, W$ we have the tensor product ${ }^{H} \mathscr{M}$-modules $V \otimes W$ and $W \otimes V$ (see (5.1)). There is a natural isomorphism, called braiding, between these two tensor products; it is defined by

$$
\begin{aligned}
\tau_{\mathcal{R} W, V}: W \otimes V & \rightarrow V \otimes W, \\
w \otimes v & \mapsto \tau_{\mathcal{R} W, V}(w \otimes v)=\left(\bar{R}^{\alpha} \triangleright v\right) \otimes\left(\bar{R}_{\alpha} \triangleright w\right),
\end{aligned}
$$

$$
\begin{aligned}
\tau_{\mathcal{R} W, V}^{-1}: V \otimes W & \rightarrow W \otimes V, \\
v \otimes w & \mapsto \tau_{\mathcal{R} W, V}^{-1}(v \otimes w)=\left(R_{\alpha} \triangleright w\right) \otimes\left(R^{\alpha} \triangleright w\right) .
\end{aligned}
$$

and extended by $\mathbb{K}$-linearity to all $W \otimes V$ (and respectively $V \otimes W$ ). Quasicocommutativity of the coproduct (cf. (5.2)) implies that $\tau_{\mathcal{R}}$ and its inverse $\tau_{\mathcal{R}}^{-1}$ (for ease of notation we frequently omit the module indices) are ${ }^{H} \mathscr{M}$ module isomorphisms, i.e., for all $\xi \in H, v \in V, w \in W, \xi \triangleright\left(\tau_{\mathcal{R}}(w \otimes v)\right)=$ $\tau_{\mathcal{R}}(\xi \triangleright(w \otimes v)), \xi \triangleright\left(\tau_{\mathcal{R}}^{-1}(v \otimes w)\right)=\tau_{\mathcal{R}}^{-1}(\xi \triangleright(v \otimes w))$, or equivalently

$$
\xi \triangleright \tau_{\mathcal{R}}=\varepsilon(\xi) \tau_{\mathcal{R}}, \quad \xi \triangleright \tau_{\mathcal{R}}^{-1}=\varepsilon(\xi) \tau_{\mathcal{R}}^{-1} .
$$

From (5.3) it follows that on the triple tensor product $V \otimes W \otimes Z$ of ${ }^{H} \mathscr{M}$ modules $V, W, Z$ we have the braid relations

$$
\begin{aligned}
& \tau_{\mathcal{R} V \otimes W, Z}=\left(\tau_{\mathcal{R} V, Z} \otimes \operatorname{id}_{W}\right) \circ\left(\operatorname{id}_{V} \otimes \tau_{\mathcal{R} W, Z}\right) \\
& \tau_{\mathcal{R} V, W \otimes Z}=\left(\operatorname{id}_{W} \otimes \tau_{\mathcal{R} V, Z}\right) \circ\left(\tau_{\mathcal{R} V, W} \otimes \operatorname{id}_{Z}\right) .
\end{aligned}
$$

The first one, for example, states that flipping an element $z$ to the left of the element $v \otimes w$ is the same as first flipping $z$ to the left of $w$ and then the result to the left of $v$.

Example 5.2. The universal enveloping algebra $U \mathfrak{g}$ of a Lie algebra $\mathfrak{g}$ is a cocommutative Hopf algebra. Every cocommutative Hopf algebra $H$ has a triangular structure given by the $\mathcal{R}$-matrix $\mathcal{R}=1 \otimes 1$. Let $\mathcal{F}$ be a twist of this cocommutative Hopf algebra $H$, then the Hopf algebra $H^{\mathcal{F}}$ is triangular with $\mathcal{R}$-matrix $\mathcal{R}^{\mathcal{F}}=\mathcal{F}_{21} \mathcal{F}^{-1}$.

More in general, if $(H, \mathcal{R})$ is a quasitriangular (triangular) Hopf algebra, then $\left(H^{\mathcal{F}}, \mathcal{R}^{\mathcal{F}}:=\mathcal{F}_{21} \mathcal{R} \mathcal{F}^{-1}\right)$ is a quasitriangular (triangular) Hopf algebra. 


\subsection{Tensor product of $\mathbb{K}$-linear maps}

Given two $\mathbb{K}$-linear maps $P: V \rightarrow \widetilde{V}$ and $Q: W \rightarrow \widetilde{W}$ between $\mathbb{K}$-modules, the tensor product map $P \otimes Q: V \otimes W \rightarrow \widetilde{V} \otimes \widetilde{W}$ is the $\mathbb{K}$-linear map defined by, for all $v \in V$ and $w \in W$,

$$
(P \otimes Q)(v \otimes w):=P(v) \otimes Q(w),
$$

and extended to all $V \otimes W$ by $\mathbb{K}$-linearity. If $\widetilde{P}: \widetilde{V} \rightarrow \widehat{V}$ and $\widetilde{Q}: \widetilde{W} \rightarrow \widehat{W}$ are two further $\mathbb{K}$-linear maps, then we have the composition property

$$
(\widetilde{P} \otimes \widetilde{Q}) \circ(P \otimes Q)=(\widetilde{P} \circ P) \otimes(\widetilde{Q} \circ Q) .
$$

Let now $H$ be a Hopf algebra. We consider ${ }^{H} \mathscr{M}$-modules $V, W, \widetilde{V}, \widetilde{W}$ and the associated tensor product ${ }^{H} \mathscr{M}$-modules $V \otimes W$ and $\widetilde{V} \otimes \widetilde{W}$. The $\mathbb{K}$-modules of $\mathbb{K}$-linear maps $\operatorname{Hom}_{\mathbb{K}}(V, \widetilde{V}), \operatorname{Hom}_{\mathbb{K}}(W, \widetilde{W})$ and $\operatorname{Hom}_{\mathbb{K}}(V \otimes$ $W, \widetilde{V} \otimes \widetilde{W})$ are ${ }^{H} \mathscr{M}$-modules with the $H$-adjoint action. We study the action of $\xi \in H$ on the tensor product map (5.11). Using (5.1) and (5.12) we obtain

$$
\begin{aligned}
\xi \triangleright(P \otimes Q) & =\left(\xi_{1} \triangleright \otimes \xi_{2} \triangleright\right) \circ(P \otimes Q) \circ\left(S\left(\xi_{4}\right) \triangleright \otimes S\left(\xi_{3}\right) \triangleright\right) \\
& =\left(\xi_{1} \triangleright \circ P \circ S\left(\xi_{4}\right) \triangleright\right) \otimes\left(\xi_{2} \triangleright \circ Q \circ S\left(\xi_{3}\right) \triangleright\right) \\
& =\left(\xi_{1} \triangleright \circ P \circ S\left(\xi_{3}\right) \triangleright\right) \otimes\left(\xi_{2} \triangleright Q\right) .
\end{aligned}
$$

For a non-cocommutative Hopf algebra and $\mathbb{K}$-linear maps $Q$ that are not $H$ equivariant (i.e., $\xi \triangleright Q \neq \varepsilon(\xi) Q)$ this expression in general differs from $\left(\xi_{1}\right.$ $P) \otimes\left(\xi_{2} \triangleright Q\right)$. This shows that the tensor product of $\mathbb{K}$-linear maps (5.11) is in general incompatible with the ${ }^{H} \mathscr{M}$-module structure. This incompatibility can be understood as follows: Considering $\mathbb{K}$-linear maps as acting from left to right, the ordering on the left hand side of (5.11) is $P, Q, v, w$, while the ordering on the right hand side is $P, v, Q, w$, i.e. $v$ and $Q$ do not appear properly ordered in the definition (5.11). For a quasitriangular Hopf algebra $(H, \mathcal{R})$ this problem can be solved by defining a new tensor product of $\mathbb{K}$ linear maps.

Definition 5.3. Let $(H, \mathcal{R})$ be a quasitriangular Hopf algebra and $V, W, \widetilde{V}$, $\widetilde{W}$ be ${ }^{H} \mathscr{M}$-modules. The $\mathcal{R}$-tensor product of $\mathbb{K}$-linear maps is defined by, for all $P \in \operatorname{Hom}_{\mathbb{K}}(V, \widetilde{V})$ and $Q \in \operatorname{Hom}_{\mathbb{K}}(W, \widetilde{W})$,

$$
P \otimes_{\mathcal{R}} Q:=\left(P \circ \bar{R}^{\alpha} \triangleright\right) \otimes\left(\bar{R}_{\alpha} \triangleright Q\right) \in \operatorname{Hom}_{\mathbb{K}}(V \otimes W, \widetilde{V} \otimes \widetilde{W}),
$$

where $\otimes$ is defined in (5.11). 
This definition is related to the $H$-equivariant maps that appeared in [24], Corollary 9.3.16, and in [18], eq. (38). The tensor product perspective we consider in this paper leads to further investigations (starting from Theorem 5.4).

From Definition 5.3 it immediately follows that

$$
P \otimes_{\mathcal{R}} Q=(P \otimes \mathrm{id}) \circ\left(\bar{R}^{\alpha} \triangleright \otimes \bar{R}_{\alpha} \triangleright Q\right)=\left(P \otimes_{\mathcal{R}} \mathrm{id}\right) \circ\left(\mathrm{id} \otimes_{\mathcal{R}} Q\right)
$$

We see that the lift of $P \in \operatorname{Hom}_{\mathbb{K}}(V, \widetilde{V})$ to $\operatorname{Hom}_{\mathbb{K}}(V \otimes \widetilde{W}, \widetilde{V} \otimes \widetilde{W})$ is simply $P \otimes_{\mathcal{R}}$ id $=P \otimes \mathrm{id}$, while the lift of $Q$ is

$$
\operatorname{id} \otimes_{\mathcal{R}} Q=\bar{R}^{\alpha} \triangleright \otimes \bar{R}_{\alpha} \triangleright Q
$$

Use of the braiding map $\tau_{\mathcal{R}}$ (cf. (5.8a)) allows us to rewrite the lift id $\otimes_{\mathcal{R}} Q$ acting on $V \otimes W$ in terms of the lift $Q \otimes$ id acting on $W \otimes V$,

$$
\begin{aligned}
\mathrm{id} \otimes_{\mathcal{R}} Q & =\bar{R}^{\alpha} \triangleright \otimes \bar{R}_{\alpha} \triangleright Q \\
& =\bar{R}^{\alpha} \triangleright \otimes\left(\bar{R}_{\alpha_{1}} \triangleright \circ Q \circ S\left(\bar{R}_{\alpha_{2}}\right) \triangleright\right) \\
& =\bar{R}^{\alpha} \bar{R}^{\beta} \triangleright \otimes\left(\bar{R}_{\alpha} \triangleright \circ Q \circ S\left(\bar{R}_{\beta}\right) \triangleright\right) \\
& =\bar{R}^{\alpha} R^{\beta} \triangleright \otimes\left(\bar{R}_{\alpha} \triangleright \circ Q \circ R_{\beta} \triangleright\right) \\
& =\tau_{\mathcal{R}} \circ(Q \otimes \mathrm{id}) \circ \tau_{\mathcal{R}}^{-1} .
\end{aligned}
$$

We now show that the $\mathcal{R}$-tensor product $\otimes_{\mathcal{R}}$ is compatible with the ${ }^{H} \mathscr{M}$-module structure, that it is associative and that it satisfies a braided composition law.

Theorem 5.4. Let $(H, \mathcal{R})$ be a quasitriangular Hopf algebra and $V, W, Z$, $\widetilde{V}, \widetilde{W}, \widetilde{Z}, \widehat{V}, \widehat{W}$ be ${ }^{H} \mathscr{M}$-modules. The $\mathcal{R}$-tensor product is compatible with the ${ }^{H} \mathscr{M}$-module structure, i.e., for all $\xi \in H, P \in \operatorname{Hom}_{\mathbb{K}}(V, \widetilde{V})$ and $Q \in$ $\operatorname{Hom}_{\mathbb{K}}(W, \widetilde{W})$,

$$
\xi \triangleright\left(P \otimes_{\mathcal{R}} Q\right)=\left(\xi_{1} \triangleright P\right) \otimes_{\mathcal{R}}\left(\xi_{2} \triangleright Q\right) .
$$

Furthermore, the $\mathcal{R}$-tensor product is associative, i.e., for all $P \in \operatorname{Hom}_{\mathbb{K}}(V$, $\widetilde{V}), Q \in \operatorname{Hom}_{\mathbb{K}}(W, \widetilde{W})$ and $T \in \operatorname{Hom}_{\mathbb{K}}(Z, \widetilde{Z})$,

$$
\left(P \otimes_{\mathcal{R}} Q\right) \otimes_{\mathcal{R}} T=P \otimes_{\mathcal{R}}\left(Q \otimes_{\mathcal{R}} T\right),
$$


and satisfies the braided composition law, for all $P \in \operatorname{Hom}_{\mathbb{K}}(V, \widetilde{V}), Q \in$ $\operatorname{Hom}_{\mathbb{K}}(W, \widetilde{W}), \widetilde{P} \in \operatorname{Hom}_{\mathbb{K}}(\widetilde{V}, \widehat{V})$ and $\widetilde{Q} \in \operatorname{Hom}_{\mathbb{K}}(\widetilde{W}, \widehat{W})$,

$$
\left(\widetilde{P} \otimes_{\mathcal{R}} \widetilde{Q}\right) \circ\left(P \otimes_{\mathcal{R}} Q\right)=\left(\widetilde{P} \circ\left(\bar{R}^{\alpha} \triangleright P\right)\right) \otimes_{\mathcal{R}}\left(\left(\bar{R}_{\alpha} \triangleright \widetilde{Q}\right) \circ Q\right)
$$

Proof. From (5.13) we have compatibility between the $H$-action and the lift $P \mapsto P \otimes$ id, for all $\xi \in H$,

$$
\xi \triangleright(P \otimes \mathrm{id})=(\xi \triangleright P) \otimes \mathrm{id} .
$$

Compatibility between the $H$-action and the lift $Q \mapsto \operatorname{id} \otimes_{\mathcal{R}} Q$ follows from (5.9) and (5.19),

$$
\begin{aligned}
\xi \triangleright\left(\mathrm{id} \otimes_{\mathcal{R}} Q\right) & =\xi \triangleright\left(\tau_{\mathcal{R}} \circ(Q \otimes \mathrm{id}) \circ \tau_{\mathcal{R}}^{-1}\right) \\
& =\tau_{\mathcal{R}} \circ \xi \triangleright(Q \otimes \mathrm{id}) \circ \tau_{\mathcal{R}}^{-1} \\
& =\tau_{\mathcal{R}} \circ((\xi \triangleright Q) \otimes \mathrm{id}) \circ \tau_{\mathcal{R}}^{-1} \\
& =\mathrm{id} \otimes_{\mathcal{R}} \xi \triangleright Q .
\end{aligned}
$$

Equation (5.18a) then follows from $P \otimes_{\mathcal{R}} Q=\left(P \otimes_{\mathcal{R}}\right.$ id $) \circ\left(\mathrm{id} \otimes_{\mathcal{R}} Q\right)$ and the left $H$-action property $\xi \triangleright(T \circ \check{T})=\xi_{1} \triangleright T \circ \xi_{2} \triangleright \check{T}$, that holds for any two composable $\mathbb{K}$-linear maps $T$ and $\check{T}$.

We now prove $(5.18 \mathrm{~b})$. The left hand side of $(5.18 \mathrm{~b})$ can be expanded as follows

$$
\begin{aligned}
\left(P \otimes_{\mathcal{R}} Q\right) \otimes_{\mathcal{R}} T & =\left(\left(P \otimes_{\mathcal{R}} Q\right) \circ\left(\bar{R}_{1}^{\alpha} \triangleright \otimes \bar{R}_{2}^{\alpha} \triangleright\right)\right) \otimes\left(\bar{R}_{\alpha} \triangleright T\right) \\
& =\left(P \circ \bar{R}^{\beta} \bar{R}_{1}^{\alpha} \triangleright\right) \otimes\left(\left(\bar{R}_{\beta} \triangleright Q\right) \circ \bar{R}_{2}^{\alpha} \triangleright\right) \otimes\left(\bar{R}_{\alpha} \triangleright T\right) \\
(5.21 \mathrm{a}) \quad & \left(P \circ \bar{R}^{\beta} \bar{R}^{\alpha} \triangleright\right) \otimes\left(\left(\bar{R}_{\beta} \triangleright Q\right) \circ \bar{R}^{\gamma} \triangleright\right) \otimes\left(\bar{R}_{\gamma} \bar{R}_{\alpha} \triangleright T\right),
\end{aligned}
$$

where in the third line we have used (5.5b). This expression equals the right hand side of $(5.18 \mathrm{~b})$, indeed

$$
\begin{aligned}
P \otimes_{\mathcal{R}}\left(Q \otimes_{\mathcal{R}} T\right) & =\left(P \circ \bar{R}^{\alpha} \triangleright\right) \otimes\left(\bar{R}_{\alpha_{1}} \triangleright Q \otimes_{\mathcal{R}} \bar{R}_{\alpha_{2}} \triangleright T\right) \\
& =\left(P \circ \bar{R}^{\alpha} \triangleright\right) \otimes\left(\left(\bar{R}_{\alpha_{1}} \triangleright Q\right) \circ \bar{R}^{\gamma} \triangleright\right) \otimes\left(\bar{R}_{\gamma} \bar{R}_{\alpha_{2}} \triangleright T\right) \\
(5.21 \mathrm{~b}) \quad & \left(P \circ \bar{R}^{\beta} \bar{R}^{\alpha} \triangleright\right) \otimes\left(\left(\bar{R}_{\beta} \triangleright Q\right) \circ \bar{R}^{\gamma} \triangleright\right) \otimes\left(\bar{R}_{\gamma} \bar{R}_{\alpha} \triangleright T\right),
\end{aligned}
$$

where in the third line we have used (5.5c). 
Finally, we show (5.18c). From (5.15) we have

$$
\left(\widetilde{P} \otimes_{\mathcal{R}} \widetilde{Q}\right) \circ\left(P \otimes_{\mathcal{R}} Q\right)=\left(\widetilde{P} \otimes_{\mathcal{R}} \text { id }\right) \circ\left(\mathrm{id} \otimes_{\mathcal{R}} \widetilde{Q}\right) \circ\left(P \otimes_{\mathcal{R}} \text { id }\right) \circ\left(\operatorname{id} \otimes_{\mathcal{R}} Q\right)
$$

and therefore $(5.18 \mathrm{c})$ is proven if

$$
\left(\mathrm{id} \otimes_{\mathcal{R}} \widetilde{Q}\right) \circ\left(P \otimes_{\mathcal{R}} \mathrm{id}\right)=\bar{R}^{\alpha} \triangleright\left(P \otimes_{\mathcal{R}} \mathrm{id}\right) \circ \bar{R}_{\alpha} \triangleright\left(\mathrm{id} \otimes_{\mathcal{R}} \widetilde{Q}\right)
$$

or equivalently $\left(\mathrm{id} \otimes_{\mathcal{R}} \widetilde{Q}\right) \circ\left(P \otimes_{\mathcal{R}} \mathrm{id}\right)=\left(\bar{R}^{\alpha} \triangleright P \otimes_{\mathcal{R}} \mathrm{id}\right) \circ\left(\mathrm{id} \otimes_{\mathcal{R}} \bar{R}_{\alpha} \triangleright \widetilde{Q}\right)$. This last equality holds true because (use (5.16) and (5.5b)),

$$
\begin{aligned}
\left(\mathrm{id} \otimes_{\mathcal{R}} \widetilde{Q}\right) \circ\left(P \otimes_{\mathcal{R}} \mathrm{id}\right) & =\left(\bar{R}^{\alpha} \triangleright \otimes \bar{R}_{\alpha} \triangleright \widetilde{Q}\right) \circ(P \otimes \mathrm{id}) \\
& =\left(\bar{R}^{\alpha} \triangleright \circ\right) \otimes\left(\bar{R}_{\alpha} \triangleright \widetilde{Q}\right) \\
& =\left(\left(\bar{R}_{1}^{\alpha} \triangleright P\right) \circ \bar{R}_{2}^{\alpha} \triangleright\right) \otimes\left(\bar{R}_{\alpha} \triangleright \widetilde{Q}\right) \\
& =\left(\left(\bar{R}^{\alpha} \triangleright P\right) \circ \bar{R}^{\beta} \triangleright\right) \otimes\left(\bar{R}_{\beta} \bar{R}_{\alpha} \triangleright \widetilde{Q}\right) \\
& =\left(\bar{R}^{\alpha} \triangleright P\right) \otimes_{\mathcal{R}}\left(\bar{R}_{\alpha} \triangleright \widetilde{Q}\right) .
\end{aligned}
$$

Let us now consider the case where $V, \widetilde{V}$ are ${ }^{H} \mathscr{M}$-modules and $W, \widetilde{W}$

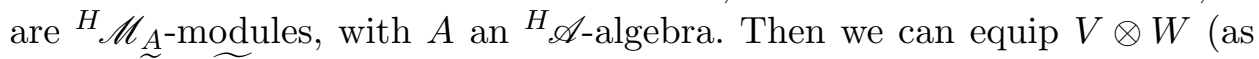
well as $\widetilde{V} \otimes \widetilde{W})$ with a right $A$-module structure by defining $(v \otimes w) \cdot a:=$ $v \otimes(w \cdot a)$, for all $v \in V, w \in W$ and $a \in A$. This right $A$-action is extended by $\mathbb{K}$-linearity to all $V \otimes W$. Moreover we have that $V \otimes W$ and $\widetilde{V} \otimes \widetilde{W}$ are ${ }^{H} \mathscr{M}_{A}$-modules, where the left $H$-action is given in (5.1).

Proposition 5.5. Let $(H, \mathcal{R})$ be a quasitriangular Hopf algebra, $A$ be an ${ }^{H} \mathscr{A}$-algebra, $V, \widetilde{V}$ be two ${ }^{H} \mathscr{M}$-modules and $W, \widetilde{W}$ be two ${ }^{H} \mathscr{M}_{A}$-modules. Then we have, for all $P \in \operatorname{Hom}_{\mathbb{K}}(V, \widetilde{V})$ and $Q \in \operatorname{Hom}_{A}(W, \widetilde{W})$,

$$
P \otimes_{\mathcal{R}} Q \in \operatorname{Hom}_{A}(V \otimes W, \widetilde{V} \otimes \widetilde{W}) .
$$

Proof. For all $a \in A, v \in V$ and $w \in W$,

$$
\begin{aligned}
\left(P \otimes_{\mathcal{R}} Q\right)((v \otimes w) \cdot a) & =\left(P \otimes_{\mathcal{R}} Q\right)(v \otimes(w \cdot a)) \\
& =P\left(\bar{R}^{\alpha} \triangleright v\right) \otimes\left(\bar{R}_{\alpha} \triangleright Q\right)(w \cdot a) \\
& =P\left(\bar{R}^{\alpha} \triangleright v\right) \otimes\left(\bar{R}_{\alpha} \triangleright Q\right)(w) \cdot a \\
& =\left(\left(P \otimes_{\mathcal{R}} Q\right)(v \otimes w)\right) \cdot a,
\end{aligned}
$$


where in the second line we have used that $\xi \triangleright Q \in \operatorname{Hom}_{A}(W, \widetilde{W})$, for all $\xi \in H$.

\subsection{Deformation}

We study the twist deformation of tensor products of ${ }^{H} \mathscr{M}$-modules and $\mathbb{K}$-linear maps.

Since as algebras $H$ and $H^{\mathcal{F}}$ coincide, we have that any ${ }^{H} \mathscr{M}$-module $V$ is equivalently an ${ }^{H^{\mathcal{F}}} \mathscr{M}$-module. It is however convenient to distinguish between these two module structures and hence we denote by $V_{\star}$ the $\mathbb{K}$ module $V$ with the ${ }^{H^{\mathcal{F}}} \mathscr{M}$-module structure. This notation agrees with that of Theorem 3.5 where we deformed an ${ }^{H}{ }_{A} \mathscr{M}_{B}$-module $V$ into an ${ }^{H^{\mathcal{F}}}{ }_{A_{\star}} \mathscr{M}_{B_{\star}-}$ module $V_{\star}$ (just consider trivial algebras $A=B=\mathbb{K}$ ).

Given two $H^{\mathcal{F}} \mathscr{M}$-modules $V_{\star}, W_{\star}$ we denote their tensor product by $V_{\star} \otimes_{\star}$ $W_{\star}$. By definition $V_{\star} \otimes_{\star} W_{\star}$ equals $V \otimes W$ as $\mathbb{K}$-module; the ${ }^{H^{\mathcal{F}}} \mathscr{M}$-module structure is canonically given by the $H^{\mathcal{F}}$-coproduct (cf. (5.1)), for all $\xi \in$ $H^{\mathcal{F}}, v \in V_{\star}$ and $w \in W_{\star}, \xi \triangleright_{\mathcal{F}}\left(v \otimes_{\star} w\right):=\xi_{1_{\mathcal{F}}} \triangleright v \otimes_{\star} \xi_{2_{\mathcal{F}}} \triangleright w$ (and extended by $\mathbb{K}$-linearity to all elements in $\left.V_{\star} \otimes_{\star} W_{\star}\right)$.

We now compare the $H^{\mathcal{F}} \mathscr{M}$-modules $V_{\star} \otimes_{\star} W_{\star}$ and $(V \otimes W)_{\star}$; in this latter the $H^{\mathcal{F}}$-action is just the $H$-action on $V \otimes W$, hence it is obtained using the $H$-coproduct.

It is easy to show that the $\mathbb{K}$-linear map

$$
\varphi_{V, W}:=\mathcal{F}^{-1} \triangleright: V_{\star} \otimes_{\star} W_{\star} \rightarrow(V \otimes W)_{\star},
$$

$$
v \otimes_{\star} w \mapsto \varphi_{V, W}\left(v \otimes_{\star} w\right)=\left(\overline{\mathrm{f}}^{\alpha} \triangleright v\right) \otimes\left(\overline{\mathrm{f}}_{\alpha} \triangleright w\right)
$$

provides an isomorphism between the ${ }^{H^{\mathcal{F}}} \mathscr{M}$-modules $V_{\star} \otimes_{\star} W_{\star}$ and $(V \otimes$ $W)_{\star}$. Indeed it intertwines between the two $H^{\mathcal{F}}$-actions,

$$
\begin{aligned}
\varphi_{V, W}\left(\xi \triangleright_{\mathcal{F}}\left(v \otimes_{\star} w\right)\right) & =\varphi_{V, W}\left(\left(\xi_{1_{\mathcal{F}}} \triangleright v\right) \otimes_{\star}\left(\xi_{2_{\mathcal{F}}} \triangleright w\right)\right) \\
& =\left(\xi_{1} \overline{\mathrm{f}}^{\alpha} \triangleright v\right) \otimes\left(\xi_{2} \overline{\mathrm{f}}_{\alpha} \triangleright w\right) \\
& =\xi \triangleright \varphi_{V, W}\left(v \otimes_{\star} w\right) .
\end{aligned}
$$

The inverse of $\varphi_{V, W}$ is

$$
\begin{aligned}
\varphi_{V, W}^{-1}:=\mathcal{F} \triangleright:(V \otimes W)_{\star} & \rightarrow V_{\star} \otimes_{\star} W_{\star}, \\
v \otimes w & \mapsto \varphi_{V, W}^{-1}(v \otimes w)=\left(\mathrm{f}^{\alpha} \triangleright v\right) \otimes_{\star}\left(\mathrm{f}_{\alpha} \triangleright w\right) .
\end{aligned}
$$

Consider now three ${ }^{H} \mathscr{M}$-modules $V, W, \Omega$. The twist cocycle property (3.4c) immediately implies the following commutative diagram of $H^{\mathcal{F}} \mathscr{M}$-module 
isomorphisms

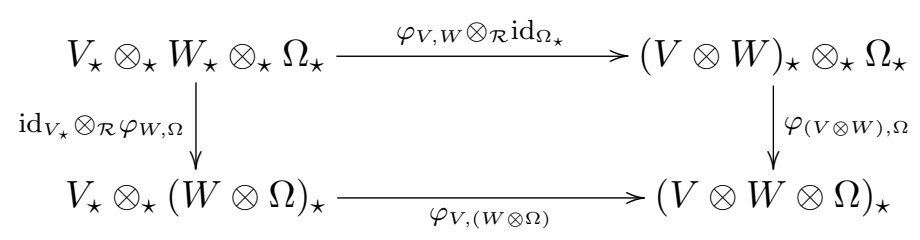

Concerning the tensor product of $\mathbb{K}$-linear maps, on one hand the deformation of $P \otimes_{\mathcal{R}} Q \in H_{\mathbb{K}}(V \otimes W, \widetilde{V} \otimes \widetilde{W})$, according to Theorem 4.7, is given by $D_{\mathcal{F}}\left(P \otimes_{\mathcal{R}} Q\right) \in \operatorname{Hom}_{\mathbb{K}}\left((V \otimes W)_{\star},(\widetilde{V} \otimes \widetilde{W})_{\star}\right)$. On the other hand, we recall that if $(H, \mathcal{R})$ is a quasitriangular Hopf algebra with twist $\mathcal{F} \in$ $H \otimes H$, then $\left(H^{\mathcal{F}}, \mathcal{R}^{\mathcal{F}}=\mathcal{F}_{21} \mathcal{R} \mathcal{F}^{-1}\right)$ is a quasitriangular Hopf algebra. We therefore have the tensor product $\otimes_{\mathcal{R}^{\mathcal{F}}}$ of $\mathbb{K}$-linear maps between $H^{\mathcal{F}} \mathscr{M}$ modules. In particular, the lift of a $\mathbb{K}$-linear map $P_{\star}: V_{\star} \rightarrow \widetilde{V}_{\star}$ to the ${ }^{H^{\mathcal{F}}} \mathscr{M}$ module $V_{\star} \otimes_{\star} W_{\star}$ is the $\mathbb{K}$-linear map $P_{\star} \otimes_{\mathcal{R}^{\mathcal{F}}}$ id $:=P_{\star} \otimes_{\star}$ id that as usual is defined by, for all $v \in V_{\star}, w \in W_{\star}$,

$$
\left(P_{\star} \otimes_{\mathcal{R}^{\mathcal{F}}} \mathrm{id}\right)\left(v \otimes_{\star} w\right):=\left(P_{\star} \otimes_{\star} \mathrm{id}\right)\left(v \otimes_{\star} w\right):=P_{\star}(v) \otimes_{\star} w
$$

Theorem 5.6. Let $(H, \mathcal{R})$ be a quasitriangular Hopf algebra with twist $\mathcal{F} \in$ $H \otimes H$ and $V, W, \widetilde{V}, \widetilde{W}$ be ${ }^{H} \mathscr{M}$-modules. Then for all $P \in H_{\mathbb{K}}(V, \widetilde{V})$ and $Q \in \operatorname{Hom}_{\mathbb{K}}(W, \widetilde{W})$ the following diagram of $\mathbb{K}$-linear maps commutes:

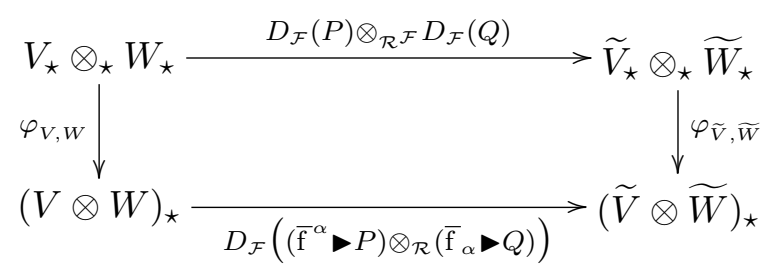

i.e.

$$
D_{\mathcal{F}}\left(\left(\overline{\mathrm{f}}^{\alpha} \triangleright P\right) \otimes_{\mathcal{R}}\left(\overline{\mathrm{f}}_{\alpha} \triangleright Q\right)\right)=\varphi_{\widetilde{V}, \widetilde{W}} \circ\left(D_{\mathcal{F}}(P) \otimes_{\mathcal{R}^{\mathcal{F}}} D_{\mathcal{F}}(Q)\right) \circ \varphi_{V, W}^{-1} .
$$

Proof. Use of (5.15) and compatibility between the $H$-action and the lifts of $P$ and $Q$ (cf. (5.19) and (5.20)) shows that (5.33) is equivalent to

$$
D_{\mathcal{F}}\left(\left(P \otimes_{\mathcal{R}} \mathrm{id}\right) \circ_{\star}\left(\mathrm{id} \otimes_{\mathcal{R}} Q\right)\right)
$$

$$
=\varphi_{\widetilde{V}, \widetilde{W}} \circ\left(D_{\mathcal{F}}(P) \otimes_{\mathcal{R}^{\mathcal{F}}} \mathrm{id}\right) \circ\left(\mathrm{id} \otimes_{\mathcal{R}^{\mathcal{F}}} D_{\mathcal{F}}(Q)\right) \circ \varphi_{V, W}^{-1} .
$$


Because of the algebra isomorphism (3.26), see also Theorem 3.16, the thesis (5.33) is equivalent to

$$
D_{\mathcal{F}}\left(P \otimes_{\mathcal{R}} \text { id }\right) \circ D_{\mathcal{F}}\left(\mathrm{id} \otimes_{\mathcal{R}} Q\right)
$$

$$
=\varphi_{\widetilde{V}, \widetilde{W}} \circ\left(D_{\mathcal{F}}(P) \otimes_{\mathcal{R}^{\mathcal{F}}} \mathrm{id}\right) \circ\left(\mathrm{id} \otimes_{\mathcal{R}^{\mathcal{F}}} D_{\mathcal{F}}(Q)\right) \circ \varphi_{V, W}^{-1} .
$$

The deformation of $P \otimes_{\mathcal{R}}$ id $=P \otimes \mathrm{id}: V \otimes \widetilde{W} \rightarrow \widetilde{V} \otimes \widetilde{W}$ (and also of $Q \otimes$ id) can be simplified as follows

$$
\begin{aligned}
D_{\mathcal{F}}(P \otimes \mathrm{id}) & =\left(\left(\overline{\mathrm{f}}^{\alpha} \triangleright P\right) \circ \overline{\mathrm{f}}_{\left.\alpha_{1} \triangleright\right) \otimes \overline{\mathrm{f}}_{\alpha_{2}} \triangleright}\right. \\
& =\left(\left(\overline{\mathrm{f}}_{1}^{\alpha} \overline{\mathrm{f}}^{\beta}-P\right) \circ \overline{\mathrm{f}}_{2}^{\alpha} \overline{\mathrm{f}}_{\beta} \mathrm{f}_{\triangleright} \gamma_{\triangleright}\right) \otimes \overline{\mathrm{f}}_{\alpha} \mathrm{f}_{\gamma} \triangleright \\
& =\left(\overline{\mathrm{f}}^{\alpha} \triangleright \circ D_{\mathcal{F}}(P) \circ \mathrm{f}_{\triangleright}{ }_{\triangleright}\right) \otimes \overline{\mathrm{f}}_{\alpha} \mathrm{f}_{\gamma} \triangleright \\
& =\varphi_{\widetilde{V}, \widetilde{W}} \circ\left(D_{\mathcal{F}}(P) \otimes_{\star} \mathrm{id}\right) \circ \varphi_{V, \widetilde{W}}^{-1},
\end{aligned}
$$

where in the second line we inserted on the right id $\otimes \mathrm{id}=\mathcal{F}^{-1} \mathcal{F} \triangleright$ and then used the twist cocycle property (3.4c). In the last line we used (5.31).

The deformation of

$$
\operatorname{id} \otimes_{\mathcal{R}} Q=\tau_{\mathcal{R}} \circ(Q \otimes \mathrm{id}) \circ \tau_{\mathcal{R}}^{-1}=\tau_{\mathcal{R}} \circ_{\star}(Q \otimes \mathrm{id}) \circ_{\star} \tau_{\mathcal{R}}^{-1}
$$

(where in the last equality we used that $\tau_{\mathcal{R}}$ and $\tau_{\mathcal{R}}^{-1}$ are $H$-equivariant, i.e. $\xi \triangleright \tau_{\mathcal{R}}=\varepsilon(\xi) \tau_{\mathcal{R}}$ and $\xi \triangleright \tau_{\mathcal{R}}^{-1}=\varepsilon(\xi) \tau_{\mathcal{R}}^{-1}$, for all $\left.\xi \in H\right)$ is given by

$$
\begin{aligned}
D_{\mathcal{F}}\left(\mathrm{id} \otimes_{\mathcal{R}} Q\right) & =\tau_{\mathcal{R}} \circ \varphi_{\widetilde{W}, V} \circ\left(D_{\mathcal{F}}(Q) \otimes_{\star} \mathrm{id}\right) \circ \varphi_{W, V}^{-1} \circ \tau_{\mathcal{R}}^{-1} \\
& =\varphi_{V, \widetilde{W}} \circ \tau_{\mathcal{R}^{\mathcal{F}}} \circ\left(D_{\mathcal{F}}(Q) \otimes_{\star} \mathrm{id}\right) \circ \tau_{\mathcal{R}^{\mathcal{F}}}^{-1} \circ \varphi_{V, W}^{-1},
\end{aligned}
$$

where in the first equality we again used that $\tau_{\mathcal{R}}$ is $H$-equivariant. In the last equality we have defined $\tau_{\mathcal{R}^{\mathcal{F}}}: \widetilde{W}_{\star} \otimes_{\star} V_{\star} \rightarrow V_{\star} \otimes_{\star} \widetilde{W}_{\star}$ by $\tau_{\mathcal{R}^{\mathcal{F}}}:=\varphi_{V, \widetilde{W}}^{-1} \circ$ $\tau_{\mathcal{R}} \circ \varphi_{\widetilde{W}, V}$ and $\tau_{\mathcal{R}^{\mathcal{F}}}^{-1}: V_{\star} \otimes_{\star} W_{\star} \rightarrow W_{\star} \otimes_{\star} V_{\star}$ by $\tau_{\mathcal{R}^{\mathcal{F}}}^{-1}:=\varphi_{W, V}^{-1} \circ \tau_{\mathcal{R}}^{-1} \circ \varphi_{V, W}$.

Equality (5.35) holds because $\tau_{\mathcal{R}^{\mathcal{F}}}: W_{\star} \otimes_{\star} V_{\star} \rightarrow V_{\star} \otimes_{\star} W_{\star}$ defined by $\tau_{\mathcal{R}^{\mathcal{F}}}:=\varphi_{V, W}^{-1} \circ \tau_{\mathcal{R}} \circ \varphi_{W, V}$ is easily seen to be the braiding map for the twist deformed tensor product, for all $v \in V_{\star}, w \in W_{\star}$,

$$
\tau_{\mathcal{R}^{\mathcal{F}}}\left(w \otimes_{\star} v\right)=\left(\bar{R}^{\mathcal{F} \alpha} \triangleright v\right) \otimes_{\star}\left(\bar{R}_{\alpha}^{\mathcal{F}} \triangleright w\right)
$$

so that, as in $(5.17)$, we have, $\tau_{\mathcal{R}^{\mathcal{F}}} \circ\left(D_{\mathcal{F}}(Q) \otimes_{\star} \mathrm{id}\right) \circ \tau_{\mathcal{R}^{\mathcal{F}}}^{-1}=\mathrm{id} \otimes_{\mathcal{R}^{\mathcal{F}}} D_{\mathcal{F}}(Q)$. 
Remark 5.7. We provide a categorical description of the results in Subsections 5.2 and 5.3, and show that, because of commutativity of the diagrams (5.30) and (5.32), the equivalence of the categories $\operatorname{rep}^{H} \star$ and $\operatorname{rep}^{H^{\mathcal{F}}}$ proven after Theorem 3.16 extends to the tensor product structures that can be considered on these categories.

We recall that an object in rep $^{H}$ is an ${ }^{H} \mathscr{M}$-module $V$ and a morphism between two objects $V, W$ in $\operatorname{rep}^{H}$ is a $\mathbb{K}$-linear map $P \in \operatorname{Hom}_{\mathbb{K}}(V, W)$ (not necessarily $H$-equivariant). Let us consider the association $\otimes_{\mathcal{R}}: \operatorname{rep}^{H} \times$ rep $^{H} \rightarrow$ rep $^{H}$ given on objects $(V, W)$ by the tensor product $V \otimes W$ and on morphisms $(P, Q)$ by the $\mathcal{R}$-tensor product of $\mathbb{K}$-linear maps $P \otimes_{\mathcal{R}} Q$ (cf. Definition 5.3). Because of the braided composition law (5.18c) this is not a bifunctor. We say that it is "almost" a bifunctor and refer to $\left(\operatorname{rep}^{H}, \otimes_{\mathcal{R}}\right)$ as an "almost monoidal" category.

We similarly have the "almost monoidal" category $\left(\operatorname{rep}^{H^{\mathcal{F}}}, \otimes_{\mathcal{R}^{\mathcal{F}}}\right)$, where the association $\otimes_{\mathcal{R}^{\mathcal{F}}}: \operatorname{rep}^{H^{\mathcal{F}}} \times \operatorname{rep}^{H^{\mathcal{F}}} \rightarrow \operatorname{rep}^{H^{\mathcal{F}}}$ is defined on objects $\left(V_{\star}, W_{\star}\right)$ by $V_{\star} \otimes_{\star} W_{\star}$ and on morphisms $\left(P_{\star}, Q_{\star}\right)$ by $P_{\star} \otimes_{\mathcal{R}^{\mathcal{F}}} Q_{\star}$. Notice that $\mathbb{K}$-linear maps between $H^{\mathcal{F}} \mathscr{M}$-modules are denoted with a $\star$-index, like $P_{\star}: V_{\star} \rightarrow W_{\star}$, because they carry the $H^{\mathcal{F}}$-adjoint action, rather than the $H$-adjoint action carried by $\mathbb{K}$-linear maps between ${ }^{H} \mathscr{M}$-modules $P: V \rightarrow W$. This adjoint action enters the definition of the tensor product morphism $P_{\star} \otimes_{\mathcal{R}^{\mathcal{F}}} Q_{\star}$.

We also consider the "almost monoidal" category $\left(\operatorname{rep}_{\star}^{H}, \otimes_{\mathcal{R}_{\star}}\right)$, where the association $\otimes_{\mathcal{R} \star}: \operatorname{rep}_{\star}^{H} \times \operatorname{rep}_{\star}^{H} \rightarrow \operatorname{rep}_{\star}^{H}$ is defined on objects $(V, W)$ by $V \otimes W$ and on morphisms $(P, Q)$ by $P \otimes_{\mathcal{R} \star} Q=\left(\overline{\mathrm{f}}^{\alpha} \rightarrow P\right) \otimes_{\mathcal{R}}\left(\overline{\mathrm{f}}_{\alpha} \rightarrow Q\right)$ (the braided composition law reads as (5.18c) with composition of morphisms given by the $\star$-composition $o_{\star}$, and tensor product of morphisms given by $\left.\otimes_{\mathcal{R}_{\star}}\right)$.

The equivalence of the categories rep $^{H} \star$ and rep $^{H^{\mathcal{F}}}$ shown after Theorem 3.16 extends to an equivalence of $\left(\operatorname{rep}^{H}, \otimes_{\mathcal{R}_{\star}}\right)$ and $\left(\operatorname{rep}^{H^{\mathcal{F}}}, \otimes_{\mathcal{R}^{\mathcal{F}}}\right)$ as "almost monoidal" categories: the collection of maps $\varphi_{V, W}: V_{\star} \otimes_{\star} W_{\star} \rightarrow$ $(V \otimes W)_{\star}$ provides a natural isomorphism between the "almost bifunctors" $\otimes_{R^{\mathcal{F}}}$ and $\otimes_{\mathcal{R}_{\star}}$. This is so because the $\varphi_{V, W}$ maps satisfy the commutative diagrams (5.30) and (5.32).

Notice that when we restrict ourselves to $H$-equivariant morphisms, the $\mathcal{R}$-tensor product reduces to the usual tensor product (5.11) and the "almost monoidal" category $\left(\operatorname{rep}^{H}, \otimes_{\mathcal{R}}\right)$ restricts to the monoidal category $\left(\operatorname{rep}_{\text {eqv }}^{H}, \otimes\right)$. In this case we recover the results of Drinfeld [14] on the equivalence of the monoidal categories $\left(\operatorname{rep}_{\text {eqv }}^{H}, \otimes\right)$ and $\left(\operatorname{rep}_{\text {eqv }}^{H^{\mathcal{F}}}, \otimes_{\star}\right)$. 


\subsection{Quasi-commutative algebras and bimodules} (tensor product over $A$ )

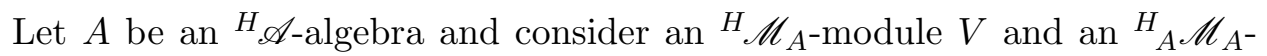
module $W$. We have that $V \otimes W$ is an ${ }^{H} \mathscr{M}_{A}$-module, where the module structure is given by, for all $\xi \in H, v \in V, w \in W, a \in A, \xi \triangleright(v \otimes w)=\left(\xi_{1} \triangleright\right.$ $v) \otimes\left(\xi_{2} \triangleright w\right)$ and $(v \otimes w) \cdot a=v \otimes(w \cdot a)$.

We now consider $V \otimes_{A} W$, i.e., the tensor product over $A$ of $V$ and $W$. We recall that it can be defined as the quotient of the $\mathbb{K}$-module $V \otimes W$ via the $\mathbb{K}$-submodule $\mathcal{N}_{V, W}$ generated by the elements $v \cdot a \otimes w-v \otimes a \cdot w$, for all $a \in A, v \in V, w \in W$. The image of $v \otimes w$ under the canonical projection $\pi: V \otimes W \rightarrow V \otimes_{A} W$ is denoted by $v \otimes_{A} w$. Since the $\mathbb{K}$-submodule

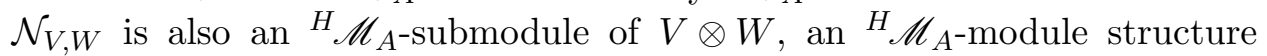
on $V \otimes_{A} W$ is canonically induced from the one on $V \otimes W$. Explicitly we have, for all $\xi \in H, v \in V, w \in W, a \in A, \xi \triangleright\left(v \otimes_{A} w\right)=\left(\xi_{1} \triangleright v\right) \otimes_{A}\left(\xi_{2} \triangleright w\right)$ and $\left(v \otimes_{A} w\right) \cdot a=v \otimes_{A}(w \cdot a)$.

Given two right $A$-linear maps $P \in \operatorname{Hom}_{A}(V, \widetilde{V})$ and $Q \in \operatorname{Hom}_{A}(W, \widetilde{W})$, where $V, \widetilde{V}$ are two ${ }^{H} \mathscr{M}_{A}$-modules and $W, \widetilde{W}$ are two ${ }^{H}{ }_{A} \mathscr{M}_{A}$-modules, following Proposition 5.5 we can construct the $\mathcal{R}$-tensor product $P \otimes_{\mathcal{R}} Q \in$

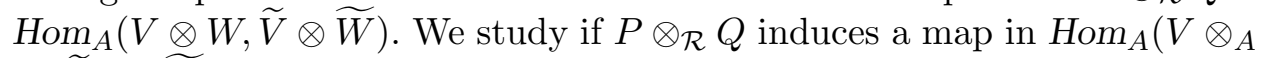
$\left.W, \widetilde{V} \otimes_{A} \widetilde{W}\right)$. In other words, we study if the $\mathcal{R}$-tensor product of $\mathbb{K}$-linear maps induces an $\mathcal{R}$-tensor product between right $A$-linear maps that is compatible with the tensor product of modules over $A$. This requires a quasicommutative structure on ${ }^{H}{ }_{A} \mathscr{M}_{A}$-modules.

Definition 5.8. Let $(H, \mathcal{R})$ be a quasitriangular Hopf algebra. Then an $H_{\mathscr{A}}$-algebra $A$ is called quasi-commutative if, for all $a, \tilde{a} \in A$,

$$
a \tilde{a}=\left(\bar{R}^{\alpha} \triangleright \tilde{a}\right)\left(\bar{R}_{\alpha} \triangleright a\right)
$$

In this case, an ${ }^{H} \mathscr{M}_{A}$-module $V$ is called quasi-commutative if, for all $a \in A, v \in V$,

$$
v \cdot a=\left(\bar{R}^{\alpha} \triangleright a\right) \cdot\left(\bar{R}_{\alpha} \triangleright v\right)
$$

It is not difficult to prove that every ${ }^{H}{ }_{A} \mathscr{M}$-module $V$, with $A$ being a quasi-commutative ${ }^{H} \mathscr{A}$-algebra, is a quasi-commutative ${ }^{H}{ }_{A} \mathscr{M}_{A}$-module with the right $A$-action defined by (5.41).

The quasi-commutativity conditions (5.40) and (5.41) are equivalent to

$$
\tilde{a} a=\left(R_{\alpha} \triangleright a\right)\left(R^{\alpha} \triangleright \tilde{a}\right), a \cdot v=\left(R_{\alpha} \triangleright v\right) \cdot\left(R^{\alpha} \triangleright a\right) .
$$


In the algebra case we can therefore use both $\mathcal{R}^{-1}$ and $\mathcal{R}_{21}$ (being the inverses of the two quasitriangular structures $\mathcal{R}$ and $\mathcal{R}_{21}^{-1}$ on $H$ ) to commute products of algebra elements. This suggests the following definition for the case of modules.

Definition 5.9. Let $(H, \mathcal{R})$ be a quasitriangular Hopf algebra, $A$ a quasicommutative ${ }^{H} \mathscr{A}$-algebra and $V$ a quasi-commutative ${ }^{H}{ }_{A} \mathscr{M}_{A}$-module. We say that $V$ is strong quasi-commutative if in addition, for all $a \in A, v$ $\in V$,

$$
v \cdot a=\left(R_{\alpha} \triangleright a\right) \cdot\left(R^{\alpha} \triangleright v\right)
$$

Equivalently, $V$ is strong quasi-commutative if it is quasi-commutative with respect to both quasitriangular structures $\mathcal{R}$ and $\mathcal{R}_{21}^{-1}$ on $H$.

Example 5.10. The universal enveloping algebra $U \mathfrak{g}$ of a Lie algebra $\mathfrak{g}$ is a cocommutative Hopf algebra. Every cocommutative Hopf algebra $H$ has a triangular structure given by the $\mathcal{R}$-matrix $\mathcal{R}=1 \otimes 1$. Let $\mathcal{F}$ be a twist of this cocommutative Hopf algebra $H$, then the Hopf algebra $H^{\mathcal{F}}$ is triangular with $\mathcal{R}$-matrix $\mathcal{R}^{\mathcal{F}}=\mathcal{F}_{21} \mathcal{F}^{-1}$.

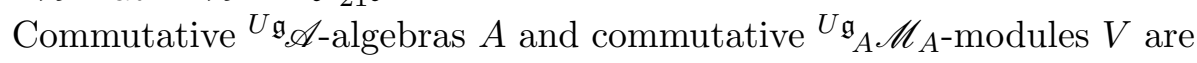
(strong) quasi-commutative, and so are their twist deformations. In particular, the modules and algebras of noncommutative gravity [6] are (strong) quasi-commutative.

Example 5.11. The twist deformation $H^{\mathcal{F}}$ of any quasitriangular (triangular) Hopf algebra $(H, \mathcal{R})$ is quasitriangular (triangular) with $\mathcal{R}$-matrix $\mathcal{R}^{\mathcal{F}}=\mathcal{F}_{21} \mathcal{R F}^{-1}$.

If $A$ is a quasi-commutative ${ }^{H} \mathscr{A}$-algebra, then $A_{\star}$ is a quasi-commutative $H^{\mathcal{F}} \mathscr{A}$-algebra. If $V$ is a (strong) quasi-commutative ${ }^{H} \mathscr{M}_{A^{-}}$-module, then $V_{\star}$ is a (strong) quasi-commutative ${ }^{H^{\mathcal{F}}}{ }_{A_{\star}} \mathscr{M}_{A_{\star}}$-module.

For a triangular Hopf algebra we have $\mathcal{R}=\mathcal{R}_{21}^{-1}$. Thus, every quasicommutative ${ }^{H} \mathscr{M}_{A}$-module is automatically strong quasi-commutative.

Remark 5.12. We presently do not have a nontrivial example of a proper quasitriangular (i.e. not triangular) Hopf algebra $H$ which has a quasicommutative ${ }^{H} \mathscr{A}$-algebra.

Tensor products over $A$ of (strong) quasi-commutative ${ }_{A}{ }_{A} \mathscr{M}_{A}$-modules are again (strong) quasi-commutative ${ }^{H}{ }_{A} \mathscr{M}_{A}$-modules. 
Proposition 5.13. Let $(H, \mathcal{R})$ be a quasitriangular Hopf algebra, $A$ be a quasi-commutative ${ }^{H} \mathscr{A}$-algebra and $V, W$ be two quasi-commutative ${ }^{H} \mathscr{M}_{A^{-}}$ modules. Then $V \otimes_{A} W$ is a quasi-commutative ${ }^{H} \mathscr{M}_{A}$-module.

If moreover $V$ and $W$ are strong quasi-commutative ${ }^{{ }_{A}} \mathscr{M}_{A}$-modules, then $V \otimes_{A} W$ is also a strong quasi-commutative ${ }^{H} \mathscr{M}_{A}$-module.

Proof. We show quasi-commutativity of $V \otimes_{A} W$. For all $v \in V, w \in W$, $a \in A$,

$$
\begin{aligned}
v \otimes_{A} w \cdot a & =v \otimes_{A}\left(\bar{R}^{\alpha} \triangleright a\right) \cdot\left(\bar{R}_{\alpha} \triangleright w\right)=\left(\bar{R}^{\beta} \bar{R}^{\alpha} \triangleright a\right) \cdot\left(\bar{R}_{\beta} \triangleright v\right) \otimes_{A}\left(\bar{R}_{\alpha} \triangleright w\right) \\
(5.44) \quad & =\left(\bar{R}^{\beta} \triangleright a\right) \cdot\left(\bar{R}_{\beta} \triangleright\left(v \otimes_{A} w\right)\right) .
\end{aligned}
$$

The strong quasi-commutativity of $V \otimes_{A} W$, in case of $V$ and $W$ strong quasi-commutative, is proven by considering the equation above with the alternative $\mathcal{R}$-matrix $\mathcal{R}_{21}^{-1}$.

Proposition 5.14. Let $(H, \mathcal{R})$ be a quasitriangular Hopf algebra, $A$ be a quasi-commutative ${ }^{H} \mathscr{A}$-algebra and $V, W$ be two quasi-commutative ${ }^{{ }^{H}}{ }_{\mathscr{M}}{ }_{A^{-}}$ modules. Then $\operatorname{Hom}_{A}(V, W)$ and $\left({ }_{A} \operatorname{Hom}(V, W)\right)^{o p}$ are quasi-commutative ${ }_{A} \mathscr{M}_{A}$-modules.

If moreover $V$ and $W$ are strong quasi-commutative ${ }_{A}{ }_{A} \mathscr{M}_{A}$-modules, then so are $\operatorname{Hom}_{A}(V, W)$ and $\left({ }_{A} \operatorname{Hom}(V, W)\right)^{o p}$.

Proof. Recall that the $A$-bimodule structure of $\operatorname{Hom}_{A}(V, W)$ is defined by, for all $a \in A, P \in \operatorname{Hom}_{A}(V, W), P \cdot a=P \circ l_{a}$ and $a \cdot P=l_{a} \circ P$. We prove that for $A, V$ and $W$ quasi-commutative

$$
P \cdot a=\left(\bar{R}^{\alpha} \triangleright a\right) \cdot\left(\bar{R}_{\alpha} \triangleright P\right) .
$$

Indeed, for all $v \in V$ we have

$$
\begin{aligned}
(P \cdot a)(v) & =P(a \cdot v)=P\left(\left(R_{\alpha} \triangleright v\right) \cdot\left(R^{\alpha} \triangleright a\right)\right)=P\left(R_{\alpha} \triangleright v\right) \cdot\left(R^{\alpha} \triangleright a\right) \\
& =\left(\bar{R}^{\beta} R^{\alpha} \triangleright a\right) \cdot \bar{R}_{\beta} \triangleright\left(P\left(R_{\alpha} \triangleright v\right)\right) \\
& =\left(\bar{R}^{\beta} \bar{R}^{\alpha} \triangleright a\right) \cdot \bar{R}_{\beta} \triangleright\left(P\left(S\left(\bar{R}_{\alpha}\right) \triangleright v\right)\right) \\
& =\left(\bar{R}^{\alpha} \triangleright a\right) \cdot \bar{R}_{\alpha_{1}} \triangleright\left(P\left(S\left(\bar{R}_{\alpha_{2}}\right) \triangleright v\right)\right) \\
& =\left(\bar{R}^{\alpha} \triangleright a\right) \cdot\left(\bar{R}_{\alpha} \triangleright P\right)(v),
\end{aligned}
$$

where in the second equality we used that $a \cdot v=\left(R_{\alpha} \triangleright v\right) \cdot\left(R^{\alpha} \triangleright a\right)$, which is equivalent to $(5.41)$. Then we have used the $\mathcal{R}$-matrix properties $(5.5 \mathrm{c})$ 
and (5.7b). Quasi-commutativity of the ${ }^{H}{ }_{A} \mathscr{M}_{A}$-module $\left({ }_{A} \operatorname{Hom}(V, W)\right)^{o p}$ is similarly proven.

The strong quasi-commutativity of $\operatorname{Hom}_{A}(V, W)$ and $\left({ }_{A} \operatorname{Hom}(V, W)\right)^{o p}$ in the case of strong quasi-commutative ${ }^{H} \mathscr{M}_{A}$-modules $V$ and $W$ is similarly proven considering the alternative $\mathcal{R}$-matrix $\mathcal{R}_{21}^{-1}$.

Remark 5.15. A particular case is when $V=W$, then $\operatorname{End}_{A}(V)$ is a quasi-commutative ${ }^{H}{ }_{A} \mathscr{M}_{A}$-module. However the ${ }^{H}{ }_{A} \mathscr{A}_{A}$-algebra structure of $\operatorname{End}_{A}(V)$ is in general not quasi-commutative. Similarly $\left({ }_{A} \operatorname{End}(V)\right)^{o p}$ is quasi-commutative as an ${ }^{H} \mathscr{M}_{A^{-}}$module but in general not as an ${ }_{A} \mathscr{A}_{A^{-}}$ algebra.

Theorem 5.16. Let $(H, \mathcal{R})$ be a quasitriangular Hopf algebra, $A$ be a quasi-commutative ${ }^{H} \mathscr{A}$-algebra, $V, \widetilde{V}$ be two ${ }^{H} \mathscr{M}_{A}$-modules and $W, \widetilde{W}$ be two quasi-commutative ${ }_{A} \mathscr{M}_{A}$-modules. For all $P \in \operatorname{Hom}_{A}(V, \widetilde{V})$ and $Q \in$ $\operatorname{Hom}_{A}(W, \widetilde{W})$ the map $P \otimes_{\mathcal{R}} Q \in \operatorname{Hom}_{A}(V \otimes W, \widetilde{V} \otimes \widetilde{W})$ induces a welldefined right A-linear map (denoted by the same symbol) $P \otimes_{\mathcal{R}} Q: V \otimes_{A}$ $W \rightarrow \widetilde{V} \otimes_{A} \widetilde{W}$ on the quotient modules. Explicitly we have, for all $v \in V$ and $w \in W$,

$$
P \otimes_{\mathcal{R}} Q\left(v \otimes_{A} w\right)=P\left(\bar{R}^{\alpha} \triangleright v\right) \otimes_{A}\left(\bar{R}_{\alpha} \triangleright Q\right)(w) .
$$

Proof. Remember that the ${ }^{H} \mathscr{M}_{A}$-module $V \otimes_{A} W$ was defined as the quotient of the ${ }^{H} \mathscr{M}_{A}$-module $V \otimes W$ via the ${ }^{H} \mathscr{M}_{A}$-submodule $\mathcal{N}_{V, W}$ generated by the elements $v \cdot a \otimes w-v \otimes a \cdot w$, for all $a \in A, v \in V, w \in W$. The map $P \otimes_{\mathcal{R}} Q: V \otimes W \rightarrow \widetilde{V} \otimes \widetilde{W}$ induces a well-defined map on the quotient modules $V \otimes_{A} W=V \otimes W / \mathcal{N}_{V, W}$ and $\widetilde{V} \otimes_{A} \widetilde{W}=\widetilde{V} \otimes \widetilde{W} / \mathcal{N}_{\widetilde{V}, \widetilde{W}}$, if the image of $\mathcal{N}_{V, W}$ under $P \otimes_{\mathcal{R}} Q$ lies in $\mathcal{N}_{\widetilde{V}, \widetilde{W}}$, i.e. $P \otimes_{\mathcal{R}} Q\left(\mathcal{N}_{V, W}\right) \subseteq \mathcal{N}_{\widetilde{V}, \widetilde{W}}$. This is indeed the case because, for all $v \in V, w \in W$ and $a \in A$,

$$
\begin{aligned}
P \otimes_{\mathcal{R}} Q(v \cdot a \otimes w-v \otimes a & a w) \\
= & P\left(\bar{R}_{1}^{\alpha} \triangleright v \cdot \bar{R}_{2}^{\alpha} \triangleright a\right) \otimes\left(\bar{R}_{\alpha} \triangleright Q\right)(w) \\
& \quad P\left(\bar{R}^{\alpha} \triangleright v\right) \otimes\left(\bar{R}_{\alpha} \triangleright Q\right)(a \cdot w) \\
= & P\left(\bar{R}^{\alpha} \triangleright v\right) \cdot \bar{R}^{\beta} \triangleright a \otimes\left(\bar{R}_{\beta} \bar{R}_{\alpha} \triangleright Q\right)(w) \\
& \quad-P\left(\bar{R}^{\alpha} \triangleright v\right) \otimes \bar{R}^{\beta} \triangleright a \cdot\left(\bar{R}_{\beta} \bar{R}_{\alpha} \triangleright Q\right)(w) \in \mathcal{N}_{\widetilde{V}, \widetilde{W}},
\end{aligned}
$$

where in the last equality we used Proposition 5.14. The right $A$-linearity of the map $P \otimes_{\mathcal{R}} Q: V \otimes_{A} W \rightarrow \widetilde{V} \otimes_{A} \widetilde{W}$ follows from Proposition 5.5 and the fact that $V \otimes_{A} W$ (and $\widetilde{V} \otimes_{A} \widetilde{W}$ ) are equipped with the right $A$-module structure canonically induced from $V \otimes W$ (and $\widetilde{V} \otimes \widetilde{W}$ respectively). 
Theorem 5.16 immediately implies that the following definition is well-given.

Definition 5.17. Let $(H, \mathcal{R})$ be a quasitriangular Hopf algebra, $A$ be a quasi-commutative ${ }^{H} \mathscr{A}$-algebra, $V, \widetilde{V}$ be two ${ }^{H} \mathscr{M}_{A}$-modules and $W, \widetilde{W}$ be two quasi-commutative ${ }^{H}{ }_{A} \mathscr{M}_{A}$-modules. The $\mathcal{R}$-tensor product of right $A$-linear maps is defined by the $\mathbb{K}$-bilinear map

$$
\begin{aligned}
\otimes_{\mathcal{R}}: \operatorname{Hom}_{A}(V, \widetilde{V}) \times \operatorname{Hom}_{A}(W, \widetilde{W}) & \longrightarrow \operatorname{Hom}_{A}\left(V \otimes_{A} W, \widetilde{V} \otimes_{A} \widetilde{W}\right), \\
(P, Q) & \longmapsto P \otimes_{\mathcal{R}} Q,
\end{aligned}
$$

where $P \otimes_{\mathcal{R}} Q$ is explicitly given in (5.47).

Theorem 5.18. Let $(H, \mathcal{R})$ be a quasitriangular Hopf algebra, $A$ be a quasicommutative ${ }^{H} \mathscr{A}$-algebra, $V, \widetilde{V}, \widehat{V}$ be ${ }^{H} \mathscr{M}_{A}$-modules and $W, Z, \widetilde{W}, \widetilde{Z}, \widehat{W}$ be quasi-commutative ${ }_{A}{ }_{A} \mathscr{M}_{A}$-modules. The $\mathcal{R}$-tensor product of Definition 5.17 is compatible with the ${ }^{H} \mathscr{M}$-module structure, for all $\xi \in H, P \in \operatorname{Hom}_{A}(V$, $\widetilde{V})$ and $Q \in \operatorname{Hom}_{A}(W, \widetilde{W})$,

$$
\xi \triangleright\left(P \otimes_{\mathcal{R}} Q\right)=\left(\xi_{1} \triangleright P\right) \otimes_{\mathcal{R}}\left(\xi_{2} \triangleright Q\right) .
$$

Furthermore, the $\mathcal{R}$-tensor product is associative, for all $P \in \operatorname{Hom}_{A}(V, \widetilde{V})$, $Q \in \operatorname{Hom}_{A}(W, \widetilde{W})$ and $T \in \operatorname{Hom}_{A}(Z, \widetilde{Z})$,

$$
\left(P \otimes_{\mathcal{R}} Q\right) \otimes_{\mathcal{R}} T=P \otimes_{\mathcal{R}}\left(Q \otimes_{\mathcal{R}} T\right),
$$

and satisfies the braided composition law, for all $P \in \operatorname{Hom}_{A}(V, \widetilde{V}), Q \in$ $\operatorname{Hom}_{A}(W, \widetilde{W}), \widetilde{P} \in \operatorname{Hom}_{A}(\widetilde{V}, \widehat{V})$ and $\widetilde{Q} \in \operatorname{Hom}_{A}(\widetilde{W}, \widehat{W})$,

$$
\left(\widetilde{P} \otimes_{\mathcal{R}} \widetilde{Q}\right) \circ\left(P \otimes_{\mathcal{R}} Q\right)=\left(\widetilde{P} \circ\left(\bar{R}^{\alpha} \triangleright P\right)\right) \otimes_{\mathcal{R}}\left(\left(\bar{R}_{\alpha} \triangleright \widetilde{Q}\right) \circ Q\right) .
$$

Proof. These properties follow immediately from Theorem 5.4 because all maps in the present theorem are canonically induced from the maps in Theorem 5.4. Alternatively, one can repeat the calculations in the proof of Theorem 5.4 using the explicit expression (5.47) and acting on generating elements $v \otimes_{A} w \in V \otimes_{A} W$ or $v \otimes_{A} w \otimes_{A} z \in V \otimes_{A} W \otimes_{A} Z$.

\subsection{Deformation}

We study the twist deformation of the tensor product over $A$ of ${ }^{H} \mathscr{M}_{A^{-}}$ modules with ${ }^{H}{ }_{A} \mathscr{M}_{A}$-modules, and of right $A$-linear maps $P \otimes_{\mathcal{R}} Q$. Given ${ }^{H} \mathscr{M}_{A}$-modules $V, \widetilde{V}$ and ${ }^{H}{ }_{A} \mathscr{M}_{A}$-modules $W, \widetilde{W}$, Theorem 3.5 implies that 
we have the twist deformed ${ }^{H^{\mathcal{F}}} \mathscr{M}_{A_{\star}}$-modules $V_{\star}, \widetilde{V}_{\star}$ and the twist deformed $H_{A_{\star}} \mathscr{M}_{A_{\star}}$-modules $W_{\star}, \widetilde{W}_{\star}$, with $A_{\star}$ being the twist deformed ${ }^{H^{\mathcal{F}}} \mathscr{A}$-algebra. We can therefore consider $V_{\star} \otimes_{A_{\star}} W_{\star}$, that is the quotient of the $\mathbb{K}$-module $V_{\star} \otimes_{\star} W_{\star}$ via the $\mathbb{K}$-submodule $\mathcal{N}_{V_{\star}, W_{\star}}^{\star}$ generated by the elements $v \star a \otimes_{\star}$ $w-v \otimes_{\star} a \star w$, for all $a \in A_{\star}, v \in V_{\star}, w \in W_{\star}$. The image of $v \otimes_{\star} w$ under the canonical projection $\pi_{\star}: V_{\star} \otimes_{\star} W_{\star} \rightarrow V_{\star} \otimes_{A_{\star}} W_{\star}$ is denoted by $v \otimes_{A_{\star}} w$. Since the $\mathbb{K}$-submodule $\mathcal{N}_{V_{\star}^{\star}, W_{\star}}^{\star}$ is also an ${ }^{H^{\mathcal{F}}} \mathscr{M}_{A_{\star}}$-submodule, an ${ }^{H^{\mathcal{F}}} \mathscr{M}_{A_{\star}}$ module structure on $V_{\star} \otimes_{A_{\star}} W_{\star}^{\star}$ is canonically induced from the one on $V_{\star} \otimes_{\star}$ $W_{\star}$. Explicitly we have, for all $\xi \in H^{\mathcal{F}}, a \in A_{\star}, v \in V_{\star}, w \in W_{\star},\left(v \otimes_{A_{\star}} w\right) \star$ $a=v \otimes_{A_{\star}}(w \star a)$ and $\xi \triangleright_{\mathcal{F}}\left(v \otimes_{A_{\star}} w\right)=\left(\xi_{1_{\mathcal{F}}} \triangleright v\right) \otimes_{A_{\star}}\left(\xi_{2_{\mathcal{F}}} \triangleright w\right)$.

Lemma 5.19. Let $A$ be an ${ }^{H} \mathscr{A}$-algebra, $V$ be an ${ }^{H} \mathscr{M}_{A}$-module and $W$ be an ${ }^{H}{ }_{A} \mathscr{M}_{A}$-module. The $\mathbb{K}$-linear $\operatorname{map} \varphi_{V, W}=\mathcal{F}^{-1} \triangleright: V_{\star} \otimes_{\star} W_{\star} \rightarrow(V \otimes W)_{\star}$ defined in (5.27) is in this case an ${ }^{H^{\mathcal{F}}} \mathscr{M}_{A_{\star}}$-module isomorphism and induces a map on the quotients according to the following commutative diagram:

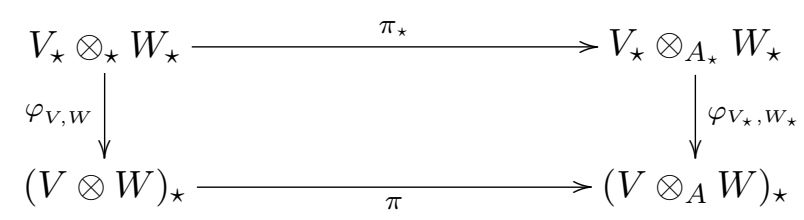

Explicitly,

$$
\begin{aligned}
& \varphi_{V_{\star}, W_{\star}}=\mathcal{F}^{-1} \triangleright: V_{\star} \otimes_{A_{\star}} W_{\star} \rightarrow\left(V \otimes_{A} W\right)_{\star}, \\
& v \otimes_{A_{\star}} w \mapsto \varphi_{V_{\star}, W_{\star}}\left(v \otimes_{A_{\star}} w\right)=\left(\overline{\mathrm{f}}^{\alpha} \triangleright v\right) \otimes_{A}\left(\overline{\mathrm{f}}_{\alpha} \triangleright w\right) .
\end{aligned}
$$

This map is an isomorphism between the ${ }^{H^{\mathcal{F}}} \mathscr{M}_{A_{\star}}$-modules $V_{\star} \otimes_{A_{\star}} W_{\star}$ and $\left(V \otimes_{A} W\right)_{\star}$.

Proof. The map $\varphi_{V, W}: V_{\star} \otimes_{\star} W_{\star} \rightarrow(V \otimes W)_{\star}$ was already shown to be an $H^{\mathcal{F}} \mathscr{M}$-module homomorphism in (5.28). It is an $H^{\mathcal{F}} \mathscr{M}_{A_{\star}}$-module homomorphism because, for all $v \in V_{\star}, w \in W_{\star}$ and $a \in A_{\star}$,

$$
\begin{aligned}
\varphi_{V, W}\left(v \otimes_{\star} w \star a\right) & =\overline{\mathrm{f}}^{\alpha} \triangleright v \otimes\left(\overline{\mathrm{f}}_{\alpha_{1}} \overline{\mathrm{f}}^{\beta} \triangleright w\right) \cdot\left(\overline{\mathrm{f}}_{\alpha_{2}} \overline{\mathrm{f}}_{\beta} \triangleright a\right) \\
& =\overline{\mathrm{f}}_{1}^{\alpha}{ }_{1} \overline{\mathrm{f}}^{\beta} \triangleright v \otimes\left(\overline{\mathrm{f}}_{\alpha_{2}} \overline{\mathrm{f}}_{\beta} \triangleright w\right) \cdot\left(\overline{\mathrm{f}}_{\alpha} \triangleright a\right) \\
& =\varphi_{V, W}\left(v \otimes_{\star} w\right) \star a,
\end{aligned}
$$

where we used the twist cocycle property $(3.4 \mathrm{c})$. It canonically induces the $\operatorname{map} \varphi_{V_{\star}, W_{\star}}$ in $(5.51)$ because it maps $\mathcal{N}_{V_{\star}, W_{\star}}^{\star}$ into $\mathcal{N}_{V, W}$, i.e. $\varphi_{V, W}\left(\mathcal{N}_{V_{\star}, W_{\star}}^{\star}\right) \subseteq$ 
$\mathcal{N}_{V, W}$. Indeed, for all $v \in V, w \in W$ and $a \in A$,

$$
\begin{aligned}
\varphi_{V, W} & \left(v \star a \otimes_{\star} w-v \otimes_{\star} a \star w\right) \\
= & \left(\overline{\mathrm{f}}_{1}^{\alpha} \overline{\mathrm{f}}^{\beta} \triangleright v\right) \cdot\left(\overline{\mathrm{f}}_{2}^{\alpha} \overline{\mathrm{f}}_{\beta} \triangleright a\right) \otimes \overline{\mathrm{f}}_{\alpha} \triangleright w \\
& \quad-\overline{\mathrm{f}}^{\alpha} \triangleright v \otimes\left(\overline{\mathrm{f}}_{\alpha_{1}} \overline{\mathrm{f}}^{\beta} \triangleright a\right) \cdot\left(\overline{\mathrm{f}}_{\alpha_{2}} \overline{\mathrm{f}}_{\beta} \triangleright w\right) \in \mathcal{N}_{V, W},
\end{aligned}
$$

because of the twist cocycle property (3.4c). The induced map $\varphi_{V_{\star}, W_{\star}}$ is an $H^{\mathcal{F}} \mathscr{M}_{A_{\star}}$-module homomorphism because so is $\varphi_{V, W}$, and $\mathcal{N}_{V_{\star}, W_{\star}}^{\star},\left(\mathcal{N}_{V, W}\right)_{\star}$ are ${ }^{H^{\mathcal{F}}} \mathscr{M}_{A_{\star}}$-submodules. (The ${ }^{H^{\mathcal{F}}} \mathscr{M}_{A_{\star}}$-module $\left(\mathcal{N}_{V, W}\right)_{\star}$ is obtained by applying Theorem 3.5 to the ${ }^{H} \mathscr{M}_{A}$-module $\mathcal{N}_{V, W}$. The corresponding projection $D_{\mathcal{F}}(\pi)=\pi$ is $H^{\mathcal{F}}$-equivariant and right $A_{\star}$-linear.) $\mathcal{F} \triangleright$.

Finally, the map (5.52) is obviously invertible with inverse $\varphi_{V_{\star}, W_{\star}}^{-1}:=$

Remark 5.20. If in the above lemma $V$ is an ${ }^{H_{A}} \mathscr{M}_{A}$-module, then the map $\varphi_{V, W}: V_{\star} \otimes_{\star} W_{\star} \rightarrow(V \otimes W)_{\star}$ is an ${ }^{H^{\mathcal{F}}}{ }_{A_{\star}} \mathscr{M}_{A_{\star}}$-module isomorphism. Furthermore $V_{\star} \otimes_{A_{\star}} W_{\star}$ is an ${ }^{H^{\mathcal{F}}}{ }_{A_{\star}} \mathscr{M}_{A_{\star}}$-module with left $A_{\star}$-module structure explicitly given by, for all $a \in A_{\star}, v \in V_{\star}, w \in W_{\star}, a \star\left(v \otimes_{A_{\star}} w\right)=(a \star$ $v) \otimes_{A_{\star}} w$. In this case the induced map $\varphi_{V_{\star}, W_{\star}}: V_{\star} \otimes_{A_{\star}} W_{\star} \rightarrow\left(V \otimes_{A} W\right)_{\star}$ is also an ${ }_{A_{\star}}^{H^{\mathcal{F}}} \mathscr{M}_{A_{\star}}$-module isomorphism.

Let now $A, W$ and $\widetilde{W}$ be also quasi-commutative. Then the commutative diagram (5.32) induces a commutative diagram on the corresponding quotient modules.

Theorem 5.21. Let $(H, \mathcal{R})$ be a quasitriangular Hopf algebra with twist $\mathcal{F} \in H \otimes H, A$ be a quasi-commutative ${ }^{H} \mathscr{A}$-algebra, $V, \widetilde{V}$ be two ${ }^{H} \mathscr{M}_{A^{-}}$ modules and $W, \widetilde{W}$ be two quasi-commutative ${ }_{A} \mathscr{M}_{A}$-modules. Then for all $P \in \operatorname{Hom}_{A}(V, \widetilde{V})$ and $Q \in \operatorname{Hom}_{A}(W, \widetilde{W})$ the following diagram of right $A_{\star}$-module homomorphisms commutes:

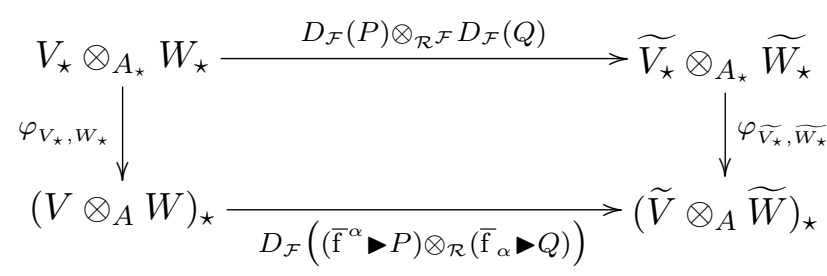

Proof. Theorems 5.16 and 4.7 imply that the horizontal arrows in the diagram are well-defined right $A_{\star}$-module homomorphisms. Lemma 5.19 states 
that the vertical arrows are ${ }^{H^{\mathcal{F}}} \mathscr{M}_{A_{\star}}$-module isomorphisms. The commutativity of the diagram (5.55) follows from the commutativity of the diagram (5.32) because the maps in (5.55) are all canonically induced by the maps in (5.32), (cf. (5.51)).

Remark 5.22. Theorem 5.21 can be interpreted as providing an equivalence of categories that have a tensor product over $A$ structure. Indeed, let us consider the category $\left({ }^{H} \mathscr{M}_{A}{ }^{\mathrm{qc}}, H_{0} \mathrm{~m}_{A}, \mathrm{o}\right)$, where objects are quasicommutative ${ }_{A} \mathscr{M}_{A}$-modules, morphisms are right $A$-linear maps and their composition is the usual composition. We equip this category with an "almost monoidal" structure $\otimes_{\mathcal{R}}$ given on objects $(V, W)$ by the tensor product over $A, V \otimes_{A} W$, and on morphisms $(P, Q)$ by $P \otimes_{\mathcal{R}} Q$. Lemma 5.19 implies that the isomorphisms $\varphi_{V_{\star}, W_{\star}}: V_{\star} \otimes_{A_{\star}} W_{\star} \rightarrow\left(V \otimes_{A} W\right)_{\star}$ satisfy a commutative diagram as in (5.30). Then Theorem 5.21 implies that the equivalence of categories found in Theorem 4.10 extends, as in Remark 5.7, to an equivalence of the corresponding "almost monoidal" categories $\left({ }^{H} \mathscr{M}_{A}{ }^{\mathrm{qc}}, \operatorname{Hom}_{A},{\mathrm{o}_{\star}}, \otimes_{\mathcal{R}_{\star}}\right)$ and $\left({ }^{H^{\mathcal{F}}}{ }_{A_{\star}} \mathscr{M}_{A_{\star}}{ }^{\mathrm{qc}}{ }_{\star}, \operatorname{Hom}_{A_{\star}}, \circ, \otimes_{\mathcal{R}^{\mathcal{F}}}\right)$.

\subsection{From right to left $A$-linear homomorphisms}

Left $A$-linear homomorphisms and endomorphisms of commutative $A$-bimodules over a commutative algebra $A$ are automatically also right $A$-linear and vice versa. If the Hopf algebra $(H, \mathcal{R})$ is quasitriangular, $A$ is a quasicommutative ${ }^{H} \mathscr{A}$-algebra and the ${ }^{H}{ }_{A} \mathscr{M}_{A}$-modules are strong quasi-commutative, we similarly have an isomorphism between right and left $A$-linear homomorphisms and endomorphisms. This can be shown by deforming ${ }^{H} \mathscr{A}-$ algebras and ${ }^{H} \mathscr{M}_{A}$-modules with the twist $\mathcal{F}=\mathcal{R}$.

Lemma 5.23. Let $(H, \mathcal{R})$ be a quasitriangular Hopf algebra with twist $\mathcal{F}=\mathcal{R}, A$ be a quasi-commutative ${ }^{H} \mathscr{A}$-algebra and $V$ be a strong quasicommutative ${ }^{H}{ }_{A} \mathscr{M}_{A}$-module. Then $H^{\mathcal{R}}=H^{\text {cop }}, A_{\star_{\mathcal{R}}}=A^{o p}$ and $V_{\star_{\mathcal{R}}}=V^{o p}$. Here $\star_{\mathcal{R}}$ denotes the deformation associated with the twist $\mathcal{F}=\mathcal{R}$.

Proof. The bialgebras $H^{\mathcal{R}}$ and $H^{\text {cop }}$ are the same because the $\mathcal{R}$-twisted coproduct is the coopposite coproduct (cf. (5.2)). Uniqueness of the antipode implies that they are the same Hopf algebra.

$A_{\star_{\mathcal{R}}}=A^{o p}$ as algebras (and hence as $H^{\text {cop }} \mathscr{A}$-algebras) because, for all $a, \tilde{a} \in A, a \star_{\mathcal{R}} \tilde{a}=\left(\bar{R}^{\alpha} \triangleright a\right)\left(\bar{R}_{\alpha} \triangleright \tilde{a}\right)=\tilde{a} a=\mu^{o p}(a \otimes \tilde{a})$. 
Similarly $V_{\star_{\mathcal{R}}}=V^{o p}$ as $A^{o p}$-bimodules (and hence as ${ }^{H^{\text {cop }}}{ }_{A^{o p}} \mathscr{M}_{A^{\text {op }} \text {-mod- }}$ ules) because, for all $a \in A, v \in V$,

$$
\begin{aligned}
& a \star_{\mathcal{R}} v=\bar{R}^{\alpha}(a) \cdot \bar{R}_{\alpha}(v)=v \cdot a=a \cdot{ }^{o p} v, \\
& v \star_{\mathcal{R}} a=\bar{R}^{\alpha}(v) \cdot \bar{R}_{\alpha}(a)=a \cdot v=v \cdot{ }^{o p} a,
\end{aligned}
$$

where in the first equality we have used the quasi-commutativity condition (5.41) and in the second equality the inverse of the strong quasicommutativity condition (5.43).

Remark 5.24. Lemma 5.23 holds true also if we use the alternative $\mathcal{R}$ matrix $\mathcal{R}_{21}^{-1}$ as a twist, i.e. if we use $\mathcal{F}=\mathcal{R}_{21}^{-1}$.

Theorem 5.25. Let $(H, \mathcal{R})$ be a quasitriangular Hopf algebra with twist $\mathcal{F}=\mathcal{R}, A$ be a quasi-commutative ${ }^{H} \mathscr{A}$-algebra and $V, W$ be two strong quasicommutative ${ }_{A} \mathscr{M}_{A}$-modules. Then there is an isomorphism (that with slight abuse of notation we denote)

$$
\begin{aligned}
D_{\mathcal{R}}:\left(\operatorname{End}_{A}(V)_{\star_{\mathcal{R}}}\right)^{o p} & \longrightarrow\left({ }_{A} \operatorname{End}(V)\right)^{o p} \\
P & \longmapsto D_{\mathcal{R}}(P):=\left(\bar{R}^{\alpha} \triangleright P\right) \circ \bar{R}_{\alpha} \triangleright
\end{aligned}
$$

between the ${ }^{H} \mathscr{A}_{A}$-algebras $\left(\operatorname{End}_{A}(V), \circ_{\star_{\mathcal{R}}}{ }^{o p}, \star_{\mathcal{R}}{ }^{o p}, \boldsymbol{\bullet}\right)$ and $\left({ }_{A} \operatorname{End}(V), \circ^{o p}\right.$, $\left.{ }^{o p},{ }^{c o p}\right)$.

Similarly there is an isomorphism (denoted by the same symbol)

$$
\begin{aligned}
D_{\mathcal{R}}: \operatorname{Hom}_{A}(V, W) & \longrightarrow\left({ }_{A} \operatorname{Hom}(V, W)\right)^{o p} \\
P & \longmapsto D_{\mathcal{R}}(P):=\left(\bar{R}^{\alpha} \triangleright P\right) \circ \bar{R}_{\alpha} \triangleright
\end{aligned}
$$

between the ${ }_{A} \mathscr{M}_{A}$-modules $\left(\operatorname{Hom}_{A}(V, W), \cdot, \nabla\right)$ and $\left({ }_{A} \operatorname{Hom}(V, W),{ }^{o p},{ }^{c o p}\right)$.

Proof. From $H^{\mathcal{R}}=H^{\text {cop }}$ we have the equality of the corresponding adjoint actions $\triangleright_{\mathcal{R}} \Rightarrow{ }^{c o p}$. From $A_{\star_{\mathcal{R}}}=A^{o p}$ and $V_{\star_{\mathcal{R}}}=V^{o p}$ we have $\operatorname{End}_{A_{\star_{\mathcal{R}}}}\left(V_{\star_{\mathcal{R}}}\right)=$ $\operatorname{End}_{A^{o p}}\left(V^{o p}\right)$, i.e., more explicitly, $\left(\operatorname{End}_{A_{\star_{\mathcal{R}}}}\left(V_{\star_{\mathcal{R}}}\right), \circ, \cdot, \boldsymbol{R}_{\mathcal{R}}\right)=\left(\operatorname{End}_{A^{o p}}\left(V^{o p}\right)\right.$,

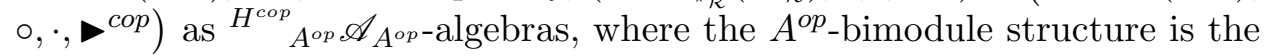
usual one obtained with the left multiplication map, that in this case is $l^{A^{o p}}: A^{o p} \rightarrow \operatorname{End}_{A^{o p}}\left(V^{o p}\right)$. Recalling the canonical isomorphism $\operatorname{End}_{A^{o p}}\left(V^{o p}\right)$ $\simeq{ }_{A} \operatorname{End}(V) \quad(\mathrm{cf} . \quad(4.18)) \quad$ we have $\left(\operatorname{End}_{A_{\star_{\mathcal{R}}}}\left(V_{\star_{\mathcal{R}}}\right), \circ, \cdot, \boldsymbol{}_{\mathcal{R}}\right) \simeq\left({ }_{A} \operatorname{End}(V)\right.$, $\left.\circ, \cdot \triangleright^{c o p}\right)$. Use of Lemma 4.3 leads to the ${ }_{A}{ }_{A} \mathscr{A}_{A}$-algebra isomorphism $\left(\operatorname{End}_{A_{\star_{\mathcal{R}}}}\left(V_{\star_{\mathcal{R}}}\right)\right)^{o p} \simeq\left({ }_{A} \operatorname{End}(V)\right)^{o p}$. The isomorphism $D_{\mathcal{R}}: \operatorname{End}_{A}(V)_{\star_{\mathcal{R}}} \rightarrow$ $\operatorname{End}_{A_{\star_{\mathcal{R}}}}\left(V_{\star_{\mathcal{R}}}\right)$ of Theorem 4.2 between the ${ }^{H^{\mathcal{R}}}{ }_{A_{\star_{\mathcal{R}}}} \mathscr{A}_{A_{\star_{\mathcal{R}}}}$-algebras $\left(\operatorname{End}_{A}(V)\right.$, 
$\left.\circ_{\star_{\mathcal{R}}}, \star_{\mathcal{R}}, \triangleright\right)$ and $\left(\operatorname{End}_{A_{\star_{\mathcal{R}}}}\left(V_{\star_{\mathcal{R}}}\right), \circ, \cdot, \boldsymbol{}_{\mathcal{R}}\right)$ then induces the ${ }^{H}{ }_{A} \mathscr{A}_{A}$-algebra isomorphism

$$
D_{\mathcal{R}}:\left(\operatorname{End}_{A}(V)_{\star_{\mathcal{R}}}\right)^{o p} \longrightarrow\left({ }_{A} \operatorname{End}(V)\right)^{o p}
$$

between $\left(\operatorname{End}_{A}(V), \circ_{\star_{\mathcal{R}}}{ }^{o p}, \star_{\mathcal{R}}^{o p}, \triangleright\right)$ and $\left({ }_{A} \operatorname{End}(V), \circ^{o p},{ }^{o p},{ }^{c o p}\right)$.

The construction of the isomorphism $D_{\mathcal{R}}$ for homomorphisms is similar and leads to an ${ }^{H} \mathscr{M}_{A}$-module isomorphism

$$
D_{\mathcal{R}}:\left(\operatorname{Hom}_{A}(V, W)_{\star_{\mathcal{R}}}\right)^{o p} \longrightarrow\left({ }_{A} \operatorname{Hom}(V, W)\right)^{o p} .
$$

We conclude the proof by showing that as ${ }^{H}{ }_{A} \mathscr{M}_{A}$-modules

$$
\left(\operatorname{Hom}_{A}(V, W)_{\star_{\mathcal{R}}}\right)^{o p}=\operatorname{Hom}_{A}(V, W) .
$$

We apply Lemma 5.23 to the strong quasi-commutative ${ }_{A} \mathscr{M}_{A}$-module $\operatorname{Hom}_{A}(V, W)$, and obtain $\operatorname{Hom}_{A}(V, W)_{\star_{\mathcal{R}}}=\operatorname{Hom}_{A}(V, W)^{o p}$. Therefore, $\left(\operatorname{Hom}_{A}(V, W)_{\star_{\mathcal{R}}}\right)^{o p}=\operatorname{Hom}_{A}(V, W)^{o p o p}=\operatorname{Hom}_{A}(V, W)$.

In Theorems 4.7 and 4.8 we defined the deformation maps $D_{\mathcal{F}}$ and $D_{\mathcal{F}}^{c o p}$ between homomorphisms of ${ }^{H^{\mathcal{F}}}{ }_{A_{\star}} \mathscr{M}_{A_{\star}}$-modules (see also the corresponding theorems for endomorphisms). In Theorem 5.25 with slight abuse of notation we have still denoted by $D_{\mathcal{R}}$ the deformation map that is now between homomorphisms of ${ }^{H} \mathscr{M}_{A}$-modules (rather than ${ }^{H^{\mathcal{R}}}{ }_{A_{\star_{\mathcal{R}}}} \mathscr{M}_{A_{\star_{\mathcal{R}}}}$-modules).

The right to left isomorphism is compatible with twist deformation.

Theorem 5.26. Let $(H, \mathcal{R})$ be a quasitriangular Hopf algebra with twist $\mathcal{F} \in H \otimes H, A$ a quasi-commutative ${ }^{H} \mathscr{A}$-algebra and $V, W$ two strong quasicommutative ${ }_{A}{ }_{A} \mathscr{M}_{A}$-modules. Then the following diagram of ${ }^{H^{\mathcal{F}}}{ }_{A_{\star}} \mathscr{M}_{A_{\star}}$-module isomorphisms commutes:

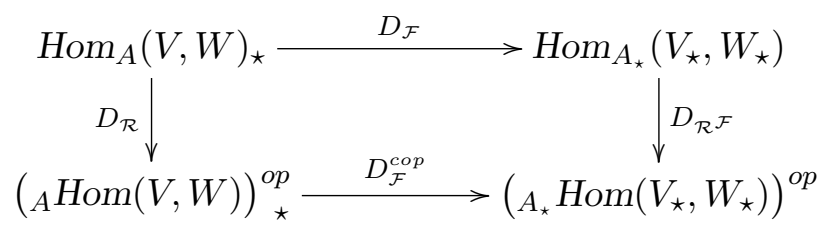

Proof. $H$-equivariance of $D_{\mathcal{R}}$ in (5.58) implies that $D_{\mathcal{R}}: \operatorname{Hom}_{A}(V, W)_{\star} \rightarrow$ $\left({ }_{A} \operatorname{Hom}(V, W)\right)_{\star}^{o p}$ is an ${ }^{H^{\mathcal{F}}} A_{\star} \mathscr{M}_{A_{\star}}$-module isomorphism $\left(D_{\mathcal{R}}=D_{\mathcal{F}}\left(D_{\mathcal{R}}\right)=\right.$ $\left.D_{\mathcal{F}}^{\text {cop }}\left(D_{\mathcal{R}}\right)\right)$.

Since all maps in the diagram are ${ }^{H^{\mathcal{F}}}{ }_{A_{\star}} \mathscr{M}_{A_{\star}}$-module isomorphisms, its commutativity is proven if we prove the equality $D_{\mathcal{R}^{\mathcal{F}}}=D_{\mathcal{F}}^{\text {cop }} \circ D_{\mathcal{R}} \circ D_{\mathcal{F}}^{-1}$ 
as $\mathbb{K}$-linear maps. This immediately follows from $\mathcal{R}^{\mathcal{F}}=\mathcal{F}_{21} \mathcal{R} \mathcal{F}^{-1}$ and the equality $D_{\mathcal{F}^{\prime} \mathcal{F}}=D_{\mathcal{F}^{\prime}} \circ D_{\mathcal{F}}$, where $\mathcal{F}$ is a twist of $H, \mathcal{F}^{\prime}$ is a twist of $H^{\mathcal{F}}$, and hence, as it is easily seen, $\mathcal{F}^{\prime} \mathcal{F}$ is a twist of $H$. We obtain, for all $P \in \operatorname{Hom}_{A}(V, W)$,

$$
\begin{aligned}
D_{\mathcal{F}^{\prime} \mathcal{F}}(P) & =\left(\overline{\mathrm{f}^{\alpha}}{\overline{\mathrm{f}^{\prime}}}^{\beta}-P\right) \circ \overline{\mathrm{f}_{\alpha}} \overline{\mathrm{f}^{\prime}}{ }_{\beta}^{\triangleright}=D_{\mathcal{F}}\left({\overline{\mathrm{f}^{\prime}}}^{\beta}-P\right) \circ \overline{\mathrm{f}^{\prime}}{ }_{\beta}^{\triangleright} \\
& =\left(\overline{\mathrm{f}^{\prime}}{ }^{\beta} D_{\mathcal{F}} D_{\mathcal{F}}(P)\right) \circ \overline{\mathrm{f}^{\prime}}{ }_{\beta} \triangleright=D_{\mathcal{F}^{\prime}}\left(D_{\mathcal{F}}(P)\right),
\end{aligned}
$$

where we used that $D_{\mathcal{F}}$ intertwines between the $\boldsymbol{\sim}$ and $\boldsymbol{\mathcal { F }}_{\mathcal{F}}$ action.

Example 5.27. Let $(H, \mathcal{R})$ be a quasitriangular Hopf algebra, $A$ be a quasicommutative ${ }^{H} \mathscr{A}$-algebra and $V$ be a strong quasi-commutative ${ }^{H}{ }_{A} \mathscr{M}_{A^{-}}$ module. Then by Theorem 5.25 there is an ${ }^{H}{ }_{A} \mathscr{M}_{A}$-module isomorphism between the right dual $V^{\prime}=\operatorname{Hom}_{A}(V, A)$ and the left dual ${ }^{\prime} V=\left({ }_{A} \operatorname{Hom}(V\right.$, A) $)^{o p}$.

\section{Connections}

We review the notion of connections on $\mathscr{M}_{A}$-modules and $A \mathscr{M}$-modules. For ${ }^{H} \mathscr{A}$-algebras $A$ equipped with a suitable $H$-covariant differential calculus and ${ }^{H} \mathscr{M}_{A}$-modules (and also ${ }^{H}{ }_{A} \mathscr{M}$-modules or ${ }^{H}{ }_{A} \mathscr{M}_{A}$-modules) we prove that there is a bijective correspondence between connections on the undeformed and deformed modules. It is an isomorphism between the undeformed and deformed affine spaces of connections. We then investigate the problem of constructing connections on tensor products of ${ }^{H_{A}} \mathscr{M}_{A}$-modules from connections on the individual factors. Assuming, as in the study of tensor product module homomorphisms, quasi-commutativity of the ${ }^{H} \mathscr{A}$-algebras and ${ }_{A}{ }_{A} \mathscr{M}_{A}$-modules, we define a sum of arbitrary (i.e. not necessarily $H$ equivariant) connections which yields a connection on the tensor product module. The twist deformation of this sum is investigated in detail. As in the case of module homomorphisms we can use the twist $\mathcal{F}=\mathcal{R}$ in order to identify right with left connections on strong quasi-commutative ${ }_{A}{ }_{A} \mathscr{M}_{A^{-}}$ modules. Finally, the construction and twist deformation of connections on the dual module is studied and shown to be canonical. An extension of connections to the tensor algebra of a module and its dual is given.

\subsection{Connections on right and left modules}

We briefly review the notion of a connection on an $\mathscr{M}_{A}$-module or ${ }_{A} \mathscr{M}$ module, see e.g. [15, 23] for an introduction. 
Definition 6.1. Let $A$ be an algebra. A differential calculus $\left(\Omega^{\bullet}, \wedge, \mathrm{d}\right)$ over $A$ (or an $\mathbb{N}^{0}$-differential graded algebra) is an $\mathbb{N}^{0}$-graded algebra $\left(\Omega^{\bullet}=\bigoplus_{n \geq 0} \Omega^{n}, \wedge\right)$, where $\Omega^{0}=A$ has degree zero, together with a $\mathbb{K}$-linear map $\mathrm{d}: \Omega^{\bullet} \rightarrow \Omega^{\bullet}$ of degree one, satisfying $\mathrm{d} \circ \mathrm{d}=0$ and the graded Leibniz rule

$$
\mathrm{d}\left(\omega \wedge \omega^{\prime}\right)=(\mathrm{d} \omega) \wedge \omega^{\prime}+(-1)^{\operatorname{deg}(\omega)} \omega \wedge\left(\mathrm{d} \omega^{\prime}\right),
$$

for all $\omega, \omega^{\prime} \in \Omega^{\bullet}$ with $\omega$ of homogeneous degree.

The differential $\mathrm{d}$ and the product $\wedge$ give rise to $\mathbb{K}$-linear maps (denoted by the same symbols) $\mathrm{d}: \Omega^{n} \rightarrow \Omega^{n+1}$ and $\wedge: \Omega^{n} \otimes \Omega^{m} \rightarrow \Omega^{n+m}$. Note that in the hypotheses above the $\mathbb{K}$-modules $\Omega^{n}$ are ${ }_{A} \mathscr{M}_{A}$-modules. As in commutative differential geometry we call $\Omega^{n}$ the module of $n$-forms, notice however that our wedge product $\wedge$ is not necessarily graded commutative. We also assume that any 1-form $\theta \in \Omega:=\Omega^{1}$ can be written as $\theta=\sum_{i} a_{i} \mathrm{~d} b_{i}$, with $a_{i}, b_{i} \in A$, i.e. that exact 1 -forms generate $\Omega$ as an ${ }_{A} \mathscr{M}$-module.

Example 6.2. Let $M$ be a $D$-dimensional smooth (second countable) manifold and let $A=C^{\infty}(M)$ be the smooth and complex (or real) valued functions on $M$. The exterior algebra of differential forms $\left(\Omega^{\bullet}=\bigoplus_{n>0} \Omega^{n}, \wedge\right)$ is an $\mathbb{N}^{0}$-graded algebra over $\mathbb{C}$ (or $\mathbb{R}$ ), where $\Omega^{0}=A$ and $\Omega^{n}=\overline{0}$, for all $n>D$. The exterior differential d is a differential on $\left(\Omega^{\bullet}, \wedge\right)$, leading to the de Rham differential calculus $\left(\Omega^{\bullet}, \wedge, \mathrm{d}\right)$. In this special case $\Omega^{\bullet}$ is graded commutative.

Another example is given by the twist deformed differential calculus $\left(\Omega^{\bullet}[[h]], \wedge_{\star}, \mathrm{d}\right)[6]$. There, the algebra $\left(\Omega^{\bullet}[[h]], \wedge_{\star}\right)$ over $\mathbb{K}=\mathbb{C}[[h]]$ is graded quasi-commutative, i.e., for all $\omega, \omega^{\prime} \in \Omega^{\bullet}$ of homogeneous degree,

$$
\omega \wedge_{\star} \omega^{\prime}=(-1)^{\operatorname{deg}(\omega) \operatorname{deg}\left(\omega^{\prime}\right)}\left(\bar{R}^{\alpha} \triangleright \omega^{\prime}\right) \wedge_{\star}\left(\bar{R}_{\alpha} \triangleright \omega\right) .
$$

Definition 6.3. Let $A$ be an algebra and $\left(\Omega^{\bullet}, \wedge, \mathrm{d}\right)$ be a differential calculus over $A$. A connection on an $\mathscr{M}_{A}$-module $V$ is a $\mathbb{K}$-linear map $\nabla: V \rightarrow V \otimes_{A} \Omega$, satisfying the right Leibniz rule, for all $v \in V$ and $a \in A$,

$$
\nabla(v \cdot a)=(\nabla v) \cdot a+v \otimes_{A} \mathrm{~d} a .
$$

Similarly, a connection on an $A \mathscr{M}$-module $V$ is a $\mathbb{K}$-linear map $\nabla: V \rightarrow$ $\Omega \otimes_{A} V$, satisfying the left Leibniz rule, for all $v \in V$ and $a \in A$,

$$
\nabla(a \cdot v)=a \cdot(\nabla v)+\mathrm{d} a \otimes_{A} v .
$$


In case $V$ is an ${ }_{A} \mathscr{M}_{A}$-module we say that a $\mathbb{K}$-linear map $\nabla: V \rightarrow V \otimes_{A} \Omega$ is a right connection on $V$ if $(6.3)$ is satisfied. Similarly, we say that a $\mathbb{K}$ linear map $\nabla: V \rightarrow \Omega \otimes_{A} V$ is a left connection on $V$ if (6.4) is satisfied.

We denote by $\operatorname{Con}_{A}(V)$ the set of all connections on an $\mathscr{M}_{A}$-module $V$ and by ${ }_{A} \operatorname{Con}(V)$ the set of all connections on an ${ }_{A} \mathscr{M}$-module $V$. We also denote by $\operatorname{Con}_{A}(V)$ and ${ }_{A} \operatorname{Con}(V)$, respectively, the set of all right and left connections on an $\mathscr{M}_{A}$-module $V$. Note that given any connection $\nabla \in$ $\mathrm{Con}_{A}(V)$ and any right $A$-linear map $P \in \operatorname{Hom}_{A}\left(V, V \otimes_{A} \Omega\right)$, then $\widetilde{\nabla}=\nabla+$ $P \in \operatorname{Con}_{A}(V)$ is again a connection. Indeed, for all $a \in A$ and $v \in V$,

$$
\begin{aligned}
\widetilde{\nabla}(v \cdot a) & =\nabla(v \cdot a)+P(v \cdot a)=(\nabla v) \cdot a+P(v) \cdot a+v \otimes_{A} \mathrm{~d} a \\
& =(\widetilde{\nabla} v) \cdot a+v \otimes_{A} \mathrm{~d} a
\end{aligned}
$$

The action $\nabla \mapsto \nabla+P$ is free and transitive and hence $\operatorname{Con}_{A}(V)$ is an affine space over the $\mathbb{K}$-module $\operatorname{Hom}_{A}\left(V, V \otimes_{A} \Omega\right)$. Similarly, the space of left connections ${ }_{A} \operatorname{Con}(V)$ is an affine space over the $\mathbb{K}$-module ${ }_{A} \operatorname{Hom}(V$, $\left.\Omega \otimes_{A} V\right)$.

\subsection{Deformation of connections}

We now consider differential calculi that are also $H$-covariant and study the twist deformation of connections on ${ }^{H}{ }_{A} \mathscr{M}$-modules and ${ }^{H} \mathscr{M}_{A}$-modules.

Let $H$ be a Hopf algebra and $A$ be an ${ }^{H} \mathscr{A}$-algebra. Let further $\left(\Omega^{\bullet}, \wedge, \mathrm{d}\right)$ be a left $H$-covariant differential calculus over $A$, i.e., $\Omega^{\bullet}$ is an ${ }^{H} \mathscr{A}$ algebra, the $H$-action $\triangleright$ is degree preserving and the differential is $H$-equivariant, for all $\xi \in H$ and $\omega \in \Omega^{\bullet}$,

$$
\xi \triangleright(\mathrm{d} \omega)=\mathrm{d}(\xi \triangleright \omega)
$$

Since the $H$-action is degree preserving, $\Omega^{n}$ are ${ }^{H}{ }_{A} \mathscr{M}_{A}$-modules, for all $n$ $\in \mathbb{N}^{0}$.

We now show that a left $H$-covariant differential calculus can be deformed to yield a left $H^{\mathcal{F}}$-covariant differential calculus.

Proposition 6.4. Let $H$ be a Hopf algebra with twist $\mathcal{F} \in H \otimes H, A$ be an $H_{\mathscr{A}}$-algebra and $\left(\Omega^{\bullet}, \wedge, \mathrm{d}\right)$ be a left $H$-covariant differential calculus over $A$. Then $\left(\Omega^{\bullet}, \wedge_{\star}, \mathrm{d}\right)$ is a left $H^{\mathcal{F}}$-covariant differential calculus over the $H^{\mathcal{F}} \mathscr{A}$ algebra $A_{\star}$. 
Proof. By Theorem $3.4\left(\Omega^{\bullet}, \wedge_{\star}\right)$ is an $H^{\mathcal{F}} \mathscr{A}$-algebra. It is $\mathbb{N}^{0}$-graded and we have $\left(\Omega^{0}, \wedge_{\star}\right)=A_{\star}$. Due to the $H$-equivariance of the differential, $\mathrm{d}$ is also a differential on $\left(\Omega^{\bullet}, \wedge_{\star}\right)$.

Notice that due to $H$-equivariance of the differential d the de Rham complex is invariant under twist deformation.

Right modules. Let $V$ be an ${ }^{H} \mathscr{M}_{A}$-module. Since $\operatorname{Con}_{A}(V) \subseteq \operatorname{Hom}_{\mathbb{K}}(V$, $\left.V \otimes_{A} \Omega\right)$ we can act with the $H$-adjoint action $\triangleright$ on $\nabla \in \operatorname{Con}_{A}(V)$, for all $\xi \in H$,

$$
\xi \triangleright \nabla:=\xi_{1} \triangleright \circ \nabla \circ S\left(\xi_{2}\right) \triangleright
$$

The element $\xi \triangleright \nabla \in \operatorname{Hom}_{\mathbb{K}}\left(V, V \otimes_{A} \Omega\right)$ satisfies, for all $v \in V$ and $a \in A$,

$$
\begin{aligned}
(\xi \triangleright \nabla)(v \cdot a)= & \xi_{1} \triangleright\left(\nabla\left(S\left(\xi_{2}\right)_{1} \triangleright v \cdot S\left(\xi_{2}\right)_{2} \triangleright a\right)\right) \\
= & \xi_{1} \triangleright\left(\left(\nabla\left(S\left(\xi_{3}\right) \triangleright v\right)\right) \cdot\left(S\left(\xi_{2}\right) \triangleright a\right)\right. \\
& \left.\quad+\left(S\left(\xi_{3}\right) \triangleright v\right) \otimes_{A} \mathrm{~d}\left(S\left(\xi_{2}\right) \triangleright a\right)\right) \\
= & ((\xi \triangleright \nabla)(v)) \cdot a+\varepsilon(\xi) v \otimes_{A} \mathrm{~d} a .
\end{aligned}
$$

In particular we see that if $\varepsilon(\xi)=0$ then $\xi \nabla \nabla \in \operatorname{Hom}_{A}\left(V, V \otimes_{A} \Omega\right.$ ), while if $\varepsilon(\xi)=1$ then $\xi \nabla \nabla \in \operatorname{Con}_{A}(V)$.

We now show that given a twist $\mathcal{F} \in H \otimes H$ of the Hopf algebra $H$, then there is an isomorphism $\operatorname{Con}_{A}(V) \simeq \operatorname{Con}_{A_{\star}}\left(V_{\star}\right)$, between connections on the undeformed ${ }^{H} \mathscr{M}_{A}$-module $V$ and on the deformed ${ }^{H^{\mathcal{F}}} \mathscr{M}_{A_{\star}}$-module $V_{\star}$.

We first observe that by composing any $\mathbb{K}$-linear map $V_{\star} \rightarrow\left(V \otimes_{A} \Omega\right)_{\star}$ with the ${ }^{H^{\mathcal{F}}} \mathscr{M}_{A_{\star}}$-module isomorphism

$$
\varphi^{-1}:\left(V \otimes_{A} \Omega\right)_{\star} \longrightarrow V_{\star} \otimes_{A_{\star}} \Omega_{\star}
$$

studied in Lemma 5.19 (for a simpler notation we drop the module indices on $\varphi$ ) we obtain the ${ }^{H^{\mathcal{F}}} \mathscr{M}$-module isomorphism (that with abuse of notation we still denote by $\varphi^{-1}$ )

$$
\varphi^{-1}: \operatorname{Hom}_{\mathbb{K}}\left(V_{\star},\left(V \otimes_{A} \Omega\right)_{\star}\right) \longrightarrow \operatorname{Hom}_{\mathbb{K}}\left(V_{\star}, V_{\star} \otimes_{A_{\star}} \Omega_{\star}\right) .
$$

Composition of the map $D_{\mathcal{F}}: \operatorname{Hom}_{\mathbb{K}}\left(V, V \otimes_{A} \Omega\right)_{\star} \longrightarrow H^{\mathcal{F}} \mathrm{m}_{\mathbb{K}}\left(V_{\star},\left(V \otimes_{A} \Omega\right)_{\star}\right)$ with this isomorphism gives the ${ }^{H^{\mathcal{F}}} \mathscr{M}$-module isomorphism

$$
\widetilde{D}_{\mathcal{F}}:=\varphi^{-1} \circ D_{\mathcal{F}}: \operatorname{Hom}_{\mathbb{K}}\left(V, V \otimes_{A} \Omega\right)_{\star} \longrightarrow \operatorname{Hom}_{\mathbb{K}}\left(V_{\star}, V_{\star} \otimes_{A_{\star}} \Omega_{\star}\right)
$$


Next we define $\operatorname{Con}_{A}(V)_{\star}$ to be the same set of connections as $\operatorname{Con}_{A}(V)$, but with affine space structure over the ${ }^{H^{\mathcal{F}}} \mathscr{M}$-module $\operatorname{Hom}_{A}\left(V, V \otimes_{A} \Omega\right)_{\star}$ rather than over the ${ }^{H} \mathscr{M}$-module $\operatorname{Hom}_{A}\left(V, V \otimes_{A} \Omega\right.$ ) (we recall that they coincide as $\mathbb{K}$-modules).

Theorem 6.5. Let $H$ be a Hopf algebra with twist $\mathcal{F} \in H \otimes H, A$ be an ${ }^{H} \mathscr{A}$-algebra, $V$ be an ${ }^{H} \mathscr{M}_{A}$-module and $\left(\Omega^{\bullet}, \wedge, \mathrm{d}\right)$ be a left $H$-covariant differential calculus over $A$. The ${ }^{H^{\mathcal{F}}} \mathscr{M}$-module isomorphism (6.11) restricts to the ${ }^{H^{\mathcal{F}}} \mathscr{M}$-module isomorphism

$$
\begin{aligned}
\widetilde{D}_{\mathcal{F}}: \operatorname{Hom}_{A}\left(V, V \otimes_{A} \Omega\right)_{\star} & \longrightarrow \operatorname{Hom}_{A_{\star}}\left(V_{\star}, V_{\star} \otimes_{A_{\star}} \Omega_{\star}\right), \\
P & \longmapsto \varphi^{-1} \circ\left(\overline{\mathrm{f}}^{\alpha} \triangleright P\right) \circ \overline{\mathrm{f}}_{\alpha} \triangleright
\end{aligned}
$$

and to the affine space isomorphism

$$
\begin{aligned}
\widetilde{D}_{\mathcal{F}}: \operatorname{Con}_{A}(V)_{\star} & \longrightarrow \operatorname{Con}_{A_{\star}}\left(V_{\star}\right) \\
\nabla & \longmapsto \varphi^{-1} \circ\left(\overline{\mathrm{f}}^{\alpha} \triangleright \nabla\right) \circ \overline{\mathrm{f}}_{\alpha} \triangleright
\end{aligned}
$$

where $\operatorname{Con}_{A}(V)_{\star}$ and $\operatorname{Con}_{A_{\star}}\left(V_{\star}\right)$ are respectively affine spaces over the isomorphic ${ }^{H^{\mathcal{F}}} \mathscr{M}$-modules $\operatorname{Hom}_{A}\left(V, V \otimes_{A} \Omega\right)_{\star}$ and $\operatorname{Hom}_{A_{\star}}\left(V_{\star}, V_{\star} \otimes_{A_{\star}} \Omega_{\star}\right)$.

Proof. From Lemma 5.19 we know that the map (6.9) is an ${ }^{H^{\mathcal{F}}} \mathscr{M}_{A_{\star}}$-module isomorphism. It then follows that the ${ }^{H^{\mathcal{F}}} \mathscr{M}$-module isomorphism $\varphi^{-1}$ in (6.10) restricts to an $H^{\mathcal{F}} \mathscr{M}$-module isomorphism (still denoted by $\varphi^{-1}$ ),

$$
\varphi^{-1}: \operatorname{Hom}_{A_{\star}}\left(V_{\star},\left(V \otimes_{A} \Omega\right)_{\star}\right) \longrightarrow \operatorname{Hom}_{A_{\star}}\left(V_{\star}, V_{\star} \otimes_{A_{\star}} \Omega_{\star}\right)
$$

Henceforth, as in the case of the isomorphism $D_{\mathcal{F}}$ (cf. Theorem 4.2), the isomorphism $\widetilde{D}_{\mathcal{F}}$ in (6.11) restricts to the ${ }^{H^{\mathcal{F}}} \mathscr{M}$-module isomorphism (still denoted $\widetilde{D}_{\mathcal{F}}$ )

$$
\widetilde{D}_{\mathcal{F}}: \operatorname{Hom}_{A}\left(V, V \otimes_{A} \Omega\right)_{\star} \longrightarrow \operatorname{Hom}_{A_{\star}}\left(V_{\star}, V_{\star} \otimes_{A_{\star}} \Omega_{\star}\right) .
$$

The proof that $\widetilde{D}_{\mathcal{F}}$ in $(6.13)$ maps connections into connections is similar to the proof of the right $A_{\star}$-linearity of the map $D_{\mathcal{F}}(P)$, when $P$ is a right $A$-linear map (cf. Theorem 4.2): Let $\nabla \in \operatorname{Con}_{A}(V)_{\star}$, then, for all $v \in V$ and $a \in A$, 


$$
\begin{aligned}
D_{\mathcal{F}}(\nabla)(v \star a)= & \left(\overline{\mathrm{f}}^{\alpha} \triangleright \nabla\right)\left(\left(\overline{\mathrm{f}}_{\alpha_{1}} \overline{\mathrm{f}}^{\beta} \triangleright v\right) \cdot\left(\overline{\mathrm{f}}_{\alpha_{2}} \overline{\mathrm{f}}_{\beta} \triangleright a\right)\right) \\
= & \left(\left(\overline{\mathrm{f}}^{\alpha} \triangleright \nabla\right)\left(\overline{\mathrm{f}}_{\alpha_{1}} \overline{\mathrm{f}}^{\beta} \triangleright v\right)\right) \cdot\left(\overline{\mathrm{f}}_{\alpha_{2}} \overline{\mathrm{f}}_{\beta} \triangleright a\right) \\
& +\varepsilon\left(\overline{\mathrm{f}}^{\alpha}\right)\left(\overline{\mathrm{f}}_{\alpha_{1}} \overline{\mathrm{f}}^{\beta} \triangleright v\right) \otimes_{A} \mathrm{~d}\left(\overline{\mathrm{f}}_{\alpha_{2}} \overline{\mathrm{f}}_{\beta} \triangleright a\right) \\
= & \left(\left(\overline{\mathrm{f}}_{1}^{\alpha} \overline{\mathrm{f}}^{\beta} \triangleright \nabla\right)\left(\overline{\mathrm{f}}_{2}^{\alpha} \overline{\mathrm{f}}_{\beta} \triangleright v\right)\right) \cdot\left(\left(\overline{\mathrm{f}}_{\alpha} \triangleright a\right)+\left(\overline{\mathrm{f}}^{\beta} \triangleright v\right) \otimes_{A} \mathrm{~d}\left(\overline{\mathrm{f}}_{\beta} \triangleright a\right)\right. \\
= & D_{\mathcal{F}}(\nabla)(v) \star a+\left(\overline{\mathrm{f}}^{\beta} \triangleright v\right) \otimes_{A}\left(\overline{\mathrm{f}}_{\beta} \triangleright \mathrm{d} a\right) .
\end{aligned}
$$

In the second line we have used (6.8), in the third line the twist cocycle property (3.4c) and in the last line (6.6) and (6.7). Applying $\varphi^{-1}$ we obtain

$$
\widetilde{D}_{\mathcal{F}}(\nabla)(v \star a)=\widetilde{D}_{\mathcal{F}}(\nabla)(v) \star a+v \otimes_{A_{\star}} \mathrm{d} a
$$

The property $\widetilde{D}_{\mathcal{F}}^{-1}\left(\nabla_{\star}\right) \in \operatorname{Con}_{A}(V)_{\star}$, for all $\nabla_{\star} \in \operatorname{Con}_{A_{\star}}\left(V_{\star}\right)$, follows from twisting back $H^{\mathcal{F}}$ and all its modules to the original undeformed structures via the twist $\mathcal{F}^{-1}$.

Finally $\widetilde{D}_{\mathcal{F}}$ is an affine space isomorphism because its $\mathbb{K}$-linearity implies that, for all $\nabla \in \operatorname{Con}_{A}(V)_{\star}, P \in \operatorname{Hom}_{A}\left(V, V \otimes_{A} \Omega\right)_{\star}, \widetilde{D}_{\mathcal{F}}(\nabla+P)=\widetilde{D}_{\mathcal{F}}(\nabla)$ $+\widetilde{D}_{\mathcal{F}}(P) \in \operatorname{Con}_{A_{\star}}\left(V_{\star}\right)$.

If we forget the ${ }^{H} \mathscr{M}$-module and ${ }^{H^{\mathcal{F}}} \mathscr{M}$-module structures, the $\mathbb{K}$-modules $\operatorname{Hom}_{A}\left(V, V \otimes_{A} \Omega\right)$ and $\operatorname{Hom}_{A}\left(V, V \otimes_{A} \Omega\right)_{\star}$ coincide, and hence $\operatorname{Con}_{A}(V)$ and $\operatorname{Con}_{A}(V)_{\star}$ coincide as affine spaces. Theorem 6.5 then implies the isomorphism $\operatorname{Con}_{A}(V) \simeq \operatorname{Con}_{A_{\star}}\left(V_{\star}\right)$ between the affine space of connections $\operatorname{Con}_{A}(V)$ over the $\mathbb{K}$-module $\operatorname{Hom}_{A}\left(V, V \otimes_{A} \Omega\right)$ and the affine space of connections $\operatorname{Con}_{A_{\star}}\left(V_{\star}\right)$ over the $\mathbb{K}$-module $\operatorname{Hom}_{A_{\star}}\left(V_{\star}, V_{\star} \otimes_{A_{\star}} \Omega_{\star}\right)$.

Left modules. As in Theorem 6.5 we have an isomorphism ${ }_{A} \operatorname{Con}(V) \simeq$ $A_{\star} \operatorname{Con}\left(V_{\star}\right)$ between the affine spaces of connections on an ${ }^{H}{ }_{A} \mathscr{M}$-module $V$ and on the deformed ${ }^{H^{\mathcal{F}}} \mathscr{A} \mathscr{M}$-module $V_{\star}$. In this case we consider the $H_{A_{\star}}^{\mathcal{F}} \mathscr{M}$-module isomorphism $\varphi^{-1}:\left(\Omega \otimes_{A} V\right)_{\star} \longrightarrow \Omega_{\star} \otimes_{A_{\star}} V_{\star}$ and its lift $\varphi^{-1}:\left(\operatorname{Hom}_{\mathbb{K}}\left(V_{\star},\left(\Omega \otimes_{A} V\right)_{\star}\right)\right)^{o p} \longrightarrow\left(\operatorname{Hom}_{\mathbb{K}}\left(V_{\star}, \Omega_{\star} \otimes_{A_{\star}} V_{\star}\right)\right)^{o p}$. Using also the $D_{\mathcal{F}}^{c o p}$ isomorphism of Theorem 4.8 and denoting by ${ }_{A} \operatorname{Con}(V)_{\star}$ the set of connections ${ }_{A} \operatorname{Con}_{A}(V)$ when seen as an affine space over the ${ }^{H^{\mathcal{F}}} \mathscr{M}$-module $\left({ }_{A} \operatorname{Hom}\left(V, V \otimes_{A} \Omega\right)\right)_{\star}^{o p}$, rather than over the ${ }^{H} \mathscr{M}$-module $\left({ }_{A} \operatorname{Hom}\left(V, V \otimes_{A}\right.\right.$ $\Omega))^{o p}$, we obtain 
Theorem 6.6. Let $H$ be a Hopf algebra with twist $\mathcal{F} \in H \otimes H, A$ be an ${ }^{H} \mathscr{A}$-algebra, $V$ be an ${ }^{H}{ }_{A} \mathscr{M}$-module and $\left(\Omega^{\bullet}, \wedge, \mathrm{d}\right)$ be a left $H$-covariant differential calculus over $A$. Then the ${ }^{H^{\mathcal{F}}} \mathscr{M}$-module isomorphism

$$
\begin{aligned}
\widetilde{D}_{\mathcal{F}}^{c o p}:=\varphi^{-1} \circ D_{\mathcal{F}}^{c o p}:\left(\operatorname{Hom}_{\mathbb{K}}\left(V, \Omega \otimes_{A} V\right)\right)^{o p} & \rightarrow\left(\operatorname{Hom}_{\mathbb{K}}\left(V_{\star}, \Omega_{\star} \otimes_{A_{\star}} V_{\star}\right)\right)^{o p}, \\
(6.18) & P \mapsto \varphi^{-1} \circ\left(\overline{\mathrm{f}}_{\alpha}{ }^{c o p} P\right) \circ \overline{\mathrm{f}}^{\alpha}{ }^{\alpha}
\end{aligned}
$$

restricts to the ${ }^{H^{\mathcal{F}}} \mathscr{M}$-module isomorphism

$$
\widetilde{D}_{\mathcal{F}}^{\text {cop }}:\left({ }_{A} \operatorname{Hom}\left(V, \Omega \otimes_{A} V\right)\right)_{\star}^{o p} \longrightarrow\left({ }_{A_{\star}} \operatorname{Hom}\left(V_{\star}, \Omega_{\star} \otimes_{A_{\star}} V_{\star}\right)\right)^{o p}
$$

and to the affine space isomorphism

$$
\widetilde{D}_{\mathcal{F}}^{c o p}:{ }_{A} \operatorname{Con}(V)_{\star} \longrightarrow{ }_{A_{\star}} \operatorname{Con}\left(V_{\star}\right),
$$

where ${ }_{A} \operatorname{Con}(V)_{\star}$ and ${ }_{A_{\star}} \operatorname{Con}\left(V_{\star}\right)$ are affine spaces over the isomorphic ${ }^{H^{\mathcal{F}}} \mathscr{M}$ modules $\left({ }_{A} \operatorname{Hom}\left(V, \Omega \otimes_{A} V\right)\right)_{\star}^{o p}$ and $\left({ }_{A_{\star}} \operatorname{Hom}\left(V_{\star}, \Omega_{\star} \otimes_{A_{\star}} V_{\star}\right)\right)^{o p}$.

Bimodules. Let $V$ be an ${ }_{A} \mathscr{M}_{A}$-module, then $\operatorname{Hom}_{A}\left(V, V \otimes_{A} \Omega\right)$ and $\left({ }_{A} \operatorname{Hom}\left(V, \Omega \otimes_{A} V\right)\right)^{o p}$ are ${ }_{A} \mathscr{M}_{A^{-}}$-modules. Consequently, $\mathrm{Con}_{A}(V)_{\star}$, $\operatorname{Con}_{A_{\star}}\left(V_{\star}\right),{ }_{A} \operatorname{Con}(V)_{\star}$ and ${ }_{A_{\star}} \operatorname{Con}\left(V_{\star}\right)$ are affine spaces over the ${ }_{A_{\star} \mathcal{F}_{\star}} \mathscr{M}_{A_{\star}-}$ modules $\operatorname{Hom}_{A}\left(V, V \otimes_{A} \Omega\right)_{\star}, \operatorname{Hom}_{A_{\star}}\left(V_{\star}, V_{\star} \otimes_{A_{\star}} \Omega_{\star}\right),\left({ }_{A} \operatorname{Hom}\left(V, \Omega \otimes_{A} V\right)\right)_{\star}^{o p}$ and $\left({ }_{A_{\star}} \operatorname{Hom}\left(V_{\star}, \Omega_{\star} \otimes_{A_{\star}} V_{\star}\right)\right)^{o p}$, respectively. The isomorphisms (6.12) and (6.19) are ${ }^{H^{\mathcal{F}}}{ }_{A_{\star}} \mathscr{M}_{A_{\star}}$-module isomorphisms, and hence $\widetilde{D}_{\mathcal{F}}: \operatorname{Con}_{A}(V)_{\star} \rightarrow$ $\operatorname{Con}_{A_{\star}}\left(V_{\star}\right)$ (cf. Theorem 6.5) and $\widetilde{D}_{\mathcal{F}}^{\text {cop }}:{ }_{A} \operatorname{Con}(V)_{\star} \rightarrow{ }_{A_{\star}} \operatorname{Con}\left(V_{\star}\right)$ (cf. Theorem 6.6) are affine space isomorphisms compatible with the ${ }_{H^{\mathcal{F}}}{ }_{A_{\star}} \mathscr{M}_{A_{\star}-}$ module structures.

\subsection{Connections on tensor product modules (sum of connections)}

We now investigate the extension of connections to tensor product modules. We mention that this is relevant in the formulation of noncommutative gravity and gauge theories, since it provides a construction principle for connections on deformed tensor fields in terms of a fundamental connection on deformed vector fields, and a minimal coupling prescription for noncommutative fields with different charges.

The other ingredient in order to construct connections on arbitrary covariant and contravariant tensor fields is the extension of a connection on a module to the dual module. This will be discussed later in Subsection 6.5. 
Let $(H, \mathcal{R})$ be a quasitriangular Hopf algebra. A left $H$-covariant differential calculus $\left(\Omega^{\bullet}, \wedge, \mathrm{d}\right)$ over an ${ }^{H} \mathscr{A}$-algebra $A$ is called graded quasicommutative if the ${ }^{H} \mathscr{A}$-algebra $\Omega^{\bullet}$ is graded quasi-commutative, i.e., for all $\omega, \omega^{\prime} \in \Omega^{\bullet}$ of homogeneous degree,

$$
\omega \wedge \omega^{\prime}=(-1)^{\operatorname{deg}(\omega) \operatorname{deg}\left(\omega^{\prime}\right)}\left(\bar{R}^{\alpha} \triangleright \omega^{\prime}\right) \wedge\left(\bar{R}_{\alpha} \triangleright \omega\right) .
$$

Notice that graded quasi-commutativity of the algebra $\left(\Omega^{\bullet}, \wedge\right)$ in particular implies quasi-commutativity of the ${ }^{H} \mathscr{A}$-algebra $A$ and strong quasicommutativity of the ${ }^{H}{ }_{A} \mathscr{M}_{A}$-module of one-forms $\Omega$ (and also of all other ${ }^{H} \mathscr{M}_{A}$-modules of $n$-forms $\Omega^{n}, n \geq 1$ ).

Vice versa, it can be shown that a left $H$-covariant differential calculus $\left(\Omega^{\bullet}, \wedge, \mathrm{d}\right)$ over a quasi-commutative ${ }^{H} \mathscr{A}$-algebra $A$ is graded quasicommutative if the ${ }_{A}{ }_{A} \mathscr{M}_{A}$-module of one-forms $\Omega$ is quasi-commutative and generates $\Omega^{n}$ for all $n>1 .^{1}$

Examples of graded quasi-commutative left $H$-covariant differential calculi are presented in Example 6.2.

Lemma 6.7. Let $A$ be a quasi-commutative ${ }^{H} \mathscr{A}$-algebra, $W$ be a quasicommutative ${ }_{A}{ }_{A} \mathscr{M}_{A}$-module and $\Omega$ be a strong quasi-commutative ${ }_{A}{ }_{A} \mathscr{M}_{A^{-}}$ module. Then the braiding map $\tau_{\mathcal{R}}: W \otimes \Omega \rightarrow \Omega \otimes W$ (defined in (5.8a)) canonically induces an ${ }_{A} \mathscr{M}_{A}$-module isomorphism on the quotient (that with slight abuse of notation we still call $\tau_{\mathcal{R}}$ ),

$$
\tau_{\mathcal{R}}: W \otimes_{A} \Omega \longrightarrow \Omega \otimes_{A} W .
$$

Explicitly, for all $w \in W$ and $\theta \in \Omega$,

$$
\tau_{\mathcal{R}}\left(w \otimes_{A} \theta\right)=\left(\bar{R}^{\alpha} \triangleright \theta\right) \otimes_{A}\left(\bar{R}_{\alpha} \triangleright w\right) .
$$

We call the isomorphism $\tau_{\mathcal{R}}$ a braiding map because it braids the strong quasi-commutative ${ }^{H}{ }_{A} \mathscr{M}_{A}$-module $\Omega$ with the quasi-commutative ${ }^{{ }_{A}} \mathscr{M}_{A^{-}}$ modules $V, W$,

$$
\tau_{\mathcal{R} V \otimes_{A} W, \Omega}=\left(\tau_{\mathcal{R} V, \Omega} \otimes_{\mathcal{R}} \operatorname{id}_{W}\right) \circ\left(\operatorname{id}_{V} \otimes_{\mathcal{R}} \tau_{\mathcal{R} W, \Omega}\right) .
$$

\footnotetext{
${ }^{1}$ Hint: 1) Show that $\Omega$ is strong quasi-commutative. 2) Show that (6.21) holds for $\omega=\mathrm{d} a$ and $\omega^{\prime}=\mathrm{d} b$ with $a, b \in A$, then extend the result to arbitrary $\omega, \omega^{\prime} \in \Omega$. 3) Show that if $(6.21)$ holds for $\omega, \omega^{\prime} \in \Omega^{\bullet}$ with $\operatorname{deg}(\omega), \operatorname{deg}\left(\omega^{\prime}\right) \leq n$, then it also holds for $\omega, \omega^{\prime} \in \Omega^{\bullet}$ with $\operatorname{deg}(\omega), \operatorname{deg}\left(\omega^{\prime}\right) \leq n+1$.
} 
Furthermore,

$$
\tau_{\mathcal{R} V, W \otimes_{A} \Omega}=\left(\operatorname{id}_{W} \otimes_{\mathcal{R}} \tau_{\mathcal{R}} V, \Omega\right) \circ\left(\tau_{\mathcal{R} V, W} \otimes_{\mathcal{R}} \operatorname{id}_{\Omega}\right)
$$

if also the ${ }^{H}{ }_{A} \mathscr{M}_{A}$-module $W$ is strong quasi-commutative.

Proof. Since $\tau_{\mathcal{R}}: W \otimes \Omega \rightarrow \Omega \otimes W$ satisfies $\tau_{\mathcal{R}}\left(\mathcal{N}_{W, \Omega}\right) \subseteq \mathcal{N}_{\Omega, W}$ the induced map $\tau_{\mathcal{R}}: W \otimes_{A} \Omega \rightarrow \Omega \otimes_{A} W$ is well-defined. Since $\tau_{\mathcal{R}}: W \otimes \Omega \rightarrow \Omega \otimes W$ is an ${ }^{H} \mathscr{M}$-module homomorphism and $\mathcal{N}_{W, \Omega} \subset W \otimes \Omega, \mathcal{N}_{\Omega, W} \subset \Omega \otimes W$ are ${ }^{H} \mathscr{M}$-submodules, also the induced map $\tau_{\mathcal{R}}: W \otimes_{A} \Omega \rightarrow \Omega \otimes_{A} W$ is an ${ }^{H} \mathscr{M}$ module homomorphism. It is an ${ }^{H} \mathscr{M}_{A}$-module homomorphism as it can be easily checked using (6.23), quasi-commutativity of $W$ and strong quasicommutativity of $\Omega$.

Consider now the map $\tau_{\mathcal{R}}^{-1}: \Omega \otimes_{A} W \rightarrow W \otimes_{A} \Omega$, which is canonically induced by $\tau_{\mathcal{R}}^{-1}: \Omega \otimes W \rightarrow W \otimes \Omega$. Explicitly, for all $w \in W$ and $\theta \in \Omega$, $\tau_{\mathcal{R}}^{-1}\left(\theta \otimes_{A} w\right)=\left(R_{\alpha} \triangleright w\right) \otimes_{A}\left(R^{\alpha} \triangleright \theta\right)$. With a similar argument as above, one shows that $\tau_{\mathcal{R}}^{-1}$ is a well-defined ${ }_{A} \mathscr{M}_{A}$-module homomorphism. The easily proven equalities $\tau_{\mathcal{R}} \circ \tau_{\mathcal{R}}^{-1}=\mathrm{id}_{\Omega \otimes_{A} W}$ and $\tau_{\mathcal{R}}^{-1} \circ \tau_{\mathcal{R}}=\mathrm{id}_{W \otimes_{A} \Omega}$ show that $\tau_{\mathcal{R}}: W \otimes_{A} \Omega \rightarrow \Omega \otimes_{A} W$ is an ${ }_{A} \mathscr{M}_{A}$-module isomorphism.

The braiding properties (6.24) and (6.25) can be written with the $\mathcal{R}$ tensor product $\otimes_{\mathcal{R}}$ replaced by the usual tensor product because all maps are $H$-equivariant. Then these properties follow from the $\mathcal{R}$-matrix properties (5.3).

Proposition 6.8. Let $(H, \mathcal{R})$ be a quasitriangular Hopf algebra, $A$ be a quasi-commutative ${ }^{H} \mathscr{A}$-algebra, $W$ be a quasi-commutative ${ }^{H}{ }_{A} \mathscr{M}_{A}$-module and $\left(\Omega^{\bullet}, \wedge, \mathrm{d}\right)$ be a graded quasi-commutative left $H$-covariant differential calculus over $A$. Then any right connection $\nabla \in \operatorname{Con}_{A}(V)$ is also a quasileft connection in the sense that we have the braided left Leibniz rule, for all $a \in A$ and $w \in W$,

$$
\nabla(a \cdot w)=\left(\bar{R}^{\alpha} \triangleright a\right) \cdot\left(\bar{R}_{\alpha} \triangleright \nabla\right)(w)+\left(R_{\alpha} \triangleright w\right) \otimes_{A}\left(R^{\alpha} \triangleright \mathrm{d} a\right)
$$

More generally, for all $\xi \in H, a \in A$ and $w \in W$,

$$
\begin{aligned}
& (\xi \triangleright \nabla)(a \cdot w) \\
= & \left(\bar{R}^{\alpha} \triangleright a\right) \cdot\left(\bar{R}_{\alpha} \xi \triangleright \nabla\right)(w)+\varepsilon(\xi)\left(R_{\alpha} \triangleright w\right) \otimes_{A}\left(R^{\alpha} \triangleright \mathrm{d} a\right) .
\end{aligned}
$$


Proof. For all $a \in A$ and $w \in W$,

$$
\begin{aligned}
\nabla(a \cdot w) & =\nabla\left(\left(R_{\alpha} \triangleright w\right) \cdot\left(R^{\alpha} \triangleright a\right)\right) \\
& =\nabla\left(R_{\alpha} \triangleright w\right) \cdot\left(R^{\alpha} \triangleright a\right)+R_{\alpha} \triangleright w \otimes_{A} \mathrm{~d}\left(R^{\alpha} \triangleright a\right) \\
& =\left(\bar{R}^{\beta} R^{\alpha} \triangleright a\right) \cdot \bar{R}_{\beta} \triangleright\left(\nabla\left(R_{\alpha} \triangleright w\right)\right)+R_{\alpha} \triangleright w \otimes_{A} R^{\alpha} \triangleright \mathrm{d} a \\
& =\left(\bar{R}^{\beta} \bar{R}^{\alpha} \triangleright a\right) \cdot \bar{R}_{\beta} \triangleright\left(\nabla\left(S\left(\bar{R}_{\alpha}\right) \triangleright w\right)\right)+R_{\alpha} \triangleright w \otimes_{A} R^{\alpha} \triangleright \mathrm{d} a \\
& =\left(\bar{R}^{\beta} \triangleright a\right) \cdot\left(\bar{R}_{\beta} \triangleright \nabla\right)(w)+R_{\alpha} \triangleright w \otimes_{A} R^{\alpha} \triangleright \mathrm{d} a,
\end{aligned}
$$

where in the second line we have used that the ${ }^{H}{ }_{A} \mathscr{M}_{A}$-module $W \otimes_{A} \Omega$ is quasi-commutative (since both $W$ and $\Omega$ are, cf. Proposition 5.13), and in the third line the property $(5.7 \mathrm{~b})$ of the $\mathcal{R}$-matrix.

The more general expression for $\xi \triangleright \nabla$ follows from (6.8).

Theorem 6.9. Let $(H, \mathcal{R})$ be a quasitriangular Hopf algebra, A be a quasicommutative ${ }^{H} \mathscr{A}$-algebra, $W$ be a quasi-commutative ${ }^{{ }_{A}} \mathscr{M}_{A}$-module and $\left(\Omega^{\bullet}, \wedge, \mathrm{d}\right)$ be a graded quasi-commutative left $H$-covariant differential calculus over $A$. For any ${ }^{H} \mathscr{M}_{A}$-module $V$ and any $\nabla_{V} \in \operatorname{Con}_{A}(V), \nabla_{W} \in$ $\operatorname{Con}_{A}(W)$ we consider the $\mathbb{K}$-linear map $\nabla_{V} \widehat{\oplus}_{\mathcal{R}} \nabla_{W}: V \otimes W \rightarrow V \otimes_{A} W$ $\otimes_{A} \Omega$ defined by

$$
\nabla_{V} \widehat{\oplus}_{\mathcal{R}} \nabla_{W}:=\tau_{\mathcal{R} 23}^{-1} \circ \pi \circ\left(\nabla_{V} \otimes_{\mathcal{R}} \mathrm{id}\right)+\pi \circ\left(\mathrm{id} \otimes_{\mathcal{R}} \nabla_{W}\right)
$$

where the $\mathcal{R}$-tensor product of $\mathbb{K}$-linear maps was defined in (5.14), and where $\tau_{\mathcal{R} 23}^{-1}=\mathrm{id} \otimes_{\mathcal{R}} \tau_{\mathcal{R}}^{-1}$ is the inverse braiding map acting on the second and third entry of the tensor product $V \otimes_{A} \Omega \otimes_{A} W$ (the $\mathcal{R}$-tensor product in id $\otimes_{\mathcal{R}} \tau_{\mathcal{R}}^{-1}$ is defined in Theorem 5.16).

This map induces a connection on the quotient module $V \otimes_{A} W$,

$$
\nabla_{V} \oplus_{\mathcal{R}} \nabla_{W}: V \otimes_{A} W \rightarrow V \otimes_{A} W \otimes_{A} \Omega
$$

defined by, for all $v \in V, w \in W,\left(\nabla_{V} \oplus_{\mathcal{R}} \nabla_{W}\right)\left(v \otimes_{A} w\right):=\left(\nabla_{V} \widehat{\oplus}_{\mathcal{R}} \nabla_{W}\right)(v$ $\otimes w)$, i.e.,

$$
\begin{aligned}
& \left(\nabla_{V} \oplus_{\mathcal{R}} \nabla_{W}\right)\left(v \otimes_{A} w\right) \\
= & \tau_{\mathcal{R} 23}^{-1}\left(\nabla_{V}(v) \otimes_{A} w\right)+\left(\bar{R}^{\beta} \triangleright v\right) \otimes_{A}\left(\bar{R}_{\beta} \triangleright \nabla_{W}\right)(w),
\end{aligned}
$$

and extended by $\mathbb{K}$-linearity to all $V \otimes_{A} W$. 
Proof. The definition of $\nabla_{V} \widehat{\oplus}_{\mathcal{R}} \nabla_{W}$ slightly simplifies if we observe that $\nabla_{V} \otimes_{\mathcal{R}}$ id $=\nabla_{V} \otimes$ id, id $\otimes_{\mathcal{R}} \tau_{\mathcal{R}}^{-1}=\mathrm{id} \otimes \tau_{\mathcal{R}}^{-1}$, since id and $\tau_{\mathcal{R}}^{-1}$ are $H$-equivariant. We first prove that the $\mathbb{K}$-linear map $\nabla_{V} \oplus_{\mathcal{R}} \nabla_{W}$ is well-defined by showing that $\operatorname{ker}(\pi) \subseteq \operatorname{ker}\left(\nabla_{\mathrm{V}} \widehat{\oplus}_{\mathcal{R}} \nabla_{\mathrm{W}}\right)$. In order to simplify the notation in the proof we denote both connections $\nabla_{V}$ and $\nabla_{W}$ by $\nabla$. For all $v \in V$, $w \in W$ and $a \in A$,

$$
\begin{aligned}
\nabla \widehat{\oplus}_{\mathcal{R}} \nabla(v \cdot a \otimes w)= & \tau_{\mathcal{R} 23}^{-1}\left(\nabla(v \cdot a) \otimes_{A} w\right)+\left(\bar{R}^{\beta} \triangleright(v \cdot a)\right) \otimes_{A}\left(\bar{R}_{\beta} \triangleright \nabla\right)(w) \\
= & \tau_{\mathcal{R} 23}^{-1}\left((\nabla v) \otimes_{A} a \cdot w+v \otimes_{A} \mathrm{~d} a \otimes_{A} w\right) \\
& \quad \quad \quad\left(\bar{R}^{\beta} \triangleright v\right) \otimes_{A}\left(\bar{R}^{\alpha} \triangleright a\right) \cdot\left(\bar{R}_{\alpha} \bar{R}_{\beta} \triangleright \nabla\right)(w) \\
= & \tau_{\mathcal{R} 23}^{-1}\left((\nabla v) \otimes_{A} a \cdot w\right)+\left(\bar{R}^{\alpha} \triangleright v\right) \otimes_{A}\left(\bar{R}_{\alpha} \triangleright \nabla\right)(a \cdot w) \\
= & \nabla \widehat{\oplus}_{\mathcal{R}} \nabla(v \otimes a \cdot w),
\end{aligned}
$$

where in the second line we used the right Leibniz rule (6.3) and the property $(5.5 \mathrm{~b})$ of the $\mathcal{R}$-matrix. In the third line we used Proposition 6.8 and the normalization property of the $\mathcal{R}$-matrix (5.7a).

The $\mathbb{K}$-linear map $\nabla_{V} \oplus_{\mathcal{R}} \nabla_{W}$ defined in (6.30) is a connection because it satisfies the right Leibniz rule (6.3), for all $v \in V, w \in W$ and $a \in A$,

$$
\begin{aligned}
\left(\nabla \oplus_{\mathcal{R}} \nabla\right) & \left(v \otimes_{A} w \cdot a\right) \\
& =\tau_{\mathcal{R} 23}^{-1}\left((\nabla v) \otimes_{A} w \cdot a\right)+\left(\bar{R}^{\alpha} \triangleright v\right) \otimes_{A}\left(\bar{R}_{\alpha} \nabla \nabla\right)(w \cdot a) \\
& =\left(\nabla \oplus_{\mathcal{R}} \nabla\right)\left(v \otimes_{A} w\right) \cdot a+\varepsilon\left(\bar{R}_{\alpha}\right)\left(\bar{R}^{\alpha} \triangleright v\right) \otimes_{A} w \otimes_{A} \mathrm{~d} a \\
& =\left(\nabla \oplus_{\mathcal{R}} \nabla\right)\left(v \otimes_{A} w\right) \cdot a+v \otimes_{A} w \otimes_{A} \mathrm{~d} a,
\end{aligned}
$$

where in the second line we have used that $\tau_{\mathcal{R}}$ is an ${ }^{H} \mathscr{M}_{A}$-module isomorphism and (6.8), and in the last line the normalization property of the $\mathcal{R}$-matrix (5.7a).

The sum of connections is compatible with the Hopf algebra action, for all $\xi \in H$,

$$
\xi \triangleright\left(\nabla_{V} \oplus_{\mathcal{R}} \nabla_{W}\right)=\left(\xi \triangleright \nabla_{V}\right) \oplus_{\mathcal{R}}\left(\xi \triangleright \nabla_{W}\right)
$$

This property easily follows recalling property (5.18a) and observing that all the maps in (6.29), but the connections $\nabla_{V}$ and $\nabla_{W}$, are $H$-equivariant.

We end this subsection showing that the sum of connections is associative.

Theorem 6.10. Let $(H, \mathcal{R})$ be a quasitriangular Hopf algebra, A be a quasicommutative ${ }^{H} \mathscr{A}$-algebra, $W, Z$ be two quasi-commutative ${ }^{{ }^{H}}{ }_{A} \mathscr{M}_{A}$-modules 
and $\left(\Omega^{\bullet}, \wedge, \mathrm{d}\right)$ be a graded quasi-commutative left $H$-covariant differential calculus over $A$. Then for any ${ }^{H} \mathscr{M}_{A}$-module $V, \nabla_{V} \in \operatorname{Con}_{A}(V), \nabla_{W} \in$ $\operatorname{Con}_{A}(W)$ and $\nabla_{Z} \in \operatorname{Con}_{A}(Z)$,

$$
\left(\nabla_{V} \oplus_{\mathcal{R}} \nabla_{W}\right) \oplus_{\mathcal{R}} \nabla_{Z}=\nabla_{V} \oplus_{\mathcal{R}}\left(\nabla_{W} \oplus_{\mathcal{R}} \nabla_{Z}\right)
$$

Proof. It is convenient to denote by $\tau_{\mathcal{R} i}^{-1}{ }_{i+1}=\mathrm{id} \otimes \cdots \otimes \tau_{\mathcal{R}}^{-1} \otimes \cdots \otimes \mathrm{id}$ the inverse braiding map acting on the $i$-th and $(i+1)$-th entry of a tensor product. The inverse braiding map exchanging the $i$-th entry with the $(i+1)$-th and $(i+2)$-th entry is denoted by $\tau_{\mathcal{R} i(i+1 i+2)}^{-1}$ and similarly $\tau_{\mathcal{R}(i i+1) i+2}^{-1}$ is the inverse braiding map exchanging the $i$-th and $(i+1)$-th entry with the $(i+2)$-th entry. For example, $\tau_{\mathcal{R}(12) 3}^{-1}(a \otimes b \otimes c)=\left(R_{\alpha} \triangleright c\right) \otimes R^{\alpha} \triangleright(a \otimes b)$ and $\tau_{\mathcal{R} 1(23)}^{-1}(a \otimes b \otimes c)=R_{\alpha} \triangleright(b \otimes c) \otimes\left(R^{\alpha} \triangleright a\right)$.

Associativity of $\oplus_{\mathcal{R}}$ is implied by associativity of $\widehat{\oplus}_{\mathcal{R}}$. The latter follows from the associativity of $\otimes_{\mathcal{R}}$, the $H$-equivariance of the id, $\tau_{\mathcal{R}}^{-1}$ and $\pi$ maps, the composition property $(5.18 \mathrm{c})$, the composition of projections property $\pi \circ\left(\right.$ id $\left.\otimes_{\mathcal{R}} \pi\right)=\pi \circ\left(\pi \otimes_{\mathcal{R}} \mathrm{id}\right)$, and the braid relation (6.24).

We here present a less abstract proof by evaluating (6.35) on $V \otimes_{A}$ $W \otimes_{A} Z$. Due to $\mathbb{K}$-linearity it is enough to show associativity on elements $v \otimes_{A} w \otimes_{A} z \in V \otimes_{A} W \otimes_{A} Z$. We denote all connections by $\nabla$ in order to simplify the notation and find

$$
\begin{aligned}
\left(\left(\nabla \oplus_{\mathcal{R}} \nabla\right) \oplus_{\mathcal{R}} \nabla\right)\left(v \otimes_{A} \otimes w \otimes_{A} z\right) & \\
= & \tau_{\mathcal{R} 34}^{-1}\left(\left(\nabla \oplus_{\mathcal{R}} \nabla\right)\left(v \otimes_{A} w\right) \otimes_{A} z\right) \\
& +\bar{R}^{\alpha} \triangleright\left(v \otimes_{A} w\right) \otimes_{A}\left(\bar{R}_{\alpha} \triangleright \nabla\right)(z) \\
= & \tau_{\mathcal{R} 34}^{-1}\left(\tau_{\mathcal{R} 23}^{-1}\left(\nabla(v) \otimes_{A} w\right) \otimes_{A} z\right. \\
& \left.+\left(\bar{R}^{\alpha} \triangleright v\right) \otimes_{A}\left(\bar{R}_{\alpha} \triangleright \nabla\right)(w) \otimes_{A} z\right) \\
& +\left(\bar{R}^{\alpha} \triangleright v\right) \otimes_{A}\left(\bar{R}^{\beta} \triangleright w\right) \otimes_{A}\left(\bar{R}_{\beta} \bar{R}_{\alpha} \triangleright \nabla\right)(z) \\
= & \tau_{\mathcal{R} 34}^{-1}\left(\tau_{\mathcal{R} 23}^{-1}\left(\nabla(v) \otimes_{A} w \otimes_{A} z\right)\right) \\
& +\left(\bar{R}^{\alpha} \triangleright v\right) \otimes_{A} \tau_{\mathcal{R} 23}^{-1}\left(\left(\bar{R}_{\alpha} \triangleright \nabla\right)(w) \otimes_{A} z\right) \\
& +\left(\bar{R}^{\alpha} \triangleright v\right) \otimes_{A}\left(\bar{R}^{\beta} \triangleright w\right) \otimes_{A}\left(\bar{R}_{\beta} \bar{R}_{\alpha} \triangleright \nabla\right)(z) \\
= & \tau_{\mathcal{R} 2(34)}^{-1}\left(\nabla(v) \otimes_{A} w \otimes_{A} z\right) \\
& +\left(\bar{R}^{\alpha} \triangleright v\right) \otimes_{A}\left(\bar{R}_{a} \triangleright\left(\nabla \oplus_{\mathcal{R}} \nabla\right)\right)\left(w \otimes_{A} z\right) \\
= & \left(\nabla \oplus_{\mathcal{R}}\left(\nabla \oplus_{\mathcal{R}} \nabla\right)\right)\left(v \otimes_{A} \otimes_{A} w \otimes_{A} z\right)
\end{aligned}
$$

where in the fourth line we used $\tau_{\mathcal{R} 2(34)}^{-1}=\tau_{\mathcal{R} 34}^{-1} \circ \tau_{\mathcal{R} 23}^{-1}$, which follows from the braid relation (6.24), and property (6.34). 


\subsection{Deformation of the sum of connections}

We study the deformation of the sum of two connections. We need a preliminary

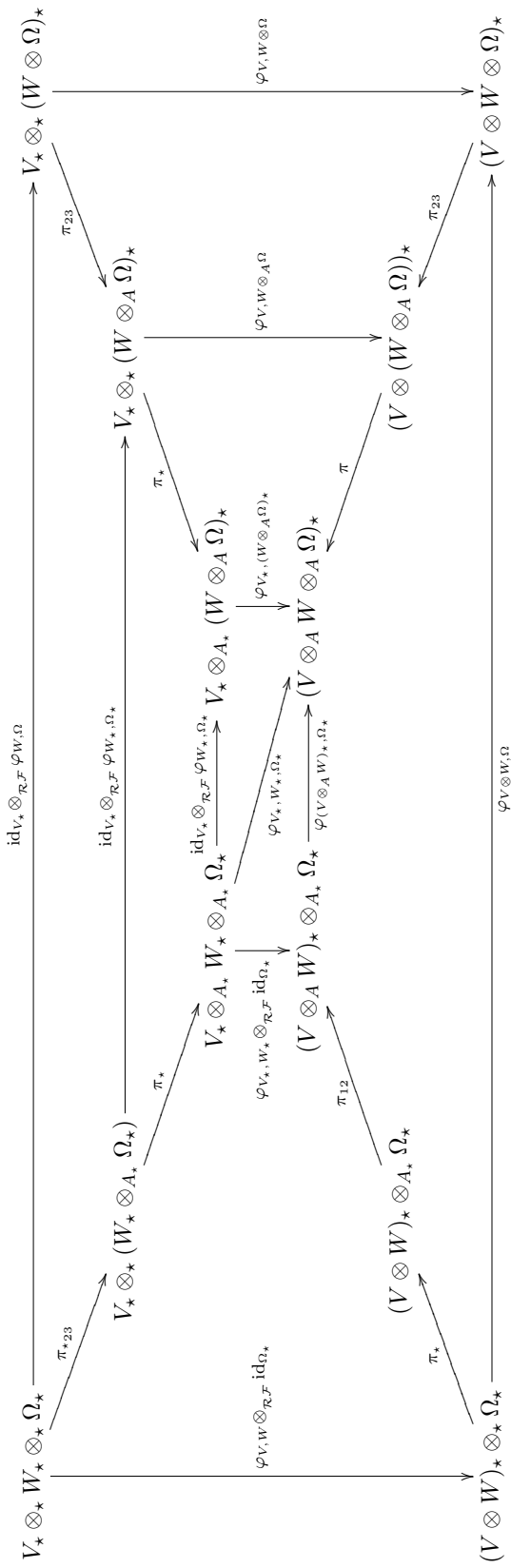


Lemma 6.11. Let $(H, \mathcal{R})$ be a quasitriangular Hopf algebra with twist $\mathcal{F} \in$

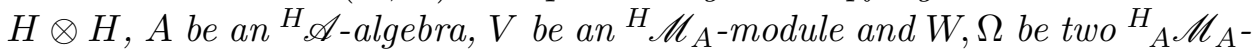
modules. The commutative diagram (5.30) induces the commutative diagram (6.37), where the inner diagonal map $\varphi_{V_{\star}, W_{\star}, \Omega_{\star}}: V_{\star} \otimes_{A_{\star}} W_{\star} \otimes_{A_{\star}} \Omega_{\star} \rightarrow$ $\left(V \otimes_{A} W \otimes_{A} \Omega\right)_{\star}$, defined as the composition $\varphi_{V_{\star}, W_{\star}, \Omega_{\star}}:=\varphi_{V_{\star},\left(W \otimes_{A} \Omega\right)_{\star}}$ $\circ\left(\operatorname{id}_{V_{\star}} \otimes_{\mathcal{R}^{\mathcal{F}}} \varphi_{W_{\star}, \Omega_{\star}}\right)$, is an ${ }^{H^{\mathcal{F}}} \mathscr{M}_{A_{\star}}$-module isomorphism.

Proof. The diagram (6.37) is composed of the commutative trapezia defining the induced maps $\varphi$ on the quotients. The outer rectangle is the commutative diagram (5.30); its commutativity implies that all subdiagrams of (6.37) are commutative. Since all maps in the diagram are ${ }^{H^{\mathcal{F}}} \mathscr{M}_{A_{\star}}$-module homomorphisms, so is $\varphi_{V_{\star}, W_{\star}, \Omega_{\star}}$. Since the $\varphi$-maps in the outer rectangle are isomorphisms, so are all the induced $\varphi$-maps, hence in particular $\varphi_{V_{\star}, W_{\star}, \Omega_{\star}}$.

The deformation of the sum of connections $(6.30), D_{\mathcal{F}}\left(\nabla_{V} \oplus_{\mathcal{R}} \nabla_{W}\right)$ : $\left(V \otimes_{A} W\right)_{\star} \rightarrow\left(V \otimes_{A} W \otimes_{A} \Omega\right)_{\star}$, where $D_{\mathcal{F}}$ is the deformation isomorphism discussed in Theorem 4.7 , is by construction a $\mathbb{K}$-linear map. We show that up to $\varphi$-isomorphisms it is a connection.

Theorem 6.12. Let $(H, \mathcal{R})$ be a quasitriangular Hopf algebra with twist $\mathcal{F} \in H \otimes H, A$ a quasi-commutative ${ }^{H} \mathscr{A}$-algebra, $W$ a quasi-commutative ${ }_{A} \mathscr{M}_{A}$-module and $\left(\Omega^{\bullet}, \wedge, \mathrm{d}\right)$ a graded quasi-commutative left $H$-covariant differential calculus over $A$. Then for any ${ }^{H} \mathscr{M}_{A}$-module $V, \nabla_{V} \in \operatorname{Con}_{A}(V)$ and $\nabla_{W} \in \operatorname{Con}_{A}(W)$ the following diagram commutes

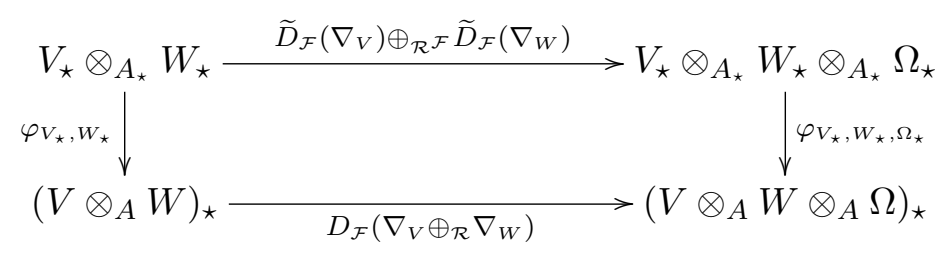

Proof. We recall that the sum of connections $\nabla_{V} \oplus_{\mathcal{R}} \nabla_{W}$ is canonically induced from the $\mathbb{K}$-linear map $\nabla_{V} \widehat{\oplus}_{\mathcal{R}} \nabla_{W}: V \otimes W \rightarrow V \otimes_{A} W \otimes_{A} \Omega$ defined in (6.29). We therefore first show commutativity of the following diagram

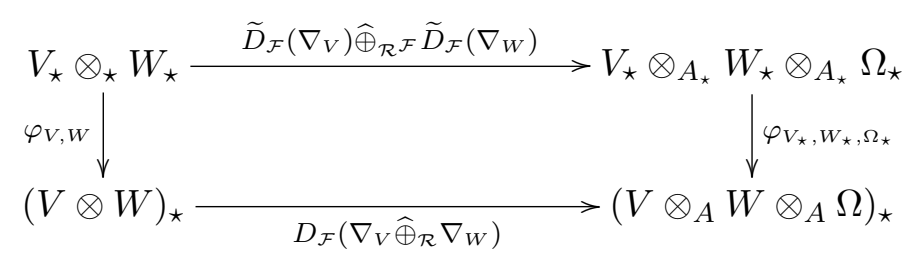


To simplify the notation we denote all connections by $\nabla$. Using $\mathbb{K}$-linearity of $D_{\mathcal{F}}$ and that $\tau_{\mathcal{R}}^{-1}$, id and $\pi$ are $H$-equivariant, we obtain

$$
\begin{aligned}
D_{\mathcal{F}}\left(\nabla \widehat{\oplus}_{\mathcal{R}} \nabla\right) & =D_{\mathcal{F}}\left(\tau_{\mathcal{R} 23}^{-1} \circ \pi \circ\left(\nabla \otimes_{\mathcal{R}} \mathrm{id}\right)+\pi \circ \mathrm{id} \otimes_{\mathcal{R}} \nabla\right) \\
& =\tau_{\mathcal{R} 23}^{-1} \circ \pi \circ D_{\mathcal{F}}\left(\nabla \otimes_{\mathcal{R}} \mathrm{id}\right)+\pi \circ D_{\mathcal{F}}\left(\mathrm{id} \otimes_{\mathcal{R}} \nabla\right) .
\end{aligned}
$$

Theorem 5.6 and $H$-equivariance of id implies

$$
\begin{aligned}
& D_{\mathcal{F}}\left(\nabla \widehat{\oplus}_{\mathcal{R}} \nabla\right) \\
& =\tau_{\mathcal{R} 23}^{-1} \circ \pi \circ \varphi_{V \otimes_{A} \Omega, W} \circ\left(D_{\mathcal{F}}(\nabla) \otimes_{\mathcal{R}^{\mathcal{F}}} \mathrm{id}\right) \circ \varphi_{V, W}^{-1} \\
& +\pi \circ \varphi_{V, W \otimes_{A} \Omega} \circ\left(\mathrm{id} \otimes_{\mathcal{R}^{\mathcal{F}}} D_{\mathcal{F}}(\nabla)\right) \circ \varphi_{V, W}^{-1} \\
& =\tau_{\mathcal{R} 23}^{-1} \circ \pi \circ \varphi_{V \otimes_{A} \Omega, W} \circ\left(\varphi_{V_{\star}, \Omega_{\star}} \otimes_{\mathcal{R}^{\mathcal{F}}} \mathrm{id}\right) \circ\left(\widetilde{D}_{\mathcal{F}}(\nabla) \otimes_{\mathcal{R}^{\mathcal{F}}} \mathrm{id}\right) \circ \varphi_{V, W}^{-1} \\
& +\pi \circ \varphi_{V, W \otimes_{A} \Omega} \circ\left(\mathrm{id} \otimes_{\mathcal{R}^{\mathcal{F}}} \varphi_{W_{\star}, \Omega_{\star}}\right) \circ\left(\mathrm{id} \otimes_{\mathcal{R}^{\mathcal{F}}} \widetilde{D}_{\mathcal{F}}(\nabla)\right) \circ \varphi_{V, W}^{-1} \\
& =\varphi_{V_{\star}, W_{\star}, \Omega_{\star}} \circ\left(\tau_{\mathcal{R}^{\mathcal{F}} 23}^{-1} \circ \pi_{\star} \circ\left(\widetilde{D}_{\mathcal{F}}(\nabla) \otimes_{\mathcal{R}^{\mathcal{F}}} \mathrm{id}\right)\right. \\
& \left.+\pi_{\star} \circ\left(\operatorname{id} \otimes_{\mathcal{R}^{\mathcal{F}}} \widetilde{D}_{\mathcal{F}}(\nabla)\right)\right) \circ \varphi_{V, W}^{-1},
\end{aligned}
$$

where in the last equality we have used: For the second addend commutativity of the sub-diagram

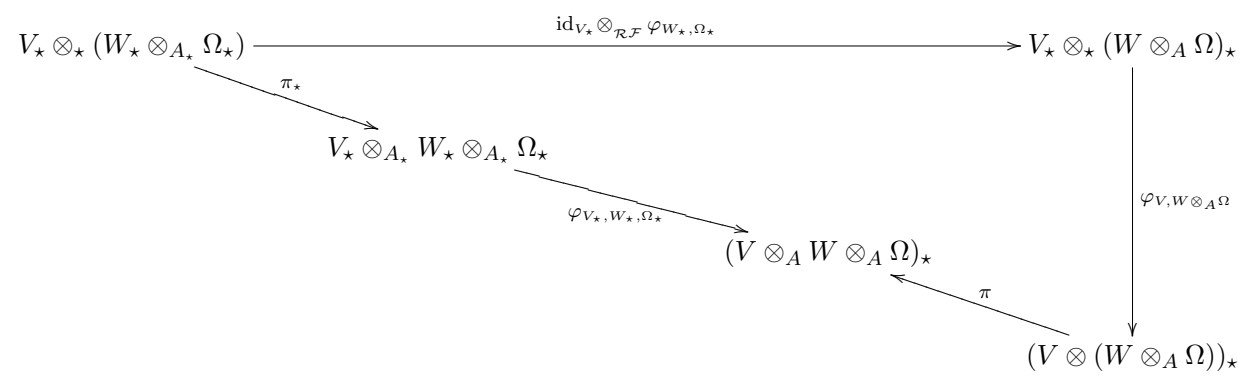

of the diagram (6.37). For the first addend we have used $\tau_{\mathcal{R}^{\mathcal{F}} 23}^{-1}=\left(\mathrm{id} \otimes_{\mathcal{R}^{\mathcal{F}}}\right.$ $\left.\varphi_{W_{\star}, \Omega_{\star}}^{-1}\right) \circ \tau_{\mathcal{R} 23}^{-1} \circ\left(\mathrm{id} \otimes_{\mathcal{R}^{\mathcal{F}}} \varphi_{\Omega_{\star}, W_{\star}}\right)$ (recall (5.39) and the text above (5.39)), $\varphi_{V_{\star},\left(W \otimes_{A} \Omega\right)_{\star}} \circ \tau_{\mathcal{R} 23}^{-1}=\tau_{\mathcal{R} 23}^{-1} \circ \varphi_{V_{\star},\left(\Omega \otimes_{A} W\right)_{\star}}$ (because of $H$-equivariance of $\left.\tau_{\mathcal{R} 23}^{-1}\right)$, and $\varphi_{V_{\star}, \Omega_{\star}, W_{\star}} \circ \pi_{\star}=\pi \circ \varphi_{V \otimes_{A} \Omega, W} \circ\left(\varphi_{V_{\star}, \Omega_{\star}} \otimes_{\mathcal{R}^{\mathcal{F}}}\right.$ id $)$. This last equality follows from a similar diagram as in Lemma 6.11. (Hint: start with the outer diagram drawn in (6.37) and use in the upper left corner the projection $\pi_{\star_{12}}$ instead of $\pi_{\star_{23}}$.)

Equation (6.41) shows commutativity of the diagram (6.39). Since the map $\varphi_{V, W}$ canonically induces on the quotient $V_{\star} \otimes_{A_{\star}} W_{\star}$ the map $\varphi_{V_{\star}, W_{\star}}$ (see $(5.51)$ ), and since the map $D_{\mathcal{F}}\left(\nabla \widehat{\oplus}_{\mathcal{R}} \nabla\right)$ canonically induces on the 
quotient $\left(V \otimes_{A} W\right)_{\star}$ the map $D_{\mathcal{F}}\left(\nabla \oplus_{\mathcal{R}} \nabla\right)$ (in fact, the actions $\triangleright$ and $\triangleright$ present in $D_{\mathcal{F}}$ are canonically induced on the quotient), the commutative diagram (6.39) induces the commutative diagram (6.38).

\subsection{From right to left connections}

Let $(H, \mathcal{R})$ be a quasitriangular Hopf algebra and $A$ be a quasi-commutative $H_{\mathscr{A}}$-algebra. We showed in Theorem 5.25 that the map $D_{\mathcal{R}}$, i.e. the deformation isomorphism with twist $\mathcal{F}=\mathcal{R}$, provides an isomorphism between right and left $A$-linear maps of strong quasi-commutative ${ }^{H}{ }_{A} \mathscr{M}_{A}$-modules. The aim of this subsection is to prove a similar statement for right and left connections on a strong quasi-commutative ${ }^{H}{ }_{A} \mathscr{M}_{A}$-module $V$.

We notice that due to Theorem 5.25 we have an ${ }^{H}{ }_{A} \mathscr{M}_{A}$-module isomorphism

$$
\begin{aligned}
D_{\mathcal{R}}: \operatorname{Hom}_{\mathbb{K}}\left(V, V \otimes_{A} \Omega\right) & \longrightarrow\left(\operatorname{Hom}_{\mathbb{K}}\left(V, V \otimes_{A} \Omega\right)\right)^{o p}, \\
P & \longmapsto D_{\mathcal{R}}(P)=\left(\bar{R}^{\alpha} \triangleright P\right) \circ \bar{R}_{\alpha} \triangleright
\end{aligned}
$$

between $\left(H_{\mathbb{K}}\left(V, V \otimes_{A} \Omega\right), \cdot, \boldsymbol{)}\right)$ and $\left(H_{\mathbb{K}}\left(V, V \otimes_{A} \Omega\right),{ }^{o p},{ }^{c o p}\right)$, which restricts to an ${ }_{A}^{H} \mathscr{M}_{A}$-module isomorphism (denoted by the same symbol)

$$
D_{\mathcal{R}}: \operatorname{Hom}_{A}\left(V, V \otimes_{A} \Omega\right) \rightarrow\left({ }_{A} \operatorname{Hom}\left(V, V \otimes_{A} \Omega\right)\right)^{o p}
$$

Composing this map with the inverse braiding map $\tau_{\mathcal{R}}^{-1}: V \otimes_{A} \Omega \rightarrow \Omega \otimes_{A} V$ we obtain another ${ }_{A}^{H} \mathscr{M}_{A}$-module isomorphism

$$
\begin{aligned}
\widetilde{D}_{\mathcal{R}}: \operatorname{Hom}_{\mathbb{K}}\left(V, V \otimes_{A} \Omega\right) & \longrightarrow\left(\operatorname{Hom}_{\mathbb{K}}\left(V, \Omega \otimes_{A} V\right)\right)^{o p} \\
P & \longmapsto \widetilde{D}_{\mathcal{R}}(P)=\tau_{\mathcal{R}}^{-1} \circ D_{\mathcal{R}}(P),
\end{aligned}
$$

which also restricts to an ${ }^{H} \mathscr{M}_{A}$-module isomorphism (denoted by the same symbol)

$$
\widetilde{D}_{\mathcal{R}}: \operatorname{Hom}_{A}\left(V, V \otimes_{A} \Omega\right) \rightarrow\left({ }_{A} \operatorname{Hom}\left(V, \Omega \otimes_{A} V\right)\right)^{o p}
$$

Theorem 6.13. Let $(H, \mathcal{R})$ be a quasitriangular Hopf algebra, $A$ be a quasicommutative ${ }^{H} \mathscr{A}$-algebra, $V$ be a strong quasi-commutative ${ }^{{ }^{H}} \mathscr{M}_{A}$-module and $\left(\Omega^{\bullet}, \wedge, \mathrm{d}\right)$ be a graded quasi-commutative left $H$-covariant differential calculus over $A$. Then the ${ }^{H}{ }_{A} \mathscr{M}_{A}$-module isomorphism (6.44) restricts to 
the affine space isomorphism

$$
\begin{aligned}
\widetilde{D}_{\mathcal{R}}: \operatorname{Con}_{A}(V) & \longrightarrow{ }_{A} \operatorname{Con}(V), \\
\nabla & \longmapsto \widetilde{D}_{\mathcal{R}}(\nabla)=\tau_{\mathcal{R}}^{-1} \circ\left(\bar{R}^{\alpha} \vee \nabla\right) \circ \bar{R}_{\alpha} \triangleright,
\end{aligned}
$$

where $\operatorname{Con}_{A}(V)$ and ${ }_{A} \operatorname{Con}(V)$ are respectively affine spaces over the isomorphic ${ }^{H} \mathscr{M}_{A}$-modules (cf. (6.45)) $\operatorname{Hom}_{A}\left(V, V \otimes_{A} \Omega\right)$ and $\left({ }_{A} \operatorname{Hom}\left(V, \Omega \otimes_{A}\right.\right.$ $V))^{o p}$.

Proof. By Lemma 5.23 we have that $A_{\star_{\mathcal{R}}}=A^{o p}, \Omega_{\star_{\mathcal{R}}}=\Omega^{o p}$ and $V_{\star_{\mathcal{R}}}=V^{o p}$. These equalities and the equality (6.16) (see Theorem 6.5) imply, for all $\nabla \in \operatorname{Con}_{A}(V), a \in A$ and $v \in V$,

$$
\begin{aligned}
D_{\mathcal{R}}(\nabla)(a \cdot v)=D_{\mathcal{R}}(\nabla)\left(v \star_{\mathcal{R}} a\right) & =D_{\mathcal{R}}(\nabla)(v) \star_{\mathcal{R}} a+\left(\bar{R}^{\alpha} \triangleright v\right) \otimes_{A}\left(\bar{R}_{\alpha} \triangleright \mathrm{d} a\right) \\
(6.47) & =a \cdot D_{\mathcal{R}}(\nabla)(v)+\left(\bar{R}^{\alpha} \triangleright v\right) \otimes_{A}\left(\bar{R}_{\alpha} \triangleright \mathrm{d} a\right) .
\end{aligned}
$$

Applying the ${ }_{A}^{H} \mathscr{M}_{A}$-module isomorphism $\tau_{\mathcal{R}}^{-1}$ we find

$$
\widetilde{D}_{\mathcal{R}}(\nabla)(a \cdot v)=a \cdot \widetilde{D}_{\mathcal{R}}(\nabla)(v)+\mathrm{d} a \otimes_{A} v .
$$

Thus $\widetilde{D}_{\mathcal{R}}(\nabla) \in{ }_{A} \operatorname{Con}(V)$ is a left connection on $V$. The map $\widetilde{D}_{\mathcal{R}}$ is invertible via

$$
\widetilde{D}_{\mathcal{R}}^{-1}:{ }_{A} \operatorname{Con}(V) \rightarrow \operatorname{Con}_{A}(V), \quad \nabla \mapsto \widetilde{D}_{\mathcal{R}}^{-1}(\nabla)=\tau_{\mathcal{R}} \circ\left(D_{\mathcal{R}}^{-1}(\nabla)\right),
$$

where $D_{\mathcal{R}}^{-1}(\nabla)=\left(R^{\alpha} \wedge^{c o p} \nabla\right) \circ R_{\alpha} \triangleright$, recall $\triangleright^{c o p}=\triangleright_{\mathcal{R}}$, and Remark 3.11.

Finally, $\widetilde{D}_{\mathcal{R}}$ is an affine space isomorphism because, for all $\nabla \in \operatorname{Con}_{A}(V)$ and $P \in \operatorname{Hom}_{A}\left(V, V \otimes_{A} \Omega\right), \widetilde{D}_{\mathcal{R}}(\nabla+P)=\widetilde{D}_{\mathcal{R}}(\nabla)+\widetilde{D}_{\mathcal{R}}(P)$, where $\widetilde{D}_{\mathcal{R}}(P)$ $\in\left({ }_{A} \operatorname{Hom}\left(V, \Omega \otimes_{A} V\right)\right)^{o p}$ is the image of the right $A$-linear map $P$ under the ${ }_{A} \mathscr{M}_{A}$-module isomorphism (6.45).

Remark 6.14. Similarly to Theorem 5.26 one can show that the right to left isomorphism for connections is compatible with twist deformation: Let $\mathcal{F} \in H \otimes H$ be a twist of the quasitriangular Hopf algebra $(H, \mathcal{R})$. Then, due to $H$-equivariance of the $\varphi$ and $\tau_{\mathcal{R}}$ maps, the following diagram of isomorphisms of affine spaces over ${ }^{H^{\mathcal{F}}}{ }_{A_{\star}} \mathscr{M}_{A_{\star}}$-modules commutes

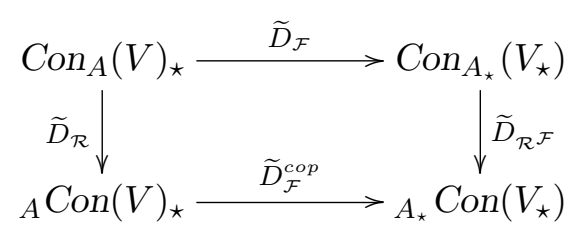




\subsection{Connections induced on dual modules}

A connection on a finitely generated and projective $\mathscr{M}_{A}$-module $V$ induces in a canonical way a connection on the dual ${ }_{A} \mathscr{M}$-module $V^{\prime}=\operatorname{Hom}_{A}(V, A)$. In the beginning of this subsection we review in detail this construction. It will be needed in the remaining part of the subsection, when we consider connections $\nabla$ on ${ }^{H} \mathscr{M}_{A}$-modules (and ${ }^{H}{ }_{A} \mathscr{M}_{A}$-modules), the associated dual connections $\nabla^{\prime}$, and their twist deformation.

There are various equivalent definitions of finitely generated and projective modules over a ring, see e.g. the monograph [22]. For our purposes it is enough to use their convenient characterization in terms of a pair of dual bases (this is the so-called Dual Basis Lemma).

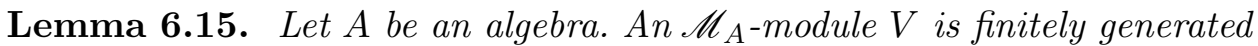
and projective if and only if there exists a family of elements $\left\{v_{i} \in V: i=\right.$ $1, \ldots, n\}$ and right $A$-linear maps $\left\{v_{i}^{\prime} \in V^{\prime}:=\operatorname{Hom}_{A}(V, A): i=1, \ldots, n\right\}$ with $n \in \mathbb{N}$, such that for any $v \in V$ we have

$$
v=\sum_{i=1}^{n} v_{i} \cdot v_{i}^{\prime}(v) .
$$

An $A \mathscr{M}$-module $V$ is finitely generated and projective if and only if there exists a family of elements $\left\{v_{i} \in V: i=1, \ldots, n\right\}$ and left A-linear maps $\left\{v_{i}^{\prime} \in V^{\prime}:={ }_{A} \operatorname{Hom}(V, A): i=1, \ldots, n\right\}$ with $n \in \mathbb{N}$, such that for any $v \in V$ we have

$$
v=\sum_{i=1}^{n} v_{i}^{\prime}(v) \cdot v_{i}
$$

The set $\left\{v_{i}, v_{i}^{\prime}: i=1, \ldots, n\right\}$ is loosely referred to "pair of dual bases" for $V$, even though $\left\{v_{i}\right\}$ is just a generating set of $V$ and not necessarily a basis.

The dual $V^{\prime}$ of a finitely generated and projective module $V$ is also finitely generated and projective, moreover the dual of the dual is canonically identified with the original module $V$. We state these properties without proof since they can be found in standard textbooks, e.g. [22].

Proposition 6.16. Let $V$ be a finitely generated and projective $\mathscr{M}_{A}$-module with a pair of dual bases $\left\{v_{i}, v_{i}^{\prime}: i=1, \ldots, n\right\}$. For any $v \in V$ let $v^{\prime \prime} \in V^{\prime \prime}:=$ ${ }_{A} \operatorname{Hom}\left(V^{\prime}, A\right)$ be defined by $v^{\prime \prime}\left(v^{\prime}\right):=v^{\prime}(v)$, for all $v^{\prime} \in V^{\prime}$. We have 
1) $\left\{v_{i}^{\prime}, v_{i}^{\prime \prime}: i=1, \ldots, n\right\}$ is a pair of dual bases for $V^{\prime}$

2) $V^{\prime}$ is a finitely generated and projective $A \mathscr{M}$-module

3) The canonical map $V \rightarrow V^{\prime \prime}, v \mapsto v^{\prime \prime}$ is an isomorphism of $\mathscr{M}_{A}$-modules

We now show that for finitely generated and projective $\mathscr{M}_{A}$-modules $V$ there is an isomorphism $\operatorname{Hom}_{A}(V, \Omega) \simeq \Omega \otimes_{A} V^{\prime}$. It is this property that allows us to construct connections on $V^{\prime}$ by a canonical dualization procedure.

Proposition 6.17. Let $V$ be a finitely generated and projective $\mathscr{M}_{A}$-module and let $\Omega$ be any ${ }_{A} \mathscr{M}_{A}$-module. Then there exists an ${ }_{A} \mathscr{M}$-module isomorphism (evaluation map)

$$
\iota: \Omega \otimes_{A} V^{\prime} \rightarrow \operatorname{Hom}_{A}(V, \Omega)
$$

defined by, for all $v \in V, \omega \in \Omega$ and $v^{\prime} \in V^{\prime},\left(\iota\left(\omega \otimes_{A} v^{\prime}\right)\right)(v):=\omega \cdot v^{\prime}(v)$.

If in addition $V$ is an ${ }^{H} \mathscr{M}_{A}$-module and $\Omega$ is an ${ }^{H}{ }_{A} \mathscr{M}_{A}$-module, then $\iota$ is an ${ }_{A}{ }_{A} \mathscr{M}$-module isomorphism.

Proof. The map $\iota$ is an ${ }_{A} \mathscr{M}$-module homomorphism, for all $a \in A, \omega \in \Omega$, $v^{\prime} \in V^{\prime}$ and $v \in V$,

$$
\begin{aligned}
\left(\iota\left(a \cdot \omega \otimes_{A} v^{\prime}\right)\right)(v) & =a \cdot \omega \cdot v^{\prime}(v)=a \cdot\left(\iota\left(\omega \otimes_{A} v^{\prime}\right)\right)(v) \\
& =\left(a \cdot \iota\left(\omega \otimes_{A} v^{\prime}\right)\right)(v) .
\end{aligned}
$$

Using a pair of dual bases $\left\{v_{i}, v_{i}^{\prime}: i=1, \ldots n\right\}$ for $V$, we can invert the map $\iota$ via

$$
\iota^{-1}: \operatorname{Hom}_{A}(V, \Omega) \rightarrow \Omega \otimes_{A} V^{\prime}, \quad P \mapsto \iota^{-1}(P)=\sum_{i=1}^{n} P\left(v_{i}\right) \otimes_{A} v_{i}^{\prime} .
$$

Let now $V$ be an ${ }^{H} \mathscr{M}_{A}$-module and $\Omega$ be an ${ }^{H}{ }_{A} \mathscr{M}_{A}$-module, then $V^{\prime}, \Omega$ $\otimes_{A} V^{\prime}, \operatorname{Hom}_{A}(V, \Omega)$ are ${ }_{A}{ }_{A} \mathscr{M}$-modules. The isomorphism $\iota$ respects the ${ }^{H} \mathscr{M}$ module structure, since for all $\xi \in H, \omega \in \Omega, v^{\prime} \in V^{\prime}$ and $v \in V$,

$$
\begin{aligned}
\left(\iota\left(\xi \triangleright\left(\omega \otimes_{A} v^{\prime}\right)\right)\right)(v) & =\left(\xi_{1} \triangleright \omega\right) \cdot\left(\xi_{2} \triangleright v^{\prime}\right)(v)=\left(\xi_{1} \triangleright \omega\right) \cdot\left(\xi_{2} \triangleright v^{\prime}\left(S\left(\xi_{3}\right) \triangleright v\right)\right) \\
(6.56) & =\xi_{1} \triangleright\left(\omega \cdot v^{\prime}\left(S\left(\xi_{2}\right) \triangleright v\right)\right)=\left(\xi \triangleright\left(\iota\left(\omega \otimes_{A} v^{\prime}\right)\right)\right)(v) .
\end{aligned}
$$


In order to construct the dual connection $\nabla^{\prime} \in{ }_{A} \operatorname{Con}\left(V^{\prime}\right)$ of a connection $\nabla \in \operatorname{Con}_{A}(V)$ we first investigate a certain dualization of $\mathbb{K}$-linear maps and $A$-linear maps (like the difference of two connections on $V$ ). Let $A$ be an algebra and let $\left(\Omega^{\bullet}, \wedge, \mathrm{d}\right)$ be a differential calculus over $A$. Let further $V$ be an $\mathscr{M}_{A}$-module and consider the $\mathbb{K}$-module of $\mathbb{K}$-linear maps $H_{\mathbb{K}}\left(V, V \otimes_{A} \Omega\right)$. We can associate to every $P \in H_{\mathbb{K}}\left(V, V \otimes_{A} \Omega\right)$ a $P^{H o m} \in{ }_{A} \operatorname{Hom}\left(V^{\prime}, \operatorname{Hom}_{\mathbb{K}}(V, \Omega)\right)$ by defining, for all $v^{\prime} \in V^{\prime}$ and $v \in V$,

$$
P^{H o m}\left(v^{\prime}\right)(v):=-\left(v^{\prime} \otimes \mathrm{id}\right) P(v)
$$

i.e.,

$$
P^{H o m}\left(v^{\prime}\right):=-\wedge \circ\left(v^{\prime} \otimes \mathrm{id}\right) \circ P
$$

In (6.57) $v^{\prime} \otimes$ id : $V \otimes_{A} \Omega \rightarrow A \otimes_{A} \Omega$ denotes the right $A$-linear map defined by, for all $v \in V$ and $\omega \in \Omega,\left(v^{\prime} \otimes\right.$ id $)\left(v \otimes_{A} \omega\right)=v^{\prime}(v) \otimes_{A} \omega$ and $\wedge: A \otimes_{A}$ $\Omega \rightarrow \Omega$ is the $A_{A} \mathscr{M}_{A}$-module isomorphism induced from the product in $\Omega^{\bullet}$, i.e. $\wedge\left(a \otimes_{A} \omega\right)=a \wedge \omega=a \cdot \omega$. The map $P^{H o m}$ is left $A$-linear, for all $a \in A$ and $v^{\prime} \in V^{\prime}$,

$$
\begin{aligned}
P^{H o m}\left(a \cdot v^{\prime}\right) & =-\wedge \circ\left(a \cdot v^{\prime} \otimes \mathrm{id}\right) \circ P=-\wedge \circ l_{a} \circ\left(v^{\prime} \otimes \mathrm{id}\right) \circ P \\
& =-l_{a} \circ \wedge \circ\left(v^{\prime} \otimes \mathrm{id}\right) \circ P=a \cdot P^{H o m}\left(v^{\prime}\right) .
\end{aligned}
$$

The association of $P^{H o m}$ to $P$ can be regarded as a $\mathbb{K}$-linear map

$$
{ }^{H o m}: \operatorname{Hom}_{\mathbb{K}}\left(V, V \otimes_{A} \Omega\right) \rightarrow\left({ }_{A} \operatorname{Hom}\left(V^{\prime}, \operatorname{Hom}_{\mathbb{K}}(V, \Omega)\right)\right)^{o p}, P \mapsto P^{H o m} .
$$

Furthermore, if $P \in \operatorname{Hom}_{A}\left(V, V \otimes_{A} \Omega\right)$ then $P^{H o m} \in\left({ }_{A} \operatorname{Hom}\left(V^{\prime}, \operatorname{Hom}_{A}(V\right.\right.$, $\Omega))^{o p}$, since for all $v^{\prime} \in V^{\prime}$, all maps in (6.57b) are right $A$-linear. Thus, (6.59) restricts to a $\mathbb{K}$-linear map

$$
{ }^{H o m}: \operatorname{Hom}_{A}\left(V, V \otimes_{A} \Omega\right) \rightarrow\left({ }_{A} \operatorname{Hom}\left(V^{\prime}, \operatorname{Hom}_{A}(V, \Omega)\right)\right)^{o p}
$$

We now explain the notation ( $)^{o p}$ used in these two last equations. Later we are going to consider an additional ${ }^{H} \mathscr{M}$-module structure on $V$ and $\Omega$, and hence also on $V^{\prime}, \operatorname{Hom}_{\mathbb{K}}(V, \Omega)$ and ${ }_{A} \operatorname{Hom}\left(V^{\prime}, H_{\mathbb{K}}(V, \Omega)\right)$. There are two different induced ${ }^{H} \mathscr{M}$-module structures on a $\mathbb{K}$-module of $\mathbb{K}$-linear maps between two ${ }^{H} \mathscr{M}$-modules, they are given by the adjoint actions and ${ }^{c o p}$. We denote by $\left({ }_{A} \operatorname{Hom}\left(V^{\prime}, \operatorname{Hom}_{\mathbb{K}}(V, \Omega)\right)\right)^{o p}$ the ${ }^{H} \mathscr{M}$-module with the ${ }^{c o p}$ adjoint action, which is the relevant one for left $A$-linear maps, cf. Proposition 4.4 . 
In order to avoid confusion when we later enrich the theory with ${ }^{H} \mathscr{M}$ module structures, we already write in this part $\left({ }_{A} \operatorname{Hom}\left(V^{\prime}, \operatorname{Hom}_{\mathbb{K}}(V, \Omega)\right)\right)^{o p}$ rather than ${ }_{A} \operatorname{Hom}\left(V^{\prime}, \operatorname{Hom}_{\mathbb{K}}(V, \Omega)\right)$, even if as $\mathbb{K}$-modules they coincide.

Notice that for a finitely generated and projective $\mathscr{M}_{A}$-module $V$ we can use the $A \mathscr{M}$-module isomorphism $\iota^{-1}$ of Proposition 6.17 to define the $\mathbb{K}$-linear map

$$
\text { ': } \operatorname{Hom}_{A}\left(V, V \otimes_{A} \Omega\right) \rightarrow\left({ }_{A} \operatorname{Hom}\left(V^{\prime}, \Omega \otimes_{A} V^{\prime}\right)\right)^{o p}, P \mapsto P^{\prime}=\iota^{-1} \circ P^{\text {Hom }} .
$$

We denoted this map by a prime because, as we will see later, it maps the difference of two connections on $V$ in the difference of their dual connections on $V^{\prime}$.

Let now $\nabla \in \operatorname{Con}_{A}(V) \subset \operatorname{Hom}_{\mathbb{K}}\left(V, V \otimes_{A} \Omega\right)$, then $\nabla^{\text {Hom }}$ is by construction a left $A$-linear map and thus can not be a candidate for a connection on the dual module $V^{\prime}$. This is why we associate to $\nabla \in \operatorname{Con}_{A}(V)$ the $\mathbb{K}$-linear map

$$
\widehat{\nabla}: V^{\prime} \rightarrow \operatorname{Hom}_{A}(V, \Omega), v^{\prime} \mapsto \widehat{\nabla}\left(v^{\prime}\right)=\mathrm{d} \circ v^{\prime}+\nabla^{H o m}\left(v^{\prime}\right) .
$$

We observe that, for all $v^{\prime} \in V^{\prime}$, we have the right $A$-linearity property of $\widehat{\nabla}\left(v^{\prime}\right)$, for all $a \in A$ and $v \in V$,

$$
\begin{aligned}
\widehat{\nabla}\left(v^{\prime}\right)(v \cdot a) & =\mathrm{d}\left(v^{\prime}(v \cdot a)\right)-\left(v^{\prime} \otimes \mathrm{id}\right) \nabla(v \cdot a) \\
& =\mathrm{d}\left(v^{\prime}(v)\right) \cdot a+v^{\prime}(v) \cdot \mathrm{d} a-\left(v^{\prime} \otimes \mathrm{id}\right) \nabla(v) \cdot a-\left(v^{\prime} \otimes \mathrm{id}\right)\left(v \otimes_{A} \mathrm{~d} a\right) \\
(6.63) & =\left(\widehat{\nabla}\left(v^{\prime}\right)(v)\right) \cdot a .
\end{aligned}
$$

Hence we have constructed a map

$$
\text { ^ }: \operatorname{Con}_{A}(V) \rightarrow\left(\operatorname{Hom}_{\mathbb{K}}\left(V^{\prime}, \operatorname{Hom}_{A}(V, \Omega)\right)\right)^{o p}, \nabla \mapsto \widehat{\nabla} .
$$

With the help of the ${ }_{A} \mathscr{M}$-module isomorphism $\iota^{-1}$ of Proposition 6.17 we also define the map (with slight abuse of notation we denote it with the same prime symbol used on homomorphisms in (6.61))

$$
\text { ': } \operatorname{Con}_{A}(V) \rightarrow\left(\operatorname{Hom}_{\mathbb{K}}\left(V^{\prime}, \Omega \otimes_{A} V^{\prime}\right)\right)^{o p}, \nabla \mapsto \nabla^{\prime}=\iota^{-1} \circ \widehat{\nabla} .
$$

Using (6.55), the map $\nabla^{\prime}$ acting on an arbitrary $v^{\prime} \in V^{\prime}$ explicitly reads

$$
\nabla^{\prime}\left(v^{\prime}\right)=\iota^{-1}\left(\widehat{\nabla}\left(v^{\prime}\right)\right)=\sum_{i=1}^{n}\left(\mathrm{~d}\left(v^{\prime}\left(v_{i}\right)\right)-\left(v^{\prime} \otimes \mathrm{id}\right) \nabla\left(v_{i}\right)\right) \otimes_{A} v_{i}^{\prime}
$$


We now show that $\nabla^{\prime}$ is a connection on $V^{\prime}$.

Theorem 6.18. Let $V$ be a finitely generated and projective $\mathscr{M}_{A}$-module and let $\left(\Omega^{\bullet}, \wedge, \mathrm{d}\right)$ be a differential calculus over $A$. The ' maps in (6.65) and in (6.61) provide an affine space isomorphism

$$
\text { ' }: \operatorname{Con}_{A}(V) \rightarrow{ }_{A} \operatorname{Con}\left(V^{\prime}\right), \nabla \mapsto \nabla^{\prime},
$$

where $\operatorname{Con}_{A}(V)$ and ${ }_{A} \operatorname{Con}\left(V^{\prime}\right)$ are affine spaces over the isomorphic $\mathbb{K}$ modules $\operatorname{Hom}_{A}\left(V, V \otimes_{A} \Omega\right)$ and $\left({ }_{A} \operatorname{Hom}\left(V^{\prime}, \Omega \otimes_{A} V^{\prime}\right)\right)^{o p}$, respectively.

Proof. We first have to show that, for all $\nabla \in \operatorname{Con}_{A}(V), \nabla^{\prime}$ defined by (6.65) is a connection on the $A \mathscr{M}$-module $V^{\prime}$. Using (6.66) and left $A$-linearity of $\nabla^{\text {Hom }}$ we have, for all $a \in A$ and $v^{\prime} \in V^{\prime}$,

$$
\begin{aligned}
\nabla^{\prime}\left(a \cdot v^{\prime}\right) & =\sum_{i=1}^{n}\left(\mathrm{~d}\left(a v^{\prime}\left(v_{i}\right)\right)-a \cdot\left(\left(v^{\prime} \otimes \mathrm{id}\right) \nabla\left(v_{i}\right)\right)\right) \otimes_{A} v_{i}^{\prime} \\
& =\sum_{i=1}^{n}\left(\mathrm{~d} a \cdot v^{\prime}\left(v_{i}\right)+a \cdot \mathrm{d}\left(v^{\prime}\left(v_{i}\right)\right)-a \cdot\left(\left(v^{\prime} \otimes_{\mathrm{id}}\right) \nabla\left(v_{i}\right)\right)\right) \otimes_{A} v_{i}^{\prime} \\
& =\mathrm{d} a \otimes_{A} \sum_{i=1}^{n} v_{i}^{\prime \prime}\left(v^{\prime}\right) \cdot v_{i}^{\prime}+a \cdot \nabla^{\prime}\left(v^{\prime}\right) \\
& =\mathrm{d} a \otimes_{A} v^{\prime}+a \cdot \nabla^{\prime}\left(v^{\prime}\right),
\end{aligned}
$$

where in the last two steps we have used also Proposition 6.16.

Next we show that the ' maps in (6.67) and (6.61) are an affine space map, for all $\nabla \in \operatorname{Con}_{A}(V), P \in \operatorname{Hom}_{A}\left(V, V \otimes_{A} \Omega\right)$ and $v^{\prime} \in V^{\prime}$,

$$
(\nabla+P)^{\prime}\left(v^{\prime}\right)=\iota^{-1}\left(\mathrm{~d} \circ v^{\prime}+(\nabla+P)^{H o m}\left(v^{\prime}\right)\right)
$$

$$
=\iota^{-1}\left(\mathrm{~d} \circ v^{\prime}+\nabla^{H o m}\left(v^{\prime}\right)+P^{H o m}\left(v^{\prime}\right)\right)=\left(\nabla^{\prime}+P^{\prime}\right)\left(v^{\prime}\right)
$$

where we have used that $H o m$ is $\mathbb{K}$-linear.

Finally, the ' maps in (6.67) and (6.61) are isomorphisms because the map Hom in (6.59) is invertible when the $\mathscr{M}_{A}$-module $V$ is finitely generated and projective. Explicitly, for all $T \in\left({ }_{A} \operatorname{Hom}\left(V^{\prime}, \operatorname{Hom}_{\mathbb{K}}(V, \Omega)\right)\right)^{o p}$ and $v \in V$, $T^{\operatorname{Hom}^{-1}}(v)=-\sum_{i=1}^{n} v_{i} \otimes_{A} T\left(v_{i}^{\prime}\right)(v)$, where $\left\{v_{i}, v_{i}^{\prime}: i=1, \ldots, n\right\}$ is a pair of dual bases for $V$.

Deformation of dual connections. The above detailed preliminary discussion on dual connections is helpful in order to understand their twist 
deformation. In this case we have a Hopf algebra $H$ that acts on all the modules encountered in the beginning of this subsection. We study these actions, in particular the maps Hom , $\iota$, and their composition ', are $H$-equivariant. We then twist deform the modules and consider the corresponding maps $\mathrm{Hom}_{\star}, \iota_{\star}$, and their composition ${ }^{{ }^{\star}}$. Their properties imply that deformation and dualization are compatible operations: given a connection $\nabla$, the dual of the twist deformed connection $\widetilde{D}_{\mathcal{F}}(\nabla)$ is equal to the twist deformation of the dual connection $\nabla^{\prime}$.

Let $H$ be a Hopf algebra, $A$ be an ${ }^{H} \mathscr{A}$-algebra, $V$ an ${ }^{H} \mathscr{M}_{A}$-module and $\left(\Omega^{\bullet}, \wedge, \mathrm{d}\right)$ a left $H$-covariant differential calculus over $A$. We recall that in this case $V \otimes_{A} \Omega$ is an ${ }^{H} \mathscr{M}_{A^{-}}$module and $V^{\prime}, \Omega \otimes_{A} V^{\prime}, H_{\mathbb{K}}(V, \Omega)$ are $H_{A} \mathscr{M}$-modules. Also the modules appearing in (6.59) are ${ }^{H} \mathscr{M}$-modules, in detail: The $H$-action on $H_{\mathbb{K}}\left(V, V \otimes_{A} \Omega\right)$ is given by the $H$-adjoint action - (obtained lifting the $H$-actions $\triangleright$ on $V$ and $V \otimes_{A} \Omega$ ). The $H$-action on $\left({ }_{A} \operatorname{Hom}\left(V^{\prime}, \operatorname{Hom}_{\mathbb{K}}(V, \Omega)\right)\right)^{o p}$ is the ${ }^{c o p}$ adjoint action, as required by the left $A$-linearity of these homomorphisms (cf. Proposition 4.4). It is obtained via a lift of the $H$-actions on $V^{\prime}$ and $\operatorname{Hom}_{\mathbb{K}}(V, \Omega)$, these latter are themselves adjoint actions $\triangleright$, obtained lifting the $H$-actions $\triangleright$ on $V$ and $\Omega$.

Throughout this subsection we assume $V$ to be finitely generated and projective as an $\mathscr{M}_{A}$-module. As proven in the end of Theorem 6.18 , the $\mathbb{K}$-linear map Hom is then invertible.

Lemma 6.19. The $\mathbb{K}$-linear map Hom in (6.59) is an ${ }^{H} \mathscr{M}$-module isomorphism, i.e. for all $P \in \operatorname{Hom}_{\mathbb{K}}\left(V, V \otimes_{A} \Omega\right)$ and all $\xi \in H,(\xi \triangleright P)^{H o m}=$ $\xi{ }^{\text {cop }} \mathrm{P}^{\mathrm{Hom}}$.

Proof. Recalling definition (6.57b) we obtain, for all $v^{\prime} \in V^{\prime}$

$$
\begin{aligned}
\left(\xi \triangleright{ }^{\text {cop }} P^{\text {Hom }}\right)\left(v^{\prime}\right) & =\xi_{2} \triangleright\left(P^{\text {Hom }}\left(S^{-1}\left(\xi_{1}\right) \triangleright v^{\prime}\right)\right) \\
& =-\xi_{2} \triangleright\left(\wedge \circ\left(S^{-1}\left(\xi_{1}\right) \triangleright v^{\prime} \otimes \mathrm{id}\right) \circ P\right) \\
& =-\wedge \circ\left(\left(\xi_{2} S^{-1}\left(\xi_{1}\right)\right) \triangleright v^{\prime} \otimes \mathrm{id}\right) \circ \xi_{3} \triangleright P \\
& =-\wedge \circ\left(v^{\prime} \otimes \mathrm{id}\right) \circ \xi \triangleright P=(\xi \triangleright P)^{H o m}
\end{aligned}
$$

In line three we used that $\wedge$ is $H$-equivariant.

Corollary 6.20. Since $\iota$ is an ${ }_{A}{ }_{A} \mathscr{M}$-module isomorphism (cf. Proposition 6.17) it follows that the' map (see (6.61)) is also an ${ }^{H} \mathscr{M}$-module isomorphism. 
We now consider a twist $\mathcal{F} \in H \otimes H$ and deform the modules relevant in the study of dual connections. In particular, we have the ${ }_{A_{\star}}^{\mathcal{F}^{\mathcal{M}}} \mathscr{\mathscr { M }}$-module isomorphisms $D_{\mathcal{F}}: V^{\prime}{ }_{\star} \rightarrow V_{\star}{ }^{\prime}$ and $D_{\mathcal{F}}: \operatorname{Hom}_{\mathbb{K}}(V, \Omega)_{\star}, \rightarrow \operatorname{Hom}_{\mathbb{K}}\left(V_{\star}, \Omega_{\star}\right)$ that intertwine between the actions of $H^{\mathcal{F}}$ on $\operatorname{Hom}_{\mathbb{K}}(V, \Omega)_{\star}$ and $V^{\prime}$, and the $\boldsymbol{D}_{\mathcal{F}}$ actions of $H^{\mathcal{F}}$ on $V_{\star}{ }^{\prime}$ and $H_{0} m_{\mathbb{K}}\left(V_{\star}, \Omega_{\star}\right)$. These $H^{\mathcal{F}}$-actions lift (cf. Proposition 4.4) to a cop adjoint action of $H^{\mathcal{F}}$ on $\left(\operatorname{Hom}_{\mathbb{K}}\left(V^{\prime}{ }_{\star}, \operatorname{Hom}_{\mathbb{K}}(V\right.\right.$, $\left.\left.\Omega)_{\star}\right)\right)^{o p}$ and a $\boldsymbol{F}_{\mathcal{F}}{ }^{c o p}$ adjoint action of $H^{\mathcal{F}}$ on $\left(H_{o m}\left(V_{\star}{ }^{\prime}, H_{o m}\left(V_{\star}, \Omega_{\star}\right)\right)\right)^{o p}$.

The two ${ }^{H^{\mathcal{F}}} \mathscr{M}$-modules $\left(\operatorname{Hom}_{\mathbb{K}}\left(V^{\prime}{ }_{\star}, \operatorname{Hom}_{\mathbb{K}}(V, \Omega)_{\star}\right)\right)^{\text {op }}$ and $\left(\operatorname{Hom}_{\mathbb{K}}\left(V_{\star}{ }^{\prime}\right.\right.$, $\left.\left.\operatorname{Hom}_{\mathbb{K}}\left(V_{\star}, \Omega_{\star}\right)\right)\right)^{o p}$ are isomorphic:

Lemma 6.21. Let $H$ be a Hopf algebra with twist $\mathcal{F} \in H \otimes H, A$ be an ${ }^{H} \mathscr{A}$-algebra, $V$ be an ${ }^{H} \mathscr{M}_{A}$-module and $\Omega$ be an ${ }^{H}{ }_{A} \mathscr{M}_{A}$-module. The $\mathbb{K}$ linear map

$$
\begin{aligned}
\operatorname{Ad}_{D_{\mathcal{F}}}:\left(\operatorname{Hom}_{\mathbb{K}}\left(V^{\prime}{ }_{\star}, \operatorname{Hom}_{\mathbb{K}}(V, \Omega)_{\star}\right)\right)^{o p} & \longrightarrow\left(\operatorname{Hom}_{\mathbb{K}}\left(V_{\star}{ }^{\prime}, \operatorname{Hom}_{\mathbb{K}}\left(V_{\star}, \Omega_{\star}\right)\right)\right)^{o p}, \\
(6.71) & T \longmapsto \operatorname{Ad}_{D_{\mathcal{F}}}(T)=D_{\mathcal{F}} \circ T \circ D_{\mathcal{F}}^{-1},
\end{aligned}
$$

is an ${ }^{H^{\mathcal{F}}} \mathscr{M}$-module isomorphism.

Furthermore, it restricts to an ${ }^{H^{\mathcal{F}}} \mathscr{M}$-module isomorphism

$\operatorname{Ad}_{D_{\mathcal{F}}}:\left(A_{\star} \operatorname{Hom}\left(V_{\star}^{\prime}, \operatorname{Hom}_{\mathbb{K}}(V, \Omega)_{\star}\right)\right)^{o p} \rightarrow\left(A_{\star} \operatorname{Hom}\left(V_{\star}{ }^{\prime}, \operatorname{Hom}_{\mathbb{K}}\left(V_{\star}, \Omega_{\star}\right)\right)\right)^{o p}$.

Proof. For all $T \in\left(\operatorname{Hom}_{\mathbb{K}}\left(V^{\prime}{ }_{\star}, \operatorname{Hom}_{\mathbb{K}}(V, \Omega)_{\star}\right)\right)^{o p}$ and $\xi \in H$ we have

$$
\begin{aligned}
\xi \triangleright_{\mathcal{F}}{ }^{c o p}\left(\operatorname{Ad}_{D_{\mathcal{F}}}(T)\right) & =\xi_{2_{\mathcal{F}}} \triangleright_{\mathcal{F}} \circ \operatorname{Ad}_{D_{\mathcal{F}}}(T) \circ S^{\mathcal{F}-1}\left(\xi_{1_{\mathcal{F}}}\right) \triangleright_{\mathcal{F}} \\
& =\xi_{2_{\mathcal{F}}} \triangleright_{\mathcal{F}} \circ D_{\mathcal{F}} \circ T \circ D_{\mathcal{F}}^{-1} \circ S^{\mathcal{F}-1}\left(\xi_{1_{\mathcal{F}}}\right) \triangleright_{\mathcal{F}} \\
& =D_{\mathcal{F}} \circ \xi_{2_{\mathcal{F}}} \circ T \circ S^{\mathcal{F}-1}\left(\xi_{1_{\mathcal{F}}}\right) \triangleright \circ D_{\mathcal{F}}^{-1} \\
& =\operatorname{Ad}_{D_{\mathcal{F}}}\left(\xi \triangleright{ }^{c o p} T\right),
\end{aligned}
$$

where we used that $D_{\mathcal{F}}$ intertwines between the $\boldsymbol{\nabla}$ and $\boldsymbol{}_{\mathcal{F}}$ adjoint actions, $D_{\mathcal{F}} \circ \xi \triangleright=\xi \triangleright_{\mathcal{F}} \circ D_{\mathcal{F}}$ (cf. Theorem 3.10). The map $\operatorname{Ad}_{D_{\mathcal{F}}}$ is obviously invertible.

Let now $T \in\left(A_{\star} \operatorname{Hom}\left(V^{\prime}{ }_{\star}, \operatorname{Hom}_{\mathbb{K}}(V, \Omega)_{\star}\right)\right)^{o p}$, i.e. for all $a \in A_{\star}$ and $v^{\prime} \in$ $V_{\star}^{\prime}, T\left(a \star v^{\prime}\right)=a \star T\left(v^{\prime}\right)$. Then, for all $v_{\star}^{\prime} \in V_{\star}{ }^{\prime}$,

$$
\begin{aligned}
\left(\operatorname{Ad}_{D_{\mathcal{F}}}(T)\right)\left(a \cdot v_{\star}^{\prime}\right) & =D_{\mathcal{F}}\left(T\left(D_{\mathcal{F}}^{-1}\left(a \cdot v_{\star}^{\prime}\right)\right)\right)=D_{\mathcal{F}}\left(T\left(a \star D_{\mathcal{F}}^{-1}\left(v_{\star}^{\prime}\right)\right)\right) \\
& =D_{\mathcal{F}}\left(a \star\left(T\left(D_{\mathcal{F}}^{-1}\left(v_{\star}^{\prime}\right)\right)\right)\right)=a \cdot D_{\mathcal{F}}\left(T\left(D_{\mathcal{F}}^{-1}\left(v_{\star}^{\prime}\right)\right)\right) \\
& =a \cdot\left(\left(\operatorname{Ad}_{D_{\mathcal{F}}}(T)\right)\left(v_{\star}^{\prime}\right)\right),
\end{aligned}
$$


where in the second and fourth equality we used that $D_{\mathcal{F}}$ is an ${ }^{H^{\mathcal{F}}}{ }_{A^{*}} \mathscr{M}$ module isomorphism.

Remark 6.22. We later consider a slight variation of the map $\operatorname{Ad}_{D_{\mathcal{F}}}$, namely the $\mathbb{K}$-linear map (denoted with abuse of notation also by $\operatorname{Ad}_{D_{\mathcal{F}}}$ )

$$
\begin{aligned}
\operatorname{Ad}_{D_{\mathcal{F}}}:\left(\operatorname{Hom}_{\mathbb{K}}\left(V^{\prime}{ }_{\star}, \Omega_{\star} \otimes_{A_{\star}} V^{\prime}{ }_{\star}\right)\right)^{o p} & \rightarrow\left(\operatorname{Hom}_{\mathbb{K}}\left(V_{\star}{ }^{\prime}, \Omega_{\star} \otimes_{A_{\star}} V_{\star}{ }^{\prime}\right)\right)^{o p}, \\
(6.75) & T \mapsto \operatorname{Ad}_{D_{\mathcal{F}}}(T)=\left(\operatorname{id} \otimes_{\star} D_{\mathcal{F}}\right) \circ T \circ D_{\mathcal{F}}^{-1} .
\end{aligned}
$$

With a similar calculation as in Lemma 6.21 one shows that this map is an $H^{\mathcal{F}} \mathscr{M}$-module isomorphism and that it restricts to the ${ }^{H^{\mathcal{F}}} \mathscr{M}$-module isomorphism (denoted by the same symbol)

$$
\operatorname{Ad}_{D_{\mathcal{F}}}:\left({ }_{A_{\star}} \operatorname{Hom}\left(V_{\star}^{\prime}, \Omega_{\star} \otimes_{A_{\star}} V^{\prime}{ }_{\star}\right)\right)^{o p} \longrightarrow\left(A_{\star} \operatorname{Hom}\left(V_{\star}{ }^{\prime}, \Omega_{\star} \otimes_{A_{\star}} V_{\star}^{\prime}\right)\right)^{o p} .
$$

As a first step towards the twist deformation of dual connections we study the map $\mathrm{Hom}_{\star}$ between homomorphisms of deformed modules. Let $\mathcal{F} \in H \otimes H$ be a twist of the Hopf algebra $H$. For all $P_{\star} \in H m_{\mathbb{K}}\left(V_{\star}, V_{\star} \otimes_{A_{\star}}\right.$ $\left.\Omega_{\star}\right)$ we define the map $P_{\star}^{H o m_{\star}} \in\left(A_{\star} \operatorname{Hom}\left(V_{\star}{ }^{\prime}, \operatorname{Hom}_{\mathbb{K}}\left(V_{\star}, \Omega_{\star}\right)\right)\right)^{o p}$ as in $(6.57 \mathrm{~b})$ by, for all $v_{\star}^{\prime} \in V_{\star}^{\prime}$,

$$
P_{\star}^{H o m_{\star}}\left(v_{\star}^{\prime}\right):=-\Lambda_{\star} \circ\left(v_{\star}^{\prime} \otimes_{\star} \text { id }\right) \circ P_{\star} .
$$

As in Lemma 6.19 we have an ${ }^{H^{\mathcal{F}}} \mathscr{M}$-module isomorphism

$$
\operatorname{Hom}_{\star}: \operatorname{Hom}_{\mathbb{K}}\left(V_{\star}, V_{\star} \otimes_{A_{\star}} \Omega_{\star}\right) \longrightarrow\left({ }_{A_{\star}} \operatorname{Hom}\left(V_{\star}{ }^{\prime}, \operatorname{Hom}_{\mathbb{K}}\left(V_{\star}, \Omega_{\star}\right)\right)\right)^{o p} .
$$

We compare this map with the map Hom , that, due to its $H$-equivariance, can be considered as the ${ }^{H^{\mathcal{F}}} \mathscr{M}$-module isomorphism

$$
\text { Hom }: \operatorname{Hom}_{\mathbb{K}}\left(V, V \otimes_{A} \Omega\right)_{\star} \longrightarrow\left({ }_{A} \operatorname{Hom}\left(V^{\prime}, \operatorname{Hom}_{\mathbb{K}}(V, \Omega)\right)\right)_{\star}^{o p} .
$$


Proposition 6.23. Let $\mathcal{F} \in H \otimes H$ be a twist of the Hopf algebra $H$. Then the following diagram of ${ }^{H^{\mathcal{F}}} \mathscr{M}$-module isomorphisms commutes:

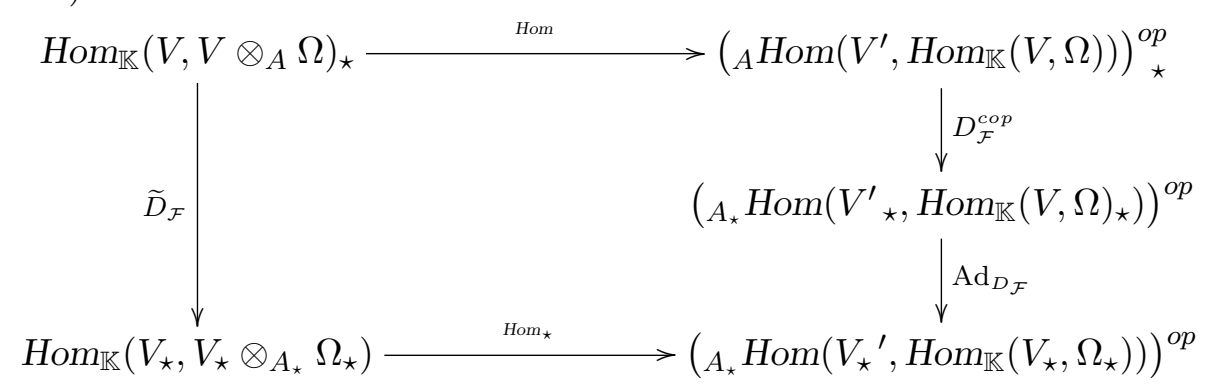

Proof. We consider any $P \in \operatorname{Hom}_{\mathbb{K}}\left(V, V \otimes_{A} \Omega\right)_{\star}$, follow the upper path in the diagram and act on an arbitrary element $v_{\star}^{\prime} \in V_{\star}{ }^{\prime}$,

$$
\begin{aligned}
\left(\operatorname{Ad}_{D_{\mathcal{F}}}\left(D_{\mathcal{F}}^{c o p}\left(P^{H o m}\right)\right)\right)\left(v_{\star}^{\prime}\right) & =D_{\mathcal{F}}\left(D_{\mathcal{F}}^{c o p}\left(P^{H o m}\right)\left(D_{\mathcal{F}}^{-1}\left(v_{\star}^{\prime}\right)\right)\right) \\
& =D_{\mathcal{F}}\left(\left(\overline{\mathrm{f}}_{\alpha}{ }^{c o p} P^{H o m}\right)\left(\overline{\mathrm{f}}^{\alpha} \rightarrow D_{\mathcal{F}}^{-1}\left(v_{\star}^{\prime}\right)\right)\right) \\
& =D_{\mathcal{F}}\left(\left(\overline{\mathrm{f}}_{\alpha}>P\right)^{H o m}\left(\overline{\mathrm{f}}^{\alpha} \rightarrow D_{\mathcal{F}}^{-1}\left(v_{\star}^{\prime}\right)\right)\right) \\
& =-D_{\mathcal{F}}\left(\wedge \circ\left(\left(\overline{\mathrm{f}}^{\alpha} \rightarrow D_{\mathcal{F}}^{-1}\left(v_{\star}^{\prime}\right)\right) \otimes \mathrm{id}\right) \circ\left(\overline{\mathrm{f}}_{\alpha} \triangleright P\right)\right) \\
& =-D_{\mathcal{F}}\left(\wedge \circ_{\star}\left(D_{\mathcal{F}}^{-1}\left(v_{\star}^{\prime}\right) \otimes \mathrm{id}\right) \circ_{\star} P\right) \\
& =-\wedge \circ D_{\mathcal{F}}\left(D_{\mathcal{F}}^{-1}\left(v_{\star}^{\prime}\right) \otimes \mathrm{id}\right) \circ D_{\mathcal{F}}(P) \\
& =-\wedge \circ \varphi \circ\left(v_{\star}^{\prime} \otimes_{\star} \mathrm{id}\right) \circ \varphi^{-1} \circ D_{\mathcal{F}}(P) \\
& =-\wedge_{\star} \circ\left(v_{\star}^{\prime} \otimes_{\star} \mathrm{id}\right) \circ \widetilde{D}_{\mathcal{F}}(P) . \\
(6.81) &
\end{aligned}
$$

In the third line we have used Lemma 6.19 , in the fourth line $(6.57 \mathrm{~b})$, in the fifth line that $\wedge$ is $H$-equivariant and in the sixth line this property and that $D_{\mathcal{F}}\left(Q \circ_{\star} \check{Q}\right)=D_{\mathcal{F}}(Q) \circ D_{\mathcal{F}}(\check{Q})$, which holds for arbitrary composable $\mathbb{K}$-linear maps $Q$ and $\check{Q}$. Then in line seven we used Theorem 5.21 and that id is $H$-equivariant. The last passage follows by noticing that $\wedge_{\star}=\wedge \circ \varphi$ and recalling the definition of $\widetilde{D}_{\mathcal{F}}(6.11)$.

We now follow the lower path in the diagram, from (6.77) we immediately obtain (6.81), and hence commutativity of the diagram. 
Remark 6.24. By restricting to right $A$-linear maps $\operatorname{Hom}_{A}\left(V, V \otimes_{A} \Omega\right)_{\star}$, we also obtain the following commutative diagram of $H^{\mathcal{F}} \mathscr{M}$-module isomorphisms:

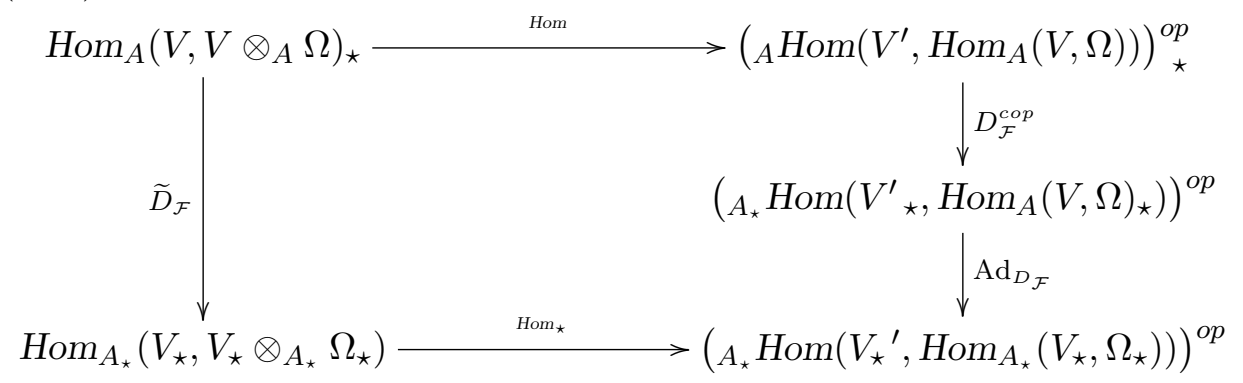

We now consider the map

$$
{ }^{\star}: \operatorname{Con}_{A_{\star}}\left(V_{\star}\right) \longrightarrow\left(\operatorname{Hom}_{\mathbb{K}}\left(V_{\star}{ }^{\prime}, \operatorname{Hom}_{A_{\star}}\left(V_{\star}, \Omega_{\star}\right)\right)\right)^{o p}
$$

defined by, for all $\nabla_{\star} \in \operatorname{Con}_{A_{\star}}\left(V_{\star}\right)$ and $v_{\star}^{\prime} \in V_{\star}^{\prime}$,

$$
\widehat{\nabla}_{\star}^{\star}\left(v_{\star}^{\prime}\right):=\mathrm{d} \circ v_{\star}^{\prime}+\nabla^{H o m_{\star}}\left(v_{\star}^{\prime}\right) .
$$

The corresponding map ${ }^{\wedge}$, defined in (6.64), can be seen as a map

$$
\text { }: \operatorname{Con}_{A}(V)_{\star} \longrightarrow\left(\operatorname{Hom}_{\mathbb{K}}\left(V^{\prime}, \operatorname{Hom}_{A}(V, \Omega)\right)\right)_{\star}^{o p},
$$

where we recall that $\operatorname{Con}_{A}(V)_{\star}$ differs from $\operatorname{Con}_{A}(V)$ just because it is seen as an affine space over the $H^{\mathcal{F}} \mathscr{M}$-module $\operatorname{Hom}_{A}\left(V, V \otimes_{A} \Omega\right)_{\star}$ rather than over the ${ }^{H} \mathscr{M}$-module $\operatorname{Hom}_{A}\left(V, V \otimes_{A} \Omega\right)$.

It is easy to see that the ${ }^{-}$and ${ }^{\wedge \star}$ maps close a commutative diagram, where the vertical arrows are given by deformation maps $D_{\mathcal{F}}, \widetilde{D}_{\mathcal{F}}$ and $D_{\mathcal{F}}^{\text {cop }}$. 
Corollary 6.25. Let $\mathcal{F} \in H \otimes H$ be a twist of the Hopf algebra $H$. Then the following diagram commutes:

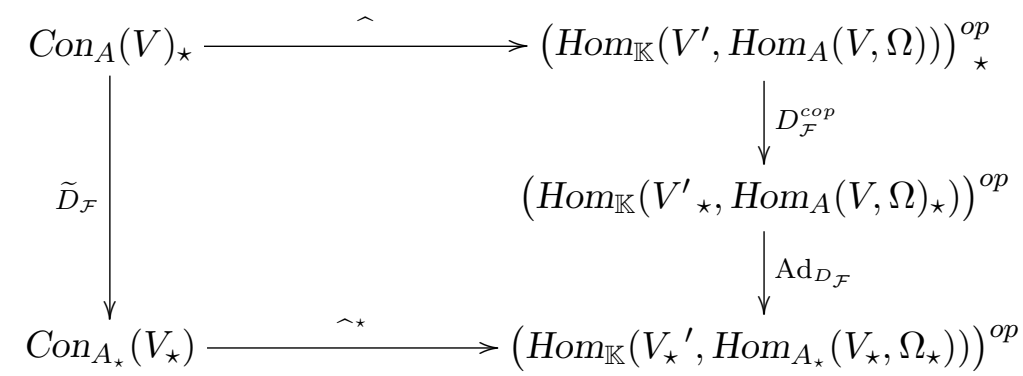

Proof. From Proposition 6.23 we know that this diagram holds for the second addend in the ${ }^{-}$and ${ }^{\star}$ maps in (6.64) and (6.84). Since the vertical arrows $D_{\mathcal{F}}^{\text {cop }}$ and $\operatorname{Ad}_{D_{\mathcal{F}}}$ are $\mathbb{K}$-linear maps, it remains to check the first addend. Use of $H$-equivariance of the differential d then implies, for all $v_{\star}^{\prime} \in V_{\star}{ }^{\prime}$,

$\left(\operatorname{Ad}_{D_{\mathcal{F}}}\left(D_{\mathcal{F}}^{c o p}\left(\widehat{\nabla}-\nabla^{H o m}\right)\right)\right)\left(v_{\star}^{\prime}\right)=\mathrm{d} \circ v_{\star}^{\prime}=\left({\widetilde{D_{\mathcal{F}}(\nabla}}^{\star}-\left(\widetilde{D}_{\mathcal{F}}(\nabla)\right)^{H o m_{\star}}\right)\left(v_{\star}^{\prime}\right)$.

The second step in the study of the deformation of the dual connection $\nabla^{\prime}=\iota^{-1} \circ \widehat{\nabla}$ of Theorem 6.18 is the study of the relation between the $\iota$ map of Proposition 6.17 and the corresponding one $\iota_{\star}$ between deformed modules. It is here that finitely generated and projective modules are needed. We denote by

$$
\iota_{\star}: \Omega_{\star} \otimes_{A_{\star}} V_{\star}^{\prime} \rightarrow \operatorname{Hom}_{A_{\star}}\left(V_{\star}, \Omega_{\star}\right)
$$

the ${ }^{H^{\mathcal{F}}} A_{\star} \mathscr{M}$-module isomorphism defined by, for all $v \in V_{\star}, \omega \in \Omega_{\star}, v_{\star}^{\prime} \in$ $V_{\star}{ }^{\prime},\left(\iota_{\star}\left(\omega \otimes_{A_{\star}} v_{\star}^{\prime}\right)\right)(v):=\omega \star v_{\star}^{\prime}(v)$. The maps $\iota$ and $\iota_{\star}$ close a commutative diagram where the vertical arrows are given by deformation maps $D_{\mathcal{F}}$ and $\varphi$. 
Lemma 6.26. Let $\mathcal{F} \in H \otimes H$ be a twist of the Hopf algebra $H$. Then the following diagram of ${ }_{A_{\star}}^{H^{\mathcal{M}}} \mathscr{M}$-module isomorphisms commutes:

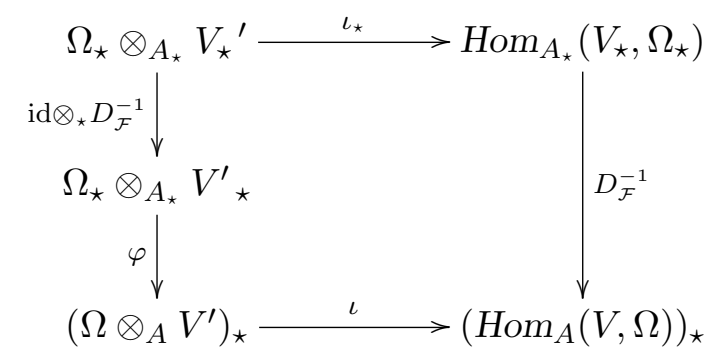

Proof. Using the explicit expression for $D_{\mathcal{F}}^{-1}$ (see Remark 3.11), we find, when following the upper path, for all $v \in V_{\star}, \omega \in \Omega_{\star}$ and $v_{\star}^{\prime} \in V_{\star}{ }^{\prime}$,

$$
\begin{aligned}
\left(D_{\mathcal{F}}^{-1}\left(\iota_{\star}\left(\omega \otimes_{A_{\star}} v_{\star}^{\prime}\right)\right)\right)(v) & =\overline{\mathrm{f}}^{\alpha} \triangleright\left(\omega \star v_{\star}^{\prime}\left(\chi S\left(\overline{\mathrm{f}}_{\alpha}\right) \triangleright v\right)\right) \\
& =\left(\overline{\mathrm{f}}_{1}^{\alpha} \overline{\mathrm{f}}^{\beta} \triangleright \omega\right) \cdot \overline{\mathrm{f}}_{2}^{\alpha} \overline{\mathrm{f}}_{\beta} \triangleright\left(v_{\star}^{\prime}\left(\chi S\left(\overline{\mathrm{f}}_{\alpha}\right) \triangleright v\right)\right) \\
& =\left(\overline{\mathrm{f}}^{\alpha} \triangleright \omega\right) \cdot \overline{\mathrm{f}}_{\alpha_{1}} \overline{\mathrm{f}}^{\beta} \triangleright\left(v_{\star}^{\prime}\left(\chi S\left(\overline{\mathrm{f}}_{\beta}\right) S\left(\overline{\mathrm{f}}_{\alpha_{2}}\right) \triangleright v\right)\right) \\
& =\left(\overline{\mathrm{f}}^{\alpha} \triangleright \omega\right) \cdot\left(\overline{\mathrm{f}}_{\alpha} \triangleright D_{\mathcal{F}}^{-1}\left(v_{\star}^{\prime}\right)\right)(v),
\end{aligned}
$$

where in line three we have used the twist cocycle property (3.1a). Following the lower path we find the same expression, for all $v \in V_{\star}, \omega \in \Omega_{\star}$ and $v_{\star}^{\prime}$ $\in V_{\star}{ }^{\prime}$,

$$
\begin{aligned}
\iota\left(\varphi\left(\omega \otimes_{A_{\star}} D_{\mathcal{F}}^{-1}\left(v_{\star}^{\prime}\right)\right)\right)(v) & =\iota\left(\left(\overline{\mathrm{f}}^{\alpha} \triangleright \omega\right) \otimes_{A}\left(\overline{\mathrm{f}}_{\alpha} \triangleright D_{\mathcal{F}}^{-1}\left(v_{\star}^{\prime}\right)\right)\right)(v) \\
& =\left(\overline{\mathrm{f}}^{\alpha} \triangleright \omega\right) \cdot\left(\overline{\mathrm{f}}_{\alpha} \triangleright D_{\mathcal{F}}^{-1}\left(v_{\star}^{\prime}\right)\right)(v) .
\end{aligned}
$$

It is convenient to consider the following ${ }^{H} \mathscr{M}$-module isomorphism induced by the ${ }_{A}^{H} \mathscr{M}$-module isomorphism $\iota^{-1}$ (and denoted with a slight abuse of notation by the same symbol)

$$
\begin{aligned}
\iota^{-1}:\left(\operatorname{Hom}_{\mathbb{K}}\left(V^{\prime}, \operatorname{Hom}_{A}(V, \Omega)\right)\right)^{o p} & \rightarrow\left(\operatorname{Hom}_{\mathbb{K}}\left(V^{\prime}, \Omega \otimes_{A} V^{\prime}\right)\right)^{o p}, \\
T & \mapsto \iota^{-1} \circ T .
\end{aligned}
$$

With this definition the maps ' in (6.61) and (6.65) are just the compositions $'=\iota^{-1} \circ{ }^{H o m}$ and ${ }^{\prime}=\iota^{-1} \circ^{-}$. Since the map $\iota^{-1}$ is $H$-equivariant, it can also be seen as an isomorphism between $H^{\mathcal{F}} \mathscr{M}$-modules, $\iota^{-1}:\left(\operatorname{Hom}_{\mathbb{K}}\left(V^{\prime}\right.\right.$, $\left.\operatorname{Hom}_{A}(V, \Omega)\right)_{\star}^{o p} \rightarrow\left(\operatorname{Hom}_{\mathbb{K}}\left(V^{\prime}, \Omega \otimes_{A} V^{\prime}\right)\right)_{\star}^{o p}$. 
Similarly the isomorphism $\iota_{\star}$ in $(6.88)$ induces the ${ }^{H^{\mathcal{F}}} \mathscr{M}$-module isomorphism

$$
\iota_{\star}^{-1}:\left(\operatorname{Hom}_{\mathbb{K}}\left(V_{\star}{ }^{\prime}, \operatorname{Hom}_{A_{\star}}\left(V_{\star}, \Omega_{\star}\right)\right)\right)^{o p} \rightarrow\left(\operatorname{Hom}_{\mathbb{K}}\left(V_{\star}{ }^{\prime}, \Omega_{\star} \otimes_{A_{\star}} V_{\star}{ }^{\prime}\right)\right)^{o p},
$$

Proposition 6.27. Let $\mathcal{F} \in H \otimes H$ be a twist of the Hopf algebra $H$. Then the following diagram of ${ }^{H^{\mathcal{F}}} \mathscr{M}$-module isomorphisms commutes:

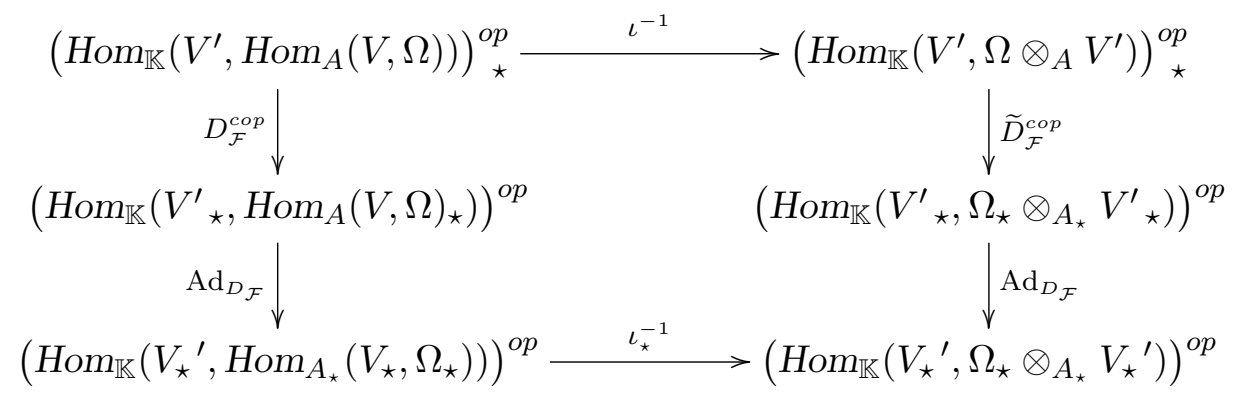

(where we recall the abuse of notation in the definition of $\operatorname{Ad}_{D_{\mathcal{F}}}$ in $R e$ mark 6.22).

Proof. We consider an arbitrary $T \in\left(\operatorname{Hom}_{\mathbb{K}}\left(V^{\prime}, H_{A} m_{A}(V, \Omega)\right)\right)_{\star}^{o p}$, follow the upper path in the diagram and act on an arbitrary $v_{\star}^{\prime} \in V_{\star}{ }^{\prime}$,

$$
\begin{aligned}
\operatorname{Ad}_{D_{\mathcal{F}}}\left(\widetilde{D}_{\mathcal{F}}^{c o p}\left(\iota^{-1}(T)\right)\right)\left(v_{\star}^{\prime}\right) & \\
& =\left(\mathrm{id} \otimes_{\star} D_{\mathcal{F}}\right)\left(\widetilde{D}_{\mathcal{F}}^{c o p}\left(\iota^{-1}(T)\right)\left(D_{\mathcal{F}}^{-1}\left(v_{\star}^{\prime}\right)\right)\right) \\
& =\left(\left(\mathrm{id} \otimes_{\star} D_{\mathcal{F}}\right) \circ \varphi^{-1}\right)\left(\left(\overline{\mathrm{f}}_{\alpha}{ }^{c o p} \iota^{-1}(T)\right)\left(\overline{\mathrm{f}}^{\alpha} \triangleright D_{\mathcal{F}}^{-1}\left(v_{\star}^{\prime}\right)\right)\right) \\
& =\left(\left(\mathrm{id} \otimes_{\star} D_{\mathcal{F}}\right) \circ \varphi^{-1} \circ \iota^{-1}\right)\left(\left(\overline{\mathrm{f}}_{\alpha} \triangleright^{c o p} T\right)\left(\overline{\mathrm{f}}^{\alpha} \rightarrow D_{\mathcal{F}}^{-1}\left(v_{\star}^{\prime}\right)\right)\right) \\
& =\left(\iota_{\star}^{-1} \circ D_{\mathcal{F}}\right)\left(D_{\mathcal{F}}^{c o p}(T)\left(D_{\mathcal{F}}^{-1}\left(v_{\star}^{\prime}\right)\right)\right) \\
& =\iota_{\star}^{-1}\left(\operatorname{Ad}_{D_{\mathcal{F}}}\left(D_{\mathcal{F}}^{c o p}(T)\right)\right)\left(v_{\star}^{\prime}\right),
\end{aligned}
$$

which is exactly the expression we obtain by following the lower path in the diagram. In line two we have used the definition of $\widetilde{D}_{\mathcal{F}}^{\text {cop }}$ (cf. Theorem 6.6), in line three that $\iota^{-1}$ is $H$-equivariant and then (6.92), finally in line four Lemma 6.26 in the form $\left(\mathrm{id} \otimes_{\star} D_{\mathcal{F}}\right) \circ \varphi^{-1} \circ \iota^{-1}=\iota_{\star}^{-1} \circ D_{\mathcal{F}}$. 
The ${ }^{\prime} \star$ maps between deformed modules corresponding to the prime maps in (6.65) and (6.61) (that with a slight abuse of notation are denoted with the same symbol) are defined by

$$
{ }^{\iota_{\star}}: \operatorname{Con}_{A_{\star}}\left(V_{\star}\right) \rightarrow{ }_{A_{\star}} \operatorname{Con}\left(V_{\star}{ }^{\prime}\right), \nabla_{\star} \mapsto \nabla_{\star}^{\prime \star}=\iota_{\star}^{-1} \circ \widehat{\nabla}_{\star}^{\star}
$$

and

$$
\begin{aligned}
\iota_{\star}: \operatorname{Hom}_{A_{\star}}\left(V_{\star}, V_{\star} \otimes_{A_{\star}} \Omega_{\star}\right) & \rightarrow\left({ }_{A_{\star}} \operatorname{Hom}\left(V_{\star}{ }^{\prime}, \Omega_{\star} \otimes_{A_{\star}} V_{\star}{ }^{\prime}\right)\right)^{o p}, \\
P_{\star} & \mapsto P_{\star}^{\prime \star}=\iota_{\star}^{-1} \circ P_{\star}^{H o m_{\star}},
\end{aligned}
$$

or, using the $\iota_{\star}^{-1}$ map in (6.93), simply by the compositions ${ }^{\prime \star}=\iota_{\star}^{-1} \circ{ }^{\mathrm{Hom}_{\star}}$ and ${ }^{\prime \star}=\iota_{\star}^{-1} \iota^{-\star}$.

Theorem 6.18 and Lemma 6.19 imply that these maps constitute an affine space isomorphism, where $\operatorname{Con}_{A_{\star}}\left(V_{\star}\right)$ and ${ }_{A_{\star}} \operatorname{Con}\left(V_{\star}{ }^{\prime}\right)$ are affine spaces over the ${ }^{H^{\mathcal{F}}} \mathscr{M}$-modules of right $A_{\star}$-linear maps $\operatorname{Hom}_{A_{\star}}\left(V_{\star}, V_{\star} \otimes_{A_{\star}} \Omega_{\star}\right)$ and of left $A_{\star}$-linear maps $\left({ }_{A_{\star}} \operatorname{Hom}\left(V_{\star}{ }^{\prime}, \Omega_{\star} \otimes_{A_{\star}} V_{\star}{ }^{\prime}\right)\right)^{o p}$, respectively.

We can now finally prove the main theorem of this subsection stating that deforming the dual connection is equivalent to dualizing the deformed connection.

Theorem 6.28. Let $H$ be a Hopf algebra with twist $\mathcal{F} \in H \otimes H, A$ be an ${ }^{H} \mathscr{A}$-algebra, $V$ be a finitely generated and projective ${ }^{H} \mathscr{M}_{A}$-module and $\left(\Omega^{\bullet}, \wedge, \mathrm{d}\right)$ be a left $H$-covariant differential calculus over $A$. Then the following diagram of isomorphisms between affine spaces of connections over $H^{\mathcal{F}} \mathscr{M}$-modules commutes:

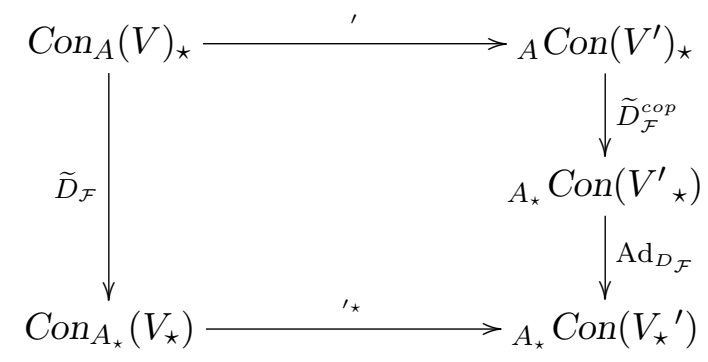

Proof. Combining the commutative diagrams of Corollary 6.25 and Proposition 6.27 we obtain commutativity of the above diagram regarded as maps. The commutativity of the diagram regarded as affine space maps follows by combining the diagrams of Remark 6.24 and Proposition 6.27. The vertical maps are obviously invertible, the horizontal ones are also isomorphisms (recall Theorem 6.18). 
Dual connections on ${ }^{\boldsymbol{H}} \mathscr{M}_{\boldsymbol{A}}$-modules. We consider the case where $V$ is an ${ }^{H} \mathscr{M}_{A}$-module, then all the modules encountered in this subsection are also ${ }_{A} \mathscr{M}_{A}$-modules, we list them: $V^{\prime}, \Omega \otimes_{A} V^{\prime}, \operatorname{Hom}_{\mathbb{K}}(V, \Omega), H_{A}(V, \Omega)$, $H_{\mathbb{K}}\left(V, V \otimes_{A} \Omega\right), H_{A}\left(V, V \otimes_{A} \Omega\right),\left(H_{\mathbb{K}}\left(V^{\prime}, \Omega \otimes_{A} V^{\prime}\right)\right)^{o p},\left({ }_{A} \operatorname{Hom}\left(V^{\prime}\right.\right.$, $\left.\left.\Omega \otimes_{A} V^{\prime}\right)\right)^{o p}$ as well as $\left(\operatorname{Hom}_{\mathbb{K}}\left(V^{\prime}, \operatorname{Hom}_{\mathbb{K}}(V, \Omega)\right)\right)^{\text {op }}$ and $\left({ }_{A} \operatorname{Hom}\left(V^{\prime}, \operatorname{Hom}_{\mathbb{K}}(V\right.\right.$, $\Omega))^{o p}$. For example, Proposition 4.4 implies that for all $T \in\left(\operatorname{Hom}_{\mathbb{K}}\left(V^{\prime}\right.\right.$, $\left.\left.\operatorname{Hom}_{\mathbb{K}}(V, \Omega)\right)\right)^{o p}$ and $a \in A$ the right $A$-module structure is given by $T . o p$ $a=\boldsymbol{r}_{a} \circ T$, where $\boldsymbol{r}: A \rightarrow{ }_{A} \operatorname{End}\left(\operatorname{Hom}_{\mathbb{K}}(V, \Omega)\right)$ and, for all $P \in \operatorname{Hom}_{\mathbb{K}}(V, \Omega)$, $\boldsymbol{r}_{a}(P)=P \circ l_{a}$. Similarly the left $A$-module structure is given by $a \cdot{ }^{o p} T=$ $T \circ \boldsymbol{r}_{a}$ where now $\boldsymbol{r}: A \rightarrow{ }_{A} \operatorname{End}\left(V^{\prime}\right)$.

It is now straightforward to prove that in this case the isomorphisms $\iota$ in (6.53), ${ }^{H o m}$ in (6.59) and (6.60), and hence ' in (6.61) are ${ }^{H}{ }_{A} \mathscr{M}_{A \text {-module }}$ isomorphisms. Thus we have

Corollary 6.29. Let $V$ be an ${ }^{H}{ }_{A} \mathscr{M}_{A}$-module. Then, the isomorphism ' : $\operatorname{Con}_{A}(V) \rightarrow{ }_{A} \operatorname{Con}\left(V^{\prime}\right)$ of Theorem 6.18 is an affine space isomorphism, where $\operatorname{Con}_{A}(V)$ and ${ }_{A} \operatorname{Con}\left(V^{\prime}\right)$ are affine spaces over the isomorphic ${ }^{H}{ }_{A^{\prime}} \mathscr{M}_{A^{-}}$ modules $\operatorname{Hom}_{A}\left(V, V \otimes_{A} \Omega\right)$ and $\left({ }_{A} \operatorname{Hom}\left(V^{\prime}, \Omega \otimes_{A} V^{\prime}\right)\right)^{o p}$, respectively.

Similarly, twist deformation of connections on ${ }^{H}{ }_{A} \mathscr{M}_{A}$-modules leads to Theorem 6.28, where now all the maps in the commutative diagram are isomorphisms between affine spaces of connections over ${ }^{H^{\mathcal{F}}}{ }_{A_{\star}} \mathscr{M}_{A_{\star}}$-modules. Indeed, all the maps in the commutative diagram of ${ }^{H^{\mathcal{F}}}{ }_{A_{\star}} \mathscr{M}_{A_{\star}}$-modules underlying the diagram in Theorem 6.28 are ${ }_{A_{\star}}^{H^{\mathcal{F}}} \mathscr{M}_{A_{\star}}$-module isomorphisms. (The map $\operatorname{Ad}_{D_{\mathcal{F}}}$ is an ${ }_{A_{\star}}^{H^{\mathcal{F}}} \mathscr{M}_{A_{\star}}$-module isomorphisms simply because all the other maps in this commutative diagram are ${ }_{A_{\star}}^{H^{\mathcal{F}}} \mathscr{M}_{A_{\star}}$-module isomorphisms).

\subsection{Connections on tensor products of modules and dual modules}

Combining the results of Corollary 6.29 on dual connections and of Theorem 6.13 on right to left connections we immediately obtain the following

Corollary 6.30. Let $(H, \mathcal{R})$ be a quasitriangular Hopf algebra, $A$ be a quasi-commutative ${ }^{H} \mathscr{A}$-algebra, $V$ be a strong quasi-commutative ${ }^{{ }^{H}} \mathscr{M}_{A^{-}}$ module and $\left(\Omega^{\bullet}, \wedge, \mathrm{d}\right)$ be a graded quasi-commutative left $H$-covariant differential calculus over $A$. Let further $V$ be finitely generated and projective as an $\mathscr{M}_{A}$-module and denote by $V^{\prime}=\operatorname{Hom}_{A}(V, A)$ the dual ${ }^{H}{ }_{A} \mathscr{M}_{A}$-module 
of $V$. Then there is an affine space isomorphism

$$
\operatorname{Con}_{A}(V) \stackrel{,}{\longrightarrow}{ }_{A} \operatorname{Con}\left(V^{\prime}\right) \stackrel{\widetilde{D}_{\mathcal{R}}^{-1}}{\longrightarrow} \operatorname{Con}_{A}\left(V^{\prime}\right)
$$

where the sets of right connections $\mathrm{Con}_{A}(V)$ and $\mathrm{Con}_{A}\left(V^{\prime}\right)$ are affine spaces over the isomorphic ${ }^{H} \mathscr{M}_{A}$-modules $\operatorname{Hom}_{A}\left(V, V \otimes_{A} \Omega\right)$ and $H_{A} m_{A}\left(V^{\prime}, V^{\prime} \otimes_{A}\right.$ $\Omega)$, respectively. Explicitly, given a right connection $\nabla \in \operatorname{Con}_{A}(V)$ the right connection on the dual module is $\widetilde{D}_{\mathcal{R}}^{-1}\left(\nabla^{\prime}\right) \in \operatorname{Con}_{A}\left(V^{\prime}\right)$.

This result allows to induce from a connection on $V$ a connection on the tensor algebra generated by $V$ and $V^{\prime}$. In other words, given a connection on vector fields (elements of $V$ ) we extend it to a connection on covariant and contravariant tensor fields (elements of the tensor algebra generated by $V$ and $\left.V^{\prime}\right)$.

Corollary 6.31. In the hypotheses of Corollary 6.30, given a right connection $\nabla \in \operatorname{Con}_{A}(V)$ we induce the right connection $\widetilde{D}_{\mathcal{R}}^{-1}\left(\nabla^{\prime}\right) \in \operatorname{Con}_{A}\left(V^{\prime}\right)$. Sums of the $\nabla$ and $\widetilde{D}_{\mathcal{R}}^{-1}\left(\nabla^{\prime}\right)$ connections as in Theorem 6.9 allow to extend the connection $\nabla$ to the tensor algebra generated by $V$ and $V^{\prime}$.

Proof. This construction is unambiguous due to the associativity of the sum of connections, which is proven in Theorem 6.10.

Remark 6.32. The deformation of the sum of a connection and its dual connection is canonical, in fact we have already shown that sum, dualization and the left to right isomorphism $\widetilde{D}_{\mathcal{R}}^{-1}$ are compatible with deformation, hence their composition is also compatible.

\section{Curvature}

After reviewing the basic definitions we investigate the behavior of the curvature of a connection under twist deformation. The twist deformed curvature in general differs from the curvature of the twist deformed connection, hence flat connections are twisted in non flat ones and vice versa. We then explicitly calculate the curvature of the sum of two connections, $\nabla_{V} \oplus_{\mathcal{R}} \nabla_{W}$, and find that it is in general not simply given by the sum of the individual curvatures. 


\subsection{Definitions}

A connection on an $\mathscr{M}_{A}$-module $V$ extends as usual to a $\mathbb{K}$-linear map from $V \otimes_{A} \Omega^{\bullet}$ to $V \otimes_{A} \Omega^{\bullet}$ (cf. (7.3)), where $\left(\Omega^{\bullet}, \wedge, \mathrm{d}\right)$ is a differential calculus over $A$. For later purposes we analyse in subsequent steps this construction. We then define the curvature of a connection.

Lemma 7.1. Let $V$ be an $\mathscr{M}_{A}$-module, $\left(\Omega^{\bullet}, \wedge, \mathrm{d}\right)$ a differential calculus over the algebra $A$, and $\nabla \in \operatorname{Con}_{A}(V)$. We define the $\mathbb{K}$-linear map $\nabla^{\bullet}$ : $V \otimes \Omega^{\bullet} \rightarrow V \otimes_{A} \Omega^{\bullet}$ by

$$
\nabla^{\bullet}:=(\mathrm{id} \otimes \wedge) \circ(\nabla \otimes \mathrm{id})+\pi \circ(\mathrm{id} \otimes \mathrm{d})
$$

Here $\pi: V \otimes \Omega^{\bullet} \rightarrow V \otimes_{A} \Omega^{\bullet}$ is the canonical projection and id $\otimes \wedge:\left(V \otimes_{A}\right.$ $\left.\Omega^{\bullet}\right) \otimes \Omega^{\bullet} \rightarrow V \otimes_{A} \Omega^{\bullet}$ is the right A-linear map defined by, for all $v \in V$ and $\omega, \tilde{\omega} \in \Omega^{\bullet}$,

$$
(\operatorname{id} \otimes \wedge)\left(\left(v \otimes_{A} \omega\right) \otimes \tilde{\omega}\right):=\left(v \otimes_{A} \omega\right) \wedge \tilde{\omega}:=v \otimes_{A} \omega \wedge \tilde{\omega}
$$

and extended to all $\left(V \otimes_{A} \Omega^{\bullet}\right) \otimes \Omega^{\bullet}$ by $\mathbb{K}$-linearity

The map $\nabla^{\bullet}$ induces a well-defined map (still denoted by $\nabla^{\bullet}$ for ease of notation) on the quotient, $\nabla^{\bullet}: V \otimes_{A} \Omega^{\bullet} \rightarrow V \otimes_{A} \Omega^{\bullet}$. Explicitly, for all $v \in V$ and $\omega \in \Omega^{\bullet}$,

$$
\nabla^{\bullet}\left(v \otimes_{A} \omega\right)=\nabla v \wedge \omega+v \otimes_{A} \mathrm{~d} \omega
$$

The map $\nabla^{\bullet}: V \otimes_{A} \Omega^{\bullet} \rightarrow V \otimes_{A} \Omega^{\bullet}$ satisfies, for all $v \in V, a \in A$ and $\omega \in \Omega^{\bullet}$ of homogeneous degree,

$$
\nabla^{\bullet}\left(\left(v \otimes_{A} \omega\right) a\right)=\left(\nabla^{\bullet}\left(v \otimes_{A} \omega\right)\right) a+(-1)^{\operatorname{deg}(\omega)} v \otimes_{A} \omega \wedge \mathrm{d} a
$$

Proof. The map (7.1) induces a well-defined map on the quotient, since it annihilates the submodule $\mathcal{N}_{V, \Omega} \bullet=\operatorname{ker}(\pi)$, i.e. $\nabla^{\bullet}\left(\mathcal{N}_{V, \Omega} \bullet\right)=\{0\}$. Indeed, for all $a \in A, v \in V$ and $\omega \in \Omega^{\bullet}$,

$$
\begin{aligned}
\nabla^{\bullet}(v \cdot a \otimes \omega) & =\nabla(v \cdot a) \wedge \omega+v \cdot a \otimes_{A} \mathrm{~d} \omega \\
& =(\nabla v) a \wedge \omega+v \otimes_{A} \mathrm{~d} a \wedge \omega+v \cdot a \otimes_{A} \mathrm{~d} \omega \\
& =\nabla v \wedge a \omega+v \otimes_{A}(\mathrm{~d} a \wedge \omega+a \mathrm{~d} \omega) \\
& =\nabla v \wedge a \omega+v \otimes_{A} \mathrm{~d}(a \omega) \\
& =\nabla^{\bullet}(v \otimes a \omega) .
\end{aligned}
$$


Property (7.4) hold because, for all $v \in V, a \in A$ and $\omega \in \Omega^{\bullet}$ of homogeneous degree,

$$
\begin{aligned}
\nabla^{\bullet}\left(v \otimes_{A} \omega a\right) & =\nabla v \wedge \omega a+v \otimes_{A} \mathrm{~d}(\omega a) \\
& =\nabla v \wedge \omega a+v \otimes_{A}\left((\mathrm{~d} \omega) a+(-1)^{\operatorname{deg}(\omega)} \omega \wedge \mathrm{d} a\right) \\
& =\left(\nabla^{\bullet}\left(v \otimes_{A} \omega\right)\right) a+(-1)^{\operatorname{deg}(\omega)} v \otimes_{A} \omega \wedge \mathrm{d} a .
\end{aligned}
$$

Definition 7.2. Let $\nabla \in \operatorname{Con}_{A}(V)$. The curvature of $\nabla$ is the $\mathbb{K}$-linear map defined by

$$
R_{\nabla}:=\nabla^{\bullet} \circ \nabla: V \rightarrow V \otimes_{A} \Omega^{2}
$$

The curvature is right $A$-linear, i.e. $R_{\nabla} \in \operatorname{Hom}_{A}\left(V, V \otimes_{A} \Omega^{2}\right)$, indeed, for all $a \in A$ and $v \in V$,

$$
\begin{aligned}
R_{\nabla}(v \cdot a) & =\nabla^{\bullet}(\nabla(v \cdot a))=\nabla^{\bullet}\left((\nabla v) a+v \otimes_{A} \mathrm{~d} a\right) \\
& =\nabla^{\bullet}(\nabla v) a-\nabla v \wedge \mathrm{d} a+\nabla v \wedge \mathrm{d} a+v \otimes_{A} \mathrm{~d} \mathrm{~d} a \\
& =\nabla^{\bullet}(\nabla v) a=R_{\nabla}(v) a .
\end{aligned}
$$

\subsection{Curvature of deformed connections}

Let $H$ be a Hopf algebra with twist $\mathcal{F} \in H \otimes H, A$ be an ${ }^{H} \mathscr{A}$-algebra, $V$ be an ${ }^{H} \mathscr{M}_{A}$-module and $\left(\Omega^{\bullet}, \wedge\right.$, d) be a left $H$-covariant differential calculus over $A$. We denote by $H^{\mathcal{F}}, A_{\star}, V_{\star}$ and $\left(\Omega^{\bullet}, \wedge_{\star}, \mathrm{d}\right)$ the deformations of $H, A, V$ and $\left(\Omega^{\bullet}, \wedge, \mathrm{d}\right)$ (obtained applying Theorems 3.2, 3.4, 3.5 and Proposition 6.4, respectively).

Consider an arbitrary connection $\nabla_{\star} \in \operatorname{Con}_{A_{\star}}\left(V_{\star}\right)$, then, by Lemma 7.1, we have a well-defined extension $\nabla_{\star}^{\bullet_{\star}}: V_{\star} \otimes_{A_{\star}} \Omega_{\star}^{\bullet} \rightarrow V_{\star} \otimes_{A_{\star}} \Omega_{\star}^{\bullet}$. The $\mathbb{K}$-linear $\operatorname{map} \nabla_{\star}^{\bullet_{\star}}: V_{\star} \otimes_{\star} \Omega_{\star}^{\bullet} \rightarrow V_{\star} \otimes_{A_{\star}} \Omega_{\star}^{\bullet}$ is defined by

$$
\nabla_{\star}^{\bullet_{\star}}:=\left(\mathrm{id} \otimes_{\star} \wedge_{\star}\right) \circ\left(\nabla_{\star} \otimes_{\star} \text { id }\right)+\pi_{\star} \circ\left(\mathrm{id} \otimes_{\star} \mathrm{d}\right),
$$

where $\pi_{\star}: V_{\star} \otimes_{\star} \Omega_{\star}^{\bullet} \rightarrow V_{\star} \otimes_{A_{\star}} \Omega_{\star}^{\bullet}$ is the canonical projection, and id $\otimes_{\star} \wedge_{\star}$ : $\left(V_{\star} \otimes_{A_{\star}} \Omega_{\star}^{\bullet}\right) \otimes_{\star} \Omega_{\star}^{\bullet} \rightarrow V_{\star} \otimes_{A_{\star}} \Omega_{\star}^{\bullet}$ is defined by, for all $v \in V_{\star}$ and $\omega, \tilde{\omega} \in \Omega_{\star}^{\bullet}$,

$$
\left(\operatorname{id~} \otimes_{\star} \wedge_{\star}\right)\left(\left(v \otimes_{A_{\star}} \omega\right) \otimes_{\star} \tilde{\omega}\right):=\left(v \otimes_{A_{\star}} \omega\right) \wedge_{\star} \tilde{\omega}:=v \otimes_{A_{\star}} \omega \wedge_{\star} \tilde{\omega} .
$$

The induced map $\nabla_{\star}^{\bullet_{\star}}: V_{\star} \otimes_{A_{\star}} \Omega_{\star}^{\bullet} \rightarrow V_{\star} \otimes_{A_{\star}} \Omega_{\star}^{\bullet}$ reads explicitly, for all $v \in$ $V_{\star}$ and $\omega \in \Omega_{\star}^{\bullet}, \nabla_{\star}^{\bullet *}\left(v \otimes_{A_{\star}} \omega\right)=\left(\nabla_{\star} v\right) \wedge_{\star} \omega+v \otimes_{A_{\star}} \mathrm{d} \omega$. 
Lemma 7.3. Let $H$ be a Hopf algebra with twist $\mathcal{F} \in H \otimes H, A$ be an ${ }^{H} \mathscr{A}$-algebra, $V$ be an ${ }^{H} \mathscr{M}_{A}$-module and $\left(\Omega^{\bullet}, \wedge, \mathrm{d}\right)$ be a left $H$-covariant differential calculus over $A$. For any $\nabla \in \operatorname{Con}_{A}(V)$ we have the commutative diagram

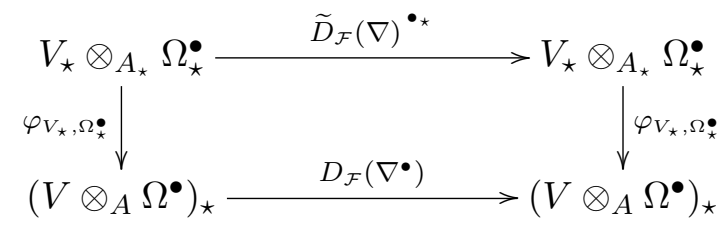

Proof. $H$-equivariance of all maps in (7.1), but $\nabla$, implies

$$
\begin{aligned}
D_{\mathcal{F}}\left(\nabla^{\bullet}\right) & =D_{\mathcal{F}}((\mathrm{id} \otimes \wedge) \circ(\nabla \otimes \mathrm{id})+\pi \circ(\mathrm{id} \otimes \mathrm{d})) \\
& =(\mathrm{id} \otimes \wedge) \circ D_{\mathcal{F}}(\nabla \otimes \mathrm{id})+\pi \circ(\mathrm{id} \otimes \mathrm{d}) .
\end{aligned}
$$

Theorem 5.6 implies

$$
\begin{aligned}
D_{\mathcal{F}}(\nabla \otimes \mathrm{id}) & =\varphi_{V \otimes_{A} \Omega, \Omega} \bullet\left(D_{\mathcal{F}}(\nabla) \otimes_{\star} \mathrm{id}\right) \circ \varphi_{V, \Omega}^{-1} \\
& =\varphi_{V \otimes_{A} \Omega} \cdot, \Omega \circ\left(\varphi_{V_{\star}, \Omega_{\star}} \otimes_{\star} \mathrm{id}\right) \circ\left(\widetilde{D}_{\mathcal{F}}(\nabla) \otimes_{\star} \mathrm{id}\right) \circ \varphi_{V, \Omega}^{-1} \cdot
\end{aligned}
$$

The proof follows from the two commutative diagrams

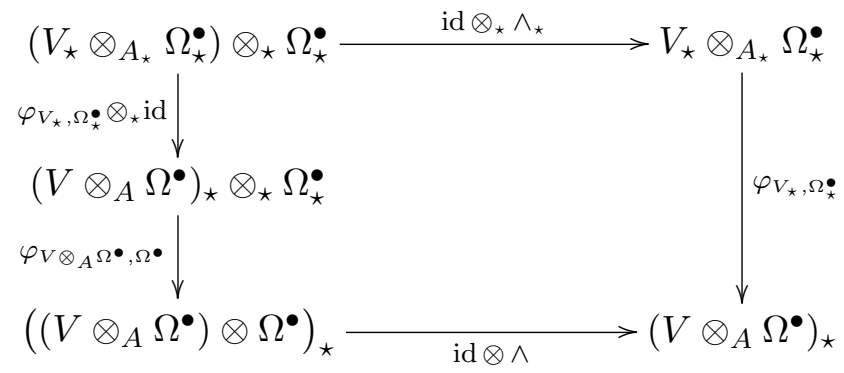

and

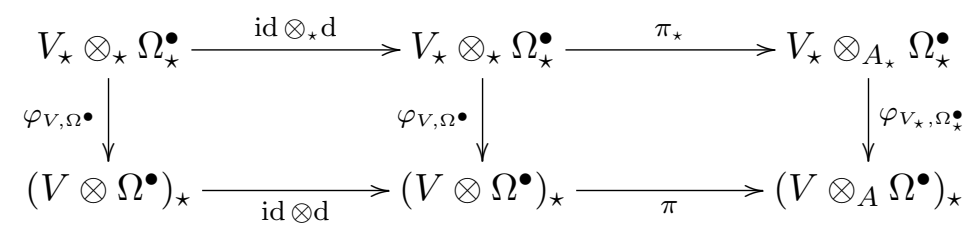

which are a consequence of Theorem 5.6, the $H$-equivariance of the maps and Lemma 5.19. 
The curvature of a connection $\nabla_{\star} \in \operatorname{Con}_{A_{\star}}\left(V_{\star}\right)$ is defined by $R_{\nabla_{\star}}:=$ $\nabla_{\star}^{\bullet_{\star}} \circ \nabla_{\star} \in \operatorname{Hom}_{A_{\star}}\left(V_{\star}, V_{\star} \otimes_{A_{\star}} \Omega_{\star}^{2}\right)$. Due to the isomorphism $\operatorname{Con}_{A}(V) \simeq$ $\operatorname{Con}_{A_{\star}}\left(V_{\star}\right)$ (cf. Theorem 6.5 and the text after the theorem) any connection $\nabla_{\star} \in \operatorname{Con}_{A_{\star}}\left(V_{\star}\right)$ is the image $\widetilde{D}_{\mathcal{F}}(\nabla)$ of a connection $\nabla \in \operatorname{Con}_{A}(V)$.

Theorem 7.4. Let $H$ be a Hopf algebra with twist $\mathcal{F} \in H \otimes H, A$ an ${ }^{H} \mathscr{A}$ algebra, $V$ an ${ }^{H} \mathscr{M}_{A}$-module and $\left(\Omega^{\bullet}, \wedge, \mathrm{d}\right)$ a left $H$-covariant differential calculus over $A$. Consider an arbitrary connection $\widetilde{D}_{\mathcal{F}}(\nabla) \in \operatorname{Con}_{A_{\star}}\left(V_{\star}\right)$, (where $\left.\nabla \in \operatorname{Con}_{A}(V)\right)$. The curvature of the deformed connection $\widetilde{D}_{\mathcal{F}}(\nabla)$ satisfies the identity

$$
R_{\widetilde{D}_{\mathcal{F}}(\nabla)}=\varphi_{V_{\star}, \Omega_{\star}^{2}}^{-1} \circ D_{\mathcal{F}}\left(\nabla^{\bullet} \circ_{\star} \nabla\right)=\widetilde{D}_{\mathcal{F}}\left(\nabla^{\bullet} \circ_{\star} \nabla\right)
$$

Proof. The proof follows from Lemma 7.3 and the property $D_{\mathcal{F}}\left(Q \circ_{\star} \check{Q}\right)=$ $D_{\mathcal{F}}(Q) \circ D_{\mathcal{F}}(\check{Q})$, which holds for any two composable $\mathbb{K}$-linear maps $Q, \check{Q}$.

Notice that the deformed curvature $\widetilde{D}_{\mathcal{F}}\left(R_{\nabla}\right)=\widetilde{D}_{\mathcal{F}}\left(\nabla^{\bullet} \circ \nabla\right)$ differs from the curvature of the deformed connection $R_{\widetilde{D}_{\mathcal{F}}(\nabla)}=\widetilde{D}_{\mathcal{F}}\left(\nabla^{\bullet} \circ_{\star} \nabla\right)$. The right hand side of this latter equality can be recognized as the deformation of the curvature of the connection $\nabla$ seen as an element of the affine space $\operatorname{Con}_{A}(V)_{\star}$ (rather than $\left.\operatorname{Con}_{A}(V)\right)$. Indeed, in this case $\nabla \in \operatorname{Con}_{A}(V)_{\star}$ can be seen as a morphism in the category rep $_{\star}^{H}$, which is characterized by the $\star$-composition $o_{\star}$.

The difference between $\widetilde{D}_{\mathcal{F}}\left(R_{\nabla}\right)$ and $R_{\widetilde{D}_{\mathcal{F}}(\nabla)}$ immediately leads to the following corollary on flat connections.

Corollary 7.5. In the hypotheses of Theorem 7.4, consider an arbitrary connection $\widetilde{D}_{\mathcal{F}}(\nabla) \in \operatorname{Con}_{A_{\star}}\left(V_{\star}\right)$, (where $\nabla \in \operatorname{Con}_{A}(V)$ ). Then the connection $\widetilde{D}_{\mathcal{F}}(\nabla)$ is flat (i.e. $R_{\widetilde{D}_{\mathcal{F}}(\nabla)}=0$ ) if and only if $\nabla^{\bullet} \circ_{\star} \nabla=0$. In general, the deformation of a flat connection $\nabla \in \operatorname{Con}_{A}(V)$ (i.e. $R_{\nabla}=0$ ) is not flat (i.e. $\left.R_{\widetilde{D}_{\mathcal{F}}(\nabla)} \neq 0\right)$. Notice, however, that in the case the flat connection $\nabla \in \operatorname{Con}_{A}(V)$ is additionally $H$-equivariant, then the deformed connection $\widetilde{D}_{\mathcal{F}}(\nabla)$ is also flat.

The study of the cohomology of flat deformed connections could lead to new cohomology invariants or interesting combinations of undeformed ones. 


\subsection{Curvature of the sum of connections}

We conclude this section by calculating the curvature of the sum of two connections. Let $(H, \mathcal{R})$ be a quasitriangular Hopf algebra, $A$ be a quasicommutative ${ }^{H} \mathscr{A}$-algebra, $W$ be a quasi-commutative ${ }^{H}{ }_{A} \mathscr{M}_{A}$-module and $\left(\Omega^{\bullet}, \wedge, \mathrm{d}\right)$ be a graded quasi-commutative left $H$-covariant differential calculus over $A$. For an arbitrary ${ }^{H} \mathscr{M}_{A}$-module $V$ and arbitrary connections $\nabla_{V} \in$ $\operatorname{Con}_{A}(V), \nabla_{W} \in \operatorname{Con}_{A}(W)$, the sum of connections $\nabla_{V} \oplus_{\mathcal{R}} \nabla_{W} \in \operatorname{Con}_{A}(V$ $\otimes_{A} W$ ) is given by (cf. Theorem 6.9), for all $v \in V$ and $w \in W$,

$$
\begin{aligned}
& \left(\nabla_{V} \oplus_{\mathcal{R}} \nabla_{W}\right)\left(v \otimes_{A} w\right) \\
& \quad=\tau_{\mathcal{R} 23}^{-1}\left(\nabla_{V}(v) \otimes_{A} w\right)+\left(\bar{R}^{\beta} \triangleright v\right) \otimes_{A}\left(\bar{R}_{\beta} \triangleright \nabla_{W}\right)(w)
\end{aligned}
$$

We use the following formal notation

$$
\nabla_{V} \oplus_{\mathcal{R}} \nabla_{W}=\tau_{\mathcal{R} 23}^{-1} \circ\left(\nabla_{V} \otimes_{\mathcal{R}} \operatorname{id}_{W}\right)+\operatorname{id}_{V} \otimes_{\mathcal{R}} \nabla_{W},
$$

which is understood to give (7.16) when acting on generating elements $v \otimes_{A}$ $w$. Here all maps are considered to act on the tensor product over $A$ (this is why there are no projections in this expression), and the notation is formal in the sense that the individual terms in (7.17) are not well-defined maps on $V \otimes_{A} W$, but only their sum is. The reason for introducing this compact notation is that it simplifies the lengthy calculation in Proposition 7.6. The equations appearing in the proof of this proposition should be understood as holding true when acting on generating elements $v \otimes_{A} w$ of $V \otimes_{A} W$.

The canonical extension (7.3) of the connection $\nabla_{V} \oplus_{\mathcal{R}} \nabla_{W}$ reads, for all $v \in V, w \in W$ and $\omega \in \Omega^{\bullet}$,

$$
\begin{aligned}
\left(\nabla_{V} \oplus_{\mathcal{R}} \nabla_{W}\right)^{\bullet} & \left(v \otimes_{A} w \otimes_{A} \omega\right) \\
& =\left(\left(\nabla_{V} \oplus_{\mathcal{R}} \nabla_{W}\right)\left(v \otimes_{A} w\right)\right) \wedge \omega+v \otimes_{A} w \otimes_{A} \mathrm{~d} \omega
\end{aligned}
$$

Also here we use a formal notation similar to (7.17),

$$
\begin{aligned}
& \left(\nabla_{V} \oplus_{\mathcal{R}} \nabla_{W}\right)^{\bullet} \\
& \quad=\left(\operatorname{id}_{V \otimes_{A} W} \otimes_{\mathcal{R}} \wedge\right) \circ\left(\left(\nabla_{V} \oplus_{\mathcal{R}} \nabla_{W}\right) \otimes_{\mathcal{R}} \operatorname{id}_{\Omega} \cdot\right)+\operatorname{id}_{V \otimes_{A} W} \otimes_{\mathcal{R}} \mathrm{d} .
\end{aligned}
$$

Again, the individual terms in this expression are not well-defined maps on $V \otimes_{A} W \otimes_{A} \Omega^{\bullet}$, but their sum is.

We express the curvature $R_{\nabla_{V} \oplus_{\mathcal{R}} \nabla_{W}}$ in terms of the curvatures $R_{\nabla_{V}}$ and $R_{\nabla_{W}}$. 
Proposition 7.6. Let $(H, \mathcal{R})$ be a quasitriangular Hopf algebra, $A$ be a quasi-commutative ${ }^{H} \mathscr{A}$-algebra, $W$ be a quasi-commutative ${ }^{{ }_{A}} \mathscr{M}_{A}$-module and $\left(\Omega^{\bullet}, \wedge, \mathrm{d}\right)$ be a graded quasi-commutative left $H$-covariant differential calculus over $A$. Then for any ${ }^{H} \mathscr{M}_{A}$-module $V$ and arbitrary connections $\nabla_{V} \in \operatorname{Con}_{A}(V), \nabla_{W} \in \operatorname{Con}_{A}(W)$, the curvature $R_{\nabla_{V} \oplus_{\mathcal{R}} \nabla_{W}} \in \operatorname{Hom}_{A}\left(V \otimes_{A}\right.$ $\left.W, V \otimes_{A} W \otimes_{A} \Omega^{2}\right)$ satisfies the identity

$$
\begin{aligned}
R_{\nabla_{V} \oplus_{\mathcal{R}} \nabla_{W}}=\tau_{\mathcal{R} 23}^{-1} \circ\left(R_{\nabla_{V}} \otimes_{\mathcal{R}} \operatorname{id}_{W}\right)+\operatorname{id}_{V} \otimes_{\mathcal{R}} R_{\nabla_{W}} \\
+\left(\operatorname{id}_{V \otimes_{A} W} \otimes_{\mathcal{R}} \wedge\right) \circ \tau_{\mathcal{R} 23}^{-1} \circ\left(\nabla_{V} \otimes_{\mathcal{R}} \nabla_{W}\right. \\
\left.-\left(\bar{R}^{\alpha} \triangleright \nabla_{V}\right) \otimes_{\mathcal{R}}\left(\bar{R}_{\alpha} \triangleright \nabla_{W}\right)\right)
\end{aligned}
$$

where $R_{\nabla_{V}} \in \operatorname{Hom}_{A}\left(V, V \otimes_{A} \Omega^{2}\right)$ and $R_{\nabla_{W}} \in \operatorname{Hom}_{A}\left(W, W \otimes_{A} \Omega^{2}\right)$ are the curvatures of $\nabla_{V}$ and $\nabla_{W}$, respectively. The second line in (7.20) is understood in the same formal notation as used in (7.17) and (7.19).

Proof. We shall calculate $R_{\nabla_{V} \oplus_{\mathcal{R}} \nabla_{W}}=\left(\nabla_{V} \oplus_{\mathcal{R}} \nabla_{W}\right) \circ\left(\nabla_{V} \oplus_{\mathcal{R}} \nabla_{W}\right)$. We use $\operatorname{id}_{V \otimes_{A} W}=\operatorname{id}_{V} \otimes_{\mathcal{R}} \operatorname{id}_{W}=\operatorname{id}_{V} \otimes \mathrm{id}_{W}$. In order to simplify the notation we drop all module indices on the identity maps and write $\operatorname{id}_{V \otimes_{A} W} \otimes \wedge=$ id $\otimes$ id $\otimes \wedge=\wedge_{34}$. We also recall that $P \otimes_{\mathcal{R}} Q=P \otimes Q$, whenever the map $Q$ is $H$-equivariant.

Using the notation introduced in (7.17) and (7.19), the curvature is given by the sum of the following four terms

$R_{\nabla_{V} \oplus_{\mathcal{R}} \nabla_{W}}=$

$$
\begin{aligned}
& \left(\wedge_{34} \circ \tau_{\mathcal{R} 23}^{-1} \circ\left(\nabla_{V} \otimes \mathrm{id} \otimes \mathrm{id}\right)+\mathrm{id} \otimes \mathrm{id} \otimes \mathrm{d}\right) \circ \tau_{\mathcal{R} 23}^{-1} \circ\left(\nabla_{V} \otimes \mathrm{id}\right) \\
& +\left(\wedge_{34} \circ\left(\mathrm{id} \otimes_{\mathcal{R}} \nabla_{W} \otimes_{\mathcal{R}} \mathrm{id}\right)+\mathrm{id} \otimes \mathrm{id} \otimes \mathrm{d}\right) \circ\left(\mathrm{id} \otimes_{\mathcal{R}} \nabla_{W}\right) \\
& +\wedge_{34} \circ \tau_{\mathcal{R} 23}^{-1} \circ\left(\nabla_{V} \otimes \mathrm{id} \otimes \mathrm{id}\right) \circ\left(\mathrm{id} \otimes_{\mathcal{R}} \nabla_{W}\right) \\
& +\wedge_{34} \circ\left(\mathrm{id} \otimes_{\mathcal{R}} \nabla_{W} \otimes_{\mathcal{R}} \text { id }\right) \circ \tau_{\mathcal{R} 23}^{-1} \circ\left(\nabla_{V} \otimes \mathrm{id}\right) .
\end{aligned}
$$

We now simplify the individual terms in (7.21). For the first term we find 


$$
\begin{aligned}
& (7.21 \mathrm{a})=\left(\wedge_{34} \circ \tau_{\mathcal{R} 23}^{-1} \circ\left(\nabla_{V} \otimes \mathrm{id} \otimes \mathrm{id}\right) \circ \tau_{\mathcal{R} 23}^{-1}+(\mathrm{id} \otimes \mathrm{id} \otimes \mathrm{d}) \circ \tau_{\mathcal{R} 23}^{-1}\right) \\
& \circ\left(\nabla_{V} \otimes \mathrm{id}\right) \\
& =\left(\wedge_{34} \circ \tau_{\mathcal{R} 23}^{-1} \circ \tau_{\mathcal{R} 34}^{-1} \circ\left(\nabla_{V} \otimes \mathrm{id} \otimes \mathrm{id}\right)+\tau_{\mathcal{R} 23}^{-1} \circ(\mathrm{id} \otimes \mathrm{d} \otimes \mathrm{id})\right) \\
& \circ\left(\nabla_{V} \otimes \mathrm{id}\right) \\
& =\left(\wedge_{34} \circ \tau_{\mathcal{R}(23) 4}^{-1} \circ\left(\nabla_{V} \otimes \mathrm{id} \otimes \mathrm{id}\right)+\tau_{\mathcal{R} 23}^{-1} \circ(\mathrm{id} \otimes \mathrm{d} \otimes \mathrm{id})\right) \\
& \circ\left(\nabla_{V} \otimes \mathrm{id}\right) \\
& =\tau_{\mathcal{R} 23}^{-1} \circ\left(\wedge_{23} \circ\left(\nabla_{V} \otimes \mathrm{id} \otimes \mathrm{id}\right)+\mathrm{id} \otimes \mathrm{d} \otimes \mathrm{id}\right) \circ\left(\nabla_{V} \otimes \mathrm{id}\right) \\
& =\tau_{\mathcal{R} 23}^{-1} \circ\left(\left(\left(\wedge_{23} \circ\left(\nabla_{V} \otimes \mathrm{id}\right)+\mathrm{id} \otimes \mathrm{d}\right) \circ \nabla_{V}\right) \otimes \mathrm{id}\right) \\
& =\tau_{\mathcal{R} 23}^{-1} \circ\left(R_{\nabla_{V}} \otimes \mathrm{id}\right) \text {. }
\end{aligned}
$$

For the second term we find

$$
\begin{aligned}
(7.21 \mathrm{~b}) & =\left(\mathrm{id} \otimes_{\mathcal{R}}\left(\wedge_{23} \circ\left(\nabla_{W} \otimes \mathrm{id}\right)+\mathrm{id} \otimes \mathrm{d}\right)\right) \circ\left(\mathrm{id} \otimes_{\mathcal{R}} \nabla_{W}\right) \\
& =\mathrm{id} \otimes_{\mathcal{R}}\left(\left(\wedge_{23} \circ\left(\nabla_{W} \otimes \mathrm{id}\right)+\mathrm{id} \otimes \mathrm{d}\right) \circ \nabla_{W}\right) \\
& =\mathrm{id} \otimes_{\mathcal{R}} R_{\nabla_{W}} .
\end{aligned}
$$

The third term simplifies to

$$
\begin{aligned}
(7.21 \mathrm{c}) & =\wedge_{34} \circ \tau_{\mathcal{R} 23}^{-1} \circ\left(\nabla_{V} \otimes_{\mathcal{R}}(\mathrm{id} \otimes \mathrm{id})\right) \circ\left(\mathrm{id} \otimes_{\mathcal{R}} \nabla_{W}\right) \\
& =\wedge_{34} \circ \tau_{\mathcal{R} 23}^{-1} \circ\left(\nabla_{V} \otimes_{\mathcal{R}} \nabla_{W}\right)
\end{aligned}
$$

and the last term to

$$
\begin{aligned}
(7.21 \mathrm{~d}) & =\wedge_{34} \circ\left(\mathrm{id} \otimes_{\mathcal{R}}\left(\left(\nabla_{W} \otimes \mathrm{id}\right) \circ \tau_{\mathcal{R}}^{-1}\right)\right) \circ\left(\nabla_{V} \otimes \mathrm{id}\right) \\
& =\wedge_{34} \circ\left(\mathrm{id} \otimes_{\mathcal{R}}\left(\tau_{\mathcal{R} 1(23)}^{-1} \circ\left(\mathrm{id} \otimes_{\mathcal{R}} \nabla_{W}\right)\right)\right) \circ\left(\nabla_{V} \otimes \mathrm{id}\right) \\
& =\wedge_{34} \circ \tau_{\mathcal{R} 2(34)}^{-1} \circ\left(\mathrm{id} \otimes_{\mathcal{R}} \mathrm{id} \otimes_{\mathcal{R}} \nabla_{W}\right) \circ\left(\nabla_{V} \otimes \mathrm{id}\right) \\
& =\wedge_{34} \circ \tau_{\mathcal{R} 2(34)}^{-1} \circ\left(\left(\bar{R}^{\alpha} \triangleright \nabla_{V}\right) \otimes_{\mathcal{R}}\left(\bar{R}_{\alpha} \triangleright \nabla_{W}\right)\right) \\
& =\wedge_{34} \circ \tau_{\mathcal{R} 34}^{-1} \circ \tau_{\mathcal{R} 23}^{-1} \circ\left(\left(\bar{R}^{\alpha} \triangleright \nabla_{V}\right) \otimes_{\mathcal{R}}\left(\bar{R}_{\alpha} \triangleright \nabla_{W}\right)\right) \\
& =-\wedge_{34} \circ \tau_{\mathcal{R} 23}^{-1} \circ\left(\left(\bar{R}^{\alpha}-\nabla_{V}\right) \otimes_{\mathcal{R}}\left(\bar{R}_{\alpha}>\nabla_{W}\right)\right) .
\end{aligned}
$$

The sum of these four terms gives (7.20). 
Remark 7.7. The first line in (7.20) is the sum (of the lift to $V \otimes_{A} W$ ) of the curvatures $R_{\nabla_{V}}$ and $R_{\nabla_{W}}$. The curvature $R_{\nabla_{V} \oplus_{\mathcal{R}} \nabla_{W}}$ is not simply the sum of $R_{\nabla_{V}}$ and $R_{\nabla_{W}}$; the second line in (7.20) gives an additional contribution due to the non $H$-equivariance of the connections. In the special case that either $\nabla_{V}$ or $\nabla_{W}$ are $H$-equivariant, the second line in (7.20) vanishes and the curvature $R_{\nabla_{V} \oplus_{\mathcal{R}} \nabla_{W}}$ is simply the sum of the individual curvatures.

\section{Acknowledgements}

We would like to thank Ugo Bruzzo, Tomasz Brzeziński, Leonardo Castellani, Branislav Jurčo, Thorsten Ohl, Christoph F. Uhlemann and Walter van Suijlekom for useful discussions and comments.

The work of P.A. is in part supported by the exchange grant 2646 of the ESF Activity Quantum Geometry and Quantum Gravity, by the Deutsche Forschungsgmeinschaft through the Research Training Group GRK 1147 Theoretical Astrophysics and Particle Physics and by a short term visit grant of CERN TH Division.

The work of A.S. is in part supported by the Deutsche Forschungsgmeinschaft through the Research Training Group GRK 1147 Theoretical Astrophysics and Particle Physics, by the short visit grant 3267 of the ESF Activity Quantum Geometry and Quantum Gravity and by INFN sezione di Torino, gruppo collegato di Alessandria. A.S. is currently supported by a Research Fellowship of Deutsche Forschungsgemeinschaft (DFG).

\section{References}

[1] P. Aschieri, Twisting all the way: From algebras to morphisms and connections, Int. J. Mod. Phys. Conf. Ser., 13, 1 (2012) arXiv:1210.1143 [math.QA].

[2] P. Aschieri, C. Blohmann, M. Dimitrijevic, F. Meyer, P. Schupp and J. Wess, A Gravity theory on noncommutative spaces, Class. Quant. Grav. 22, 3511 (2005) arXiv:hep-th/0504183.

[3] P. Aschieri and F. Bonechi, On the noncommutative geometry of twisted spheres, Lett. Math. Phys. 59, 133 (2002) arXiv:math/0108136 [math.QA].

[4] P. Aschieri and L. Castellani, Bicovariant calculus on twisted ISO(N), quantum Poincare group and quantum Minkowski space, Int. J. Mod. Phys. A 11, 4513 (1996) arXiv:q-alg/9601006. 
[5] P. Aschieri, M. Dimitrijevic, P. Kulish, F. Lizzi and J. Wess, Noncommutative spacetimes: Symmetries in noncommutative geometry and field theory, Springer, Lecture notes in physics, Vol. 774 (2009).

[6] P. Aschieri, M. Dimitrijevic, F. Meyer and J. Wess, Noncommutative geometry and gravity, Class. Quant. Grav. 23, 1883 (2006) arXiv: hep-th/0510059.

[7] E. Beggs and S. Majid, Nonassociative Riemannian geometry by twisting, Journal of Physics: Conference Series 254, 012002 (2010) arXiv: 0912.1553 [math.QA].

[8] P. Bieliavsky and V. Gayral Deformation Quantization for Actions of Kählerian Lie Groups, arXiv:1109.3419 [math.0A]. To app. in Mem. Amer. Math. Soc..

[9] A. Connes, Non-commutative differential geometry, Publ. Math., Inst. Hautes Étud. Sci. 62, 41 (1985).

[10] A. Connes and M. Dubois-Violette, Noncommutative finite-dimensional manifolds. I. Spherical manifolds and related examples, Commun. Math. Phys. 230, 539 (2002) arXiv:math/0107070 [math.QA].

[11] A. Connes and G. Landi, Noncommutative manifolds: The instanton algebra and isospectral deformations, Commun. Math. Phys. 221, 141 (2001) arXiv:math/0011194 [math.QA].

[12] V. G. Drinfeld, On constant quasiclassical solutions of the Yang-Baxter equations, Soviet Math. Dokl. 28, 667 (1983).

[13] V. G. Drinfeld, Hopf algebras and the quantum Yang-Baxter equation, Soviet Math. Dokl. 32, 254 (1985).

[14] V. G. Drinfeld, Quasi Hopf algebras, Alg. Anal. 1:6, 114 (1989) (Leningrad Math. J. 1:6, 1419 (1990)).

[15] M. Dubois-Violette, Lectures on graded differential algebras and noncommutative geometry, in Noncommutative Differential Geometry and Its Application to Physics, Proceedings of the Workshop Shonan, Japan, June 1999, Y. Maeda, H. Moriyoshi et al (eds), Kluwer Academic Publishers 2001, pp. 245-306 arXiv:math/9912017 [math.QA].

[16] M. Dubois-Violette and T. Masson, On the first order operators in bimodules, Lett. Math. Phys. 37, 467 (1996) arXiv:q-alg/9507028. 
[17] L. D. Faddeev, N. Yu. Reshetikhin and L. A. Takhtajan, Quantization of Lie groups and Lie algebras, Algebra i Anal. 1:1, 178 (1989) (Leningrad Math. J. 1:1, 193 (1990)).

[18] G. Fiore, On second quantization on noncommutative spaces with twisted symmetries, J. Phys. A 43, 155401 (2010) arXiv:0811.0773 [hep-th].

[19] A. Giaquinto and J. J. Zhang, Bialgebra actions, twists, and universal deformation formulas, J. Pure Appl. Alg. 128, 133 (1998) arXiv: hep-th/9411140.

[20] D. Gurevich and S. Majid, Braided groups of Hopf algebras obtained by twisting, Pac. J. Math. 162, 27 (1994).

[21] P. Kulish and A. Mudrov, Twisting adjoint module algebras, Lett. Math. Phys. 95, 233 (2011) arXiv:1011.4758 [math.QA].

[22] T. Lam, Lectures on modules and rings, Graduate Texts in Mathematics 189, Springer-Verlag New York Inc. (1999).

[23] J. Madore, An introduction to noncommutative differential geometry and its physical applications, Lond. Math. Soc. Lect. Note Ser. 257, 1 (2000).

[24] S. Majid, Foundations of quantum group theory, Cambridge, UK: Univ. Pr. (1995).

[25] J. Mourad, Linear Connections In Noncommutative Geometry, Class. Quant. Grav. 12, 965 (1995) arXiv: hep-th/9410201.

[26] N. Yu. Reshetikhin, Quasitriangular Hopf algebras and invariants of tangles, Algebra i Anal. 1:2, 169 (1989) (Leningrad Math. J. 1 2, 491 (1990)).

[27] N. Reshetikhin, Multiparameter Quantum Groups And Twisted Quasitriangular Hopf Algebras, Lett. Math. Phys. 20331 (1990).

[28] M. Rieffel, Deformation quantization for actions of $\mathbb{R}^{d}$, Mem. Amer. Math. Soc. 106 (1993).

[29] A. Schenkel, Noncommutative Gravity and Quantum Field Theory on Noncommutative Curved Spacetimes, $\mathrm{PhD}$ thesis, Würzburg University (2011) arXiv:1210.1115 [math-ph].

[30] A. Schenkel, Twist deformations of module homomorphisms and connections, PoS(CORFU2011)056 (2011) arXiv:1210.1142 [math.QA]. 
[31] A. Sitarz, Twists and spectral triples for isospectral deformations, Lett. Math. Phys. 58 (2001) 69-79 arXiv:math/0102074 [math.QA].

[32] L. A. Takhtadzhyan, Lectures on quantum groups, in Nankai Lectures on Mathematical Physics, Mo-Lin-Ge and Bao-Heng Zhao, Editors World Scientific (1989).

[33] J. C. Varilly, Quantum symmetry groups of noncommutative spheres, Commun. Mathematical Physics 221 (2001) 511 arXiv:math/0102065 [math.QA].

[34] J. Wess, Differential calculus and gauge transformations on a deformed space, Gen. Rel. Grav. 39, 1121 (2007) arXiv:hep-th/0607251.

[35] S. L. Woronowicz, Differential calculus on compact matrix pseudogroups (quantum groups), Commun. Math. Phys. 122, 125 (1989).

Dipartimento di Scienze e Innovazione Tecnologica

AND INFN GRUppo COllegato di Alessandria

Università del Piemonte Orientale

Viale T. Michel 11, 15121 Alessandria, Italy

E-mail address: aschieri@to.infn.it

FACHGRUPPE MATHEMATIK

BERGISCHE UNIVERSITÄT WUPPERTAL

Gaussstrasse 20, 42119 Wuppertal, Germany

E-mail address: schenkel@math.uni-wuppertal.de

PRESENT AdDREss:

Department of Mathematics

Heriot-Watt University

Edinburgh EH14 4AS, United Kingdom

E-mail address: as880@hw.ac.uk 